\title{
Data Report for the NRC/PNL Halden Assembly IFA-432
}
C. R. Hann
D. D. Lanning
E. R. Bradley
R. K. Marshall
M. E. Cunningham
R. E. Williford

August 1978

Prepared for

Fuel Behavior Research Branch

Division of Reactor Safety Research

U.S. Nuclear Regulatory Commission under a Related Services Agreement with the U.S. Department of Energy

Contract EY-76-C-06-1830

Pacific Northwest Laboratory

Operated for the U.S. Department of Energy by Battelle Memorial Institute 


\title{
NOTICE
}

This report was prepared as an account of work sponsored by the United States Government. Neither the United States nor the United States Nuciear Regulatory Commission, nor any of their employees, nor any of their contractors, subcontractors, or their employees, makes any warranty, express or implied, or assumes any legal liability or responsibility for the accuracy, completeness or usefulness of any information, apparatus, product or process disclosed, or represents that its use would not infringe privately owned rights.

\author{
PACIFIC NORTHWEST LABORATORY \\ operated by \\ BATTELLE \\ for the \\ UNITED STATES DEPARTMENT OF ENERGY \\ Under Contract EY-76-C-06-1830
}
Printed in the United States of America Available from
National Technical Info-maticn Service United States Department of Commerce 5205 Port Royal Rand Springfield, Virginia 22757

Price: Printed Copy $s$ •; Mierofiche $\$ 3.00$

NiTS

-Pages Seiling Price

$007-025$

$026-050$

$051-075$

076-100

101-125

126-150

151-175

$176-200$

$201-225$

226-250

251-275

$\$ 4.00$

$\$ 4.50$

$\$ 5.25$

$\$ 6.00$

56.50

57.25

$\$ 8.00$

$\$ 9.00$

$\$ 9.25$

$\$ 9.50$

$\$ 10.75$

$276-300 \quad \$ 11.00$ 


\section{8}

DATA REPORT FOR THE NRC/PNL

HALDEN ASSEMBLY IFA-432
C. R. Hann
D. D. Lannina
E. R. Bradley
R. K. Marsha 11
M. E. Cunninaham
R. E. Wil1iford

August 1978

Prepared for

Fue] Behavior Research Branch Division of Reactor Safety Research U.S. Nuclear Regulatory Commission under a Related Services Agreement with the U.S. Department of Energy Contract EY-76-C-06-1830

Pacific Northwest Laboratory Richland, Washington 99352 
"The NRC will make available data tapes and operational computer codes on research programs dealing with postulated accidents in light water reactors. Persons requesting this information must reimburse the NRC contractors for their expenses in preparing copies of the data tapes and the operational computer codes. Requests should be submitted to the Research Support Branch, Office of Nuclear Regulatory Research, Nuclear Regulatory Commission, Washington, DC 20555." 


\section{ACKNOWLEDGEMENTS}

The support of Dr. W. V. Johnston and H. H. Scott of the Fuel Behavior Research Branch is appreciated. J. A. Christensen contributed to the design and was instrumental in coordinating the final assembly and reactor loading, and, with the Halden Project staff, guiding the assembly through a successful irradiation. N. J. Wildung and W. D. Bennett produced the many data plots for this report. 


\section{CONTENTS}

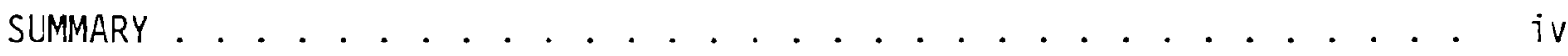
LIST OF FIGURES ..................... . . . .

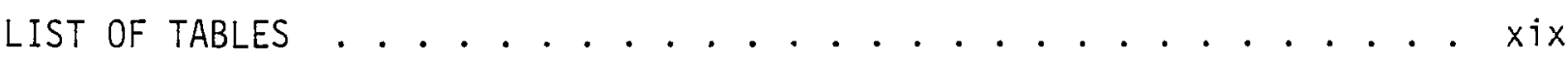

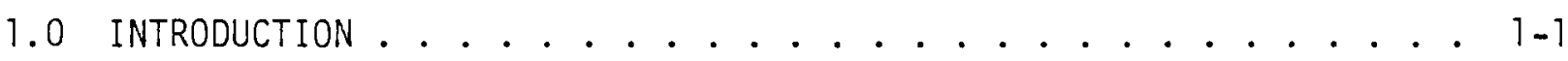

2.0 TEST RATIONALE ....................... 2-1

2.1 Cross-Correlation Efforts . . . . . . . . 2-1

2.2 Test Facility . . . . . . . . . . 2-6

2.3 Fuel Rod Thermal Design Parameters. . . . . . . . 2-6

2.4 Fuel and Cladding Precharacterization . . . . . . 2-11

3.0 DATA PRESENTATION . . . . . . . . . . . . . 3-1

3.1 Power Histories . . . . . . . . . . . . 3-1

3.2 Fuel Temperature Histories. . . . . . . . . . . 3-1

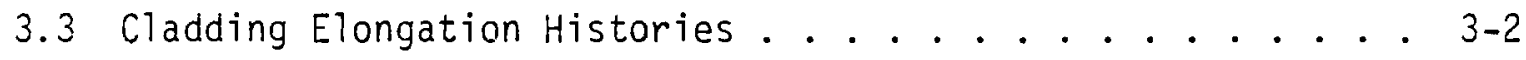

3.4 Rod Internal Pressure Histories ........... 3-2 REFERENCES ................... Ref-1 APPENDIX A - FUEL ROD AND FUEL COLUMN SCHEMATICS FOR IFA-432 . . . . A-1 APPENDIX B - INSTRUMENT DESCRIPTIONS AND CALIBRATION ....... B-1 APPENDIX C - ASSEMBLY POWER CALIBRATION . . . . . . . . . . C C-1 APPENDIX D - DATA PROCESSING .............. D- . . . 


\section{SUMMARY}

This report presents the in-reactor data collected from the NRC/PNL Halden Assembly IFA-432 as a part of the program entitled "Experimental Verification of Steady State Fuel Codes," sponsored by the Fuel Behavior Research Branch of the USNRC. The purpose of this program is to reduce the uncertainties of calculating the thermal stored energy in an operating nuclear fuel rod.

This report presents fuel centerline thermocouple readings, cladding elongation monitor readings, rod internal pressure readings, and neutron detector readings. The neutron detector readings were corrected to represent rod local powers at the thermocouple locations. These data are presented in the form of plots of the variables versus time during the portion of the irradiation period from December 1975 to January 1978. Also included are descriptions of the test rationale, assembly and rod designs, test facility, instrument array and calibration, and data processing methods. Topical reports discussing specific aspects and results of the data analys is are referenced.

As of May 1978, the assembly burnup had reached its design goal of 20,000 MWd/MTM. However, it has been decided to leave the assembly in core to collect high burnup fission gas release data. The xenon-filled Rod 4 was replaced with the non-instrumented Rod 8 (Rod 1 design) after the first cycle. Six of the twelve original thermocouples, four of the six cladding elongation monitors, all SPND'S and all pressure transducers remain operable at this writing. 


\section{FIGURES}

2.1 Arrangement of Temperature Sensors, Neutron Detectors, and Fuel

Relative to Reference Axial Thermal Flux Profile, IFA-431 and 432 2-3

2.2 Schematic of Instrumented Fue? Assembiy--IFA-432 . . . . . . . 2-5

2.3 IFA-431 and IFA-432 Arrangements in the Flow Channel . . . . 2-7

2.4 Schematic of Rod 4 Fuel Column . . . . . . . . . . . . . 2-10

2.5 IFA-432 Fuel Thermal Conductivity 95\% TD Stable, Rods 1, 2, 3, 4 2-12

3.2 Local Linear Heat Ratings at Upper Thermocouple Locations for

Rods 1,3 of IFA 432 - December 1975 . . . . . . . . . . 3-3

3.3 Local Linear Heat Ratings at Lower Thermocouple Locations for

Rods 1,3 of IFA 432 - December 1975 . . . . . . . . . . . 3-3

3.3 Local Linear Heat Ratings at Upper Thermocouple Locations for

Rods 2,4 of IFA 432 - December 1975 . . . . . . . . . . . 3-4

3.4 Local Linear Heat Ratings at Lower Thermocouple Locations for

Rods 2,4 of IFA 432 - December 1975 . . . . . . . . . . . . 3-4

3.5 Local Linear Heat Ratings at Upper Thermocouple Locations for

Rods 5,6 of IFA 432 - December 1975 ............ . . 3-5

3.6 Local Linear Heat Ratings at Lower Thermocouple Locations for

Rods 5,6 of IFA 432 - December 1975 . . . . . . . . . . 3-5

3.7 Local Linear Heat Ratings at Upper Thermocouple Locations for Rods 1,3 of IFA 432 - January 1975 ........... . . . . . .

3.8 Local Linear Heat Ratings at Lower Thermocouple Locations for Rods 1,3 of IFA 432 - January 1975 . . . . . . . . . . 3-6

3.9 Local Linear Heat Ratings at Upper Thermocouple Locations for Rods 2,4 of IFA 432 - January 1975 . . . . . . . . . . . . 3-7

3.10 Local Linear Heat Ratings at Lower Thermocouple Locations for Rods 2,4 of IFA 432 - January 1975 . . . . . . . . . . . 3-7

3.11 Local Linear Heat Ratings at Upper Thermocouple Locations for Rods 5,6 of IFA 432 - January 1975 . . . . . . . . . . 3-8

3.12 Local Linear Heat Ratings at Lower Thermocouple Locations for Rods 5,6 of IFA 432 - January 1975 
3.13 Loca1 Linear Heat Ratings at Upper Thermocouple Locations for Rods 1,3 of IFA 432 - February 1976 .......... 3-9

3.14 Local Linear Heat Ratings at Lower Thermocouple Locations for Rods 1,3 of IFA 432 - February 1976 ........... 3-9

3.15 Local Linear Heat Ratings at Upper Thermocouple Locations for Rods 2,4 of IFA 432 - February 1976 ........... 3-10

3.16 Local Linear Heat Ratings at Lower Thermocouple Locations for Rods 2,4 of IFA 432 - February $1976 \ldots$. . . . . . . . . . 30

3.17 Local Linear Heat Ratings at Upper Thermocouple Locations for Rods 5,6 of IFA 432 - February 1976 ........... 3-11

3.18 Local Linear Heat Ratings at Lower Thermocouple Locations for Rods 5,6 of IFA 432 - February 1976 ............ 3-11

3.19 Local Linear Heat Ratings at Upper Thermocouple Locations for Rods 1,3 of IFA 432 - June 1976 ............ . . 3-12

3.20 Local Linear Heat Ratings at Lower Thermocouple Locations for Rods 1,3 of IFA 432 - June 1976 ............. 3-12

3.21 Local Linear Heat Ratings at Upper Thermocouple Locations for Rods 2,8 of IFA 432 - June $1976 \ldots . . . . . . . .3-13$

3.22 Local Linear Heat Ratings at Lower Thermocouple Locations for Rods 2,8 of IFA 432 - June 1976 .............. 3-13

3.23 Loca1 Linear Heat Ratings at Upper Thermocouple Locations for Rods 5,6 of IFA 432 - June $1976 \ldots . . . . . . . . .3-14$

3.24 Local Linear Heat Ratings at Lower Thermocouple Locations for Rods 5,6 of IFA 432 - June 1976 .............. 3-14

3.25 Local Linear Heat Ratings at Upper Thermocouple Locations for Rods 1,3 of IFA 432 - JuTy 1976 ................ 3-15

3.26 Local Linear Heat Ratings at Lower Thermocouple Locations for Rods 1,3 of IFA 432 - JuTy $1976 \ldots$................ 3-15

3.27 Local Linear Heat Ratings at Upper Thermocouple Locations for Rods 2,8 of IFA 432 - JuTy 1976

3.28 Local Linear Heat Ratings at Lower Thermocouple Locations for Rods 2,8 of IFA 432 - July 1976 
3.29 Local Linear Heat Ratings at Upper Thermocouple Locations for Rods 5,6 of IFA 432 - july $1976 \ldots$. . . . . . . . . . 3-17

3.30 Local Linear Heat Ratings at Lower Thermocouple Locations for Rods 5,6 of IFA 432 - juily $1976 \ldots$. . . . . . . . . . . 37

3.31 Local Linear Heat Ratings at Upper Thermocouple Locations for Rods 1,3 of IFA 432 - August 1976 ............

3.32 Local Linear Heat Ratings at Lower Thermocouple Locations for Rods 1,3 of IFA 432 - August $1976 \ldots 3-18$

3.33 Local Linear Heat Ratings at Upper Thermocouple Locations for

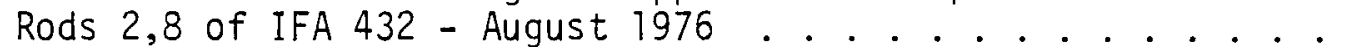

3.34 Local Linear Heat Ratings at Lower Thermocouple Locations for Rods 2,8 of IFA 432 - August 1976 ........... 3-19

3.35 Local Linear Heat Ratings at Upper Thermocouple Locations for Rods 5,6 of IFA 432 - August 1976 ............

3.36 Local Linear Heat Ratings at Lower Thermocouple Locations for Rods 5,6 of IFA 432 - August $1976 \ldots 3-20$

3.37 Local Linear Heat Ratings at Upper Thermocouple Locations for Rods 1,3 of IFA 432 - September 1976

3.38 Local Linear Heat Ratings at Lower Thermocouple Locations for Rods 1,3 of IFA 432 - September 1976 ...........

3.39 Local Linear Heat Ratings at Upper Thermocouple Locations for Rods 2,8 of IFA 432 - September 1976 . . . . . . . . . 3-22

3.40 Local Linear Heat Ratings at Lower Thermocouple Locations for Rods 2,8 of IFA 432 - September $1976 \ldots$. . . . . . . . . 22

3.41 Local Linear Heat Ratings at Upper Thermocouple Locations for Rods 5,6 of IFA 432 - September $197 \overline{6} \ldots . . . . .$.

3.42 Local Linear Heat Ratings at Lower Thermocouple Locations for Rods 5,6 of IFA 432 - September 1976 ..........

3.43 Local Linear Heat Ratings at Upper Thermocouple Locations for Rods 1,3 of IFA 432 - October 1976 .......... 3-24

3.44 Local Linear Heat Ratings at Lower Thermocouple Locations for Rods 1,3 of IFA 432 - October 1976 .......... 3-24

3.45 Local Linear Heat Ratings at Lower Thermocouple Locations for

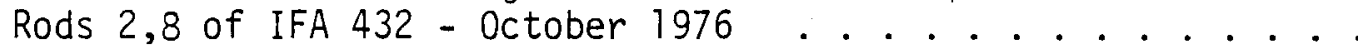


3.46 Local Linear Heat Ratings at Lower Thermocouple Locations for Rods 2,8 of IFA 432 - October 1976 . . . . . . . . . 3-25

3.47 Local Linear Heat Ratings at Upper Thermocouple Locations for Rods 5,6 of IFA 432 - October $1976 \ldots$.............. 3-26

3.48 Local Linear Heat Ratings at Lower Thermocouple Locations for Rods 5,6 of IFA 432 - October $1976 \ldots$. . . . . . . . . . 3-26

3.49 Local Linear Heat Ratings at Lower Thermocouple Locations for Rods 1,3 of IFA 432 - December 1976 .......... 3-27

3.50 Local Linear Heat Ratings at Lower Thermocouple Locations for

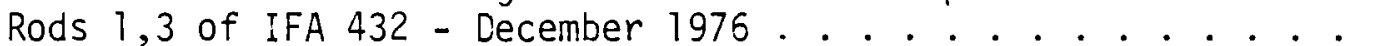

3.51 Local Linear Heat Ratings at Upper Thermocouple Locations for Rods 2,8 of IFA 432 - December 1976 . . . . . . . . . . . . 3-28

3.52 Local Linear Heat Ratings at Lower Thermocouple Locations for Rods 2,8 of IFA 432 - December $1976 \ldots$. . . . . . . . . . 3

3.53 Local Linear Heat Ratings at Upper Thermocouple Locations for Rods 5,6 of IFA 432 - December $1976 \ldots . . . . . .$.

3.54 Local Linear Heat Ratings at Lower Thermocouple Locations for Rods 5,5 of IFA 432 - December 1976 ......... 3-29

3.55 Local Linear Heat Ratings at Upper Thermocouple Locations for Rods 1,3 of IFA 432 - January 1977 ............ 3-30

3.56 Local Linear Heat Ratings at Upper Thermocouple Locations for Rods 1,3 of IFA 432 - January $1977 \ldots$ 3- ............. . . 30

3.57 Local Linear Heat Ratings at Upper Thermocoup?e Locations for Rods 2,8 of IFA 432 - January $1977 \ldots$. . . . . . . . . . 31

3.58 Local Linear Heat Ratings at Lower Thermocouple Locations for Rods 2,8 of IFA 432 - January 1977 ............ 3-31

3.59 Local Linear Heat Ratings at Upper Thermocouple Locations for Rods 5,6 of IFA 432 - January 1977 .......... 3-32

3.60 Local Linear Heat Ratings at Lower Thermocouple Locations for Rods 5,6 of IFA 432 - January $1977 \ldots$ 3-32

3.61 Local Linear Heat Ratings at Upper Thermocouple Locations for Rods 1,3 of IFA 432 - February $1977 \ldots$ 3-33

3.62 Local Linear Heat Ratings at Lower Thermocouple Locations for

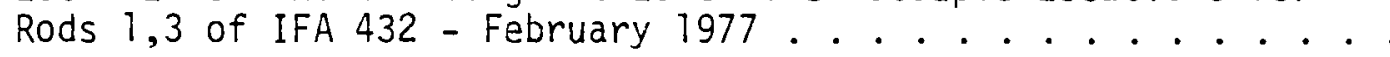


3.63 Local Linear Heat Ratings at Upper Thermocouple Locations for Rods 2,8 of IFA 432 - February 1977 .......... 3-34

3.64 Local Linear Heat Ratings at Lower Thermocouple Locations for Rods 2.8 of IFA 432 - February $1977 \ldots$. . . . . . . . 34

3.65 Local Linear Heat Ratings at Upper Thermocouple Locations for Rods 5,6 of IFA 432 - February $1977 \ldots . . . . . . .3-35$

3.66 Local Linear Heat Ratings at Lower Thermocouple Locations for Rods 5,6 of IFA 432 - February $1977 \ldots \ldots$ 3-35

3.67 Local LInear Heat Ratings at Upper Thermocouple Locations for Rods 1,3 of IFA 432 - March $1977 \ldots$ 3-36

3.68 Local Linear Heat Ratings at Lower Thermocouple Locations for Rods 1,3 of IFA 432 - March $1977 \ldots$. . . . . . . . . 36

3.69 Local Linear Heat Ratings at Upper Thermocouple Locations for Rods 2,8 of IFA 432 - March $1977 \ldots$. . . . . . . . . . 37

3.70 Local Linear Heat Ratings at Lower Thermocouple Locations for Rods 2,8 of IFA 432 - March $1977 \ldots$. . . . . . . . 3-37

3.71 Local Linear Heat Ratings at Upper Thermocouple Locations for Rods 5,6 of IFA 432 - March $1977 \ldots . . . . . . .3-38$

3.72 Local Linear Heat Ratings at Lower Thermocouple Locations for Rods 5,6 of IFA 432 - March 1977 ........... 3-38

3.73 Local Linear Heat Ratings at Upper Thermocouple Locations for Rods 1,3 of IFA 432 - Apri1 1977 ............. 3-39

3.74 Local Linear Heat Ratings at Lower Thermocouple Locations for Rods 1,3 of IFA 432 - Apri1 $1977 \ldots$............. 3-39

3.75 Local Linear Heat Ratings at Upper Thermocouple Locations for Rods 2,8 of IFA 432 - Apri1 1977 ............. 3-40

3.76 Local Linear Heat Ratings at Lower Thermocouple Locations for Rods 2,8 of IFA 432 - April 1977 .......... 3-40

3.77 Local Linear Heat Ratings at Upper Thermocouple Locations for Rods 5,6 of IFA 432 - Aprit $1977 \ldots$ 3-41

3.78 Local Linear Heat Ratings at Lower Thermocouple Locations for Rods 5,6 of IFA 432 - Apri1 $1977 \ldots \ldots$ 3-4

3.79 Local Linear Heat Ratings at Upper Thermocouple Locations for Rods 1,3 of IFA 432 - May $1977 \ldots . . . . . . .3-42$ 
3.80 Local Linear Heat Ratings at Lower Thermocouple Locations for Rods 1,3 of IFA 432 - May 1977 . . . . . . . . . . . 3-42

3.81 Local Linear Heat Ratings at Upper Thermocouple Locations for Rods 2,8 of IFA 432 - May $1977 \ldots$. . . . . . . . . . . 33

3.82 Local Linear Heat Ratings at Lower Thermocouple Locations for Rods 2,8 of IFA 432 - May $1977 \ldots . . . . . . . .3-43$

3.83 Local Linear Heat Ratings at Upper Thermocouple Locations for Rods 5,6 of IFA 432 - May $1977 \ldots . . . . . . .3-44$

3.84 Local Linear Heat Ratings at Lower Thermocouple Locations for Rods 5,6 of IFA 432 - May $1977 \ldots . . . . . . .344$

3.85 Local Linear Heat Ratings at Upper Thermocouple Locations for Rods 1,3 of IFA 432 - June $1977 \ldots . . . . . . . .3-45$

3.86 Local Linear Heat Ratings at Lower Thermocouple Locations for Rods 1,3 of IFA 432 - June 1977 .............. 3-45

3.87 Local Linear Heat Ratings at Upper Thermocouple Locations for Rods 2,8 of IFA 432 - June $1977 \ldots \ldots$ 3-4. . . . . . . 36

3.88 Local Linear Heat Ratings at Lower Thermocouple Locations for Rods 2,8 of IFA 432 - June $1977 \ldots . . . . . . .3-46$

3.89 Local Linear Heat Ratings at Upper Thermocouple Locations for

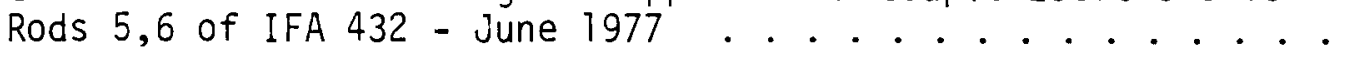

3.90 Local Linear Heat Ratings at Lower Thermocouple Locations for Rods 5,6 of IFA 432 - June $1977 \ldots \ldots$ 3- . . . . . . . 37

3.91 Local Linear Heat Ratings at Upper Thermocouple Locations for Rods 1,3 of IFA 432 - July $1977 \ldots \ldots$ 3-48

3.92 Local Linear Heat Ratings at Lower Thermocouple Locations for Rods 1,3 of IFA 432 - July $1977 \ldots \ldots$. . . . . . . . . 48

3.93 Local Linear Heat Ratings at Upper Thermocouple Locations for Rods 2,8 of IFA 432 - July $1977 \ldots \ldots$ 3-49 . . . . . . . .

3.94 Local Linear Heat Ratings at Lower Thermocouple Locations for Rods 2, 8 of IFA 432 - July $1977 \ldots$. . . . . . . . . 3-49

3.95 Local Linear Heat Ratings at Upper Thermocouple Locations for Rods 5,6 of IFA 432 - July $1977 \ldots$. . . . . . . . . . 50

3.96 Local Linear Heat Ratings at Lower Thermocouple Locations for

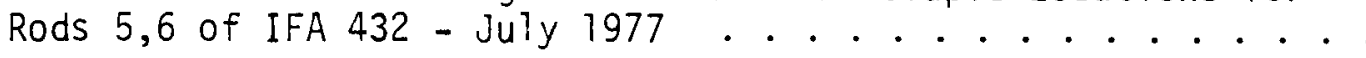


3.97 Local Linear Heat Ratings at Upper Thermocouple Locations for Rods 1,3 of IFA 432 - dugust $1977 \ldots . . . . . . .3-51$

3.98 Local Linear Heat Ratings at Lower Thermocouple Locations for Rods 1,3 of IFA 432 - August 1977.............. 3-51

3.99 Local Linear Heat Ratings at Upper Thermocouple Locations for Rods 2,8 of IFA 432 - August 1977 ............. 3-52

3.100 Local Linear Heat Ratings at Lower Thermocouple Locations for Rods 2,8 of IFA 432 - August $1977 \ldots$ 3-52

3.101 Local Linear Heat Ratings at Upper Thermocouple Locations for Rods 5,6 of IFA 432 - August $1977 \ldots 3-53$

3.102 Local Linear Heat Ratings at Lower Thermocouple Locations for Rods 5,6 of IFA 432 - August $1977 \ldots$. . . . . . . . . 33

3.103 Local Linear Heat Ratings at Upper Thermocouple Locations for Rods 1,3 of IFA 432 - October $1977 \ldots . . . . . .3-54$

3.104 Local Linear Heat Ratings at Lower Thermocouple Locations for Rods 1,3 of IFA 432 - October 1977 ............ 3-54

3.105 Local Linear Heat Ratings at Upper Thermocouple Locations for Rods 2,8 of IFA 432 - October 1977 ............ 3-55

3.106 Local Linear Heat Ratings at Lower Thermocouple Locations for Rods 2,8 of IFA 432 - October 1977 ............. 3-55

3.107 Local Linear Heat Ratings at Upper Thermocouple Locations for Rods 5,6 of IFA 432 - October $1977 \ldots$............ 3-56

3.108 Local Linear Heat Ratings at Lower Thermocouple Locations for Rods 5,6 of IFA 432 - October $1977 \ldots$............. 3-56

3.109 Local Linear Heat Ratings at Upper Thermocouple Locations for Rods 1,3 of IFA 432 - December 1977 . . . . . . . . 3-57

3.110 Local Linear Heat Ratings at Lower Thermocouple Locations for Rods 1,3 of IFA 432 - December 1977 .......... 3-57

3.111 Loca1 Linear Heat Ratings at Upper Thermocouple Locations for Rods 2,8 of IFA 432 - December $1977 \ldots 3-58$

3.112 Local Linear Heat Ratings at Lower Thermocouple Locations for Rods 2,8 of IFA 432 - December 1977 . . . . . . . . 3-58

3.113 Local Linear Heat Ratings at Upper Thermocouple Locations for Rods 5,6 of IFA 432 - December 1977 
3.114 Local Linear Heat Ratings at Lower Thermocouple Locations for

Rods 5,6 of IFA 432 - December $1977 \ldots . . . . . . .3-59$

3.115 Local Linear Heat Ratings at Upper Thermocouple Locations for

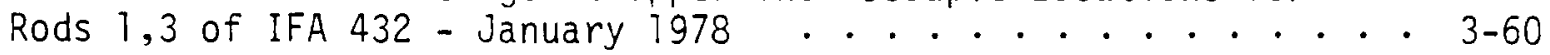

3.116 Local Linear Heat Ratings at Lower Thermocouple Locations for

Rods 1,3 of IFA 432 - January $1978 \ldots$ 3-60

3.117 Local Linear Heat Ratings at Upper Thermocouple Locations for

Rods 2,8 of IFA 432 - January 1978 . . . . . . . . . 3-61

3.118 Local Linear Heat Ratings at Lower Thermocouple Locations for

Rods 2,8 of IFA 432 - January 1978 . . . . . . . 3-61

3.119 Local Linear Heat Ratings at Upper Thermocouple Locations for

Rods 5,6 of IFA 432 - January 7978 . . . . . . . 3-62

3.120 Local Linear Heat Ratings at Lower Thermocouple Locations for

Rods 5,6 of IFA 432 - January $1978 \ldots$. . . . . . . 3-62

3.121 Upper Thermocouple Readings for Rods 1,3 of IFA 432 -

December 1975 .................... 3-63

3.122 Lower Thermocouple Readings for Rods 1,3 of IFA 432 -

December 1975 ................... 3-63

3.123 Upper Thermocouple Readings for Rod 4 of IFA 432 -

December 1975 . . . . . . . . . . . . . . 3-64

3.124 Lower Thermocouple Readings for Rods 2,4 of IFA 432 -

December 1975 . . . . . . . . . . . 3-64

3.125 Upper Thermocouple Readings for Rods 5,6 of IFA 432 -

December 1975 ................... 3-65

3.126 Lower Thermocouple Readings for Rods 5,6 of IFA 432 -

December 1975 . . . . . . . . . . . . . 3-65

3.127 Upper Thermocouple Readings for Rods 1,3 of IFA 432 -

January 1976 ................... 3-66

3.128 Lower Thermocouple Readings for Rods 1,2,3 of IFA 432 -

January 1976.................... . . . . . . . . . . . . . . . .

3.129 Upper Thermocouple Readings for Rods 5,6 of IFA 432 -

January 1976 . . . . . . . . . . . . . . . 3-67

2.130 Lower Thermocouple Readings for Rods 5,6 of IFA 432 -

January 1976 . . . . . . . . . . . . . . . 3-67 
3.131 Upper Thermocouple Readings for Rods 1,3 of IFA 432 February 1976 . . . . . . . . . . . . . 3-68

3.132 Lower Thermocouple Readings for Rods 1,2,3 of IFA 432 -

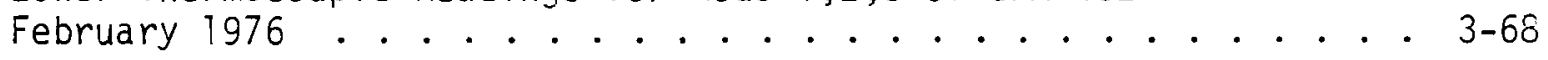

3.133 Upper Thermocouple Readings for Rods 5,6 of IFA 432 -

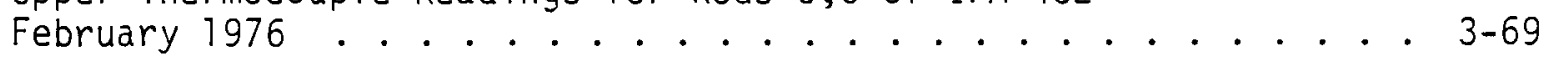

3.134 Lower Thermocouple Readings for Rods 5,6 of IFA 432 -

February 1976 ................ 3-69

3.135 Upper Thermocouple Readings for Rods 1,3 of IFA 432 -

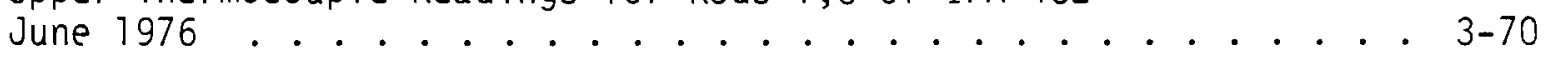

3.136 Lower Thermocouple Readings for Rods $1,2,3$ of IFA 432 -

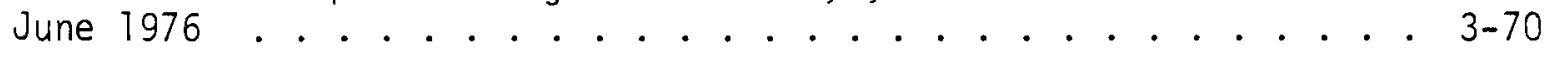

3.137 Upper Thermocouple Readings for Rods 5,6 of IFA 432 June 1976 ..................... 3-71

3.138 Lower Thermocouple Readings for Rods 5,6 of IFA 432 June $1976 \ldots \ldots$. . . . . . . . . . . . . . . . . . . . . . .

3.139 Upper Thermocouple Readings for Rods 1,3,6 of IFA 432 - . . . . 3-72

3.140 Lower Thermocouple Readings for Rods $1,2,3$ of IFA 432 JuTy 1976 . . . . . . . . . . . . . . . . . . . . . . . . . . . . .

3.141 Lower Thermocouple Readings for Rods 5,6 of IFA 432 .
July 1976 . . . . . . . . . . . . . . . . . . . . . . . .

3.142 Upper Thermocouple Readings for Rods 1,3,6 of IFA $432 \ldots$

3.143 Lower Thermocouple Readings for Rods 1,2,3 of IFA 432 August 1976 .................. 3- . . . . . . . . . .

3.144 Lower Thermocouple Readings for Rods 5,6 of IFA 432 August 1976 .................. . . 3-74

3.145 Upper Thermocouple Readings for Rods 1,3,6 of IFA 432 September 1976................. 3- . . . . . . . . . .

3.146 Lower Thermocouple Readings for Rods 1,2,3 of IFA 432 September 1976.................. . 3-75

3.147 Lower Thermocouple Readings for Rods 5,6 of IFA 432 September 1976..................... 3-76 
3.148 Upper Thermecouple Readings for Rods 1,3,6 of IFA 432 October 1976....................... 3-76

3.149 Lower Thermocouple Readings for Rods 1,2,3 of IFA 432 -

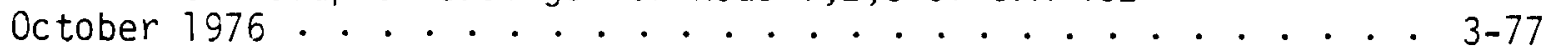

3.150 Lower Thermocouple Readings for Rods 5,6 of IFA 432 -

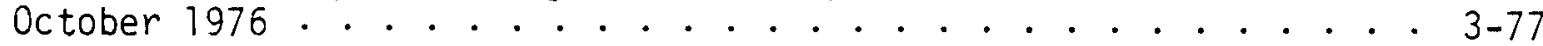

3.151 Upper Thermocouple Readings for Rods 1,3 of IFA 432 -

December 1976 . . . . . . . . . . . . . . . . 3-78

3.152 Lower Thermocouple Readings for Rods 1,2,3 of IFA 432 -

December 1976 . . . . . . . . . . . . . 3-78

3.153 Lower Thermocouple Readings for Rods 5,6 of IFA 432 -

December 1976 . . . . . . . . . . . . . . 3-79

3.154 Upper Thermocouple Readings for Rods 1,3 of IFA 432 -

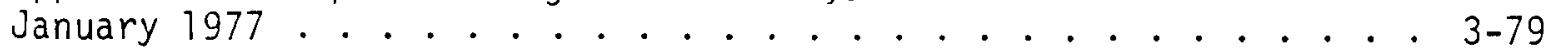

3.155 Lower Thermocouple Readings for Rods 1,2,3 of IFA 432 -

January 1977 .................. . . 3-80

3.156 Lower Thermocouple Readings for Rods 5,6 of IFA 432 -

January 1977 . . . . . . . . . . . . . . . . . 3-80

3.157 Upper Thermocouple Readings for Rod 3 of IFA 432 -

February 1977 . . . . . . . . . . . . . . . 3-81

3.158 Lower Thermocouple Readings for Rods 1,2,3 of IFA 432 -

February 1977 . . . . . . . . . . . . . . . 3-81

3.159 Lower Thermocouple Readings for Rods 5,6 of IFA 432 -

February 1977 ... . . . . . . . . . . 3-82

3.160 Upper Thermocouple Readings for Rod 3 of IFA 432 -

March 1977 .................. . . 3-82

3.161 Lower Thermocouple Readings for Rods 1,2,3 of IFA 432 -

March 1977 . . . . . . . . . . . . . . . . . 3-83

3.162 Lower Thermocouple Readings for Rods 5,6 of IFA 432 -

March 1977 . . . . . . . . . . . . . . . . . 3-83

3.163 Upper Thermocouple Readings for Rod 3 of IFA 432 -

April 1977 .................. . . 3-84

3.164 Lower Thermocouple Readings for Rods 1,2,3 of IFA 432 -

April 1977 . . . . . . . . . . . . . . . 3-84 
3.165 Lower Thermocouple Readings for Rods 5,6 of IFA 432 -

Apri1 1977.................. 3-85

3.166 Upper Thermocouple Readings for Rod 3 of IFA 432 -

May 1977................. . . 3-85

3.167 Lower Thermocouple Readings for Rods 1,2,3 of IFA 432 -

May 1977 . . . . . . . . . . . . . . 3-86

3.168 Lower Thermocouple Readings for Rods 5,6 of IFA 432 -

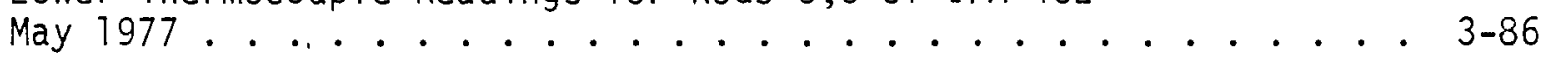

3.169 Upper Thermocouple Readings for Rod 3 of IFA 432 -

June 1977 .................. 3-87

3.170 Lower Thermocouple Readings for Rods 1,2,3 of IFA 432 -

June $1977 \ldots \ldots$................... 3-87

3.171 Lower Thermocouple Readings for Rods 5,6 of IFA 432 -

June $1977 \ldots \ldots$. . . . . . . . . . . . . . . . . . . . .

3.172 Upper Thermocouple Readings for Rod 3 of IFA 432 -

July 1977 . . . . . . . . . . . . . . . . . 3-88

3.173 Lower Thermocouple Readings for Rods 1,2,3 of IFA 432 -

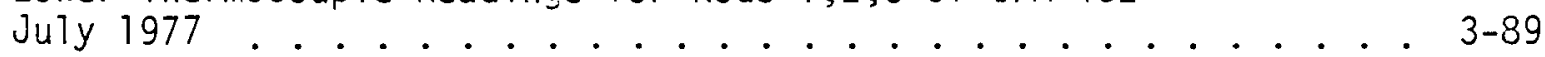

3.174 Lower Thermocouple Readings for Rods 5,6 of IFA 432 -

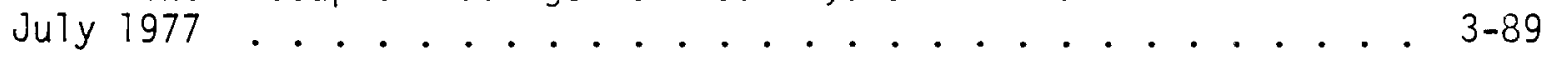

3.175 Upper Thermocouple Readings for Rod 3 of IFA 432 -

August $1977 \ldots \ldots$. . . . . . . . . . . . . . . . . .

3.176 Lower Thermocouple Readings for Rods 1,2,3 of IFA 432 -

August $1977 \ldots \ldots$. . . . . . . . . . . . . . . .

3.177 Lower Thermocouple Readings for Rods 5,6 of IFA 432 -

August 1977

3.178 Upper Thermocouple Readings for Rod 3 of IFA 432 October 1977 .................. 3- . . . . .

3.179 Lower Thermocouple Readings for Rods 1,2,3 of IFA 432 October 1977

3.180 Lower Thermocouple Readings for Rods 5,6 of IFA 432 October 1977

3.181 Upper Thermocouple Readings for Rod 3 Of IFA 432 December 1977 
3.182 Lower Thermocouple Readings for Rods 1,2,3 of IFA 432 -

December 1977 . . . . . . . . . . . . . . 3-93

3.183 Lower Thermocouple Readings for Rods 5,6, of IFA 432 -

December 1977 . . . . . . . . . . . . . . . 3-94

3.184 Upper Thermocouple Readings for Rod 3 or IFA 432 -

January 1978 . . . . . . . . . . . . . . . . . . . . . 3-94

3.185 Lower Thermocouple Readings for Rods 1,2,3 cf IFA 432 -

January 1978 . . . . . . . . . . . . . . . . . 3-95

3.186 Lower Thermocouple Readings for Rods 5,6 of IFA 432 -

January 1978 . . . . . . . . . . . . . . . . . . 3-95

3.187 Cladding Elongation Sensor Readings for Rods $1,2,5,6$ of

IFA 432 - December $1975 \ldots . . . . . . . .3-96$

3.188 Cladding Elongation Sensor Readings for Rods 3,4 of

IFA 432 - December 1975 . . . . . . . . . . . . 3-96

3.189 Cladding Elongation Sensor Readings for Rods $1,2,5,6$ of

IFA 432 - January $1976 \ldots$. . . . . . . . . . . . . . . . . . .

3.190 Cladding Elongation Sensor Readings for Rods 3,4 of

IFA 432 - January $1976 \ldots$. . . . . . . . . . . . . . . . . . .

3.191 Cladding Elongation Sensor Readings for Rods 1,2,5,6 of

IFA 432 - February $1976 \ldots . . . . . . . .3-98$

3.192 Cladding Elongation Sensor Readings for Rods 3,4 of

IFA 432 - February $1976 \ldots$............. 3-98

3.193 Cladding Elongation Sensor Readings for Rods 2,3,6,8 of

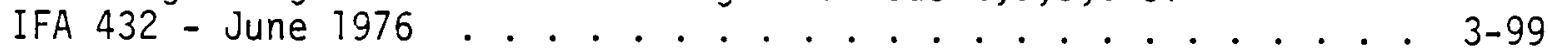

3.194 Cladding Elongation Sensor Readings for Rods 2,3,6,8 of

IFA 432 - July $1976 \ldots . . . . . . . . . . . .3-99$

3.195 Cladding Elongation Sensor Readings for Rods 2,3,6,8 of

IFA 432 - August $1976 \ldots$.................... 3-100

3.196 Cladding Elongation Sensor Readings for Rods 2,3,6,8 of

IFA 432 - September $1976 \ldots$. . . . . . . . . . . . . . . . . . . .

3.197 Cladding Elongation Sensor Readings for Rods 2,3,6,8 of

IFA 432 - October 1976 . . . . . . . . . . . 3-101

3.198 Cladding Elongation Sensor Readings for Rods 2,3,6,8 of IFA 432 - December 1976 
3.199 Cladding Elongation Sensor Readings for Rods 2,3,6,8 of IFA 432 - January 1977 . . . . . . . . . . . . . . . . . . .

3.200 Cladding Elongation Sensor Readings for Rods 2,3,6,8 of IFA 432 - February $1977 \ldots$. . . . . . . . . . . . . . . .

3.201 Cladding Elongation Sensor Readings for Rods 2,3,6,8 of IFA 432 - March 1977

3.202 Cladding Elongation Sensor Readings for Rods 2,3,6,8 of IFA 432 - Apri1 1977 .................. 3-103

3.203 Cladding Elongation Sensor Readings for Rods 2,3,6,8 of IFA 432 - May 1977 . . . . . . . . . . . . . . 3-104

3.204 Cladding Elongation Sensor Readings for Rods 2,3,6,8 of

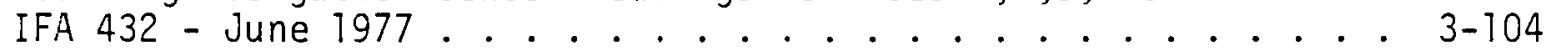

3.205 Cladding Elongation Sensor Readings for Rods 2,3,6,8 of

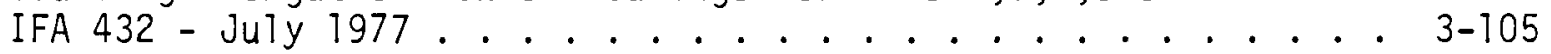

3.206 Cladding Elongation Sensor Readings for Rods 2,3,6,8 of IFA 432 - August $1977 \ldots$. . . . . . . . . . . . . . .

3.207 Cladding Elongation Sensor Readings for Rods 2,3,6,8 of IFA 432 - October $1977 \ldots \ldots$. . . . . . . . . . . . . . . . .

3.208 Cladding Elongation Sensor Readings for Rods 2,3,6,8 of

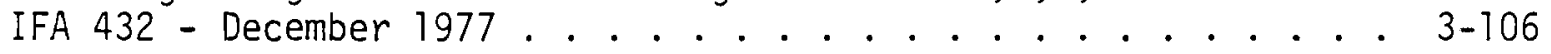

3.209 Cladding Elongation Sensor Readings for Rods 2,3,6,8 of IFA 432 - January 1978 ............. 3-107

3.210 Internal Pressures for Rods 1,5,6 of IFA 432 - December 1975 . 3-107

3.211 Internal Pressures for Rods 1,5,6 of IFA 432 - January 1976 . . 3-108

3.212 Internal Pressures for Rods 1,5,6 of IFA 432 - February 1976 . . 3-108

3.213 Internal Pressures for Rods 1,5,6 of IFA 432 - June 1976 . . . 3-109

3.214 Internal Pressures for Rods 1,5,6 of IFA 432 - August 1976 . . 3-109

3.215 Internal Pressures for Rods 1,5,6 of IFA 432 - September 1976 . 3-110

3.216 Internal Pressures for Rods 1,5,6 of IFA 432 - October 1976 . . 3-110

3.217 Internal Pressures for Rods 1,5,6 of IFA 432 - December 1976 . 3-111

3.218 Internal Pressures for Rods 1,5,6 of IFA 432 - January 1977 . . 3-111 
3.219 Internal Pressures for Rods 1,5,6 of IFA 432 - February 1977 . . 3-112

3.220 Internal Pressures for Rods 1,5,6 of IFA 432 - March 1977 . . 3-112

3.221 Internal Pressures for Rods 1,5,6 of IFA 432 - Apri1 1977 . . 3-113

3.222 Internal Pressures for Rods 1,5,6 of IFA 432 - May 1977 . . . 3-113

3.223 Internal Pressures for Rods 1,5,6 of IFA 432 - June 1977 . . . 3-114

3.224 Internal Pressures for Rods 1,5,6 of IFA 432 - July 1977 . . . 3-114

3.225 Internal Pressures for Rods 1,5,6 of IFA 432 - August 1977 . . 3-115

3.226 Internal Pressures for Rods 1,5,6 of IFA 432 - October 1977 . . 3-115

A-1 Schematic Arrangement of Fuel Rods for IFA $432 \ldots . . . .$. A-1

A-2 Stack Arrangement for Rods 1 and $6 \ldots \ldots$ A 2

A-3 Stack Arrangement for Rods 2, 3, and 5 ......... A-3

A-4 Stack Arrangement for Rod 2 - IFA 432 . . . . . . . . . A A-4

A-5 Stack Arrangement for Rod $4 \ldots \ldots$. . . . . . A-5

A-6 Stack Arrangement for Non-Istrumented Rods 7, 8, 9 - IFA 432 . A-6

B-1 Schematic of Self-Powered, Beta Current Neutron Detector. . . . B-2

B-2 Schematic of W $5 \%$ Re/W $26 \%$ Re Thermocouples with

Grounded Junction . . . . . . . . . . . . . . . . B-4

B-3 Calibration Curve for $\mathrm{W} 5 \% \operatorname{Re} / \mathrm{W} 26 \%$ Re Thermocouples...... B-4

B-4 Cladding Elongation Monitor . . . . . . . . . . . B-6

B-5 Fission Gas Pressure Transducer . . . . . . . . . . . B-8

D-1 Flow Diagram for Processing Halden Data ........ D-2

D-2 Flow Diagram for Correction of Halden Rod Local Powers .... D-3

D-3 Typical Axial Power Profile for IFA $432 \ldots \ldots$. . . . . . D

D-4 Flow Diagram for Gap Conductance and Uncertainty Calculations . D-6 


\section{TABLES}

2.1 Design Parameters and Instrumentation for IFA-43? and IFA-432 . . 2-2

2.2 Cross-Correlation Matrix . . . . . . . . . . . . . . 2-6

2.3 Operating Data for the HBWR . . . . . . . . . . . . . . . 2-6

3.1 IFA-432 Burnup .................... . . 3-116

B. 1 IFA-432 Coolant Temperatures . . . . . . . . . . . . . . B-9

B.2 Instrument Failure Dates for IFA-432 . . . . . . . . . . B-10

0.1 Typical Power Correction Factors for IFA-432 . . . . . . . . . D-2 
. 


\subsection{INTRODUCTION}

The thermal stored energy in a fuel rod is the driving function for the severest postulated nuclear energy-related accident, the loss-of-coolantaccident (LOCA). Because of this, the final acceptance criteria for emergency core cooling systems requires calculation of the stored energy and gap conductance of a fuel rod, both for normal operation and for the duration of the LOCA. Although these calculations are used in the regulation of commercial nuclear power plants, uncertainties in them have caused temporary derating of many power plants and delays in the startup of additional plants. Many of these uncertainties can be attributed to the lack of well-characterized data for fuel irradiated throughout the normal operating power range of commercial nuclear power plants.

Due to this lack of wel1-characterized data, some computer-coded mathematical models that have been developed to simulate fuel rod behavior over a wide range of postulated conditions are inadequate. These factors together contribute substantially to the stored energy uncertainty. Specifically, the effects of fuel densification and fission gas on the fuel-cladding gap conductance are difficult to quantify, primarily because the applicable data are extremely sparse. As a result, although the total conductance of the fuel rod must be considered in stored energy calculations, the largest uncertainties in those calculations are associated with changes in the gap conditions and their concomitant effects on the gap conductance.

To focus on these uncertainties, two instrumented fuel assemblies have been designed and are being irradiated in the Halden Boiling Water Reactor (HBWR). The fuel assemblies have been designated: IFA-431 - this assembly had a design power of $10 \mathrm{~kW} / \mathrm{ft}(328 \mathrm{~W} / \mathrm{cm})$ and reached its goal burnup of 4000 MWd/MTM in February 1976; IFA-432 - this assembly has a design power of $15 \mathrm{~kW} / \mathrm{ft}(492 \mathrm{~W} / \mathrm{cm})$ and reached its goal burnup of $20,000 \mathrm{MWd} / \mathrm{MTM}$ in late1978. However, since most of the instruments are still functioning properly 
(See Appendix B), it was decided to leave IFA-432 in the Halden core to obtain much-needed data at higher burnups.

These instrumented fuel assemblies are providing well-characterized experimental data under conditions that realistically simulate light water reactor (LWR) operations. The data will be used to verify United States Nuclear Regulatory Commission (NRC) fuel codes, and it will provide benchmark data for commercial fuel licensing codes submitted by nuclear fuel vendors. 


\subsection{TEST RATIONALE}

Experimental verification of computer codes provides a means to quantify uncertainties in simulating the conditions for an operating nuclear fuei rod. A collection of mathematical models (i.e., a computer code) is used to simulate the wide range of conditions postulated curing an evaluation of reactor fuel safety. Any computer code that is forced to rely on a collection of empirical and semiempirical models for much of the analys is is limited and should be primarily used for interpolation. Some extrapolation can be accomplished with models based on first principles; however, well-characterized data are needed in either case to test code predictions. When these tests were designed (July 1974), very little data were available describing the effects of burnup on LWR fuel and no data were available describing the effects of fuei densification on fuel temperatures. Accordingly, a test matrix was developed (Table 2.1), and two instrumented assemblies were designed to provide the data.

\subsection{CROSS-CORRELATION EFFORTS}

Much thought went into the design of the tests to ensure a means for cross correlating the data, provide as many independent checks of data validity as possible, ensure against instrument failure, ensure at least internal consistency on a relative basis, and provide some reference points to commercial plant designs as well as other fuel research programs.

One of the test design basic premises was to provide a systematic approach that would allow adequate interpolation and extrapolation with computer codes. The first step in this approach was the decision to design IFA-431 and IFA-432 as identical assemblies. An identical design enhances the ability to interpolate over a range of powers and also replicates initial conditions. For example, a11 the data from the IFA-431 first power ramp was duplicated with IFA-432. Identical assemblies also reduce the uncertainties associated with assembly and rod power distributions. 
TABLE 2.1. Design Parameters and Instrumentation for IFA-431 and IFA-432 IFA-43) [Peak Power - $10 \mathrm{~kW} / \mathrm{ft}(328 \mathrm{~W} / \mathrm{cm})$ ]

\begin{tabular}{|c|c|c|c|c|c|c|c|c|c|c|c|c|}
\hline \multirow{2}{*}{$\begin{array}{l}\text { Rod } \\
\text { No. }\end{array}$} & \multirow{2}{*}{\multicolumn{2}{|c|}{$\begin{array}{c}\text { Diameter } \\
\text { Pellet }\end{array}$}} & \multirow{2}{*}{\multicolumn{2}{|c|}{$\begin{array}{c}\text { Cold } \\
\text { Diametral } \\
\text { Gap }(a)\end{array}$}} & \multicolumn{3}{|c|}{ Fuel } & & \multicolumn{4}{|c|}{ Instrumentation } \\
\hline & & & & & $\begin{array}{l}\text { Fill } \\
\text { Gas }\end{array}$ & $\begin{array}{c}\text { Density } \\
y T D\end{array}$ & $\begin{array}{c}\text { Fue } \\
\text { Type }\end{array}$ & $\begin{array}{r}\text { Burnup } \\
\text { MWd/MTM }\end{array}$ & Temper & ature & & $\begin{array}{l}\text { Cladding } \\
\text { Length }\end{array}$ \\
\hline 1 & 0.4205 & 10.681 & 0.009 & 0.229 & He & 95 & Stable & 4,000 & $T C^{(c)}$ & TC & $P_{T}$ (d) & $E S^{(e)}$ \\
\hline 2 & 0.4145 & 10.528 & 0.015 & 0.331 & He & 95 & Stable & 4,000 & TC & $T C$ & -- & ES \\
\hline 3 & 0.4275 & 10.858 & 0.002 & 0.051 & $\mathrm{He}$ & 95 & Stable & 4,000 & $T C$ & TC & -- & ES \\
\hline 4 & 0.4205 & 10.681 & 0.009 & 0.229 & Xe & 95 & Stable & 4,000 & TC & $T C$ & -- & ES \\
\hline 5 & 0.4205 & 10.681 & 0.009 & 0.229 & $\mathrm{He}$ & 92 & Stable & 4,000 & TC & TC & PT & ES \\
\hline 6 & 0.4205 & 10.681 & 0.009 & 0.229 & $\mathrm{He}$ & 92 & Unstable & 4,000 & TC & TC & PT & ES \\
\hline
\end{tabular}

IFA-432 [Peak Power - $15 \mathrm{~kW} / \mathrm{ft}(492 \mathrm{~W} / \mathrm{cm})]$

\begin{tabular}{|c|c|c|c|c|c|c|c|c|c|c|c|}
\hline & 0.4205 & 10.681 & 0.009 & 0.229 & $\mathrm{He}$ & 35 & Stable & 20,000 & $T C$ & $T C$ & PT \\
\hline & 0.4145 & 10.528 & 0.015 & 0.381 & $\mathrm{He}$ & 95 & Stable & $4,000^{(f)}$ & $u T^{(g)}$ & $T C$ & -- \\
\hline & 0.4265 & 10.833 & 0.003 & 0.076 & $\mathrm{He}$ & 95 & Stable & $4,000^{(f)}$ & TC & $\mathrm{TC}$ & -- \\
\hline & 0.4205 & 10.681 & 0.009 & 0.229 & $x e$ & 95 & Stable & $4,000^{(f)}$ & TC & TC & -- \\
\hline & 0.4205 & 10.681 & 0.009 & 0.229 & $\mathrm{He}$ & 92 & Stable & 20,000 & TC & $T C$ & PT \\
\hline & 0.4205 & 10.681 & 0.009 & 0.229 & $\mathrm{He}$ & 92 & Unstable & 20,000 & TC & $\mathrm{TC}$ & PT \\
\hline & 0.4145 & 10.528 & 0.015 & 0.381 & $\mathrm{He}$ & 95 & Stable & 16,000 & -- & -- & -- \\
\hline & 0.4205 & 10.631 & 0.009 & 0.229 & $\mathrm{He}$ & 95 & Stâble & 16,000 & -- & -- & -- \\
\hline & 0.4225 & 10.732 & 0.007 & 0.179 & $\mathrm{He}$ & 95 & Stable & 16,000 & -- & -- & -- \\
\hline
\end{tabular}

(a) Cladding for all rods has an OD of $0.5035 \mathrm{in.}(12.789 \mathrm{~mm})$ and an $1 D$ of $0.4295 \mathrm{in}$. (10.909 $\mathrm{mm})$. Diametral gao is cladding ID minus pellet diameter.

(b) With respect to in-reactor densification.

(c) $T C=$ Thermocouple

(d) $P T=$ Pressure Transducer

(e) $E S=$ Elongation Sensor

(f) Removable rods replaced by Rods 7,8 , and 9 .

(g) $U T=U 1$ trasonic Thermometer

The power profile in the Halden BWR (Figure 2.1) was also considered dur- . ing the design. The top of the rod was placed at the peak, which forced the bottom of the rod to operate at 70 to $80 \%$ of the peak rod power. To take advantage of the power distribution, thermocouples were placed in the top and bottom of each rod. Previously, no tests had been run in Halden with thermocouples penetrating both endcaps; however, Halden stafi were able to develop a workable design. Thermocouples in both ends allow modelers to check the ability of various codes to extrapolate over a short power range within the same rod. If a code cannot perform these calculations adequataly, calculations of the temperature distribution over an $\sim 4 \mathrm{~m}$ fuel length are also suspect. 


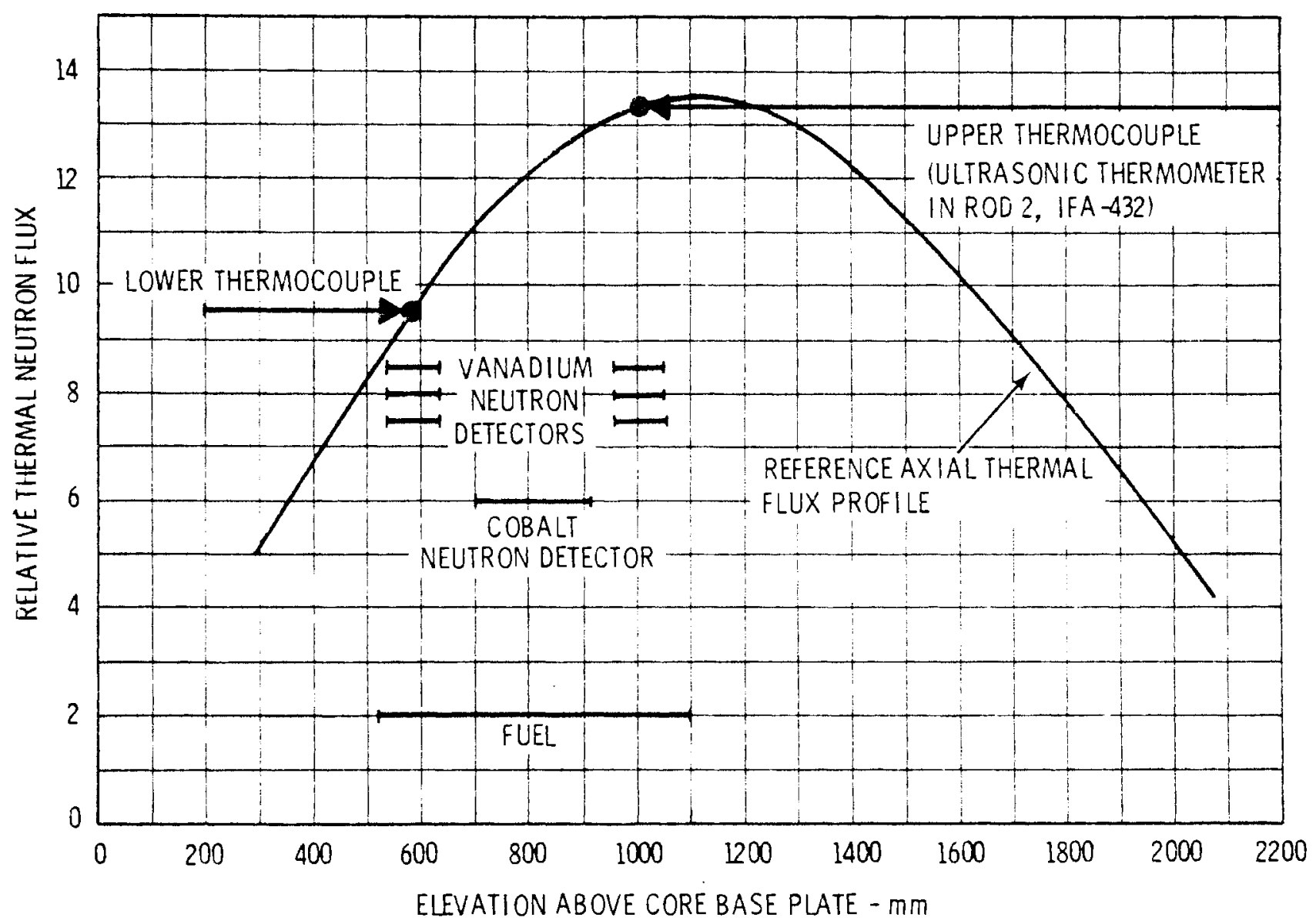

FIGURE 2.1. Arrangement of Temperature Sensors, Neutron Detectors, and Fuel Relative to Reference Axial Thermal Flux Profile, IFA-431 and 432

Using two thermocouples per rod allows modelers to interpolate between $\sim 7 \mathrm{~kW} / \mathrm{ft}$ $(230 \mathrm{~W} / \mathrm{cm})$ and $15 \mathrm{~kW} / \mathrm{ft}(492 \mathrm{~W} / \mathrm{cm})$ with tie points at $210 \mathrm{~kW} / \mathrm{ft}(328 \mathrm{~W} / \mathrm{cm})$ and $212 \mathrm{~kW} / \mathrm{ft}(394 \mathrm{~W} / \mathrm{cm})$. Single thermocouples would have provided data at only $10 \mathrm{~kW} / \mathrm{ft}(328 \mathrm{~W} / \mathrm{cm})$ and $15 \mathrm{~kW} / \mathrm{ft}(492 \mathrm{~W} / \mathrm{cm})$. Data is obtained, as a result of reactor cycling, over the entire range of 0 to $15 \mathrm{~kW} / \mathrm{ft}(492 \mathrm{~W} / \mathrm{cm})$. This should span most of the anticipated normal operating powers in commercial plànts.

Reference points with commercial plants and other fuel research programs were also developed. Selection of the BWR-6 fuel geometry, procurement of commercial quality tubing, and selection of assembly powers connects the data to commercial plants. Some of the cladding procured for this program was shipped to EG\&G (INEL) for use in their Halden tests. Both programs also used the 
same starting powder for fuel manufacture. The length of the rods in both IFA431 and 432 were designed to be compatible with the Power Burst Facility (PBF) test trains; we11-characterized irradiated fuel is needed for a number of tests in the PBF program. Some of the fuel structures were similar to those investigated in the EEI/EPRI UO 2 fuel densification study, ( $(i)$ to provide a reference point to a much larger structural characterization program.

The correct assessment of rod powers and the distribution of power within the rods is of utmost importance to assure the best possible thermal data. To attain this, seven neutron sensors were placed in each assembly (Figure 2.2); one in the center of the assembly, three at the top plane of thermocouples, and three at the bottom plane of thermocouples. An extensive calibration of the Vanadium sensors is conducted during the initial startup of any assembly. In addition, Rod 3 (0.051 and $0.076 \mathrm{~mm}$ gap for IFA-431 and 432, respectively) was included as an internal standard. The small gap was closed at power; thus, the temperature gradient across the gap was minimized. Since the coolant temperature and fuel centerline temperatures were known, an independent check of rod power at both the top and bottom planes in the assembly was obtained. Rod powers and fuel temperatures in both assemblies were compared to assure consistent data. Each rod had a cladding elongation sensor; Rods 1, 5, and 6 had fission gas pressure transducers.

Design assembly burnups for IFA-431 were set at 4000 MWd/MTM to ensure complete densification, minimize fuel volume changes induced by fission product swelling, and to retain the option of PIE confirmation of gap closure data obtained from low burnup rods. The second assembly, IFA-432, is a sibling to IFA-431. Three major differences exist in the design parameters; the design power is $15 \mathrm{~kW} / \mathrm{ft}(492 \mathrm{~W} / \mathrm{cm})$, the goal burnup is 20,000 MWd/MTM, and the assembly contains an option to replace three rods at $4000 \mathrm{MWd} / \mathrm{MTM}$, allowing cross correlation with three identical rods in IFA-431.

Table 2.2 illustrates the amount of cross correlation that is possible. In addition to the rod-to-rod comparisons, top-to-bottom comparisons can be made in each rod, and separate effects as a function of burnup and power can also be evaluated. 


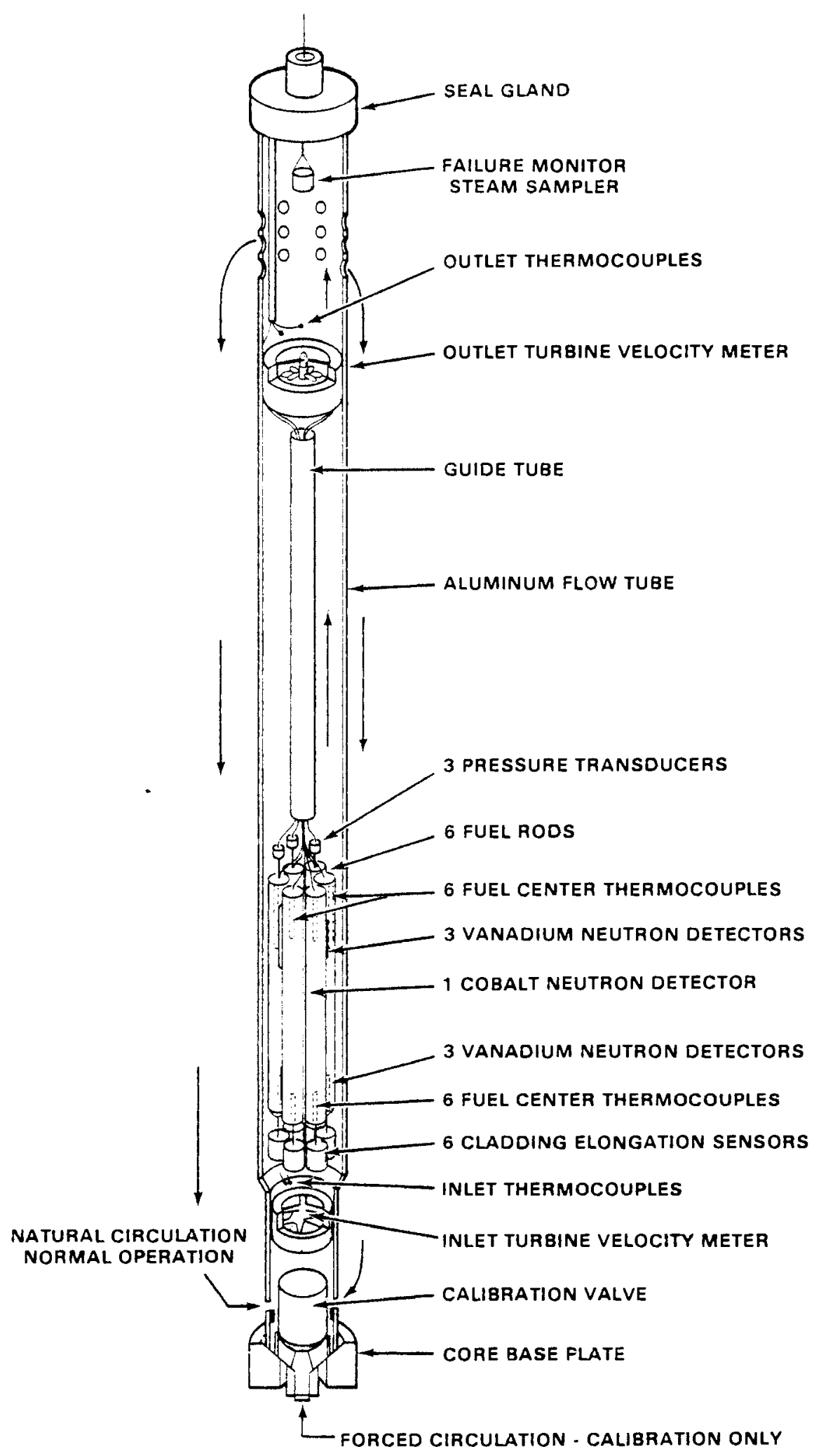

FIGURE 2.2. Schematic of Instrumented Fuel Assembly IFA-432 
TABLE 2.2. Cross-Correlation Matrix

\begin{tabular}{|c|c|c|c|c|c|c|c|c|c|}
\hline $\begin{array}{c}\text { Rod } \\
\text { Number } \\
\end{array}$ & $\begin{array}{r}\text { Gap } \\
\text { Size } \\
\end{array}$ & $\begin{array}{c}\text { Fuel } \\
\text { Relocation } \\
\end{array}$ & $\begin{array}{c}\text { Fuel } \\
\text { Eccentricity }\end{array}$ & $\begin{array}{c}\text { Fue } 1 \\
\text { Stability }\end{array}$ & $\begin{array}{c}\text { Gas } \\
\text { Composition }\end{array}$ & $\begin{array}{l}\text { Fuel } \\
\text { Density }\end{array}$ & $\begin{array}{l}\text { Rod } \\
\text { Powers } \\
\end{array}$ & $\begin{array}{c}\text { Rod } \\
\text { Pressures } \\
\end{array}$ & $\begin{array}{c}\text { Dynamic } \\
\text { Temperature } \\
\end{array}$ \\
\hline $1(9-\mathrm{He}-95-5)^{(a)}$ & $x$ & $x$ & & & $x$ & $x$ & & $x$ & $x$ \\
\hline $2(15-\mathrm{He}-95-\mathrm{S})$ & $x$ & $x$ & & & & & & & $x$ \\
\hline $3(2-\mathrm{He}-95-\mathrm{S})$ & $x$ & $x$ & & & & & $x$ & & $x$ \\
\hline $4(9-x e-95-5)$ & & $x$ & $x$ & & $x$ & & & & $x$ \\
\hline $5(9-\mathrm{He}-92-\mathrm{S})$ & & $x$ & & $x$ & & $x$ & & $x$ & $x$ \\
\hline $6(9-\mathrm{He}-92-\mathrm{J})$ & & $x$ & & $x$ & & & & $x$ & $x$ \\
\hline
\end{tabular}

(a) (9-He-95-5) - the first character identifies the rod, the second the nominal diametral gap in mils, the third the fill gas, the fourth the fuel density, and the fifth stable (S) or unstable (U) fuel.

\subsection{TEST FACILITY}

The Halden Boiling Water Reactor (HBWR) uses natural circulation of heavy water for cooling. Reactor operating data are shown in Table 2.3.

\section{TABLE 2.3. Operating Data for the HBWR}

Power Level

Reactor Pressure

Heavy Water Saturation Temp.

Plenum Inlet Temp.

Thermal Flux

Fast Flux (>1 MeV)

Average Fuel Power Density
$12 \mathrm{MW}$

500 psi (34 atm)

$464^{\circ} \mathrm{F}\left(240^{\circ} \mathrm{C}\right)$

$459^{\circ} \mathrm{F}\left(237^{\circ} \mathrm{C}\right)$

$23 \times 10^{13} \mathrm{n} / \mathrm{cm}^{2}-\mathrm{sec}$

$\sim 5 \times 10^{11} \mathrm{n} / \mathrm{cm}^{2}-\mathrm{sec}$

$14.8 \mathrm{~W} / \mathrm{g}$

A schematic of the plan view of the HBWR core loading in November 1975 is shown in Figure 2.3. The locations of IFA-43I and IFA-432 are also indicated. Both half-length assemblies were charged into the bottom half of the core (Figure 2.1).

\subsection{FUEL ROD THERMAL DESIGN PARAMETERS}

\subsubsection{Contemporary Rod (Rod 1)}

It was important to obtain as many reference points as possible to cross correlate the data and provide avenues to extrapolate these test results to U.S. commercial nuclear fuel. Accordingly, the primary reference selected con- 


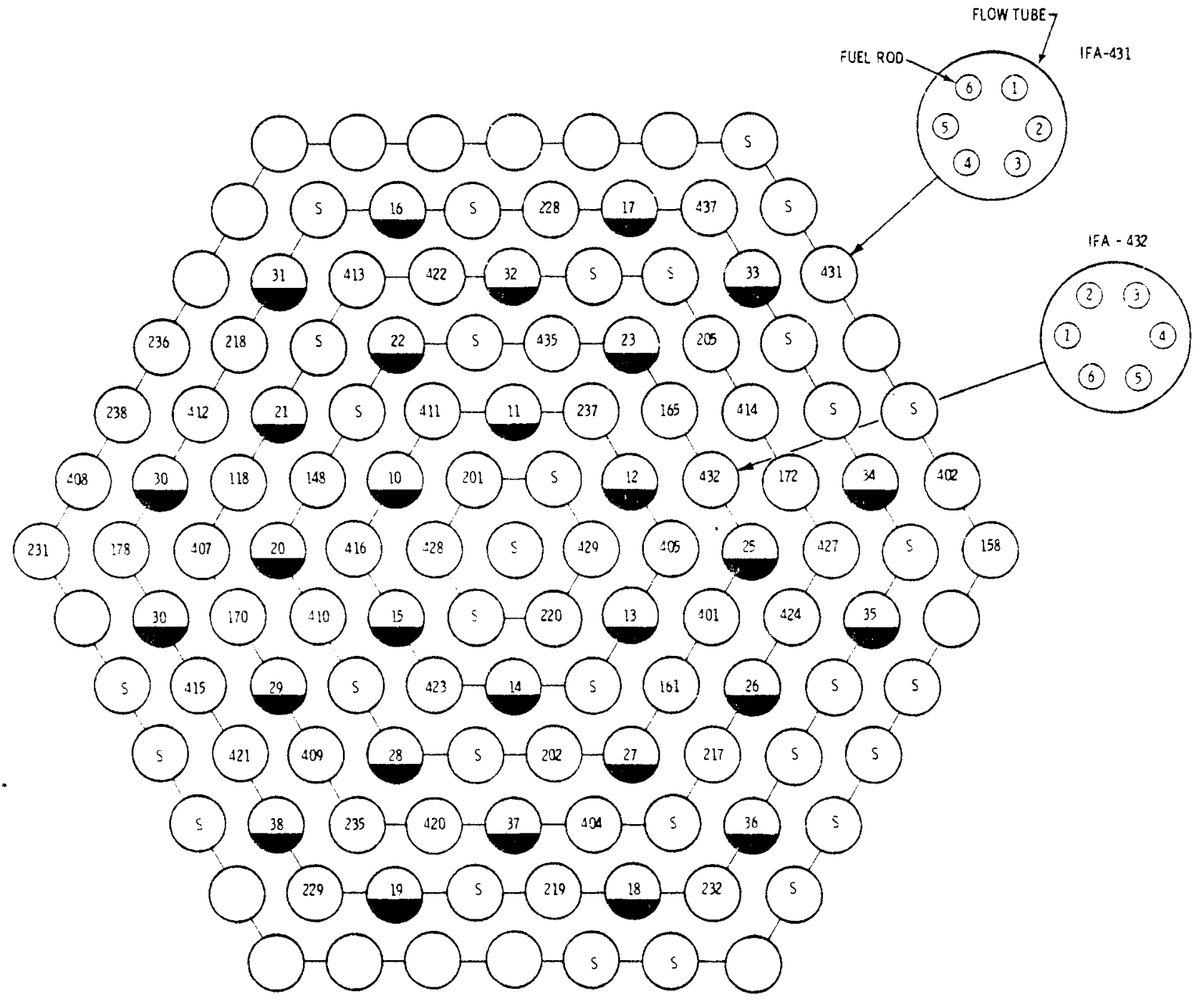

FIGURE 2.3. IFA-431 and IFA-432 Arrangements in the Flow Channel 
sists of a BWR-6 geometry, a 95\% TD peliet, a 0.009 in. (0.229 mm) diametral gap, and helium fill gas. In addition to minimizing the difficulty of extrapolating the data from the Halden tests to commercial plants, the larger BWR pellets tend to reduce perturbations induced by thermocouples. Cladding procurement problems were also minimized since atypical cladding dimensions are difficult to obtain from commercial sources. The 95\% TD fuel density was selected to assure a densification-resistant fuel, provide a density typical of 1974 vintage fuel designs, and provide a reference point to previous thermal conductivity measurements on unirradiated fuel. Rod 1 in each assembly fulfills the above objectives set for the experiment reference.

\subsubsection{Simulated Instantaneous Densification (Rod 2)}

The major impact of fuel densification (2) was the assumption that the fuel underwent isotropic shrinkage directly proportional to the difference between the fabricated density and the terminal density. For example, a $93.5 \%$ TD BWR fuel pellet $(0.488-i n$. [12.395 mm] diameter) would shrink 0.00488 in. (0.124 mm) if the NRC criteria of a $95.5 \%$ TD terminal density were applied. Most BWR fuel rods of the above pellet size would contain an initial diametral gap of 0.011 to $0.012 \mathrm{in.}(0.279-0.305 \mathrm{~mm})$. Consequently, the shrinkage induced by densification would create a diametral gap of about 0.015 to $0.017 \mathrm{in}$. (0.381-0.432 mm). Consideration of tolerances (i.e., $\pm 0.0015 \mathrm{in.}$ ) on the initial gap resulted in even larger gaps. Until data became available from the EEI/EPRI UO 2 densification program, $(1,3)$ the Halden program, (4) and individual fuel vendor programs, instantaneous densification was assumed. (2) That is, a gap of $0.016 \mathrm{in}$. (0.406 mm), for the example above, was assumed to exist as soon as the plant reached full power.

Accordingly, Rod 2 was designed with a $0.015-i n .(0.381 \mathrm{~mm})$ diametral gap to simulate instantaneous densification. The $0.015-i n$. gap in the BWR-6 geometry (0.4205-in. [10.681 mm] reference pellet diameter) yields a gap/diameter $(G / D)$ ratio of 0.036 which approximates the $G / D$ ratios typically postulated for densified fuel in either the older BWR or PWR fuel designs.

\subsubsection{Internal Reference (Rod 3)}

Rod 3 was designed primarily for an independent check of rod powers. This rod contained 95\% TD fuel with an initial diametral gap of $0.002 \mathrm{in} .(0.051 \mathrm{~mm})$ $(0.003$ in. in IFA-432). These gaps were chosen to provide good contact at power 
and yet minimize the potential for cladding failure induced by mechanical interaction. The small gap was closed at power, which produced very high gap conductances and thus minimized the temperature gradient across the fuel-cladding interface. Minimizing the temperature gradients across the gap is a good check of the effective thermal conductivity of the fuel in-reactor. Since cladding temperatures are calculable, and thermocouples indicated the fuel centerline temperatures, rod powers in the assembly could be checked. The technique that was used was based on the conductivity integral. (5)

A series of controlled power drops were requested for each assembly. Three cycles in each series consisted of a 20\% linear drop in power over a 60-sec time interval. These tests yielded transient gap conductance data for comparison with the steady-state measurements. (6) Steady-state values were availabie for powers at the beginning and end of a ramp for boundary conditions. Data from the transient provided a cross-check on the steady-state data, as well as a data base for developing or verifying portions of the transient gap conductance models needed to satisfy the final acceptance criteria for emergency core cooling system.

Rod 3 also provided an upper bound for gap conductance.

\subsubsection{Effects of Xenon and Eccentricity (Rod 4)}

Previous experiments have indicated that gap conductances are not as sensitive to different fill gas compositions as some models would predict. In particular, Cohen et al., (7) Horn ${ }^{(8)}$ and Lawrence et al., (9) have reported that large portions of Xenon must be mixed with helium before significant changes in gap conductance can be detected. In addition, analytical work had indicated that asymmetric pellet cladding gap geometries could have a significant effect on fuel temperatures and gap conductances. (10) The experimental resolution of the above was considered in the design of the NRC-RSR/PNL Halden assemblies IFA-431 and IFA-432.

To understand the anomalous thermal behavior of Xenon-filled rods, we designed a rod (Number 4) that contained $95 \%$ TD stable fuel, a 0.009-in. $(0.229 \mathrm{~mm})$ diametral gap, and Xenon fill gas. Instrumented portions of the fuel column were mechanically constrained to be held concentric (near the top) 
and eccentric (near the bottom) of the rod (Figure 2.4). This was accomplished using the fuel centerline thermocouples, molybdenum rods, and oversized pellets. These oversized pellets were of the same desian as Rod 3 of each respective assembly. They had flats ground on the periphery to allow axial fill gas communication.

Results of the analysis of the data from this rod may be seen in Reference 11 .

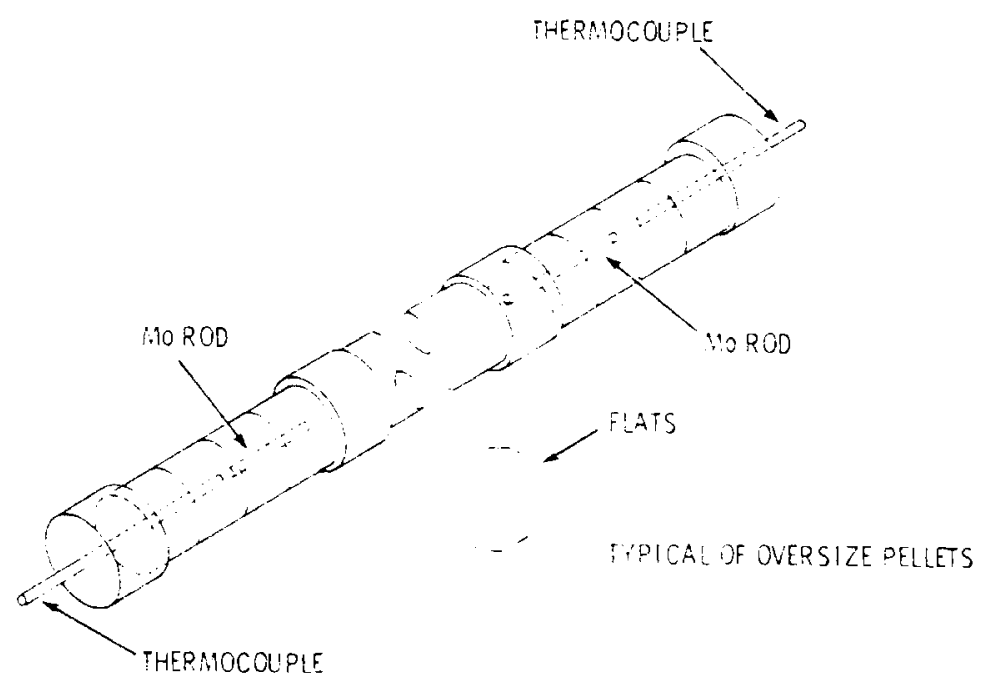

FIGURE 2.4. Schematic of Rod 4 Fuel Column

\subsubsection{Verification of Fuel Stability (Rod 5)}

Results of recent experimental work $(1,12)$ provide the technology required to fabricate low density (e.g., 92\% TD) fuel with various degrees of stability or resistance to irradiation-induced densification. New commercial designs under consideration will use low density, stable,fuel. Rod 5 contained 92\% TD stable fuel and provided one means of verifying recent fuel fabrication technoiogy with regard to the effects of fuel structures on irradiation temperatures. This rod will also be a cross-correlation with Rod 6 .

\subsubsection{Densification Kinetics (Rod 6)}

Rod 6 contains $92 \%$ TD unstable fuel and a 0.009 in. $(0.229 \mathrm{~mm})$ gap. Unstable fuel with similar structure ${ }^{(1,12)}$ underwent significant densification. 
Densification models utilizing kinetic information obtained from $\mathrm{Halden}^{(4)}$, terminal densities derived from the EEI/EPRI studies, (1) and resintering tests were developed to simulate time-dependent densification. Data from Rod 6 will be used to verify NRC densification models $(13,14)$ and provide a check of applicant-supplied models.

\subsection{FUEL AND CLADDING PRECHARACTERIZATION}

Extensive precharacterization of the fuel and cladding was essential to assure quality data and to reduce calculational uncertainties. Since this is presented elsewhere, (15) only the main objectives will be discussed here.

The previous discussion describes the importance of knowing the correct power distribution. Thermal diffusivity measurements were made on each fuel type up to $1600^{\circ} \mathrm{C}$. This spans most of the fuel volume in either test. From previous experimental work, the heat capacity and density were obtained to calculate the thermal conductivity of the fuel as a function of temperature. Substitution of these data for the Lyons $(16)$ thermal conductance equation used in the GAPCON-THERMAL-2 (17) pretest predictions improved the power calibration calculation using Rod 3. In addition, the thermal diffusivity data reduced the uncertainty associated with calculating gap conductances from the experimental data. Figure 2.5 shows the differences between the Lyons and IFA-432 precharacterization fuel thermal conductivities.

Establishing the initial dimensions and void volumes within the pins was also an essential part of assessing all thermal calculations. Consequently, the lengths and diameters of each pellet and the cladding for each rod were measured. Each pellet was identified with a unique number to trace pellet types and position within the rod (see Appendix A). With this information the axial distribution of gap volume and the plenum volume were obtained with considerable accuracy. Pellet and cladding roundness profiles were also obtained to illustrate the departure from ideal coaxial cylinders used in most computer code models.

Geometric densities were determined for all pellets and immersion densities for a significant fraction of the pellets. A correlation was developed 


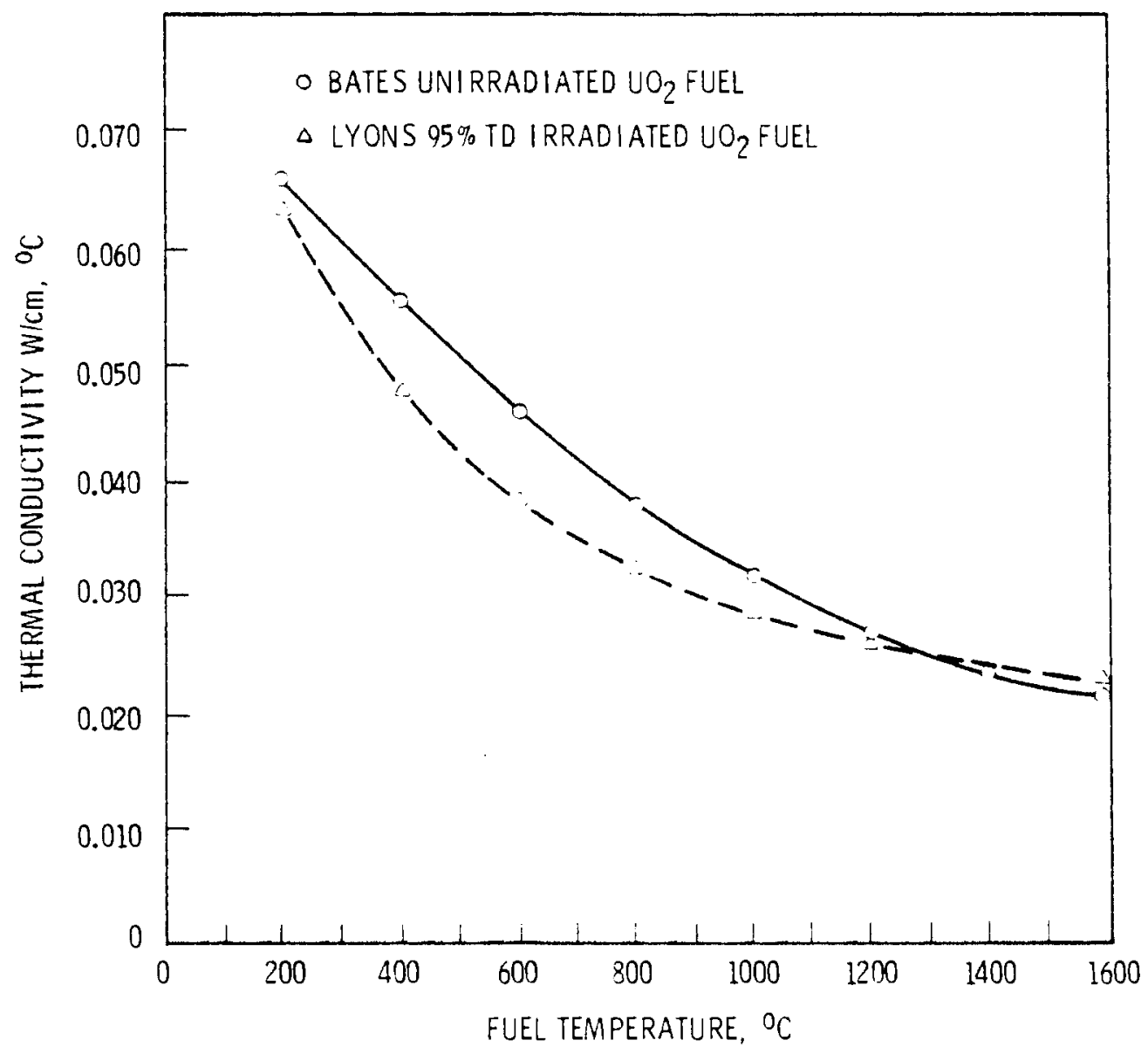

FIGURE 2.5. IFA-432 Fuel Therma conductivity, 95\% TD Stable, Rods 1, 2, 3, 4

relating immersion density to geometric densities. These data were used in two ways, 1) in a correction to rod powers caused by differences in mass distribution and 2) in the verification of NRC resintering models used to characterize fuel's propensity to densify. Resintering tests conducted on each fuel type are discussed in Reference 15.

The EEI/EPRI $\mathrm{UO}_{2}$ densification program ${ }^{(1)}$ demonstrated the importance of pore-size distribution measurements in characterizing the stability of various fuel types. We wanted to assure that the 95\% TD stable fuel types were indeed stable. On the other hand, we wanted the $92 \%$ TD unstable fuel to undergo large 
amounts of densification in-reactor to determine the effects on fuel temperatures. Both fuel densities and pora-size distribution will be measured in a post-irradiation examination (PIE) for Rods 1, 5 and 6 at Harwell, UK. Archive pellets from each fuel type were retained to provide a means of reducing variances associated with potential differences in examination techniques used in the pre-and post-test measurements. 


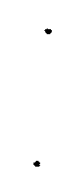




\subsection{DATA PRESENTATION}

Figures 3.1 through 3.226 show the in-reactor data collected from IFA-432 during its irradiation period from December 1975 to January 1978 plotted versus time by months. A11 data except the rod internal pressures were obtained by the Halden IBM/1800 on-line computer data acquisition system. Data densities (versus time) vary from 15 minutes to 12 hours, depending on whether the reactor was experiencing transient (startup, power cycling) or steady state conditions.

In each figure, the rod number for each curve appears in the upper left hand corner. The relative position of the rod number corresponds to the relative position of the curve in each figure.

\subsection{POWER HISTORIES}

Figures 3.1 through 3.120 show the power histories of the upper and lower thermocouple locations for all six rods. These values were deduced from the vanadium self-powered neutron detector readings after applying correction factors to account for local mass distribution, radial flux tilt, and axial flux shape. This is described in Appendix D.

Corrections were also made for the burnup dependent depletion of ${ }^{235} \mathrm{U}$, but no plutonium buildup was considered. Halden recommended the depletion correction that was used $(-.66 \%$ per 1000 MWd/MTM).

The neutron detector readings during transient periods have not been corrected for the response lag of the detector caused by incomplete saturation of the vanadium emitter. This lag amounts to about 5 minutes during a power ramp, or one-third of the normal data collection frequency.

\subsection{FUEL TEMPERATURE HISTORIES}

Figures 3.121 through 3.186 show the fuel centerline temperature histories for the upper and lower thermocouple locations for all six rods. These data were collected from the W $5 \%$ Re/W 26\% Re sheathed, grounded thermocouples inserted in each end of each rod (see Appendix B). The ultrasonic thermometer at the top of Rod 2 failed, and there is no data for this position. 
In using these data, the reader should be aware that some anomolies have been seen between upper and lower thermocouples in the same rod, suggesting that the fuel at one end of the rod may be relatively decoupled from that at the other end. Future PNL reports will discuss these data.

\subsection{CLADDING ELONGATION HISTCRIES}

Figures 3.187 through 3.209 show the cladding elongation histories for a 11 six rods. Each rod was equipped with an LVDT-type elongation sensor of Halden design to monitor length changes throughout life. The results of the analysis of these data are discussed in Reference 18.

\subsection{ROD INTERNAL PRESSURE HISTORIES}

Figures 3.210 through 3.226 show the internai gas pressure histories for Rods 1,5 and 6 throughout their irradiation history. These measurements were taken manually at various assembly powers using a null balance technique to complete an electrical connection in the diaphragm-type pressure transducers. The pressure readings were taken from the gas feed line that supplied the pressure to balance the transducer, and indicate gauge pressure. The data points connected by straight lines in Figures 3.210 through 3.226 indicate the number of times that the pressure data was manually collected throughout the life of the assembiy. Results of the analysis of these data will be discussed in future PNL reports.

\subsection{BURNUP}

Table 3.1 shows the calculated burnups at each thermocouple location for each rod. These are the local burnups at the end of each month of operation. They were calculated by numerically integrating the power history over time and then correcting for the depletion of ${ }^{235} \mathrm{U}$. Comparisons of this method and PIE data for Rod 6 of IFA-431 showed good agreement. 


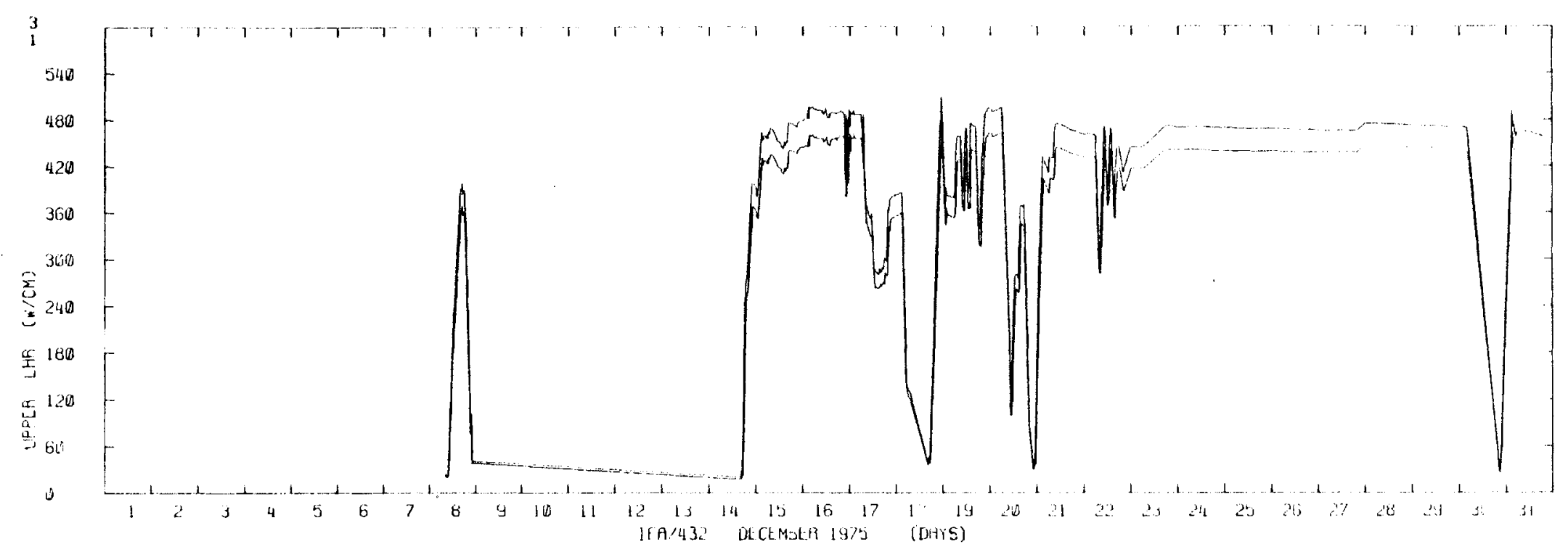

FIGURE 3.1. Local Linear Heat Ratings at Upper Thermocouple Locations for $\omega$
$\omega$
$\omega$ Rods 1,3 of IFA 432 - December 1975

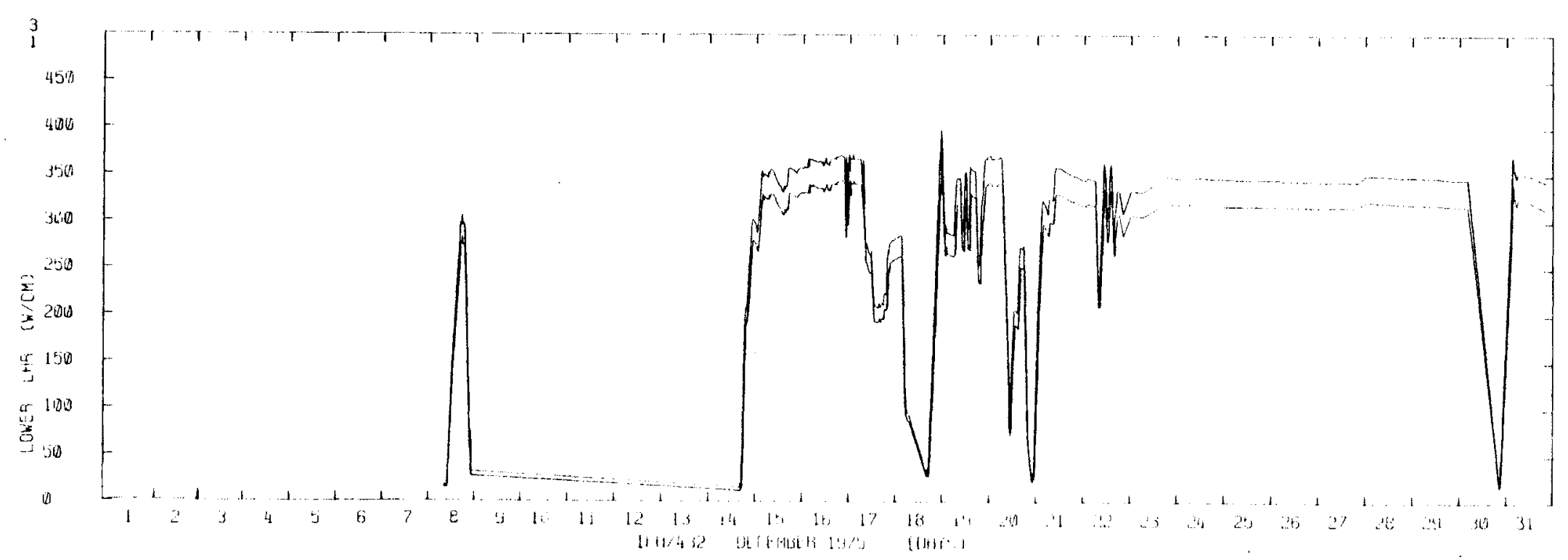

FIGURE 3.2. Local Linear Heat Ratings at Lower Thermocouple Locations for Rods 1,3 of IFA 432 - December 1975 


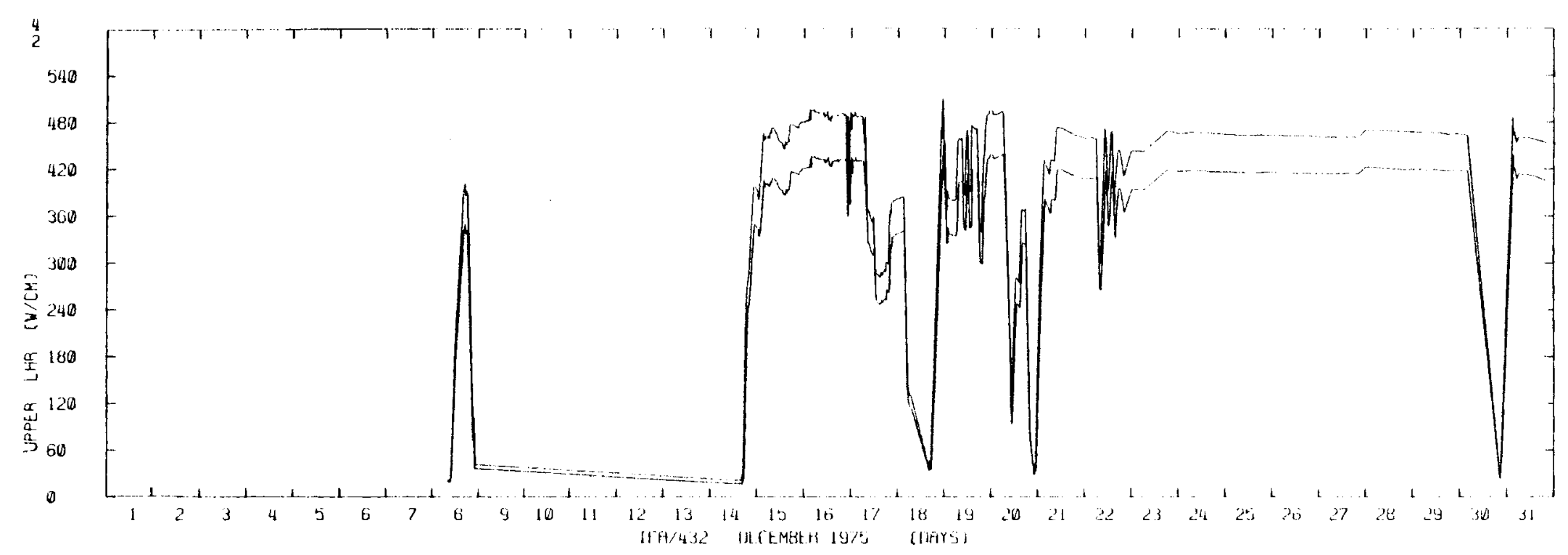

$\stackrel{\omega}{\vdots}$

FIGURE 3.3. Local Linear Heat Ratings at Upper Thermocouple Locations for Rods 2,4 of IFA 432 - Decenber 1975

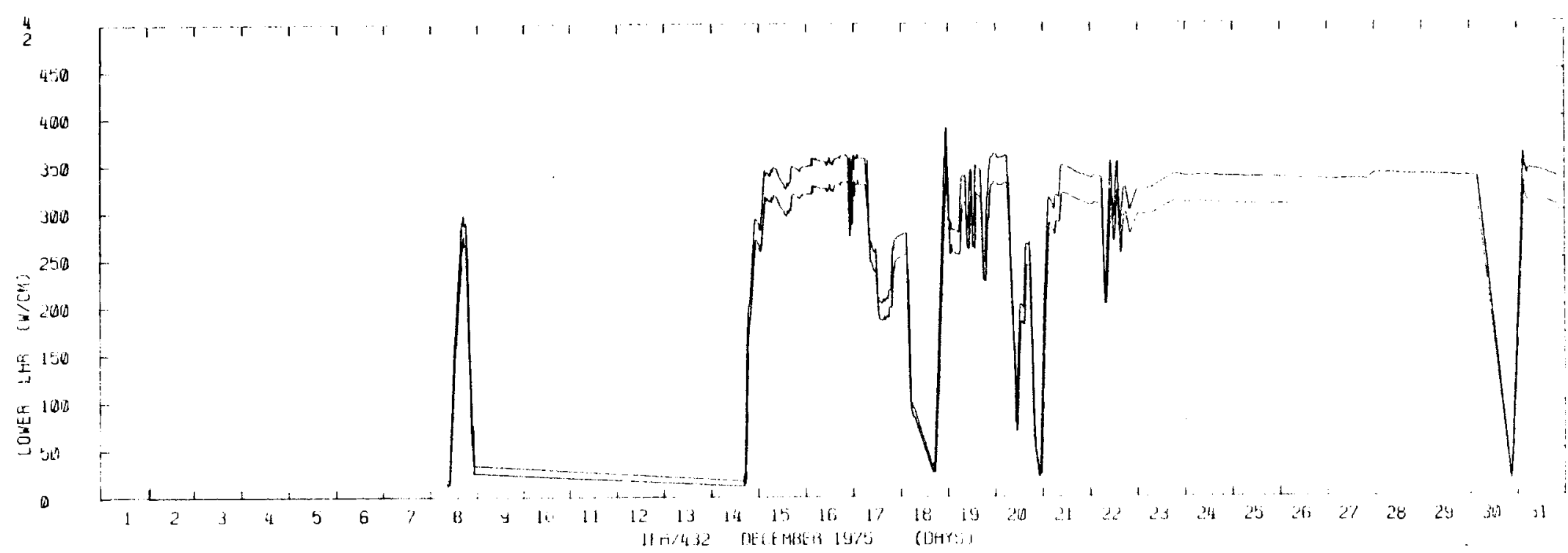

FIGURE 3.4. Local Linear Heat Ratings at Lower Thermocouple Locations for Rods 2,4 of IFA 432 - December 1975 


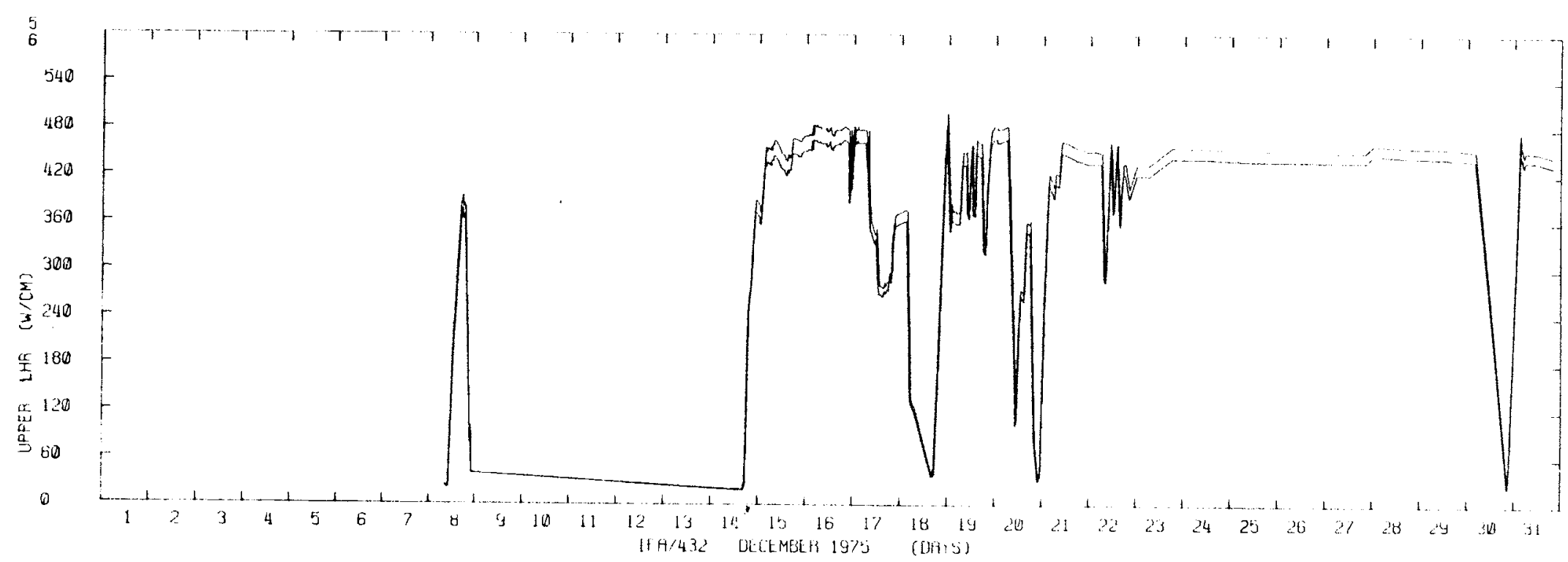

$\stackrel{\omega}{\dot{w}}$

FIGURE 3.5. Local Linear Heat Ratings at Upper Thermocouple Locations for Rods 5,6 of IFA 432 - December 1975

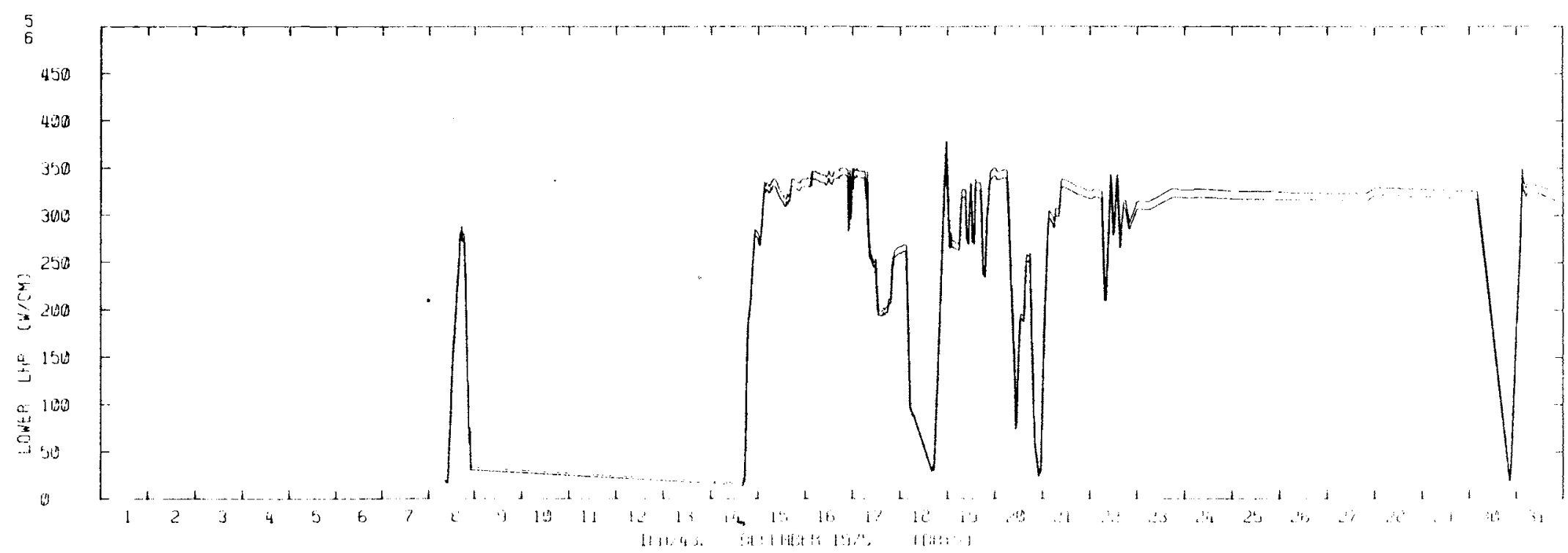

FIGURE 3.6. Local Linear Heat Ratings at Lower Thermocouple Locations for Rods 5,6 of IFA 432 - December 1975 


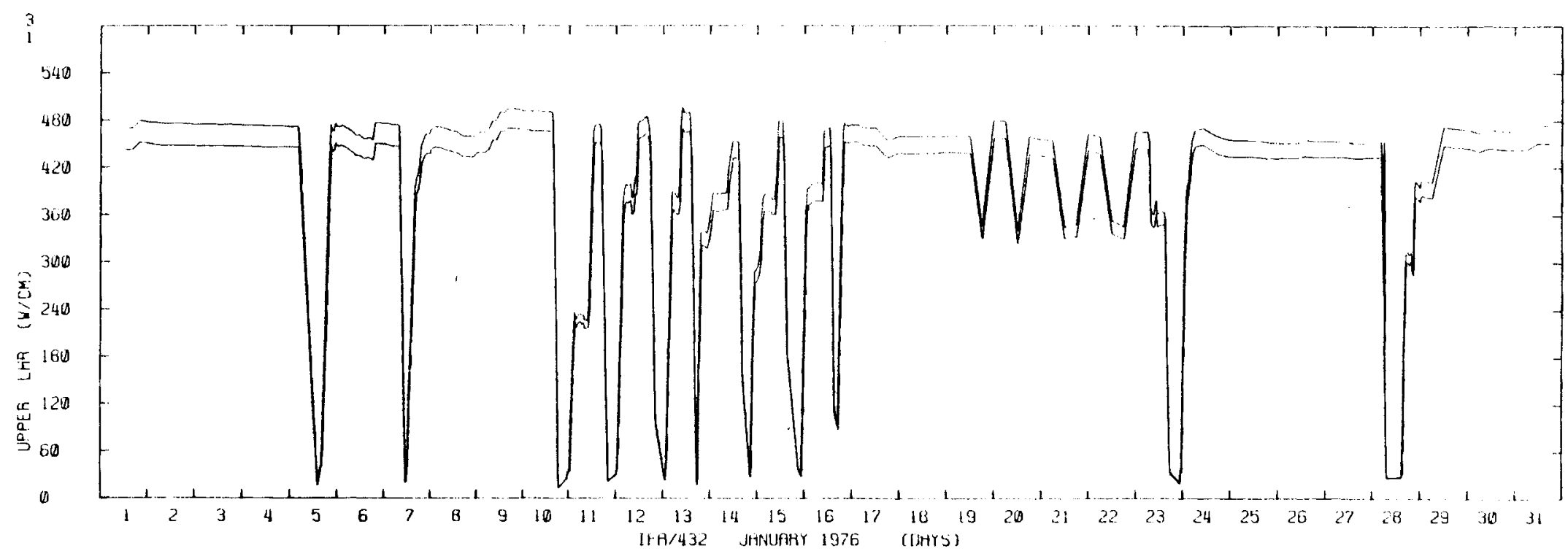

ó

FIGURE 3.7. Local Linear Heat Ratings at Upper Thermocouple Locations for Rods 1,3 of IFA 432 - January 1976

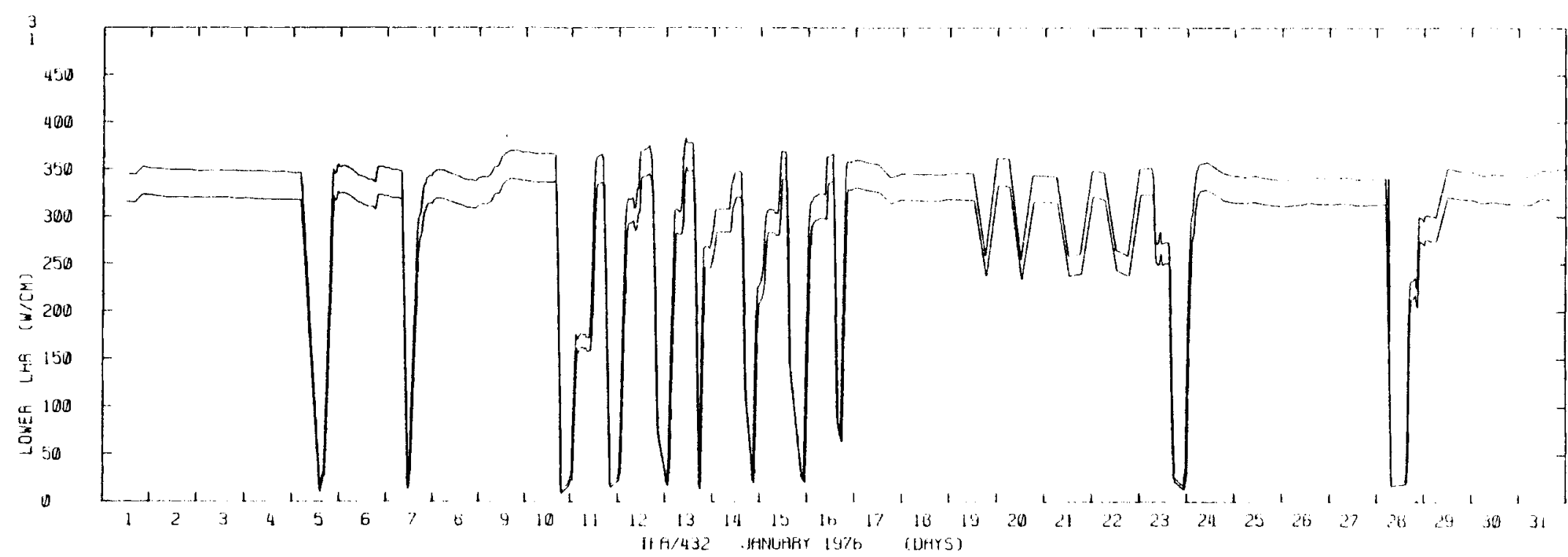

FIGURE 3.8. Local Linear Heat Ratings at Lower Thermocouple Locations for Rods 1,3 of IFA 432 - January 1976 


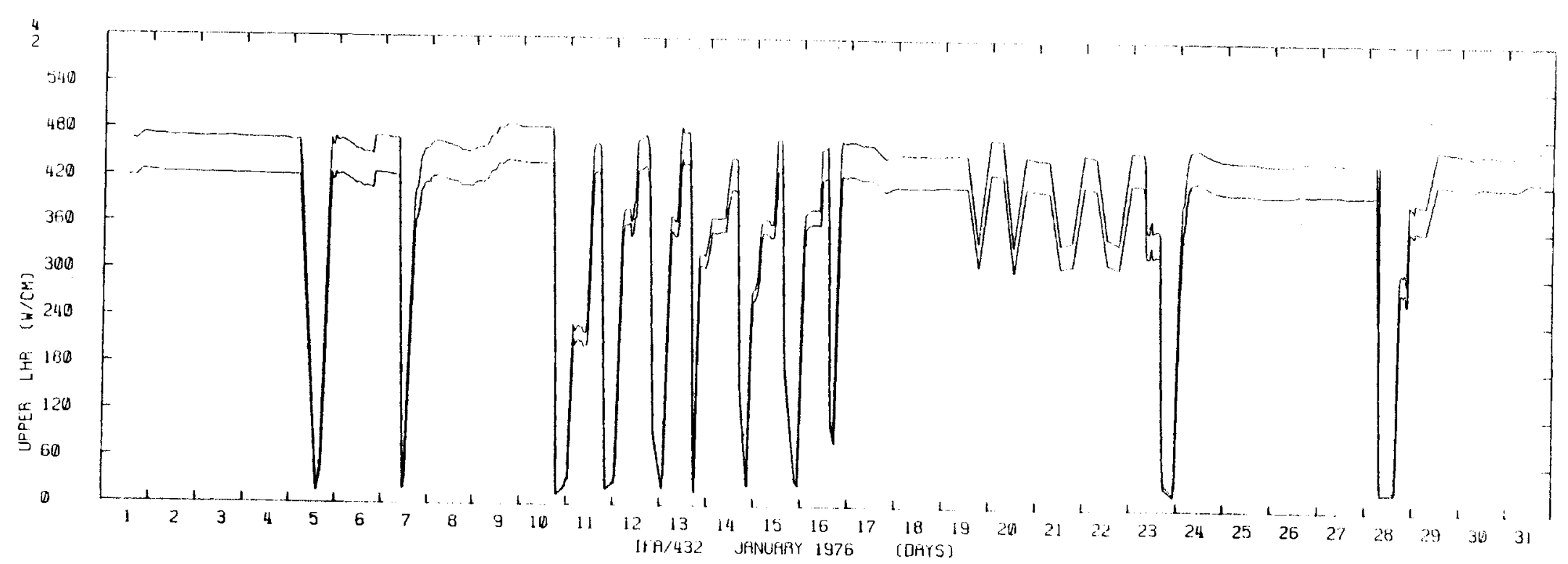

$\stackrel{\omega}{\longleftarrow}$

FIGURE 3.9. Local Linear Heat Ratings at Upper Thermocouple Locations for Rods 2,4 of IFA 432 - January 1976

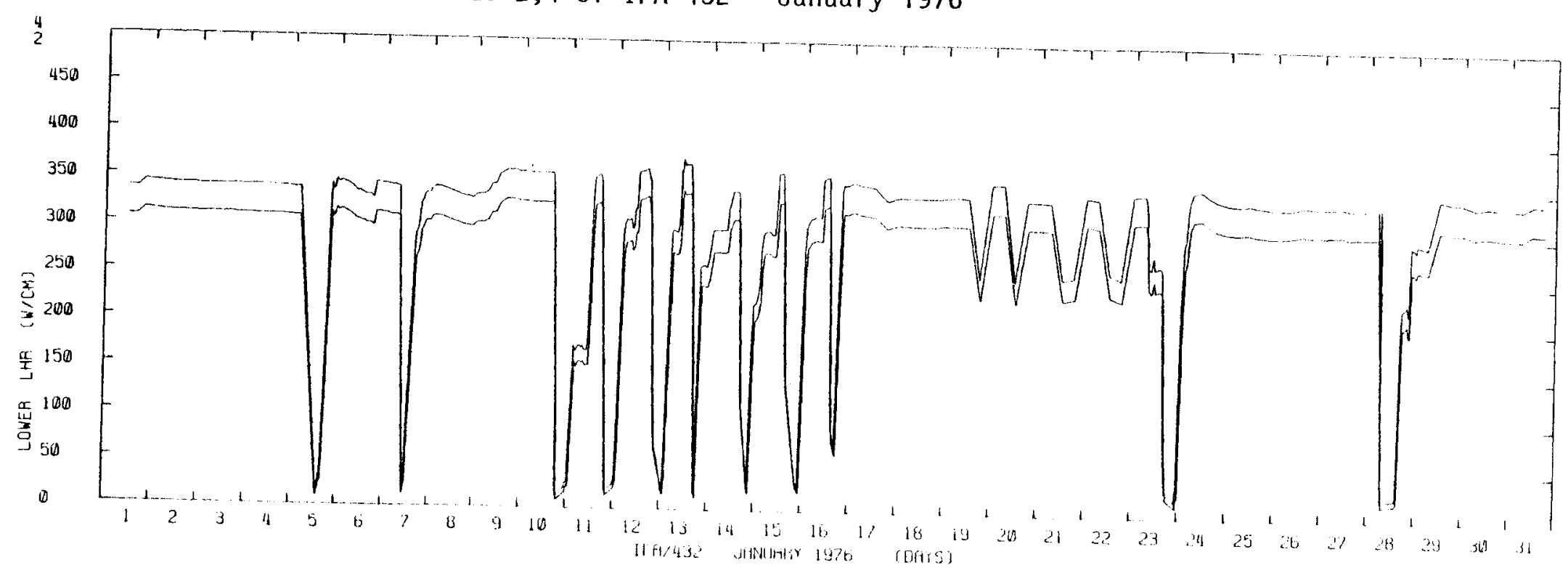

FIGURE 3.10. Local Linear Heat Ratings at Lower Thermocouple Locations for Rods 2,4 of IFA 432 - January 1976 


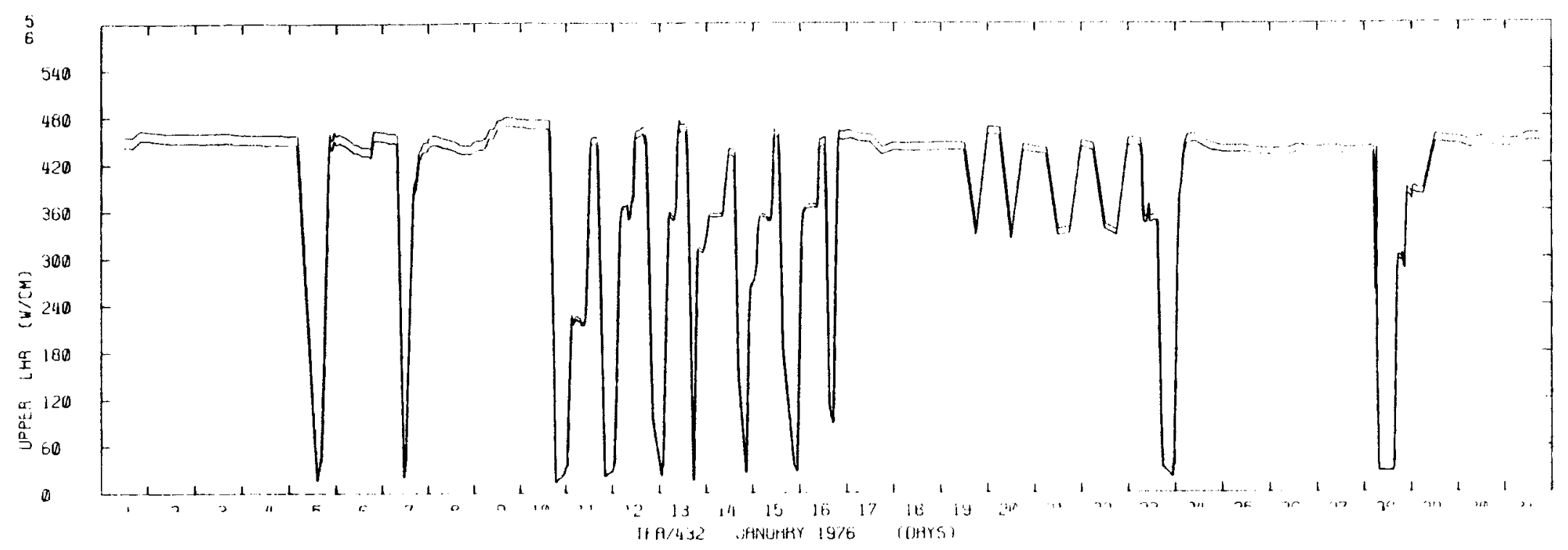

$\stackrel{\omega}{\infty}$

FIGURE 3.11. Local Linear Heat Ratings at Upper Thermocouple Locations for Rods 5,6 of IFA 432 - January 1976

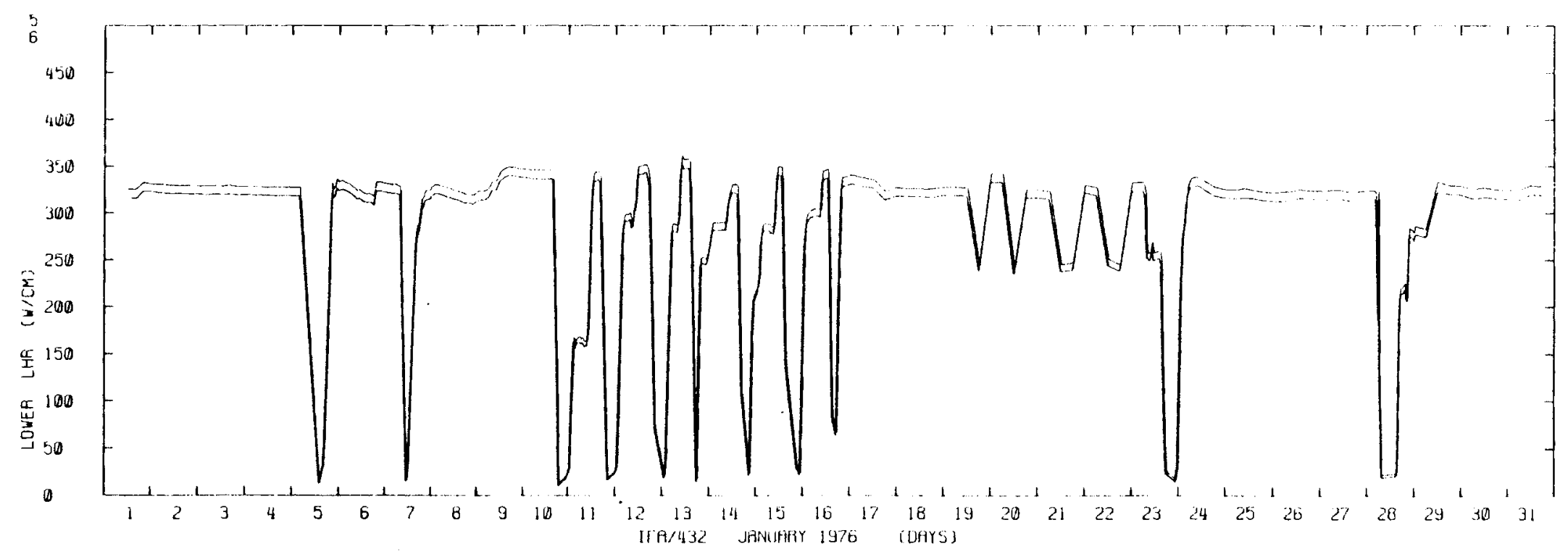

FIGURE 3.12. Local Linear Heat Ratings at Lower Thermocouple Locations for Rods 5,6 of IFA 432 - January 1976 


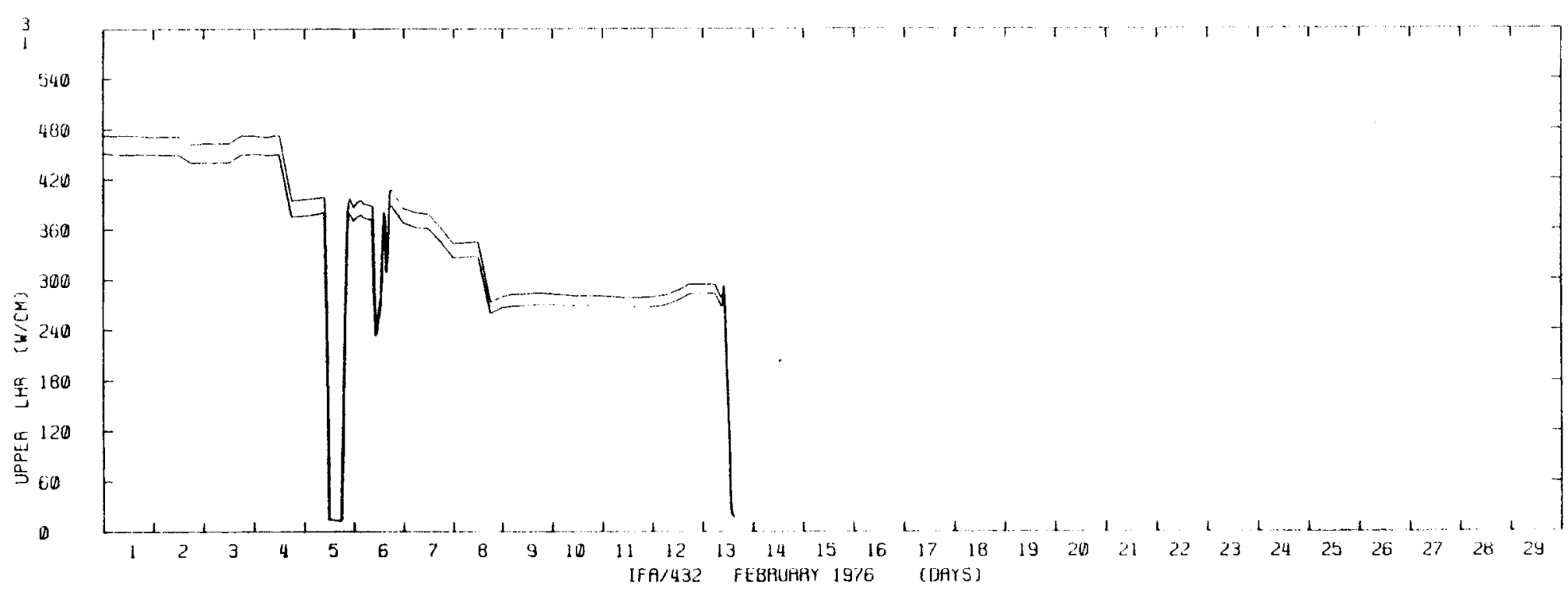

FIGURE 3.13. Local Linear Heat Ratings at Upper Thermocouple Locations for Rods 1,3 of IFA 432 - February 1976

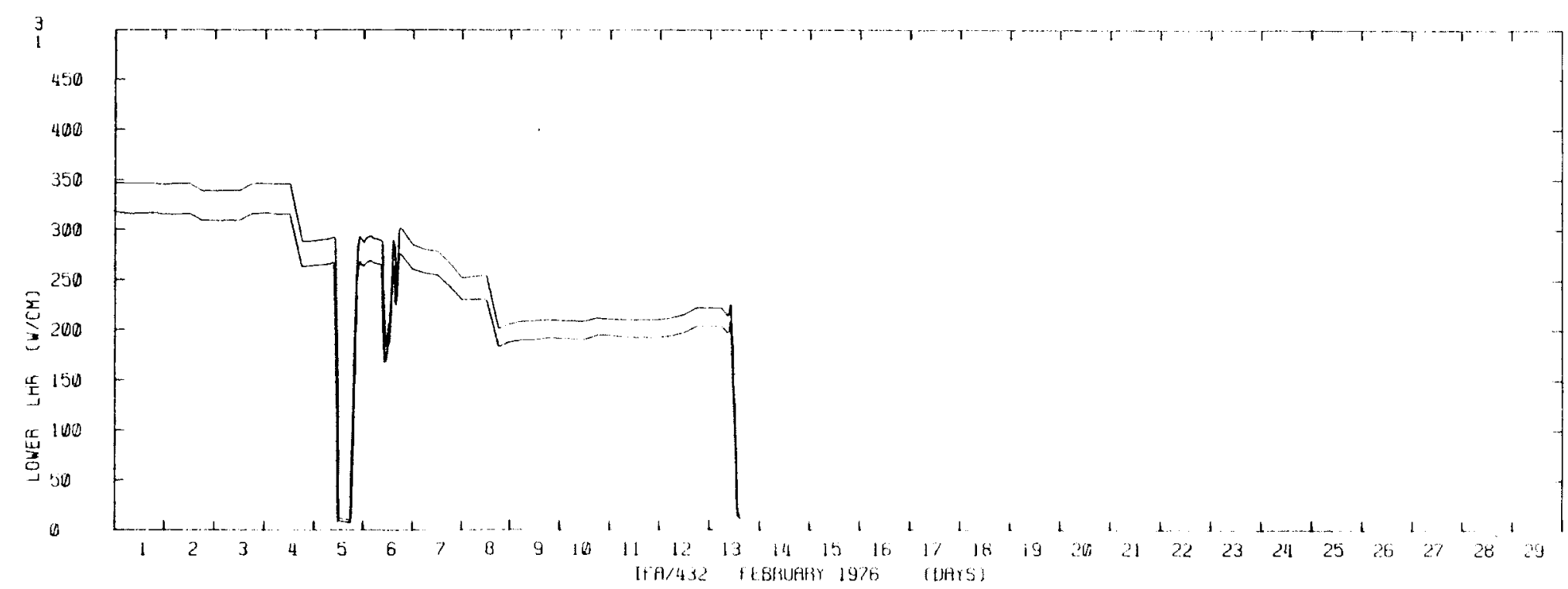

FIGURE 3.14. Local Linear Heat Ratings at Lower Thermocouple Locations for Rods 1,3 of IFA 432 - February 1976 


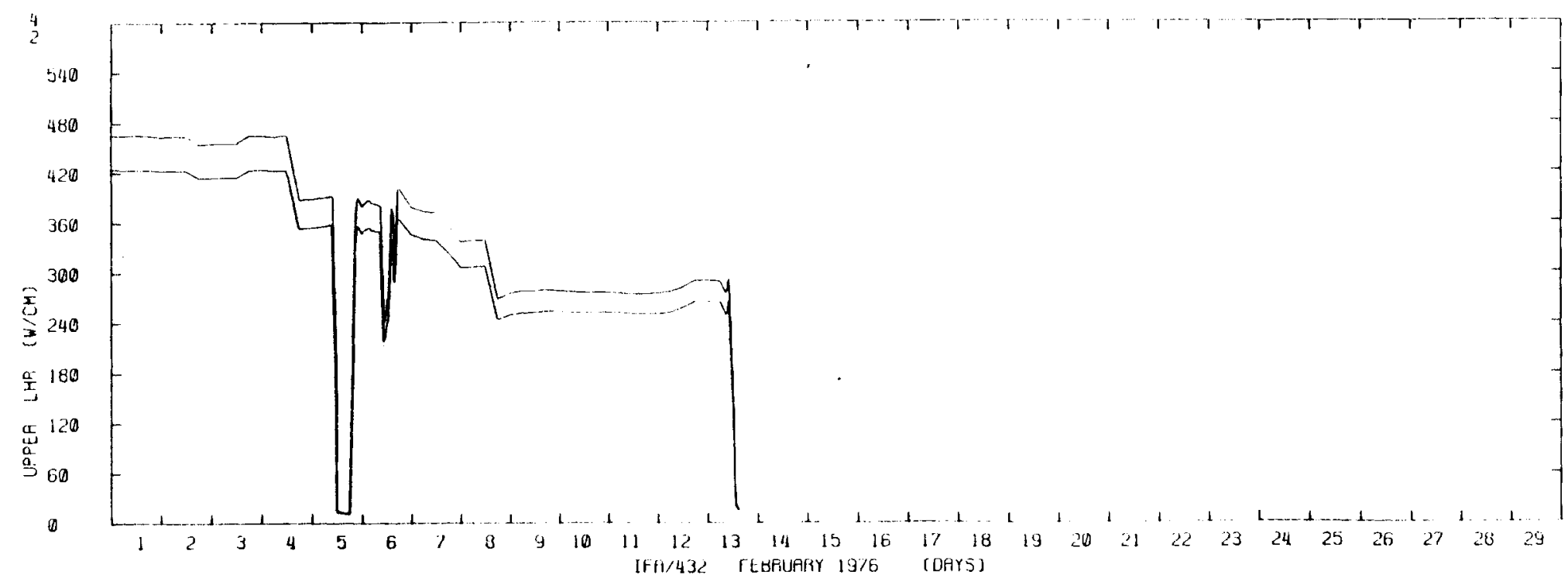

$\frac{w}{1}$

FIGURE 3.15. Local Linear Heat Ratings at Upper Thermocouple Locations for Rods 2,4 of IFA 432 - February 1976

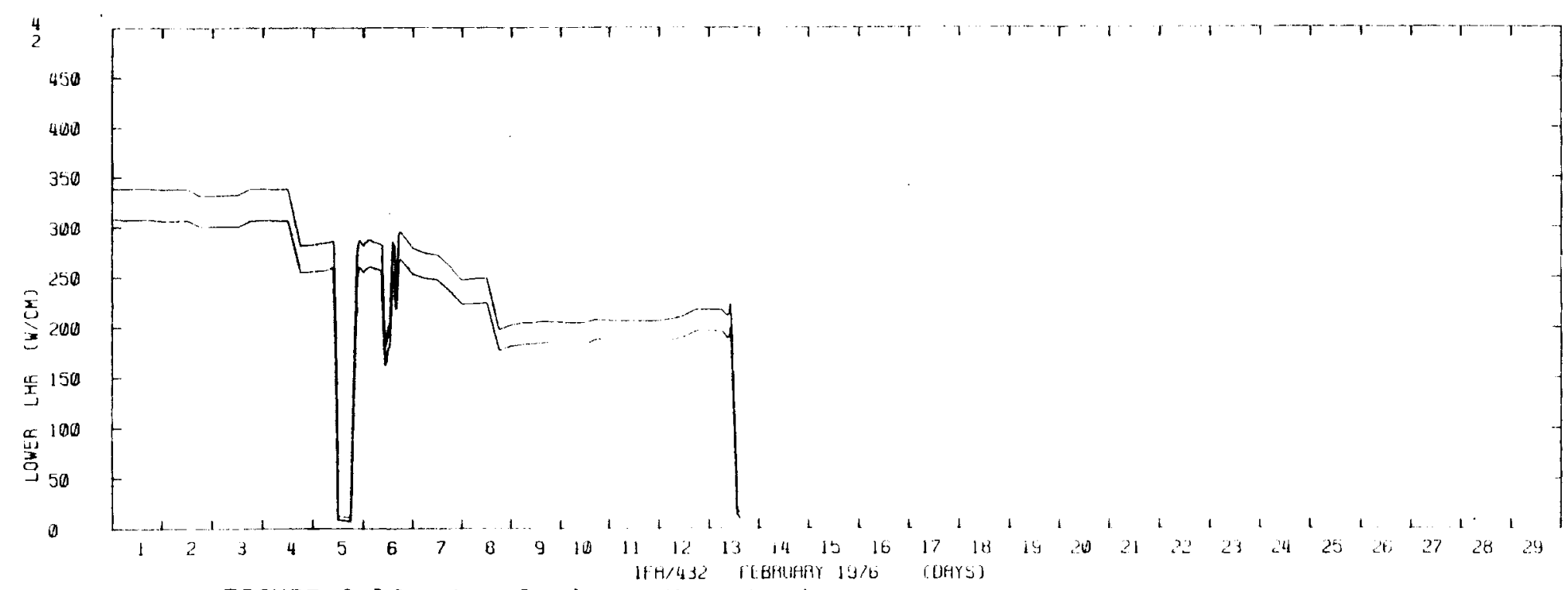

FIGURE 3.16. Local Linear Heat Ratings at Lower Thermocouple Locations for Rods 2,4 of IFA 432 - February 1976 


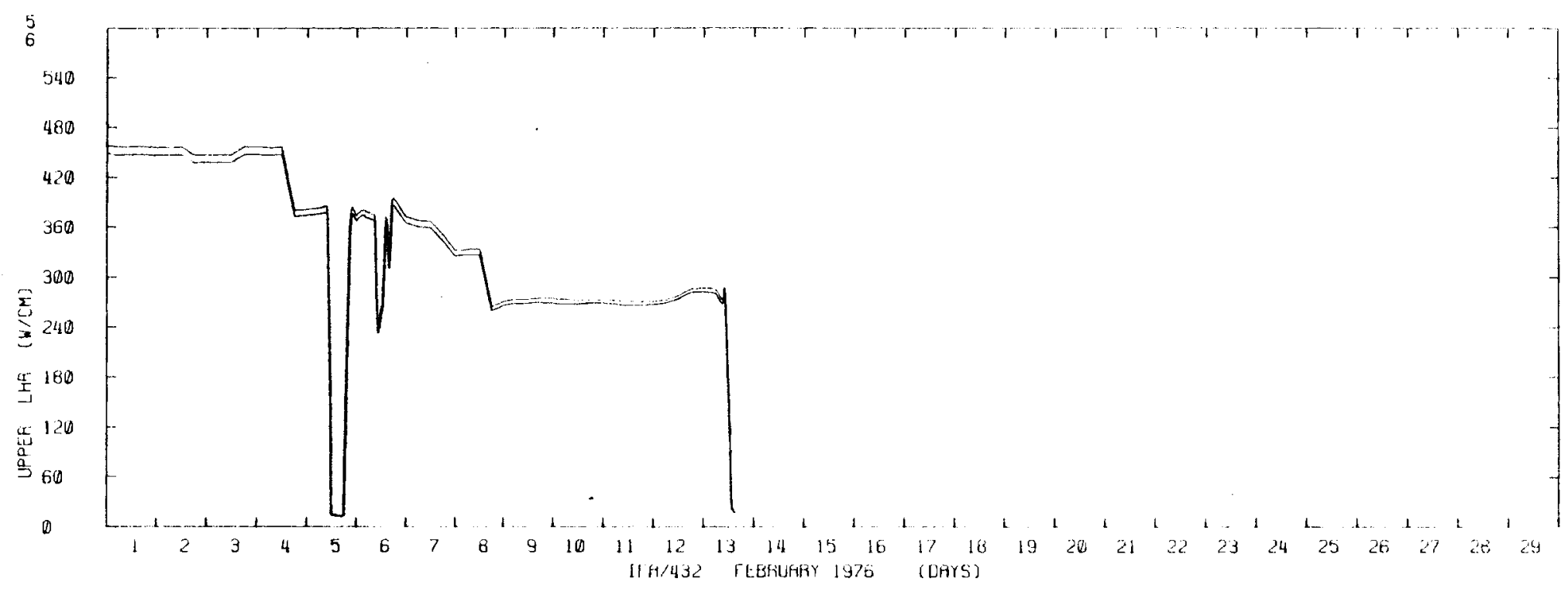

FIGURE 3.17. Local Linear Heat Ratings at Upper Thermocouple Locations for Rods 5,6 of IFA 432 - February 1976

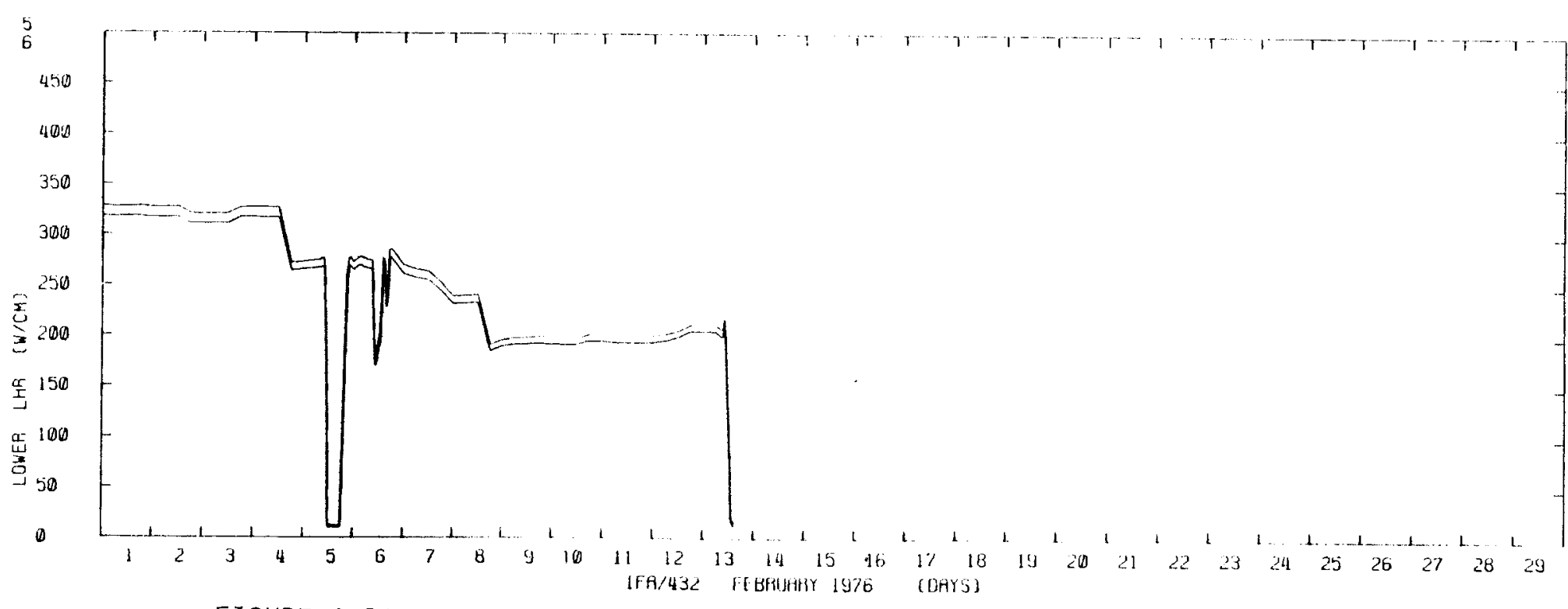

FIGURE 3.18. Local Linear Heat Ratings at Lower Thermocouple Locations for Rods 5,6 of IFA 432 - February 1976 
$\stackrel{\omega}{\omega}$

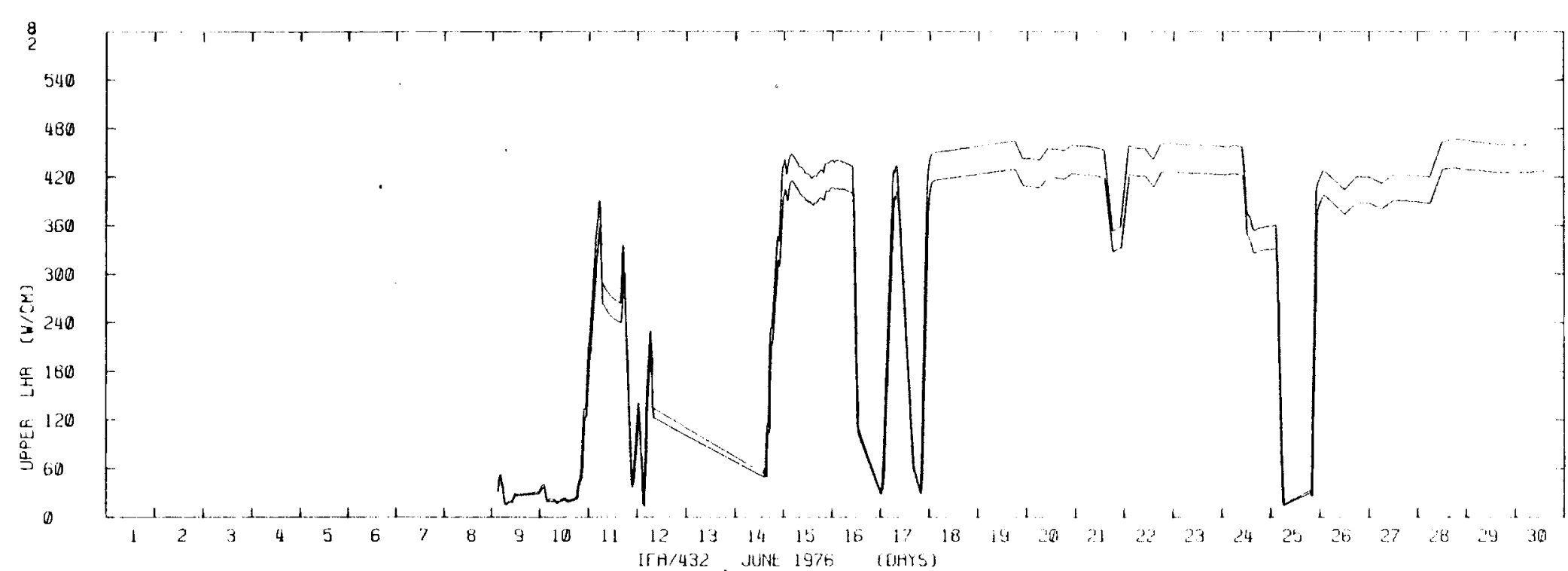

FIGURE 3.21. Local Linear Heat Ratings at Upper Thermocouple Locations for Rods 2,8 of IFA 432 - June 1976

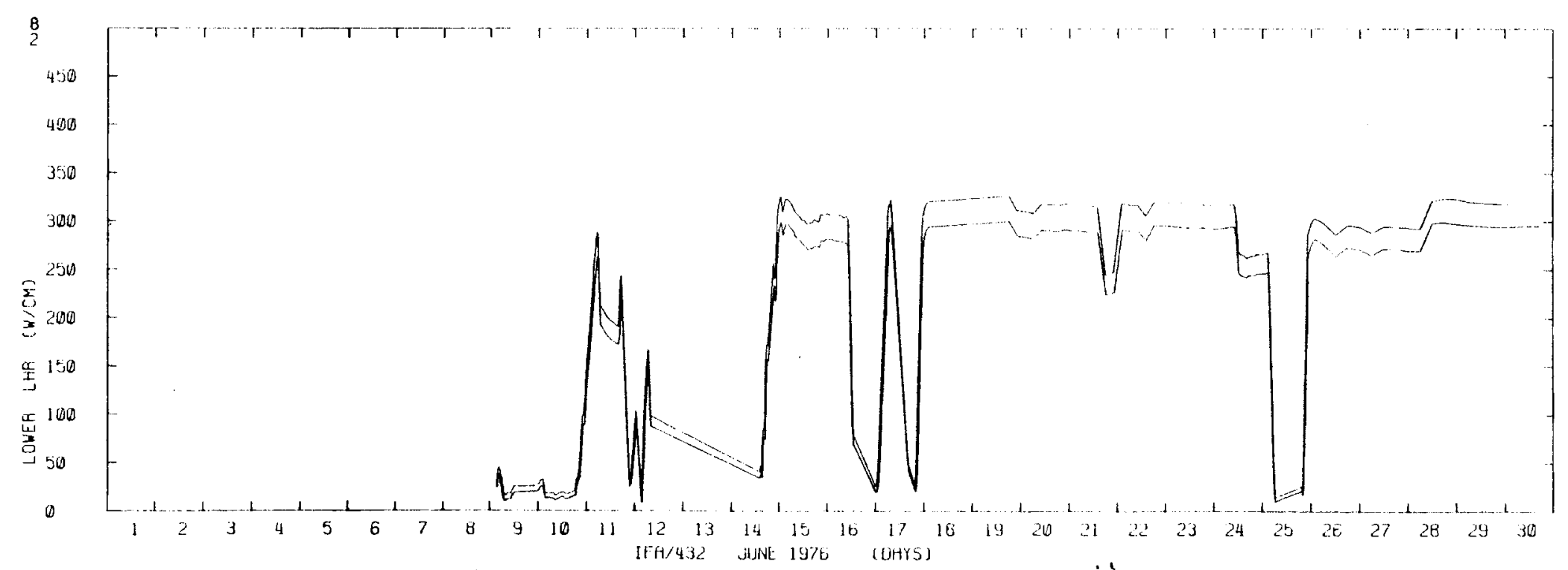

FIGURE 3.22. Local Linear Heat Ratings at Lower Thermocouple Locations for Rods 2,8 of IFA 432 - June 1976 


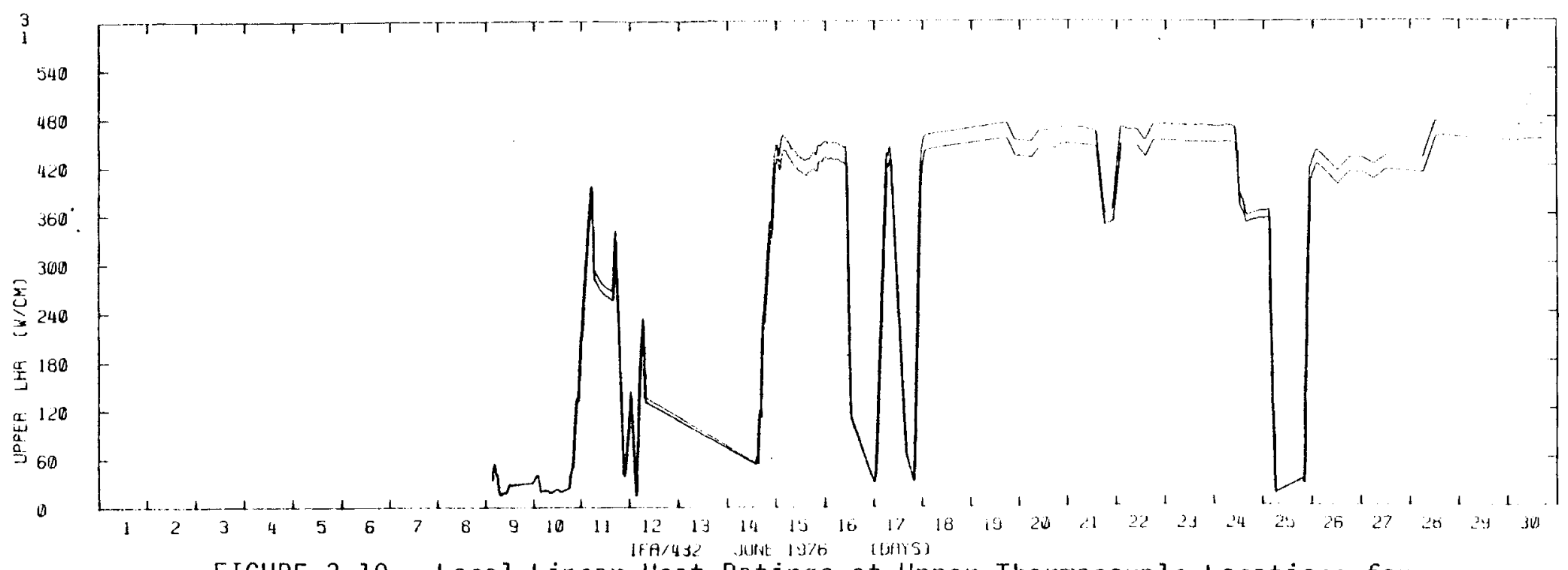

$\frac{w}{\sim}$

FIGURE 3.19. Local Linear Heat Ratings at Upper Thermocouple Locations for Rods 1,3 of IFA 432 - June 1976

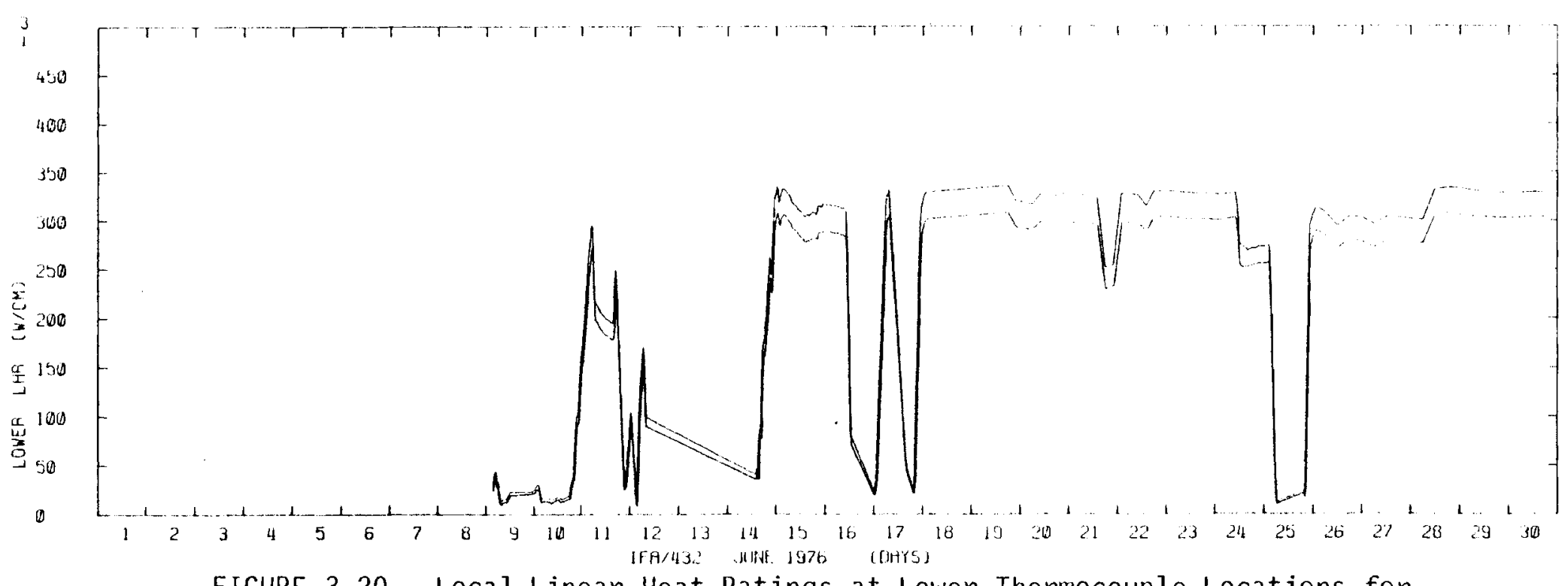

FIGURE 3.20. Local Linear Heat Ratings at Lower Thermocouple Locations for Rods 1,3 of IFA 432 - June 1976 


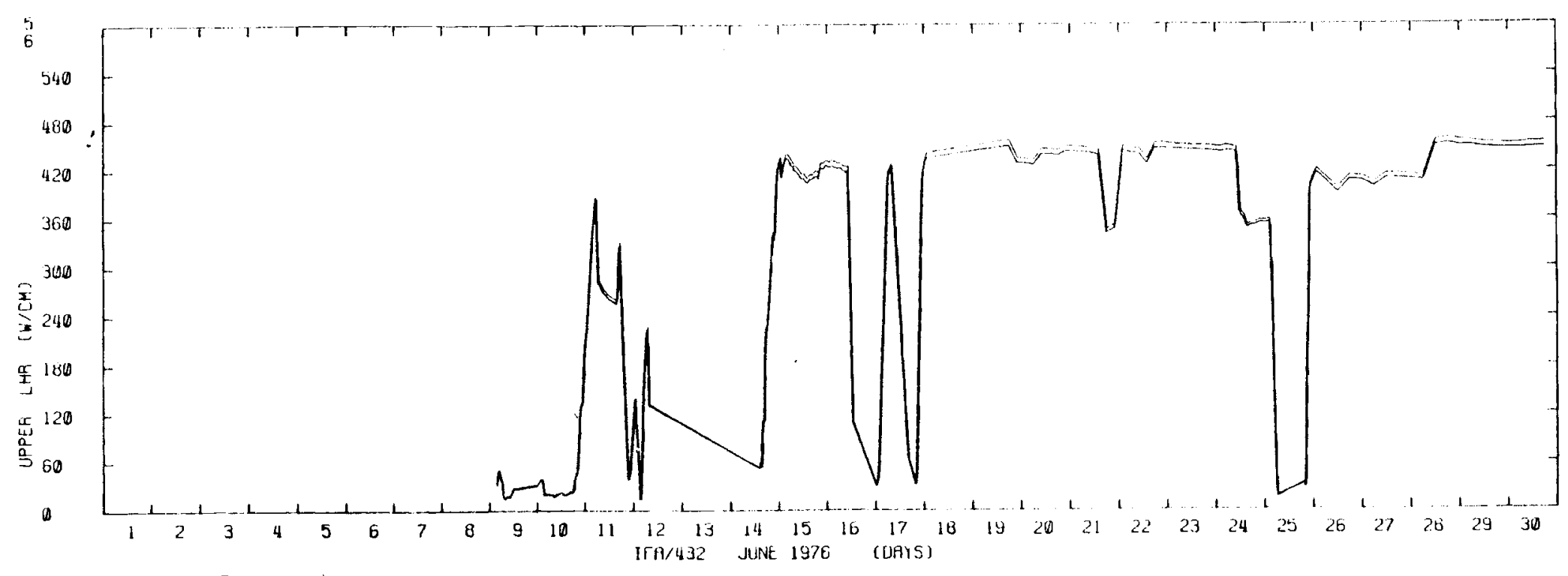

$\frac{\omega}{a}$

FIGURE 3.23. Local Linear Heat Ratings at Upper Thermocouple Locations for Rods 5,6 of IFA 432 - June 1976

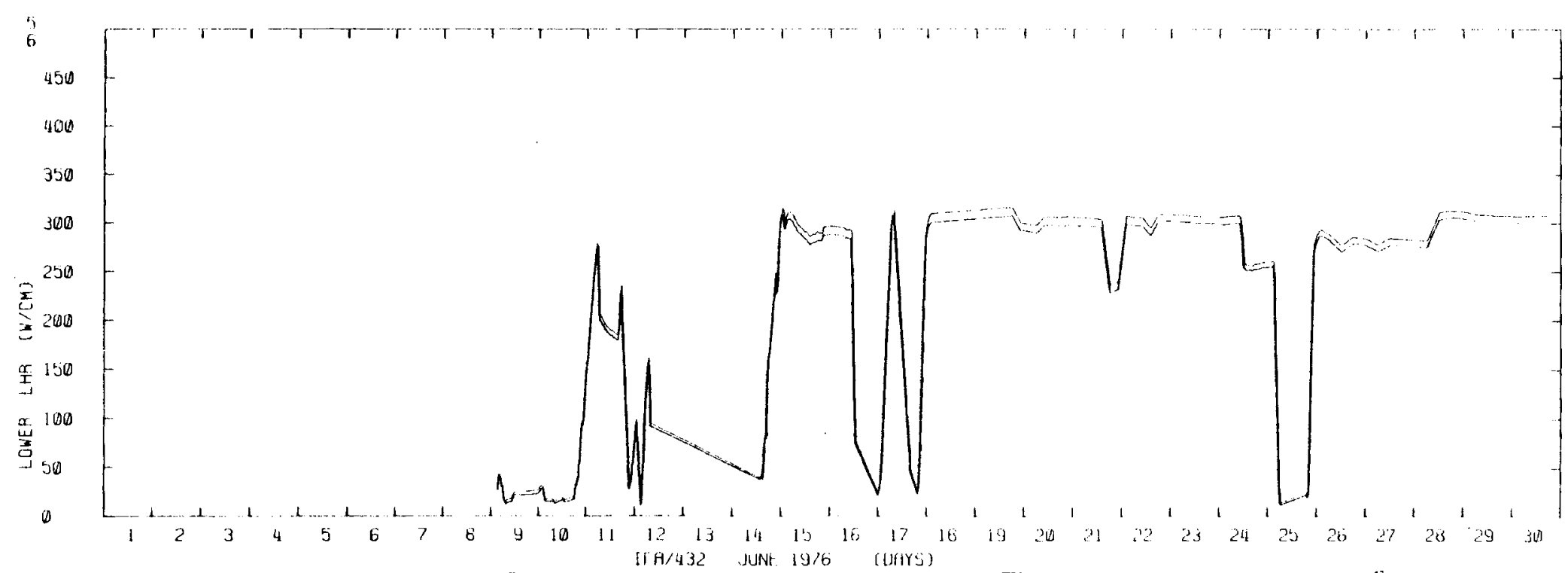

FIGURE 3.24. Local Linear Heat Ratings at Lower Thermocouple Locations for Rods 5,6 of IFA 432 - June 1976 


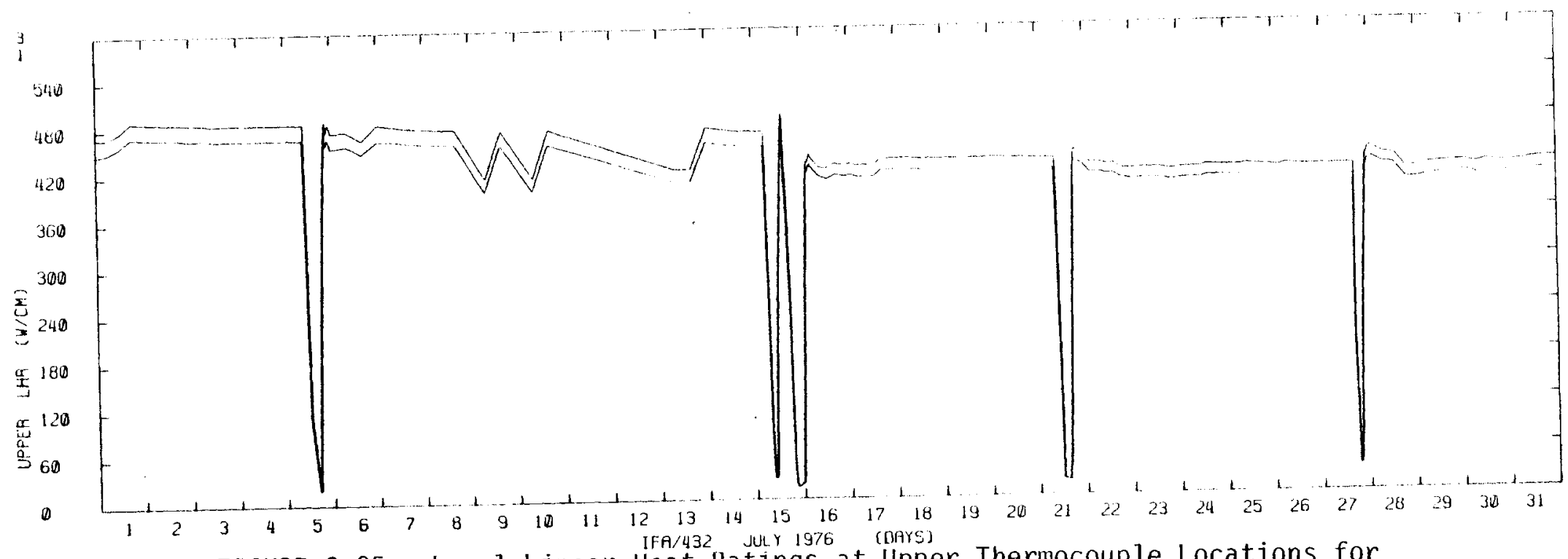

FIGURE 3.25. Local Linear Heat Ratings at Upper Thermocouple Locations for Rods 1,3 of IFA 432 - July 1976

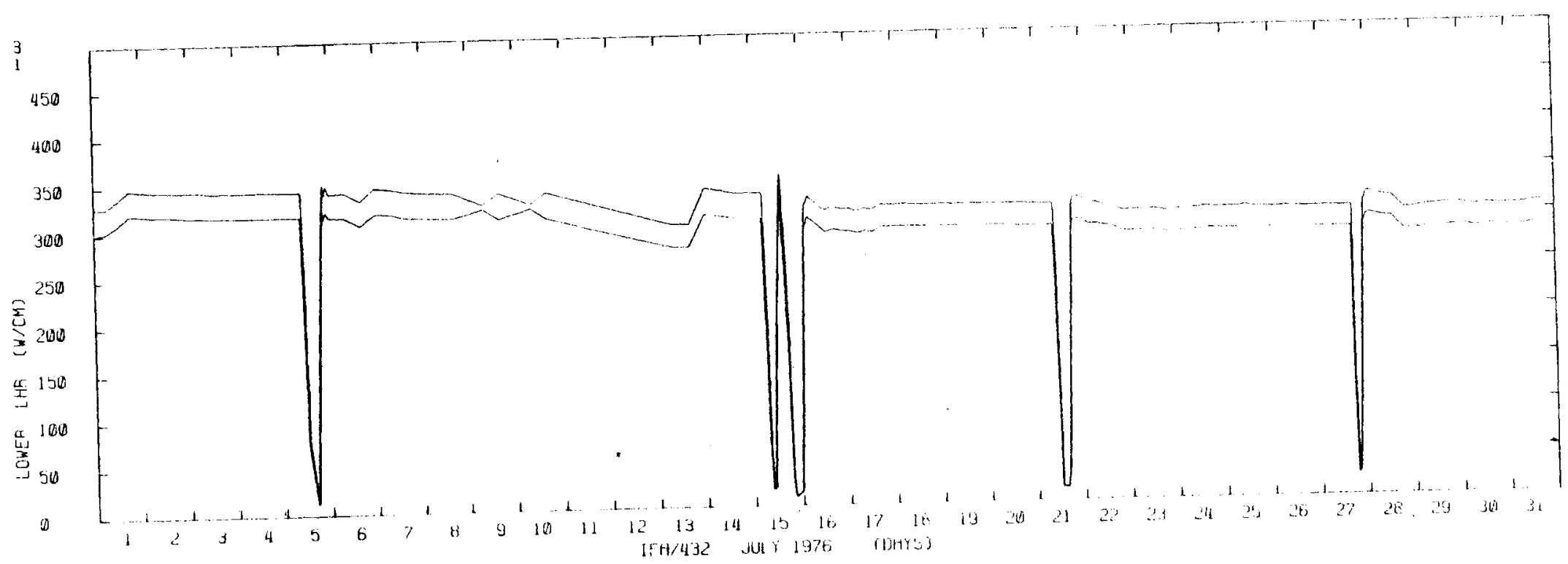

FIGURE 3.26. Local Linear Heat Ratings at Lower Thermocouple Locations for Rods 1,3 of IFA 432 - July 1976 


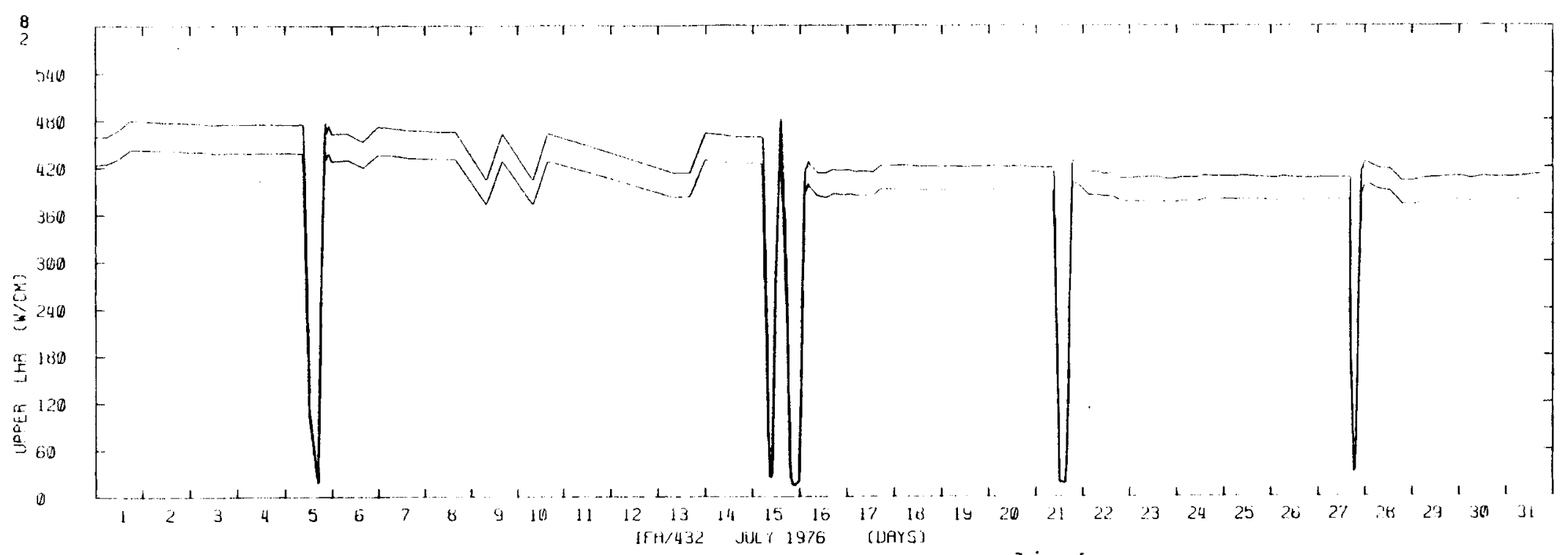

$\stackrel{\omega}{\frac{1}{\sigma}}$

FIGURE 3.27. Local Linear Heat Ratings at Upper Thermocouple Locations for Rods 2,8 of IFA 432 - July 1976

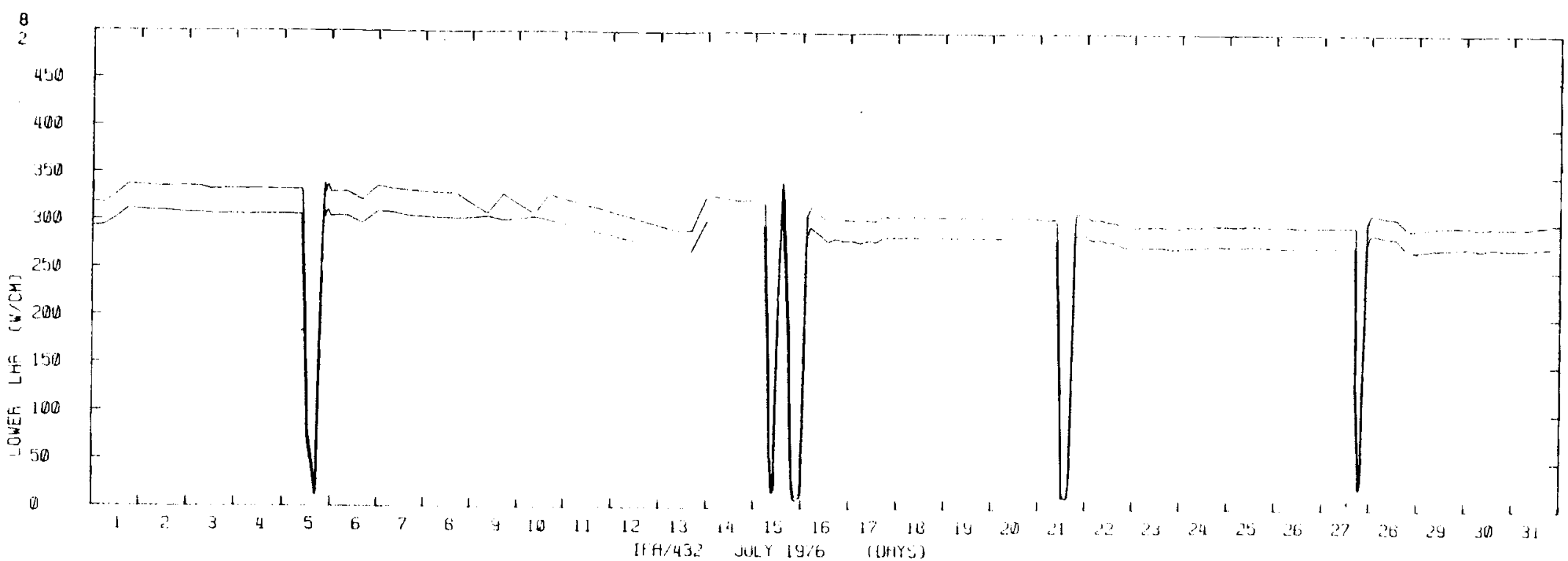

FIGURE 3.28. Local Linear Heat Ratings at Lower Therimocouple Locations for Rods 2,8 of IFA $432^{\circ}$ - July 1976 


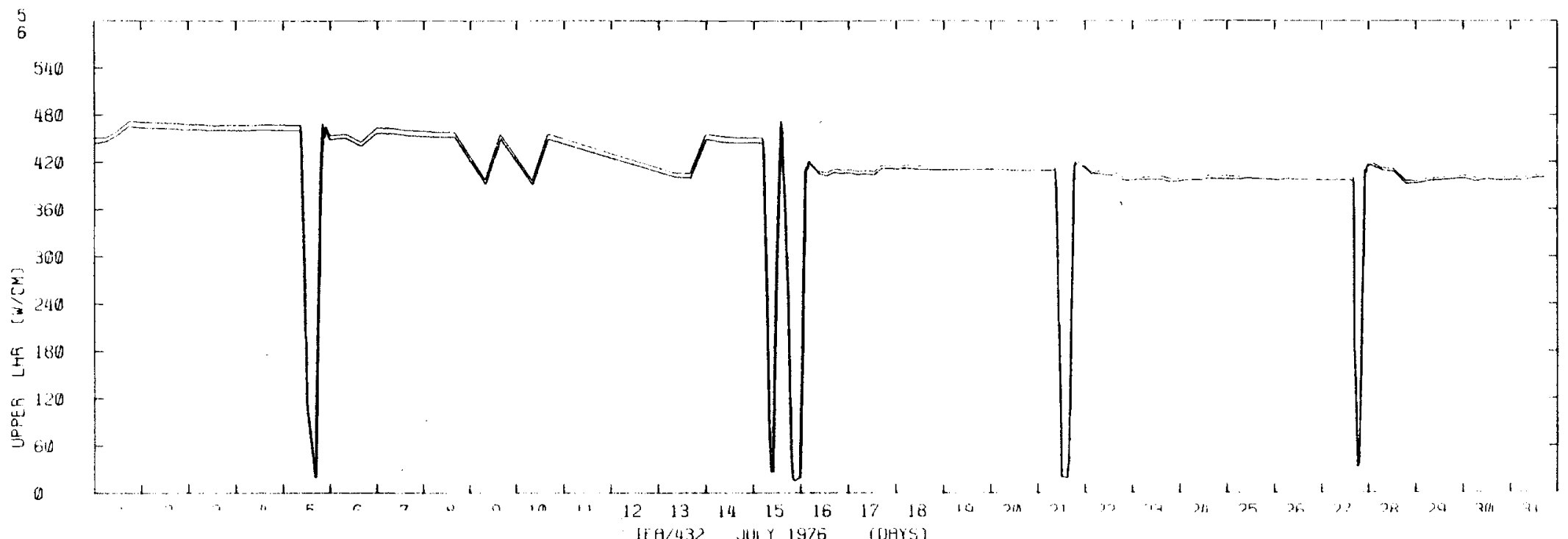

$\stackrel{\dot{1}}{\vec{v}}$

FIGURE 3.29. Local Linear Heat Ratings at Upper Thermocouple Locations for Rods 5,6 of IFA 432 - July 1976

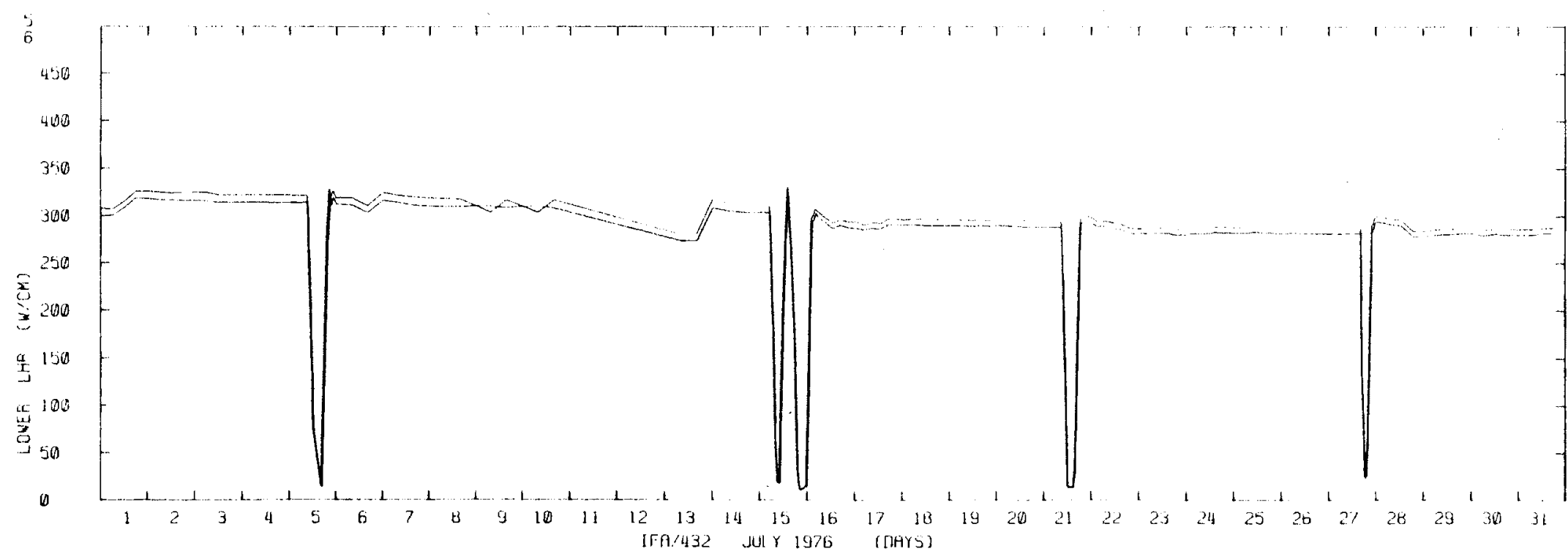

FIGURE 3.30. Local Linear Heat Ratings at Lower Thermocouple Locations for Rods 5,6 of IFA 432 - July 1976 


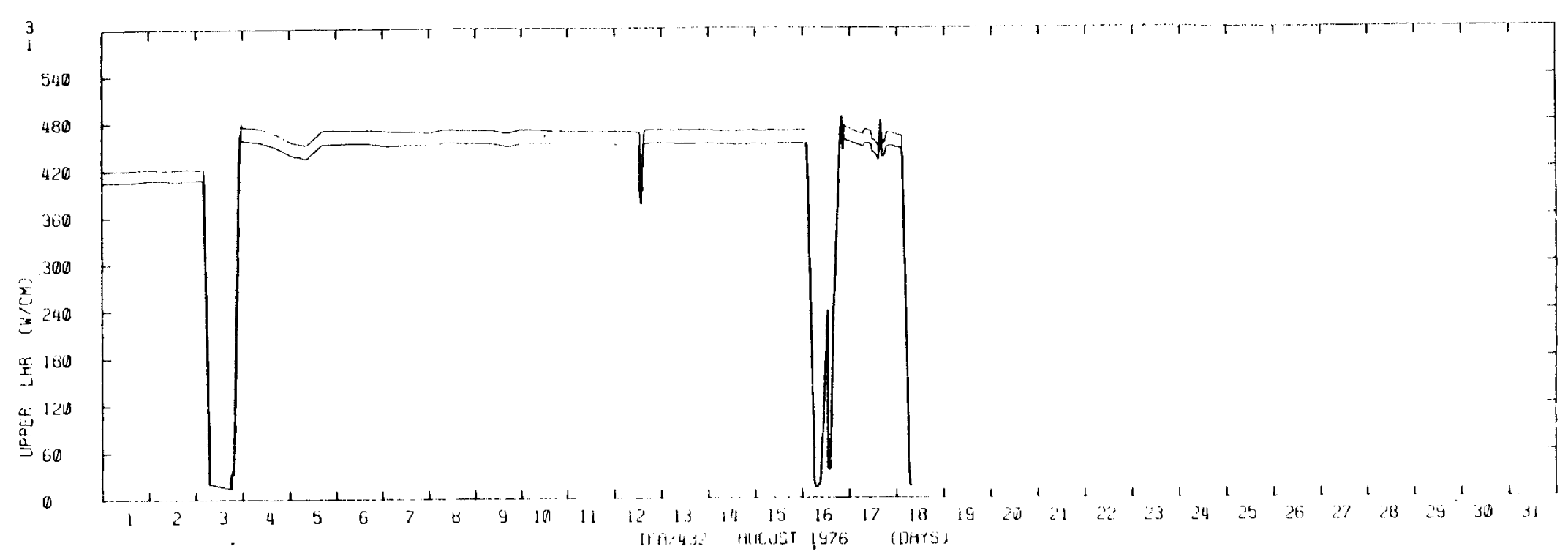

$\stackrel{\omega}{\dot{\omega}}$

FIGURE 3.31. Local Linear Heat Ratings at Upper Thermocouple Locations for Rods 1,3 of IFA 432 - August 1976

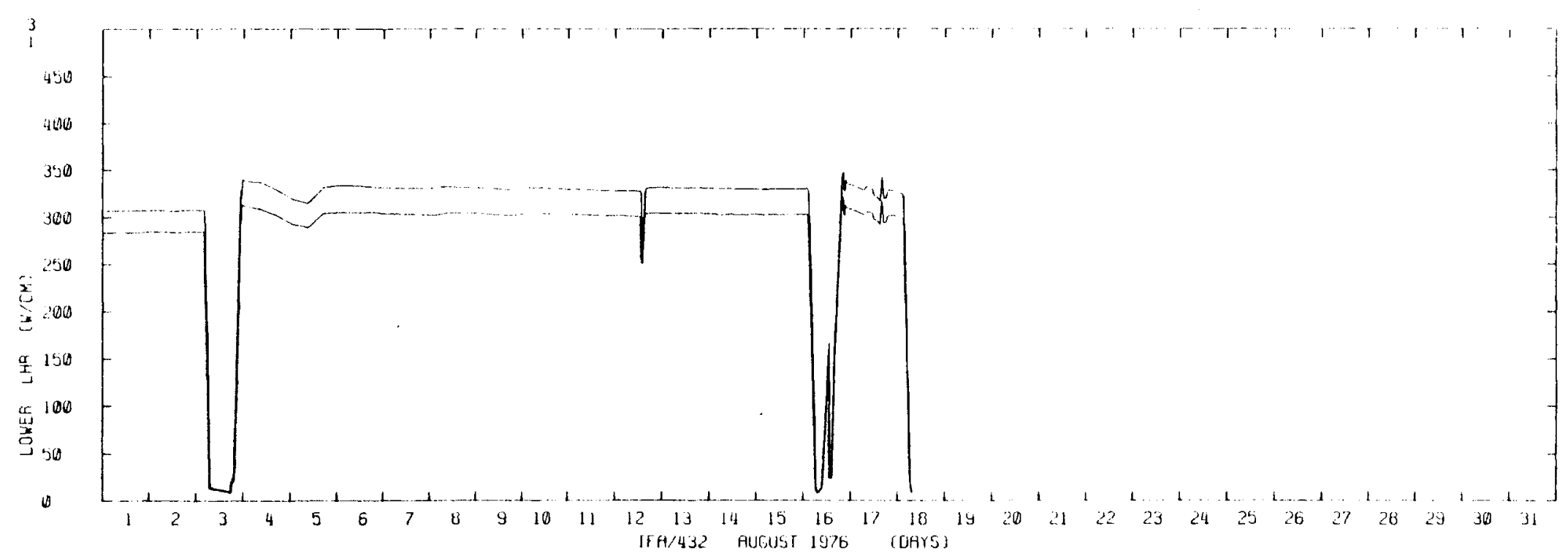

FIGURE 3.32. Local Linear Heat Ratings at Lower Thermocouple Locations for Rods 1,3 of IFA 432 - August 1976 


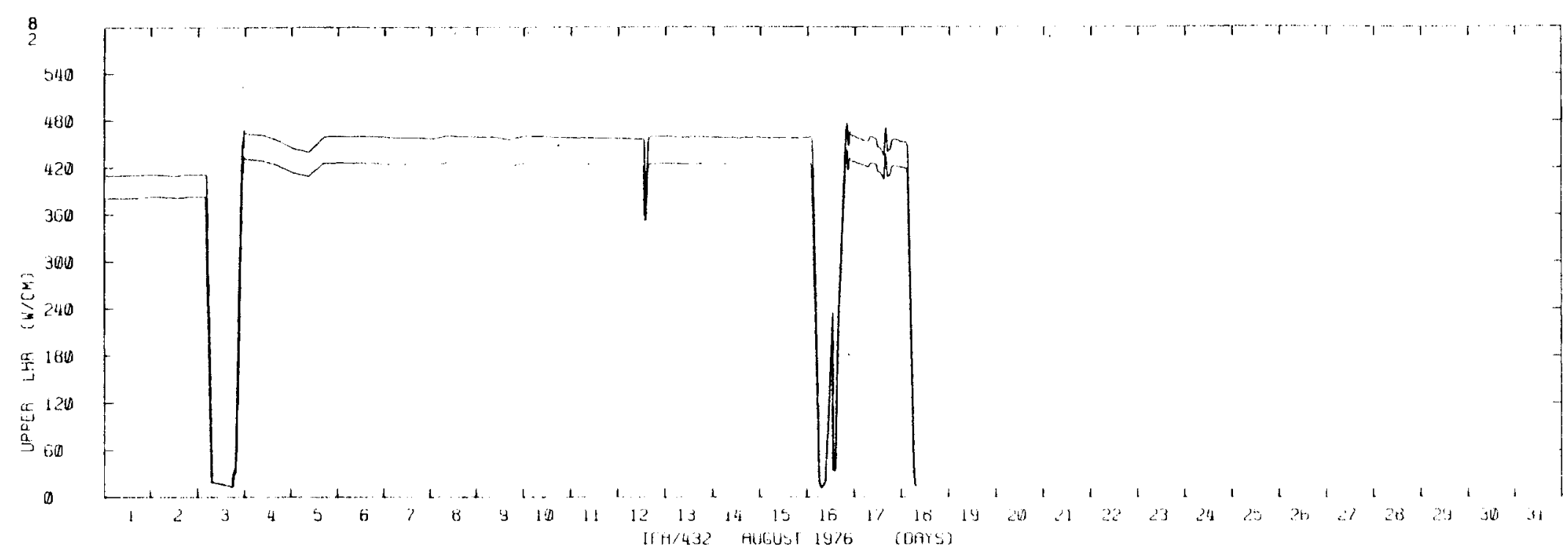

$\frac{\omega}{6}$

FIGURE 3.33. Local Linear Heat Ratings at Upper Thermocouple Locations for Rods 2,8 of IFA 432 - August 1976

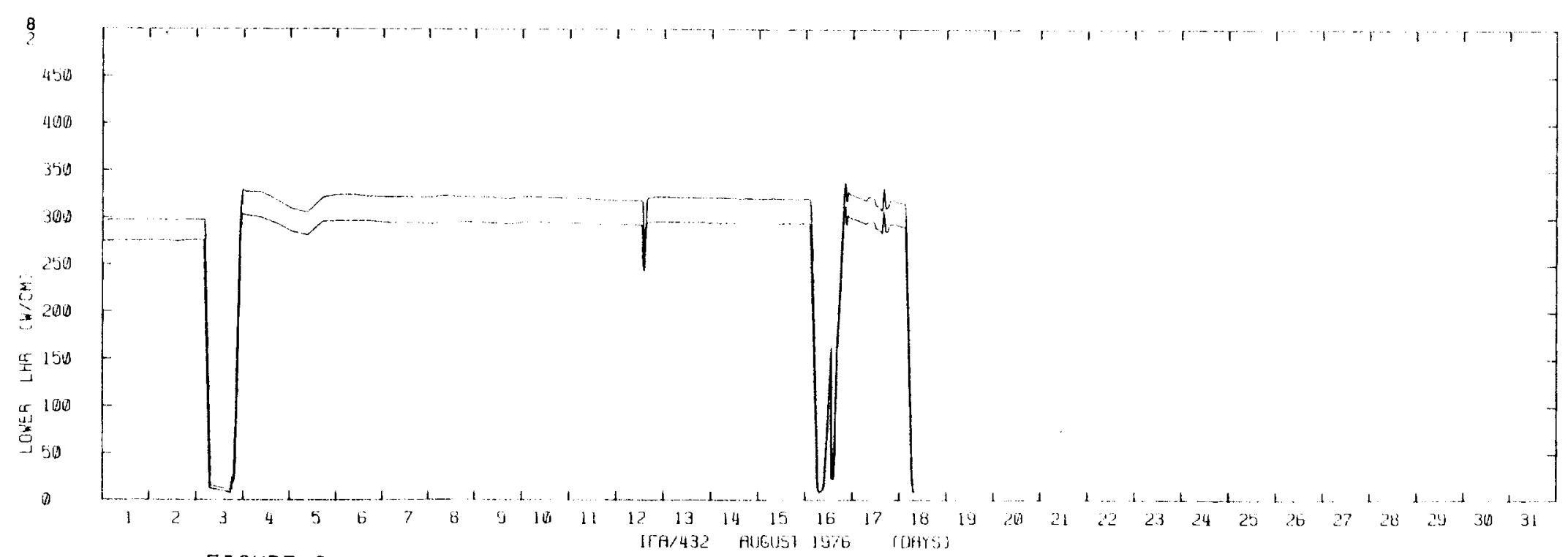

FIGURE 3.34. Local Linear Heat Ratings at Lower Thermocouple Locations for Rods 2,8 of IFA 432 - August 1976 


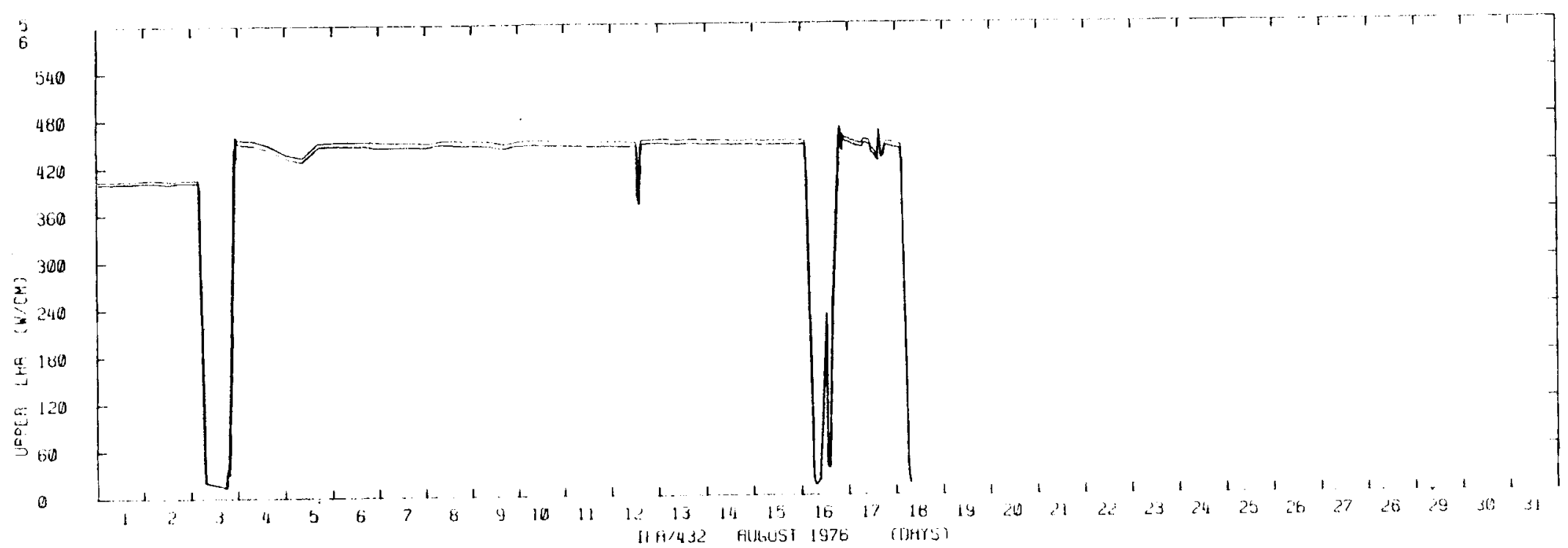

FIGURE 3.35. Local Linear Heat Ratings at Upper Thermocouple Locations for Rods 5,6 of IFA 432 - August 1976

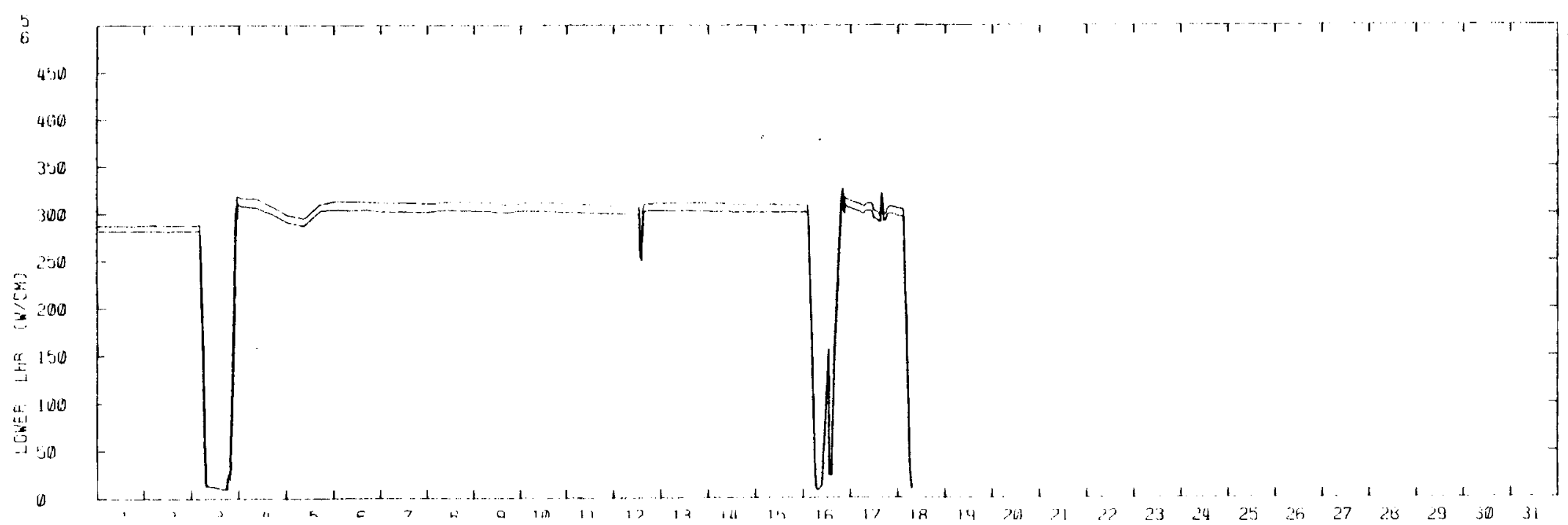

FIGURE 3.36. Local Linear Heat Ratings at Lower Thermocouple Locations for Rods 5,6 of IFA 432 - August 1976 


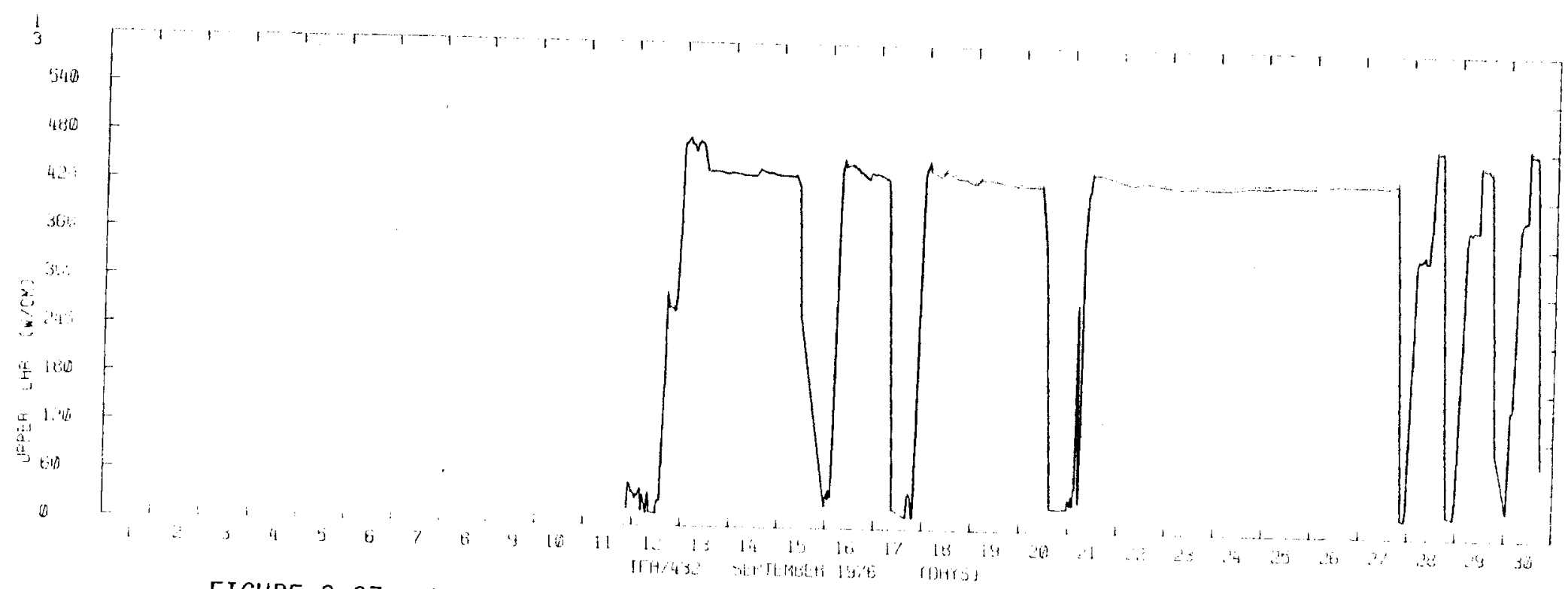

FIGURE 3.37. Local Linear Heat Ratings at Upper Thermocouple Locations for Rods 1,3 of IFA 432 - September 1976

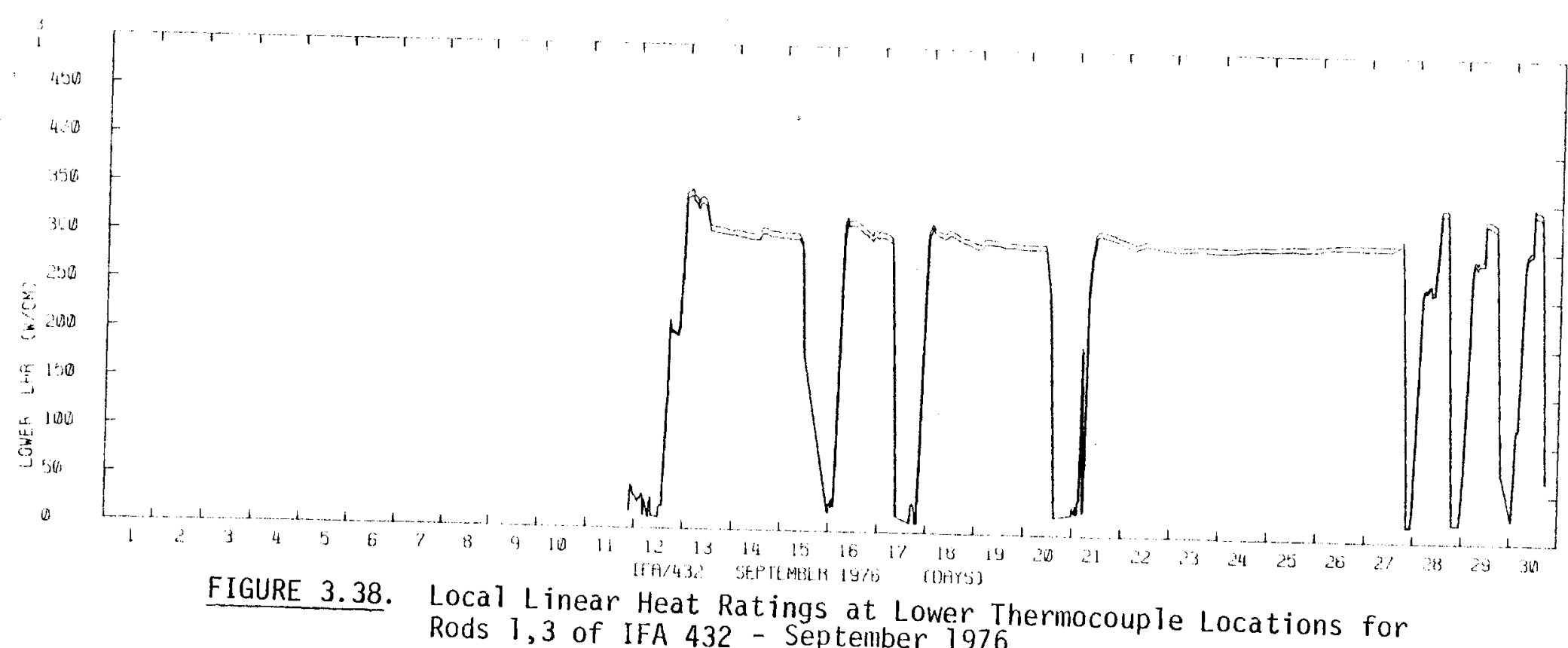




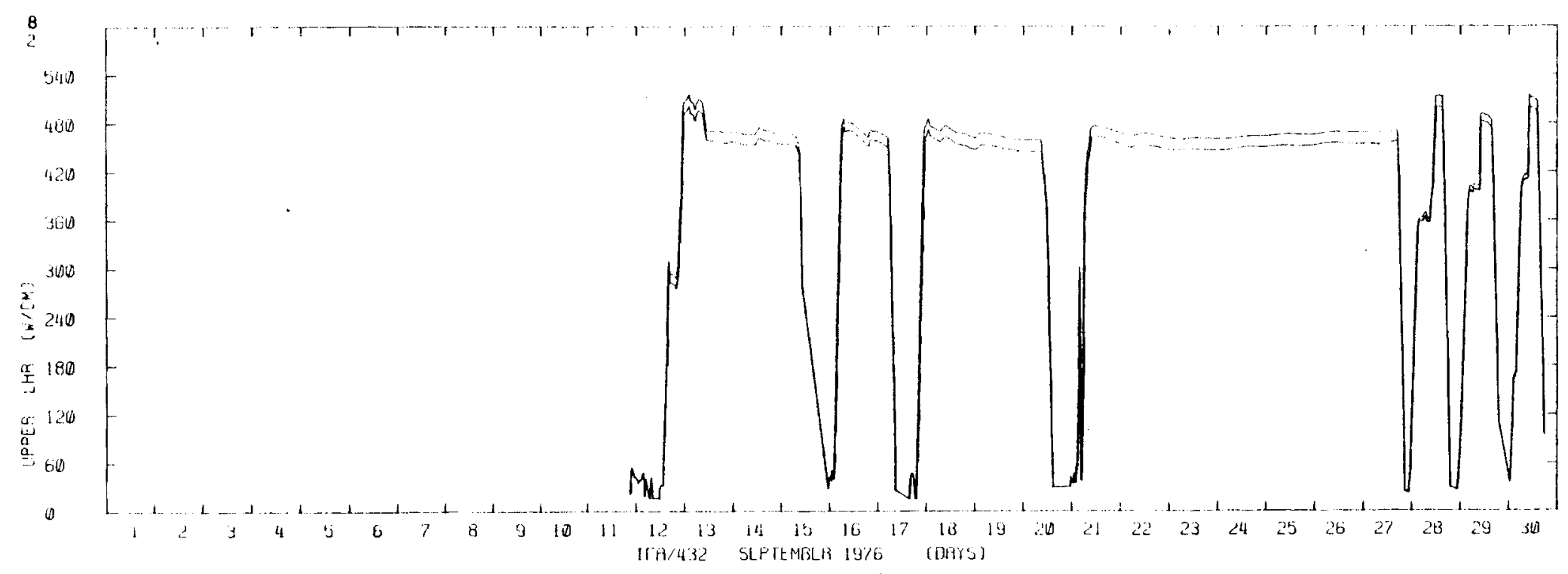

岕

FIGURE 3.39. Local Linear Heat Ratings at Upper Thermocouple Locations for Rods 2,8 of IFA 432 - September 1976

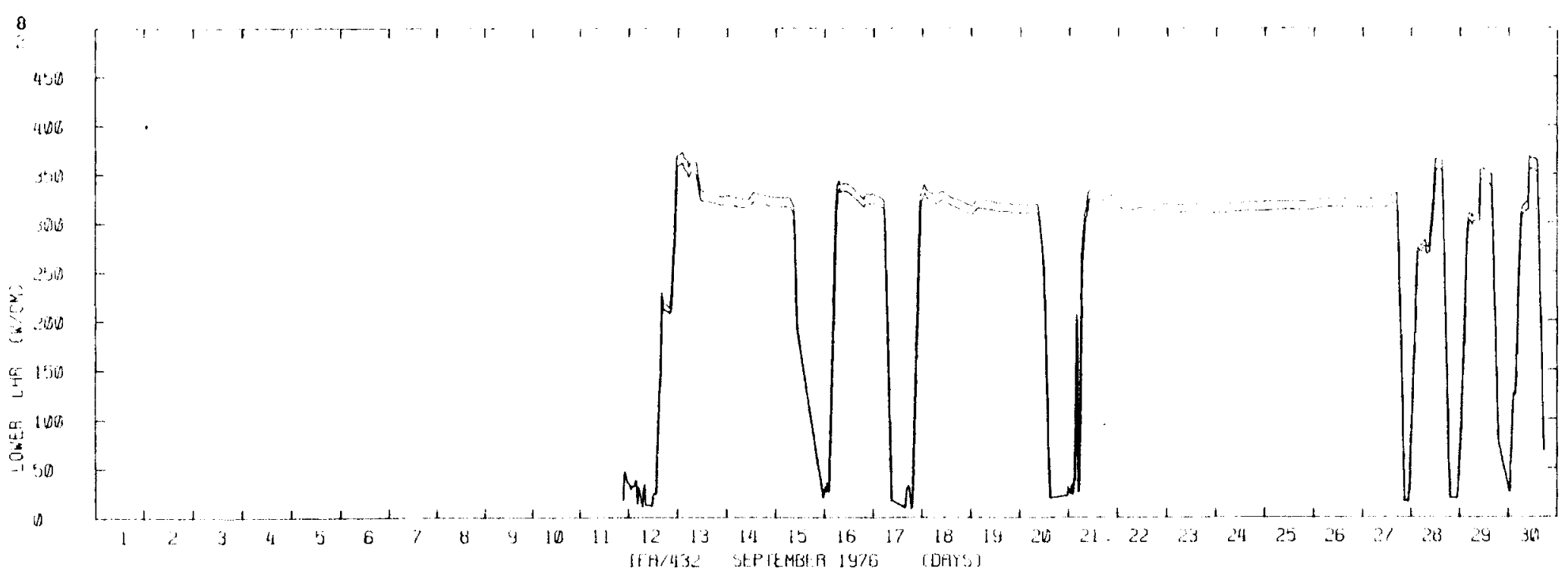

FIGURE 3.40. Local Linear Heat Ratings at Lower Thermocouple Locations for Rods 2,8 of IFA 432 - Septenber 1976 


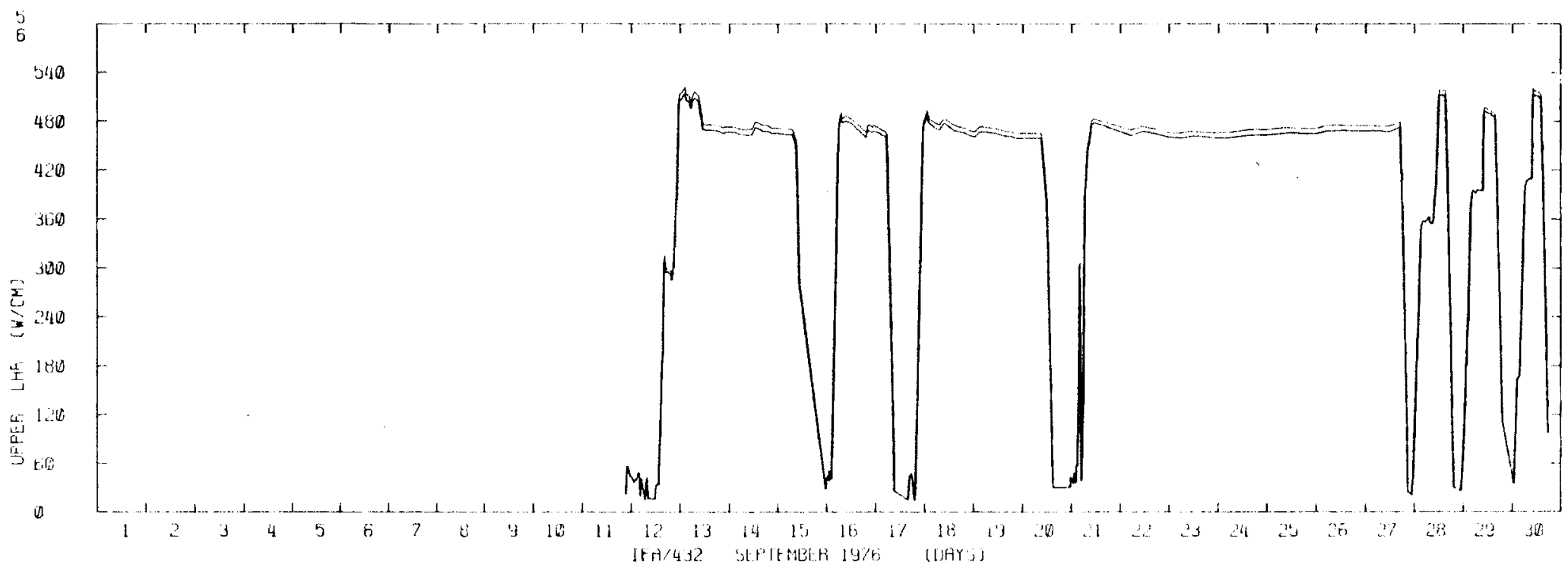

FIGURE 3.41. Local Linear Heat Ratings at Upper Thermocouple Locations for Rods 5,6 of IFA 432 . September 1976

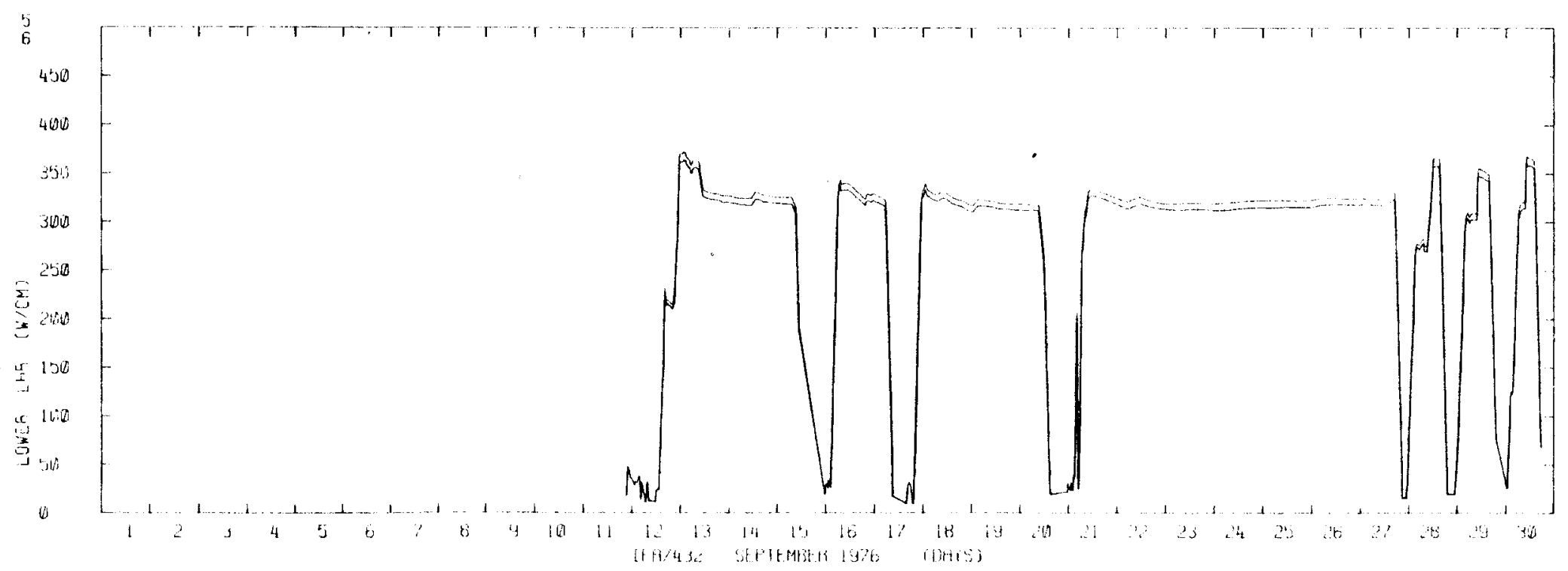

FIGURE 3.42. Local Linear Heat Ratings at Lower Thermocouple Locations for Rods 5,6 of IFA 432 - September 1976 


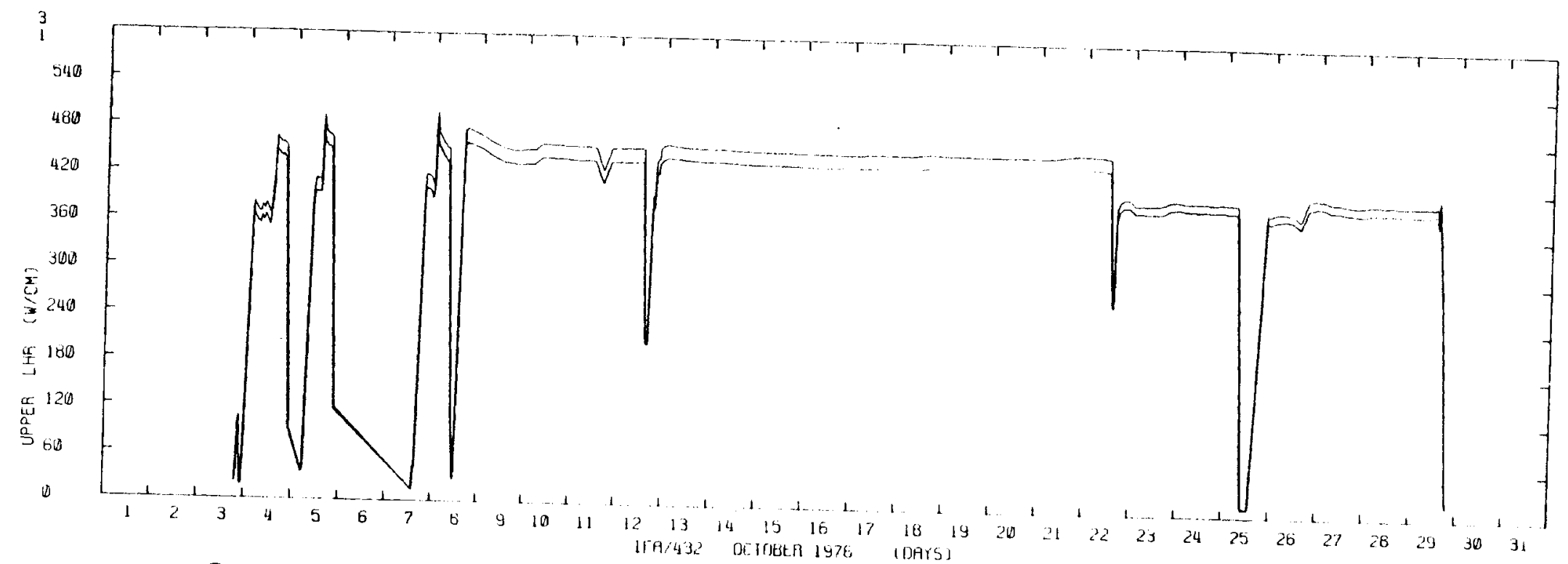

$\stackrel{\omega}{\stackrel{\omega}{\infty}}$

FIGURE 3.43. Local Linear Heat Ratings at Upper Thermocouple Locations for Rods 1,3 of IFA 432 - October 1976

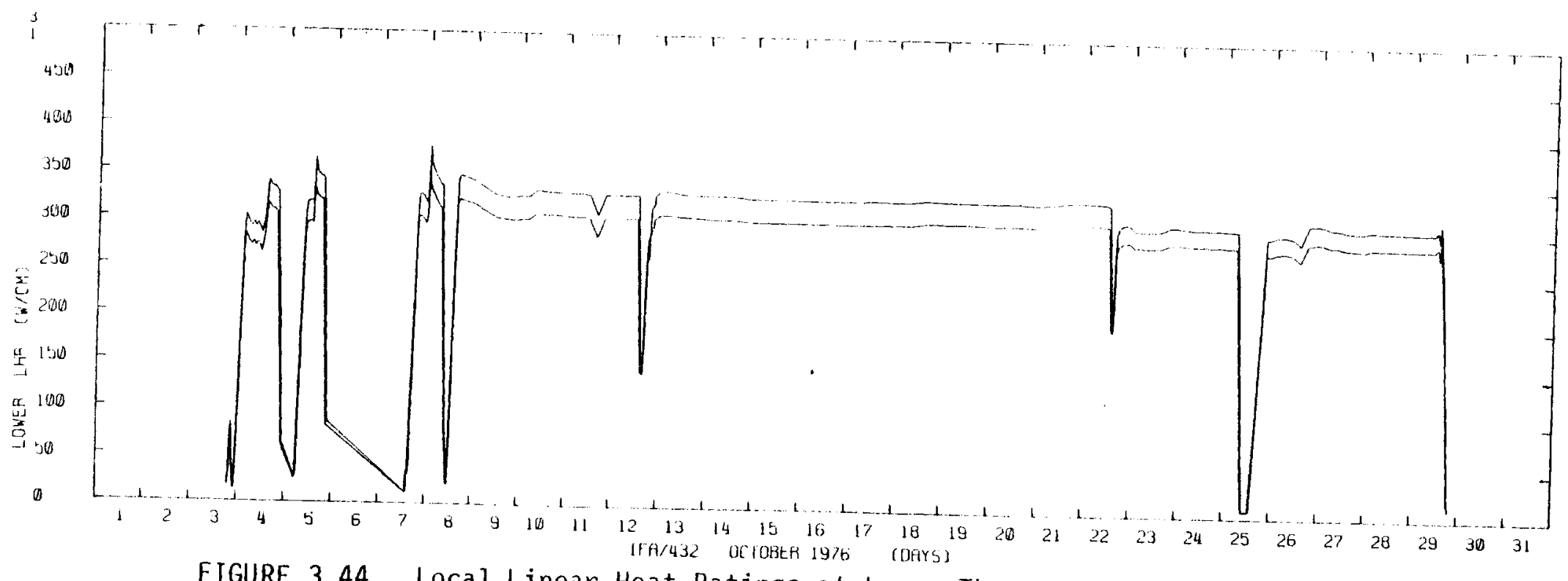

FIGURE 3.44. Local Linear Heat Ratings at Lower Thermocouple Locations for Rods 1,3 of IFA 432 - October 1976 


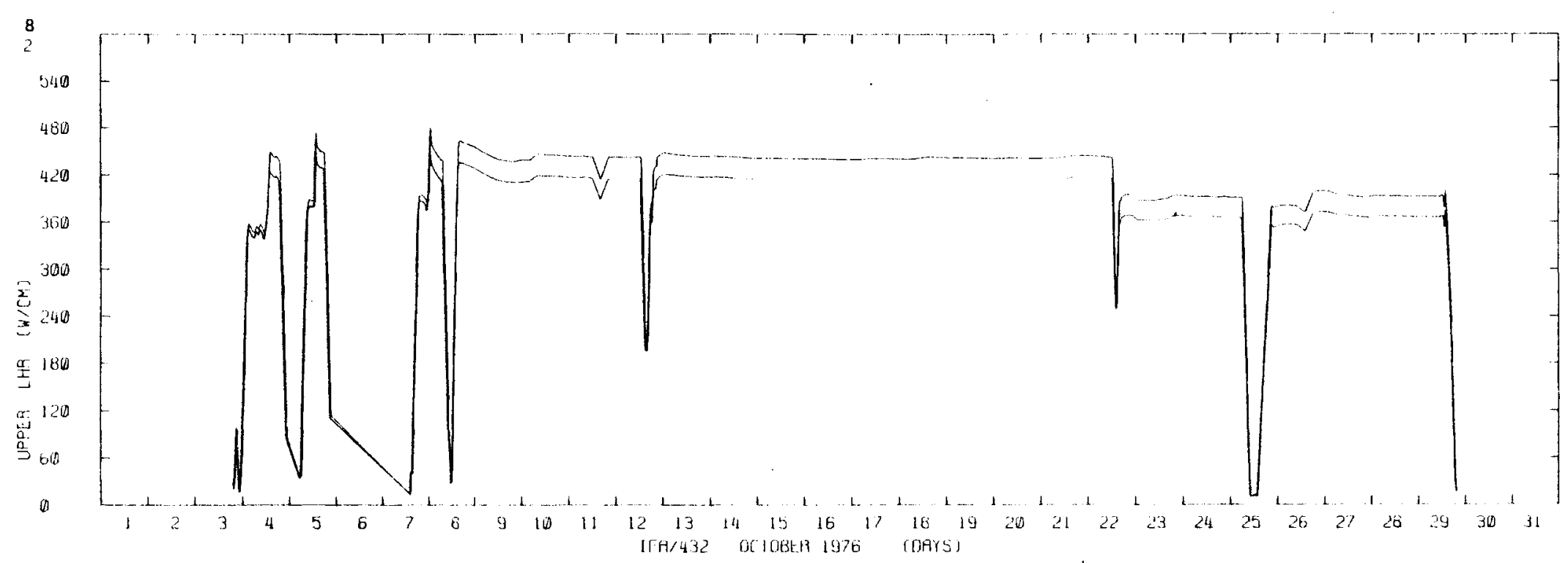

$\stackrel{w}{1}$

FIGURE 3.45. Local Linear Heat Ratings at Upper Thermocouple Locations for Rods 2,8 of IFA 432 - October 1976

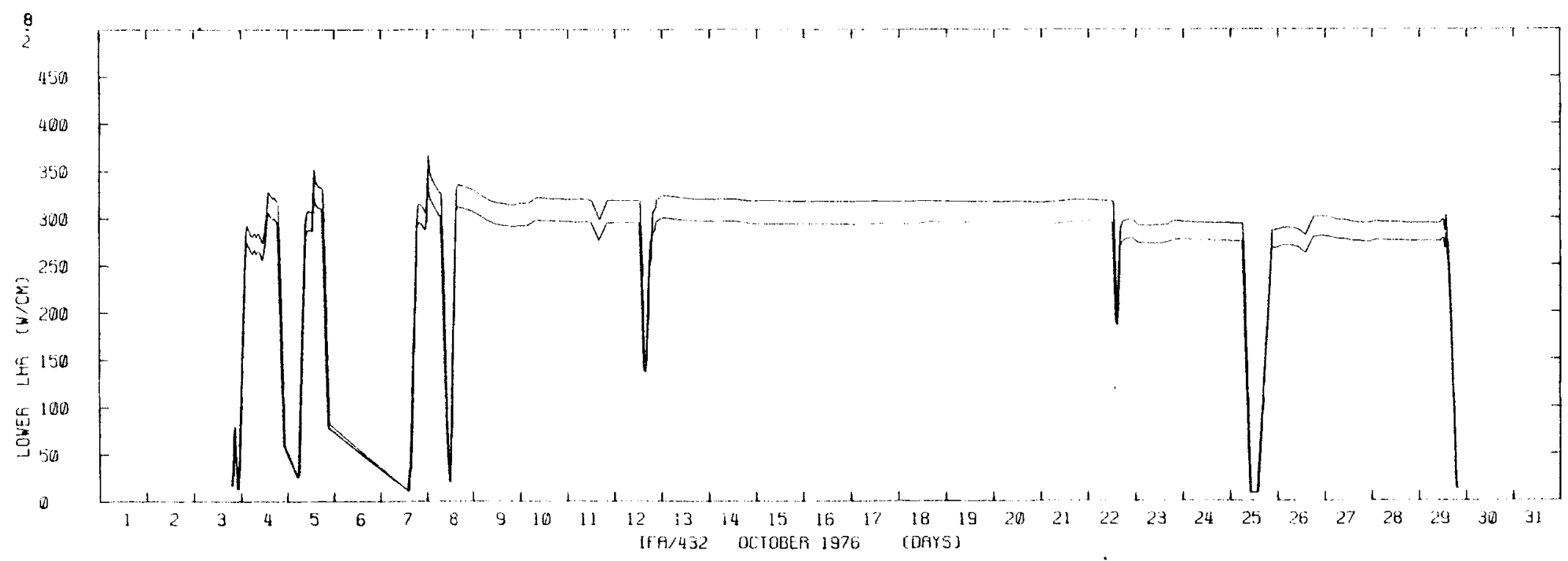

FIGURE 3.46. Local Linear Heat Ratings at Lower Thermocouple Locations for Rods 2,8 of IFA 432 - October 1976 


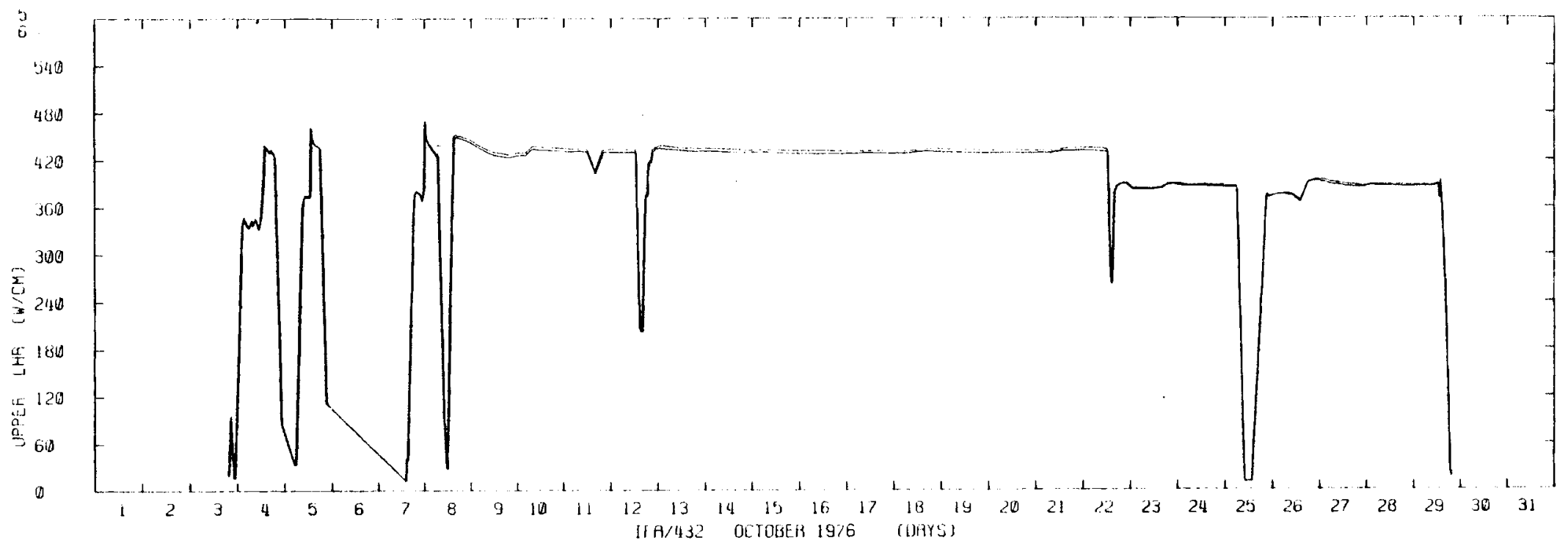

FIGURE 3.47. Local Linear Heat Ratings at Upper Thermocouple Locations for Rods 5,6 of IFA 432 - October 1976

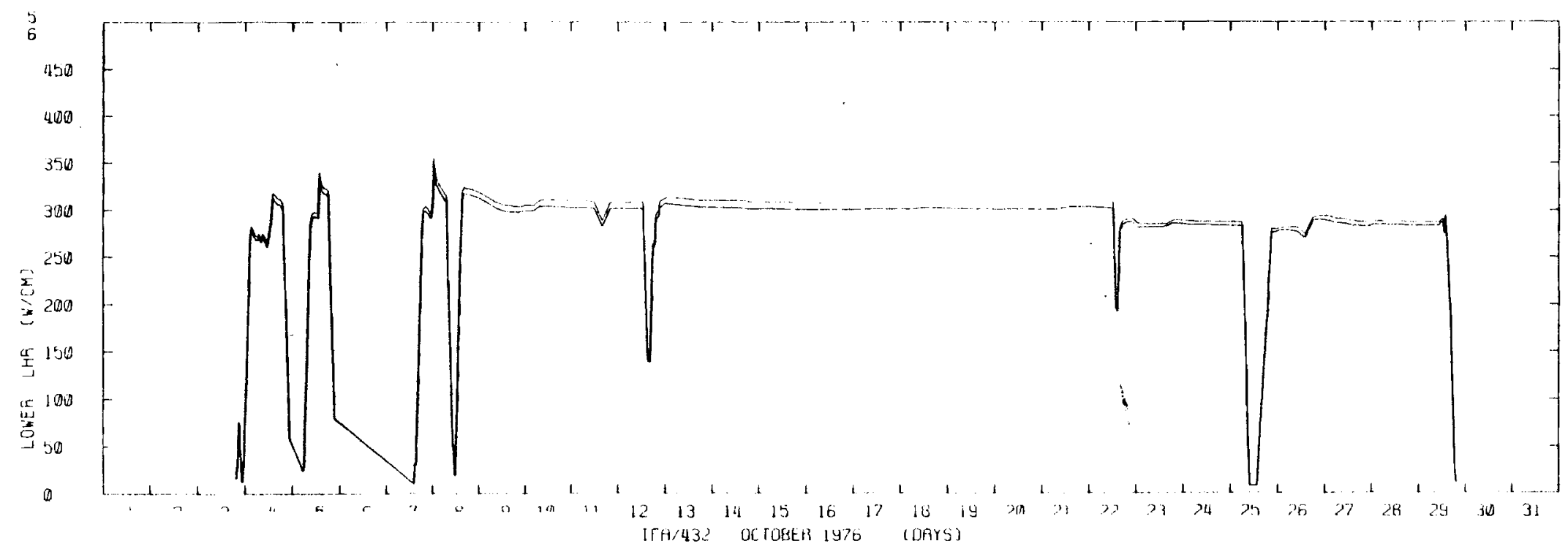

FIGURE 3.48. Local Linear Heat Ratings at Lower Thermocouple Locations for Rods 5,6 of IFA 432 - October 1976 


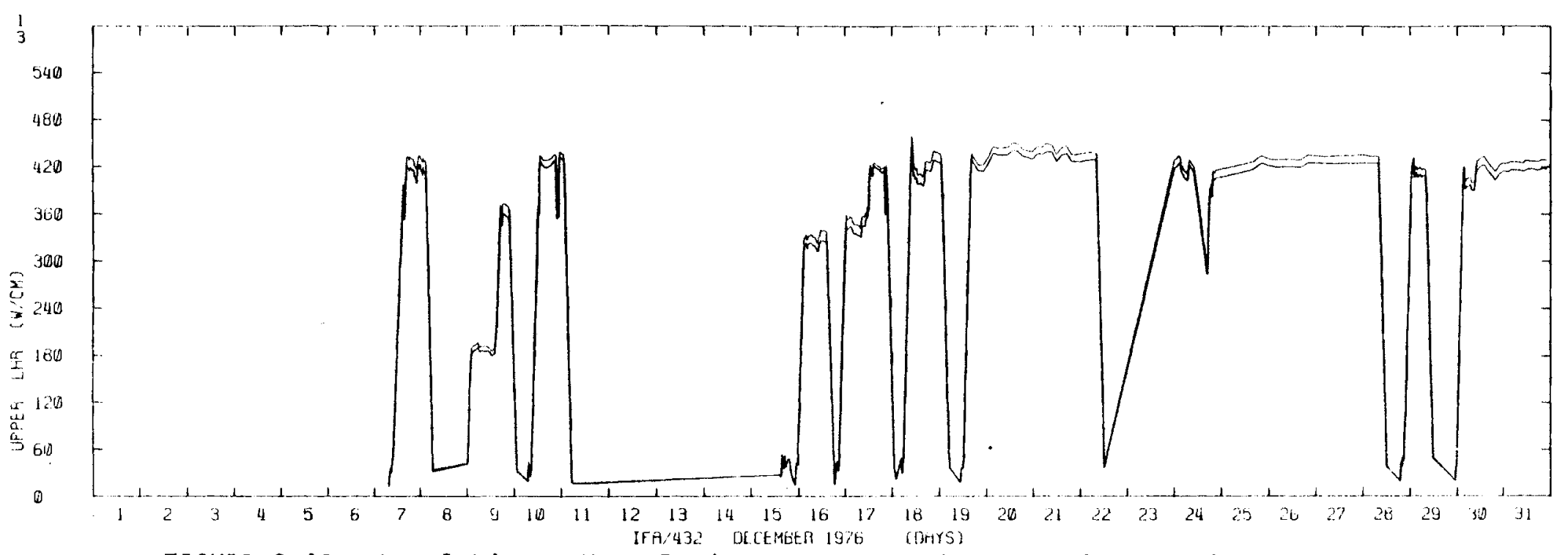

$w$
1
$N$

FIGURE 3.49. Local Linear Heat Ratings at Upper Thermocouple Locations for Rods 1,3 of IFA 432 - Decenber 1976

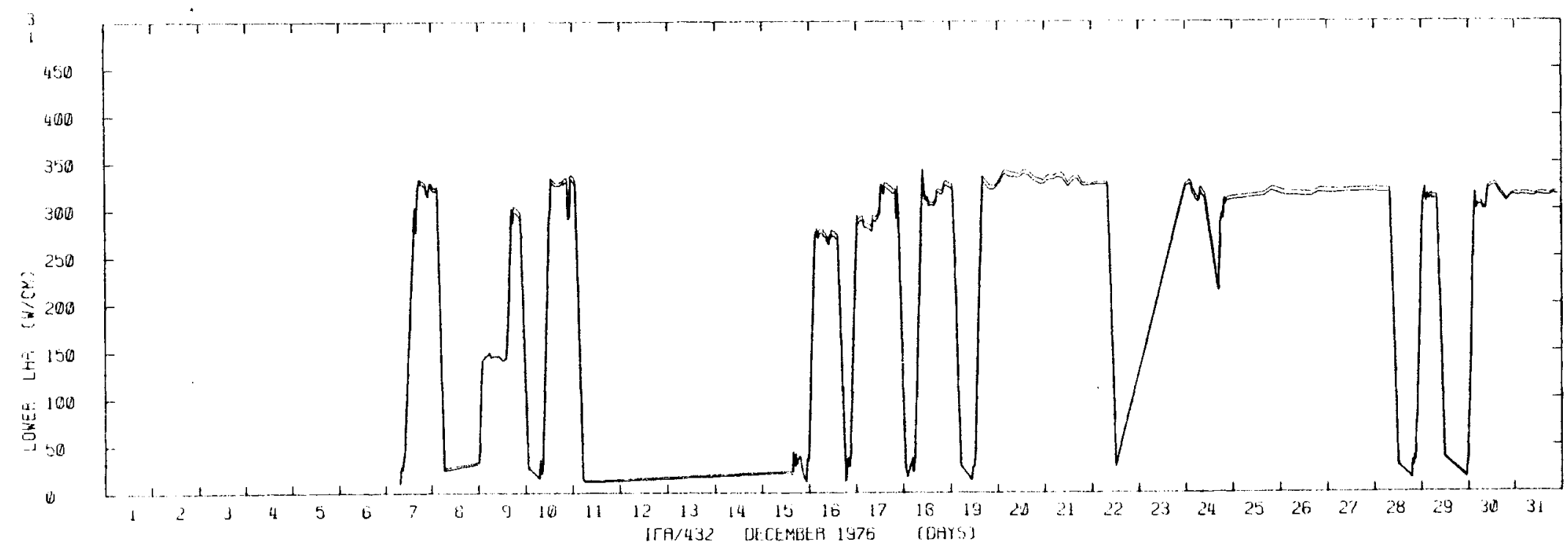

FIGURE 3.50. Local Linear Heat Ratings at Lower Thermocouple Locations for Rods 1,3 of IFA 432 - December 1976 


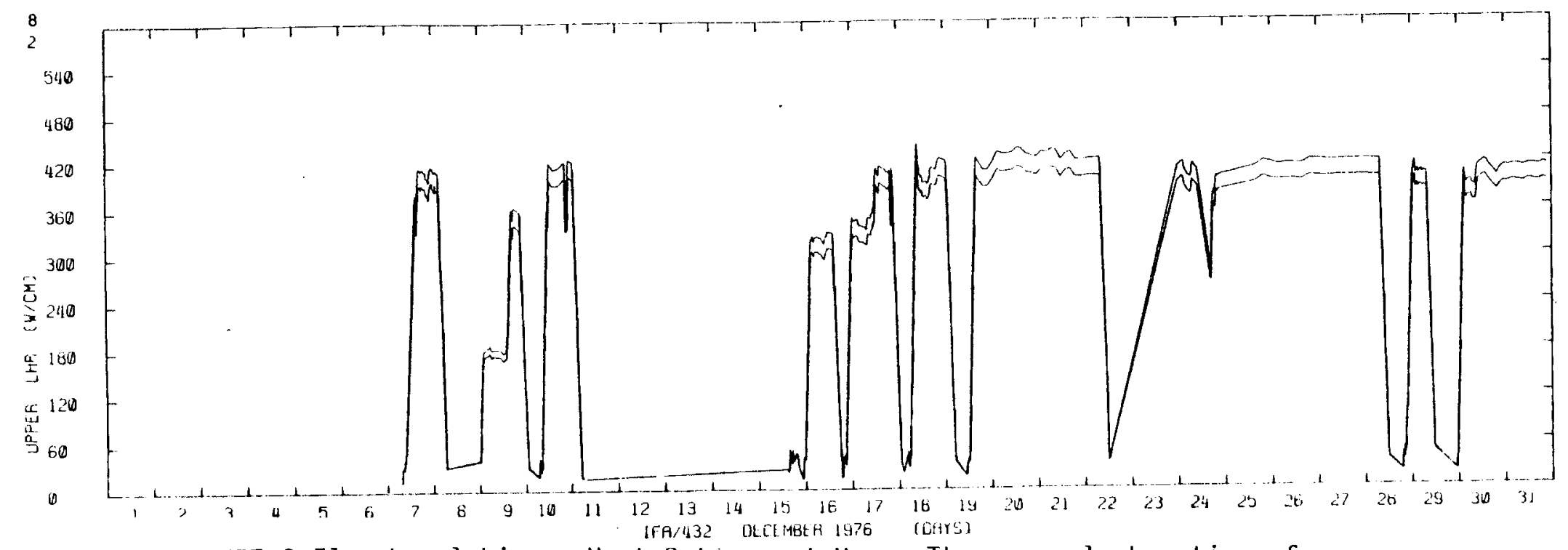

$\omega$
1
$\infty$

rIGURE 3.51. Local Linear Heat Ratings at Upper. Thermocouple Locations for Rods 2,8 of IFA 432 - December 1976

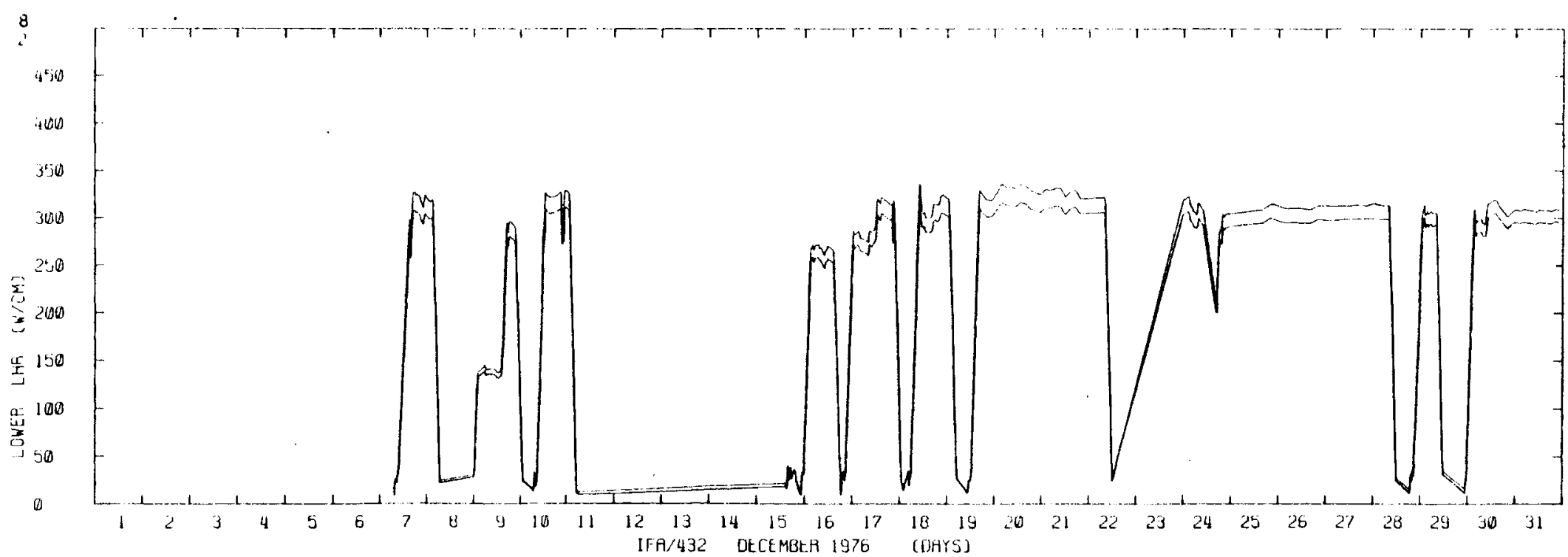

FIGURE 3.52. Local Linear Heat Ratings at Lower Thermocouplé Locations for Rods 2,8 of IFA 432 - December 1976 


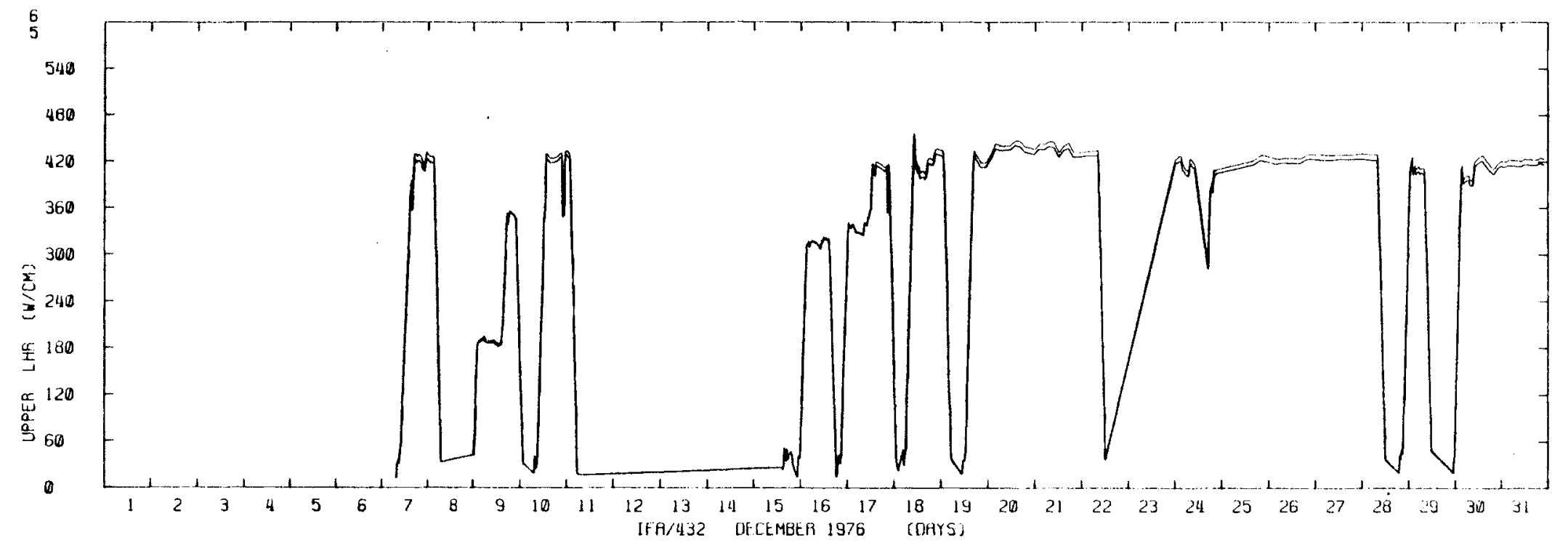

$\omega$
1
0

FIGURE 3.53. Local Linear Heat Ratings at Upper Thermocouple Locations for Rods 5,6 of IFA 432 - December 1976

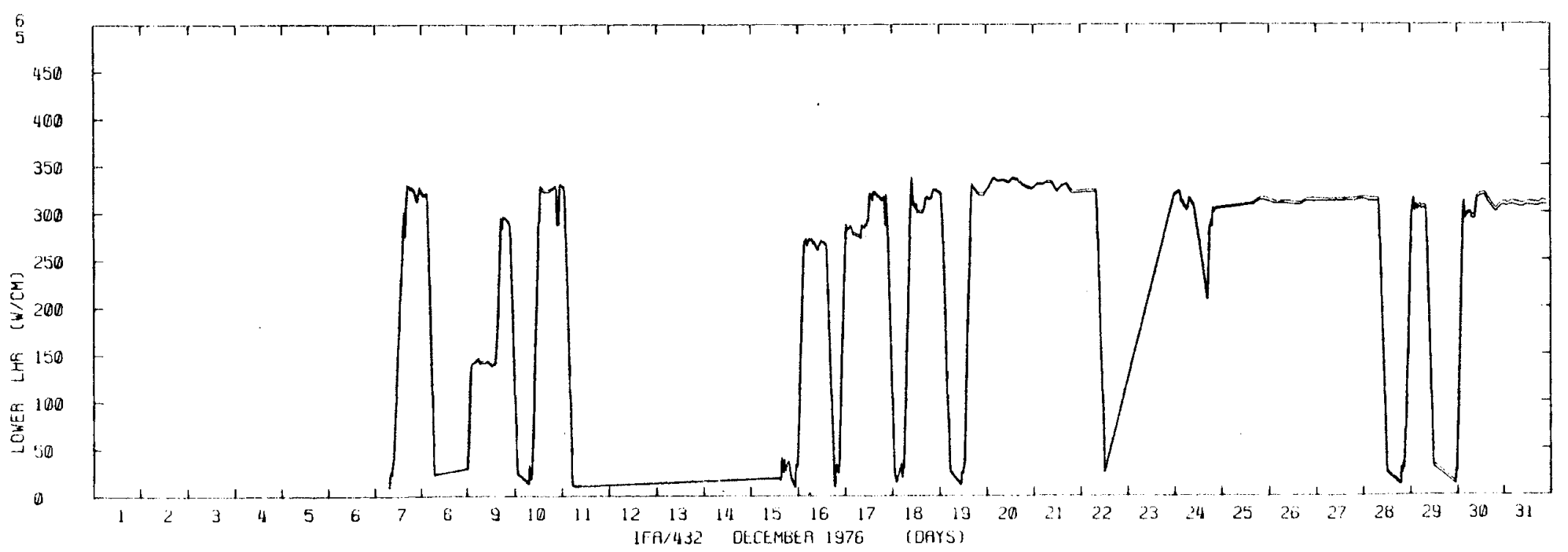

FIGURE 3.54. Local Linear Heat Ratings at Lower Thermocouple Locations for Rods 5,6 of IFA 432 - December 1976 


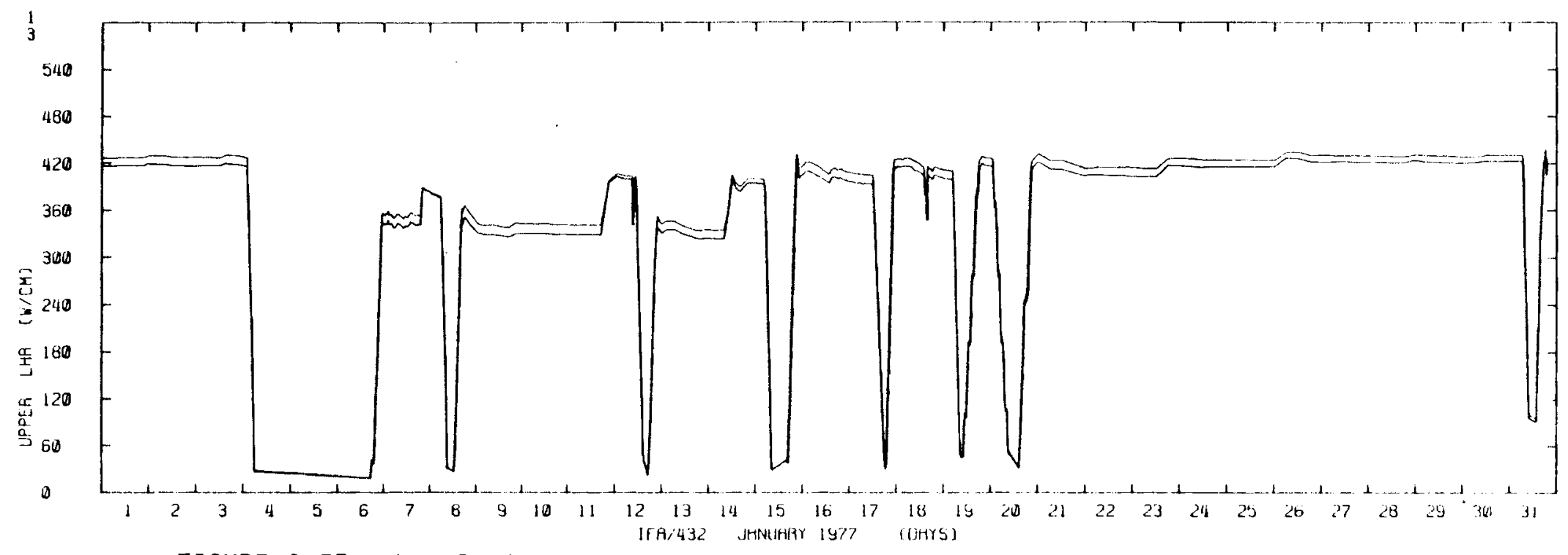

$\omega$
$\omega$
0

FIGURE 3.55. Local Linear Heat Ratings at Upper Thermocouple Locations for Rods 1,3 of IFA 432 - January 1977

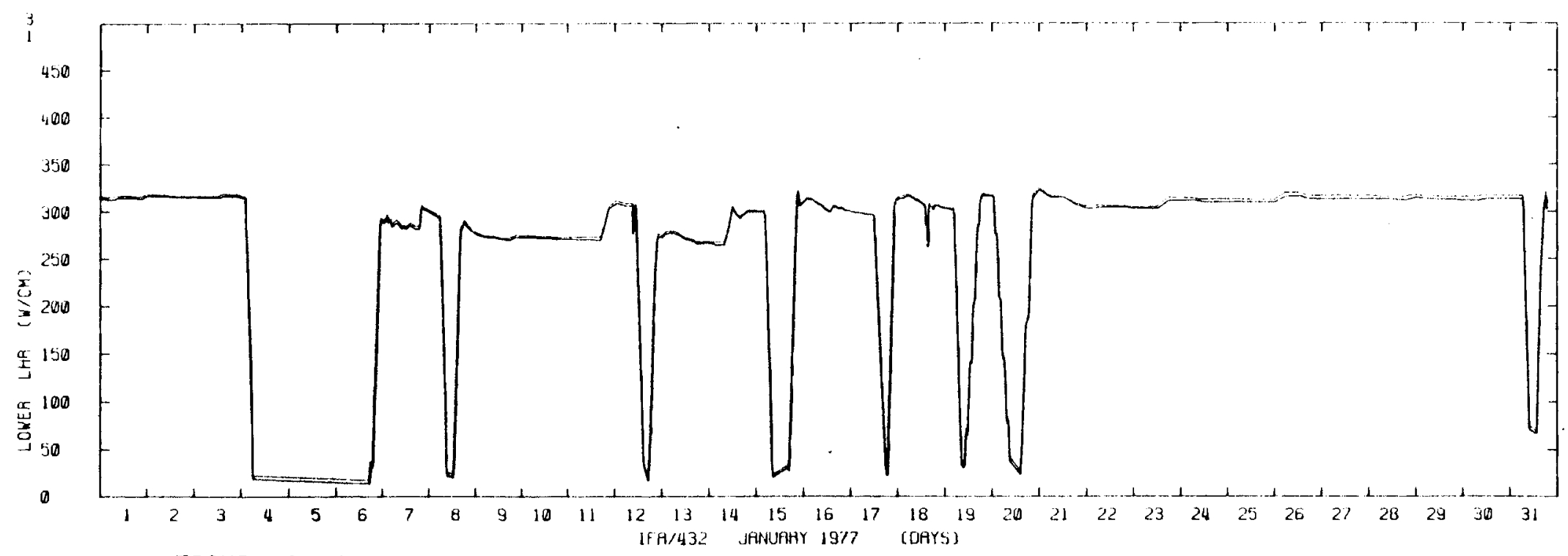

FIGURE 3.56. Local Linear Heat Ratings at Lower Thermocouple Locations for Rods 1,3 of IFA 432 - January 1977 


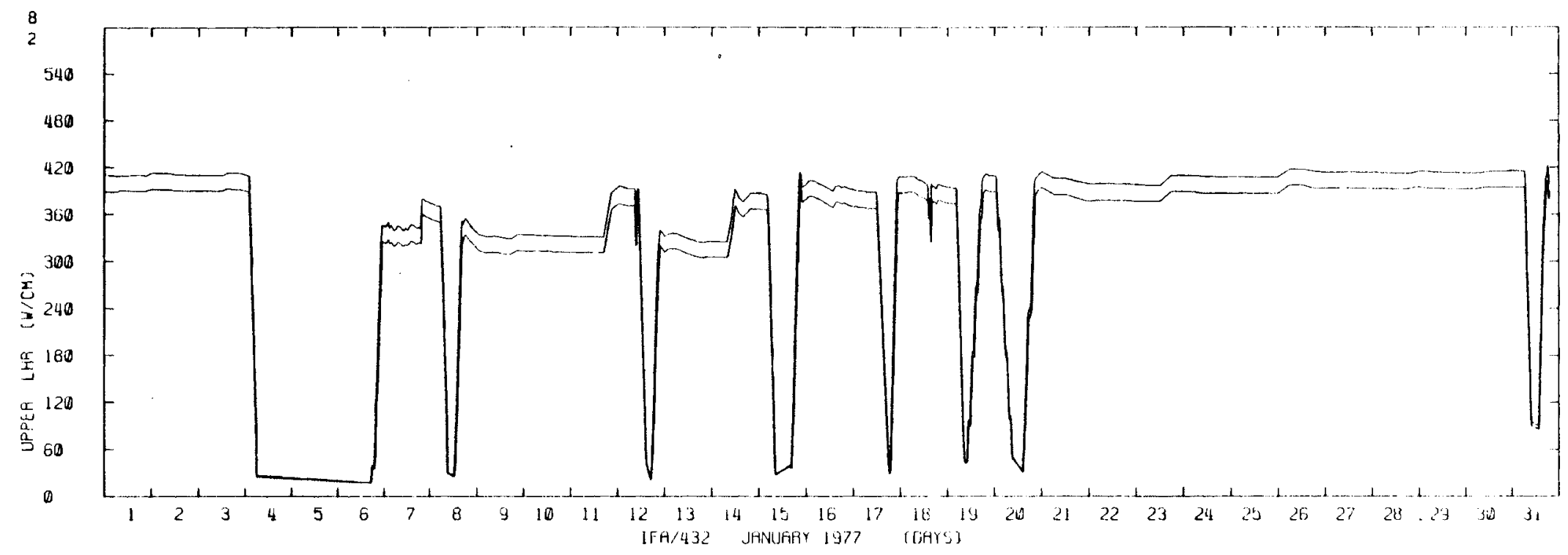

$\stackrel{\omega}{\omega}$

FIGURE 3.57. Local Linear Heat Ratings at Upper Thermocouple Locations for Rods 2,8 of IFA 432 - January 1977

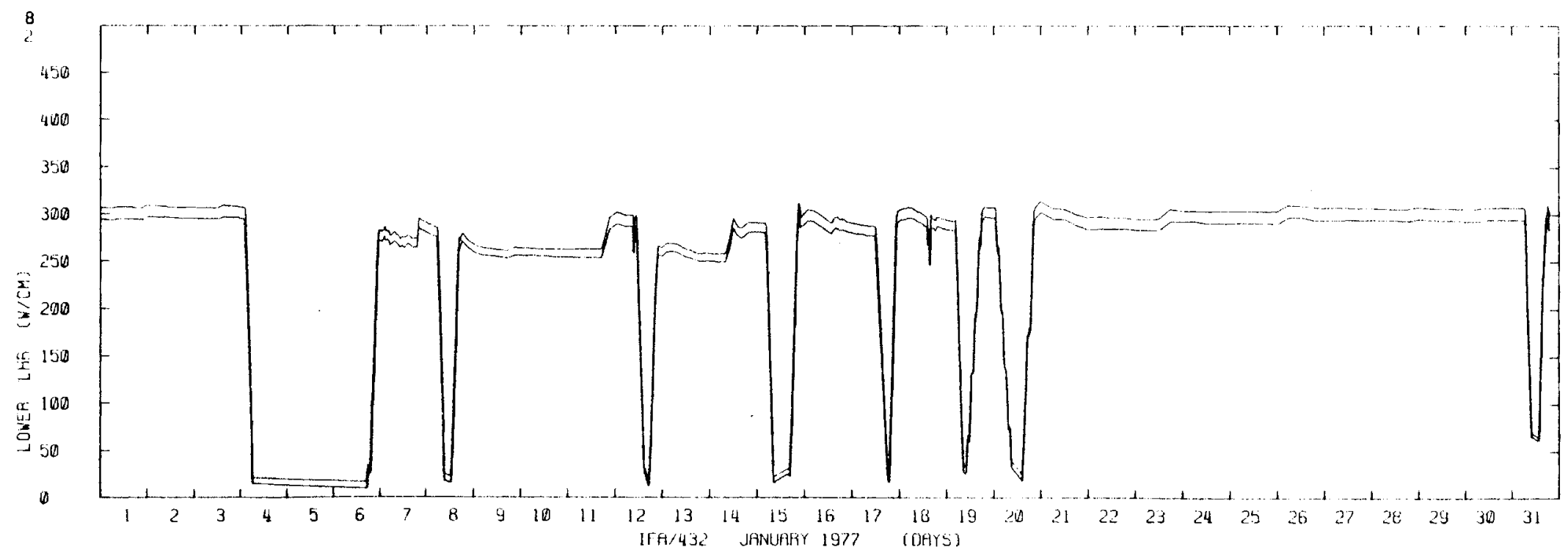

FIGURE 3.58. Local Linear Heat Ratings at Lower Thermocouple Locations for Rods 2,8 of IFA 432 - January 1977 


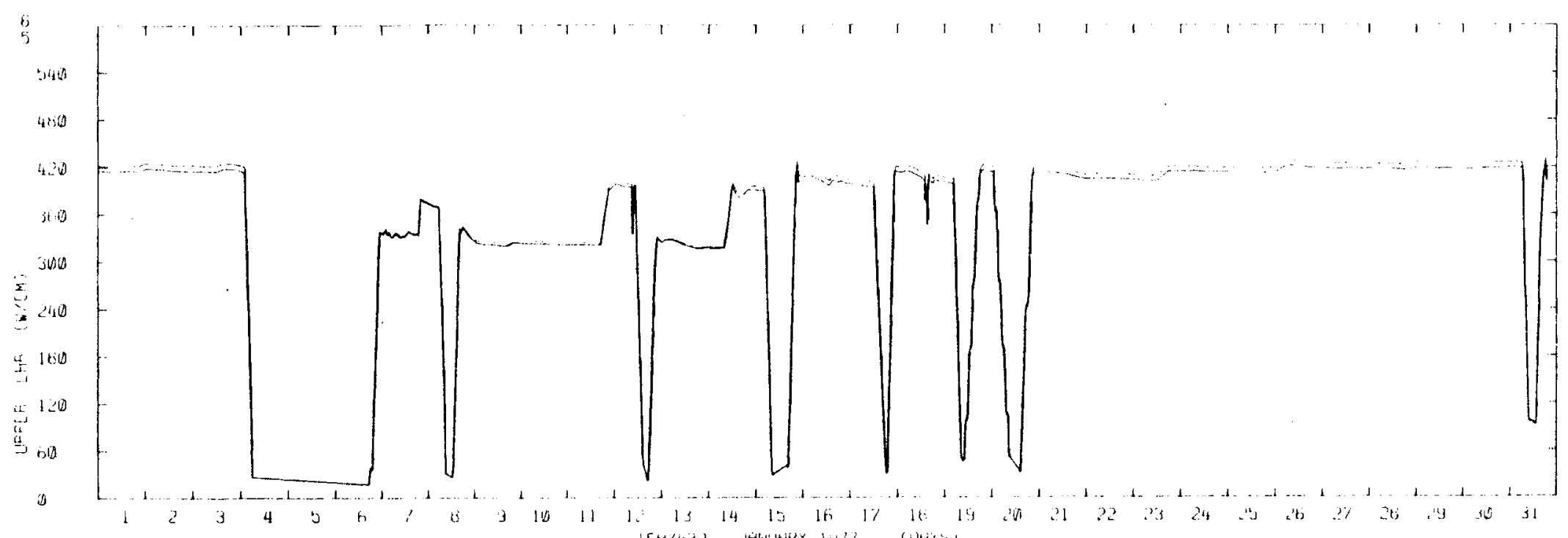

FIGURE 3.59. Local Linear Heat Ratings at Upper Thermocouple Locations for Rods 5,6 of IFA 432 - January 1977

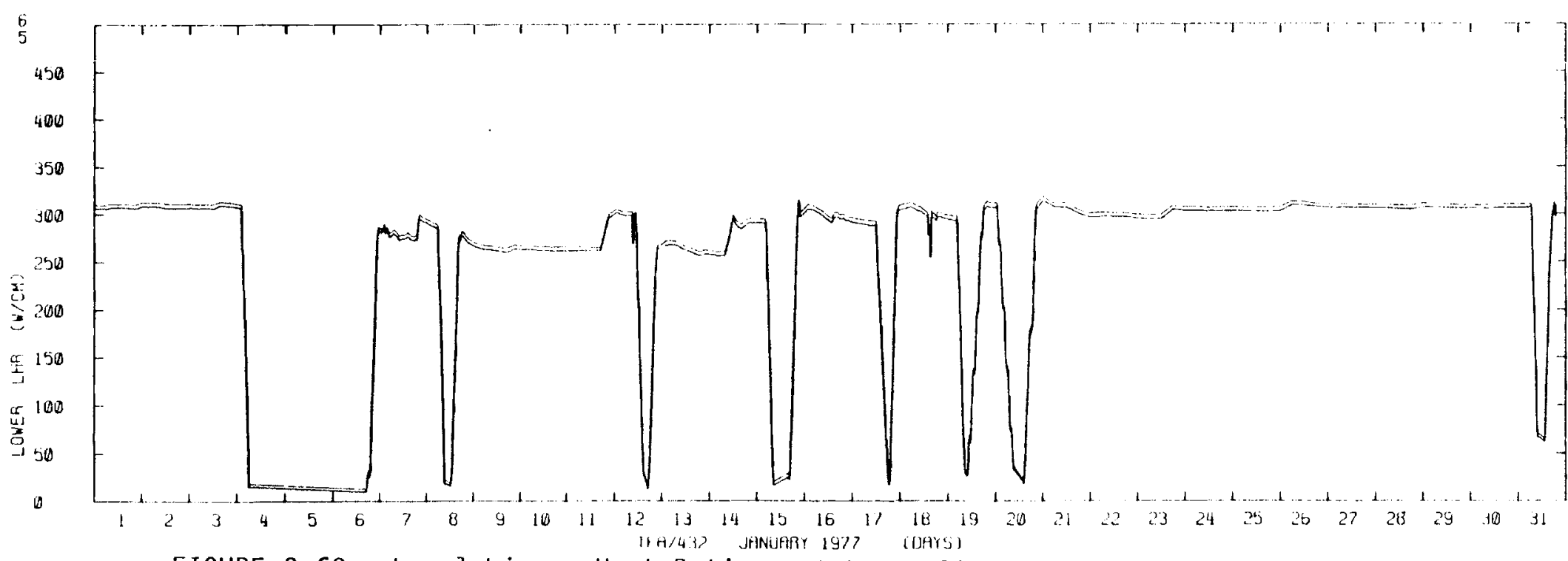

FIGURE 3.60. Local Linear Heat Ratings at Lower Thermocouple Locations for Rods 5,6 of IFA 432 - January 1977 


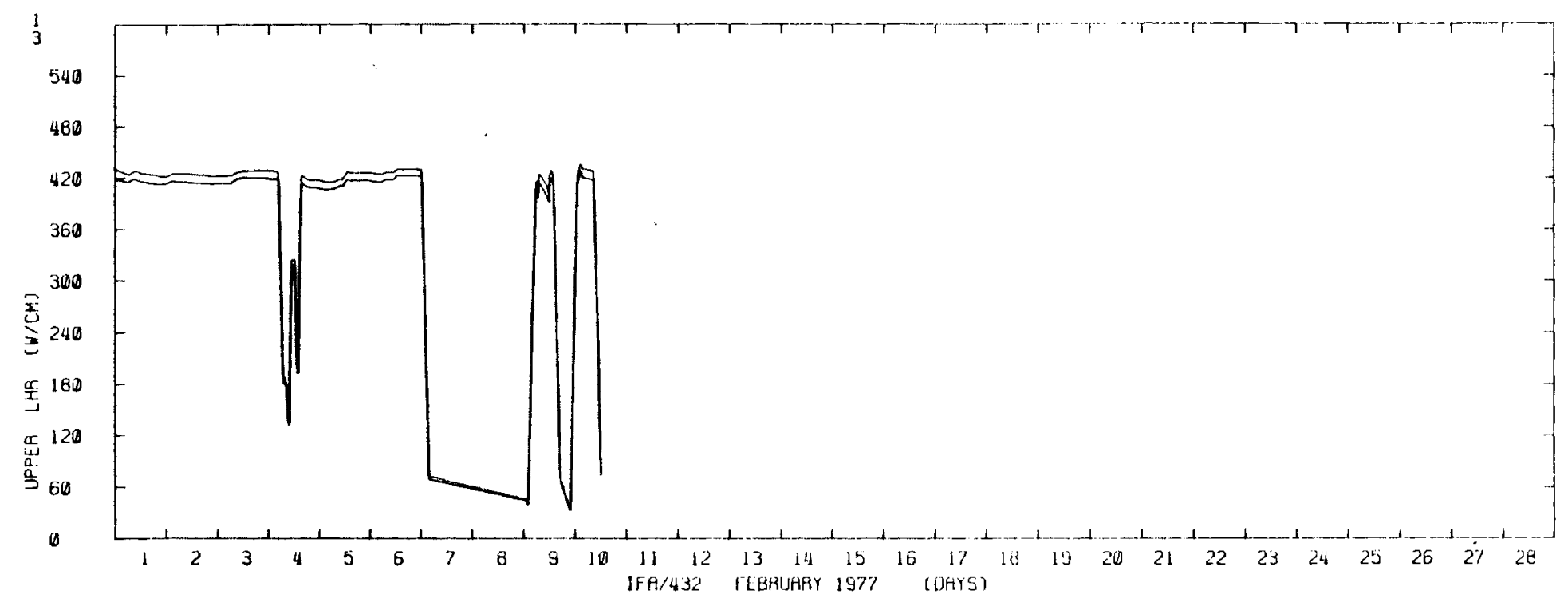

FIGURE 3.61. Local Linear Heat Ratings at Upper Thermocouple Locations for Rods 1,3 of IFA 432 - February 1977

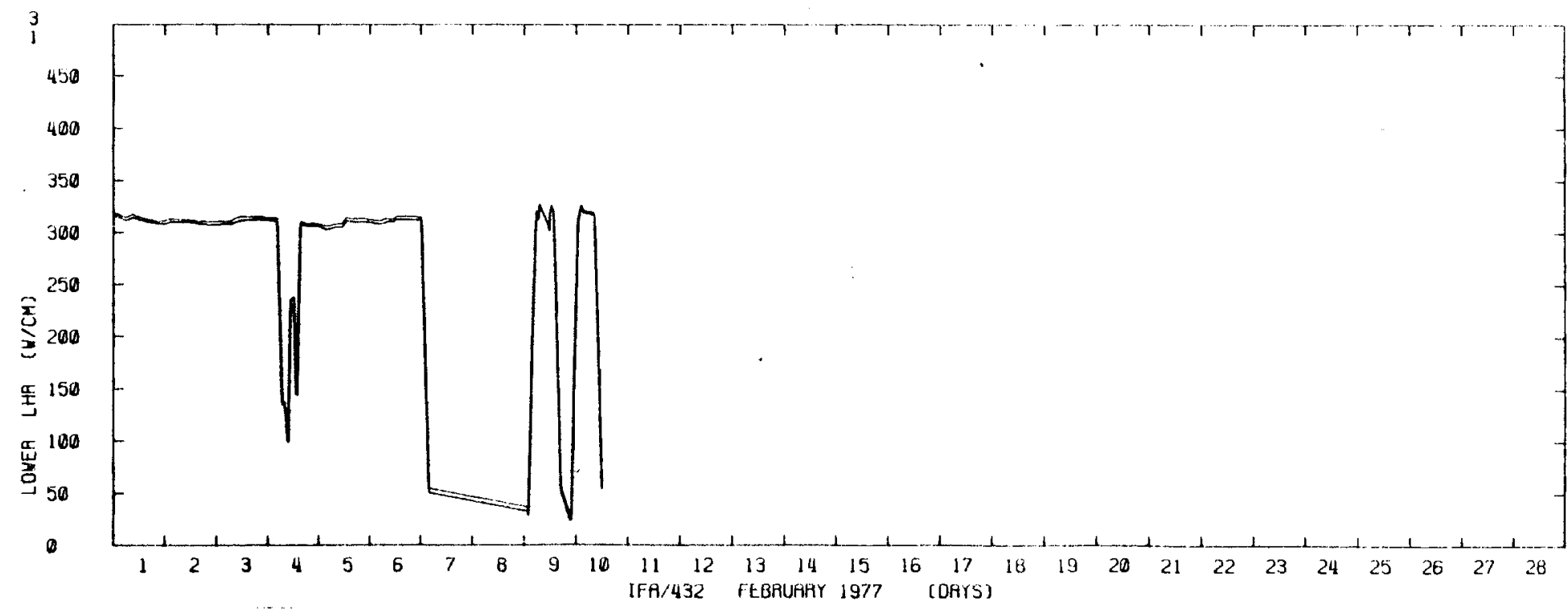

FIGURE 3.62. Local Linear Heat Ratings at Lower Thermocouple Locations for Rods 1,3 of IFA 432 - February 1977 


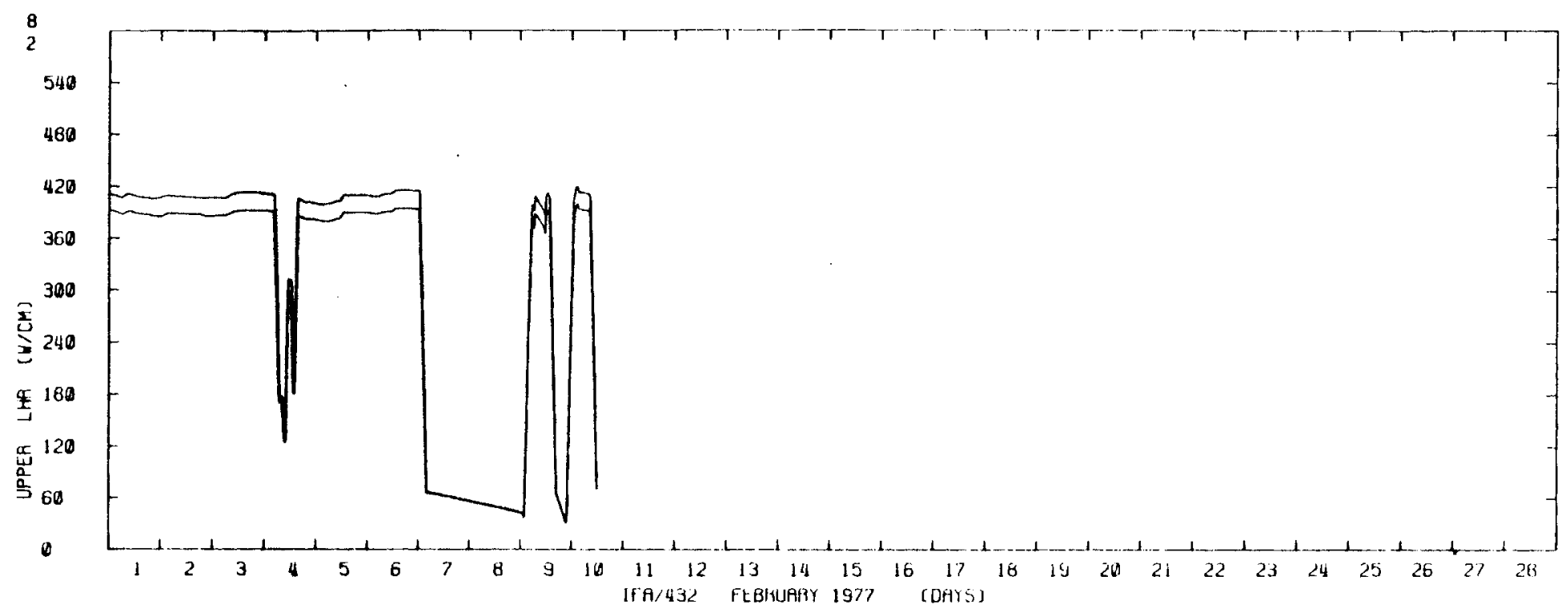

FIGURE 3.63. Local Linear Heat Ratings at Upper Thermocouple Locations for Rods 2,8 of IFA 432 - February 1977

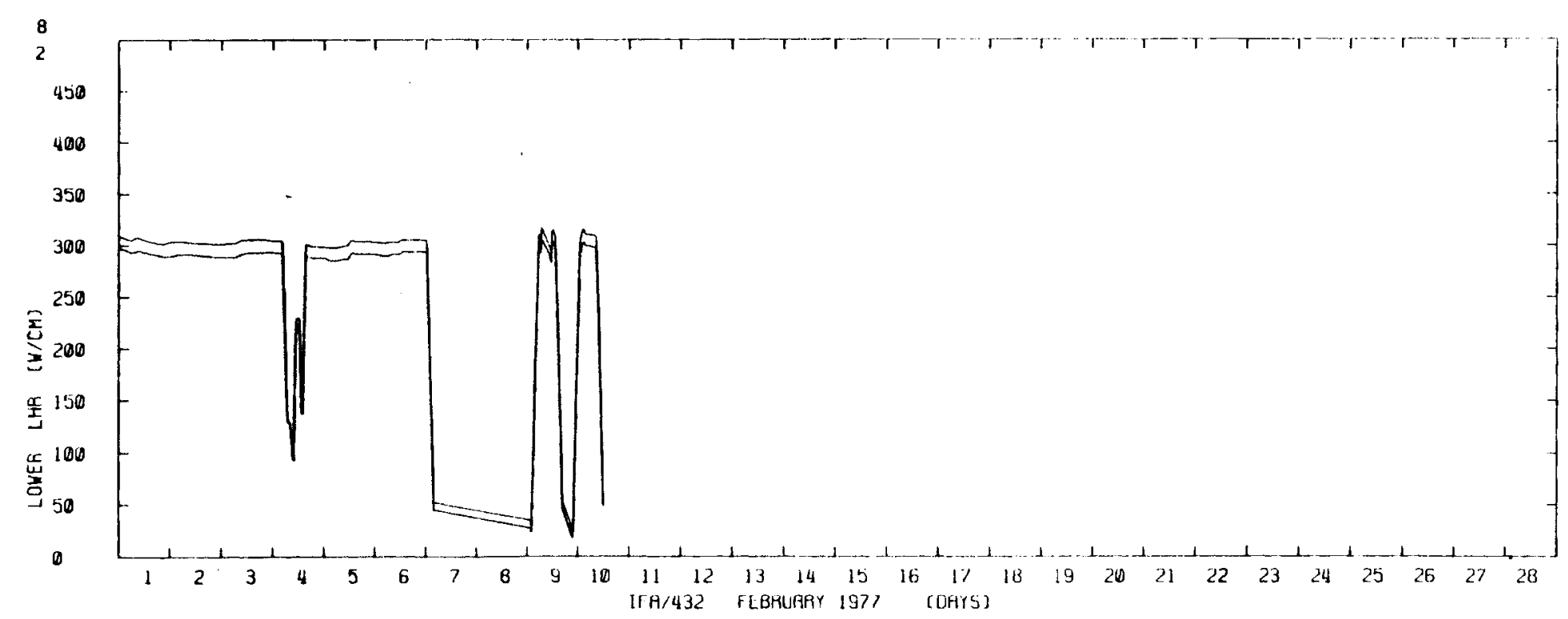

FIGURE 3.64. Local Linear Heat Ratings at Lower Thermocouple Locations for Rods 2,8 of IFA 432 - February 1977 


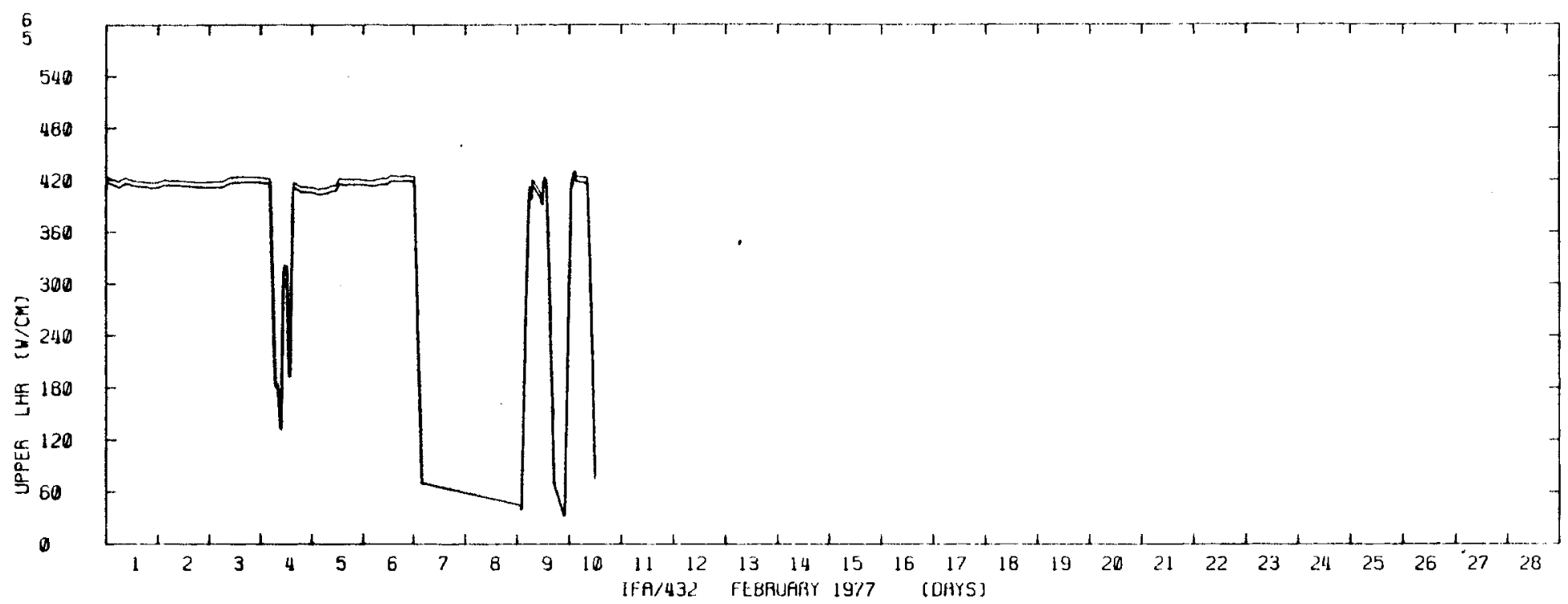

FIGURE 3.65. Local Linear Heat Ratings at Upper Thermocouple Locations for Rods 5,6 of IFA 432 - February 1977

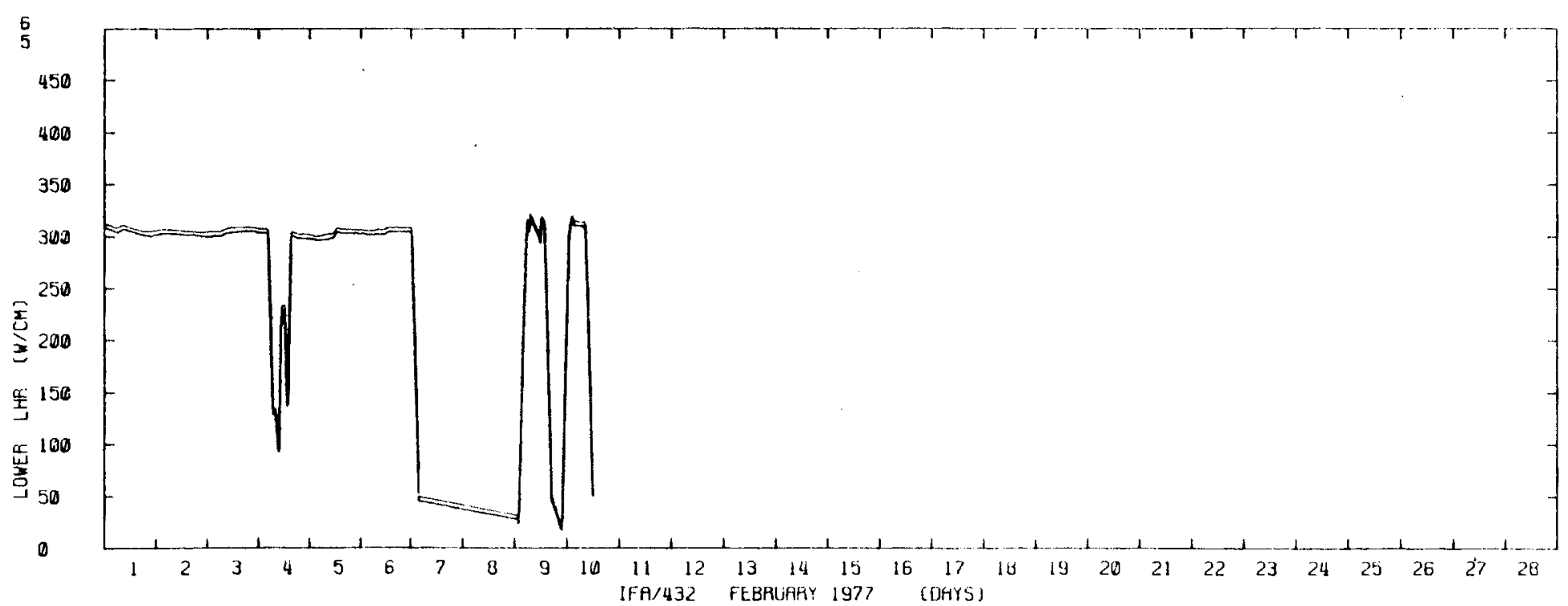

FIGURE 3.66. Local Linear Heat Ratings at Lower Thermocouple Locations for Rods 5,6 of IFA 432 - February 1977 


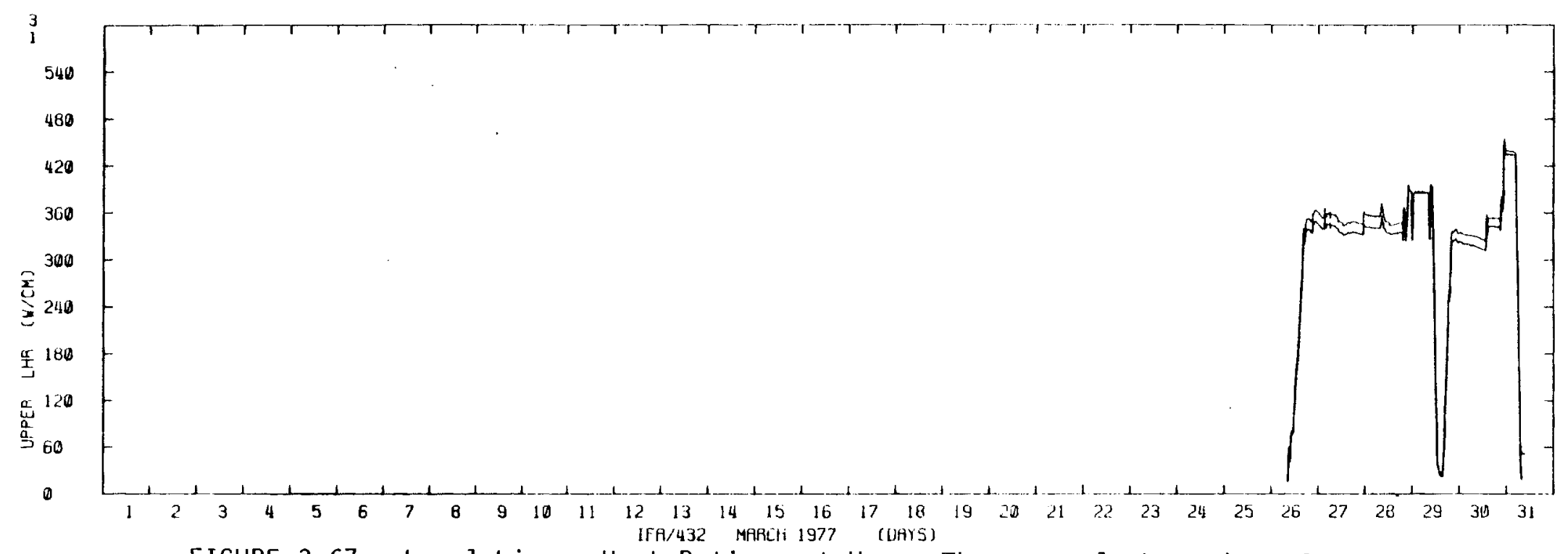

$\stackrel{\omega}{\omega}$

FIGURE 3.67. Local Linear Heat Ratings at Upper Thermocouple Locations for Rods 1,3 of IFA 432 - March 1977

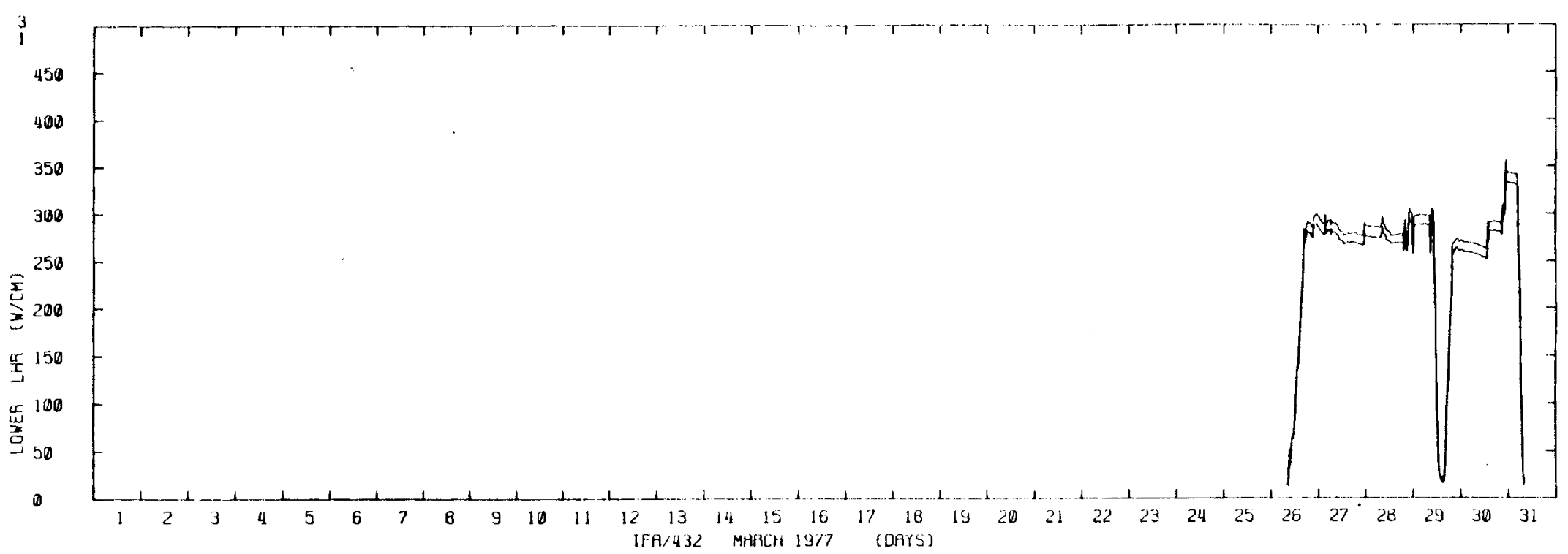

FIGURE 3.68. Local Linear Heat Ratings at Lower Thermocouple Locations for Rods 1,3 of IFA 432 - March 1977 


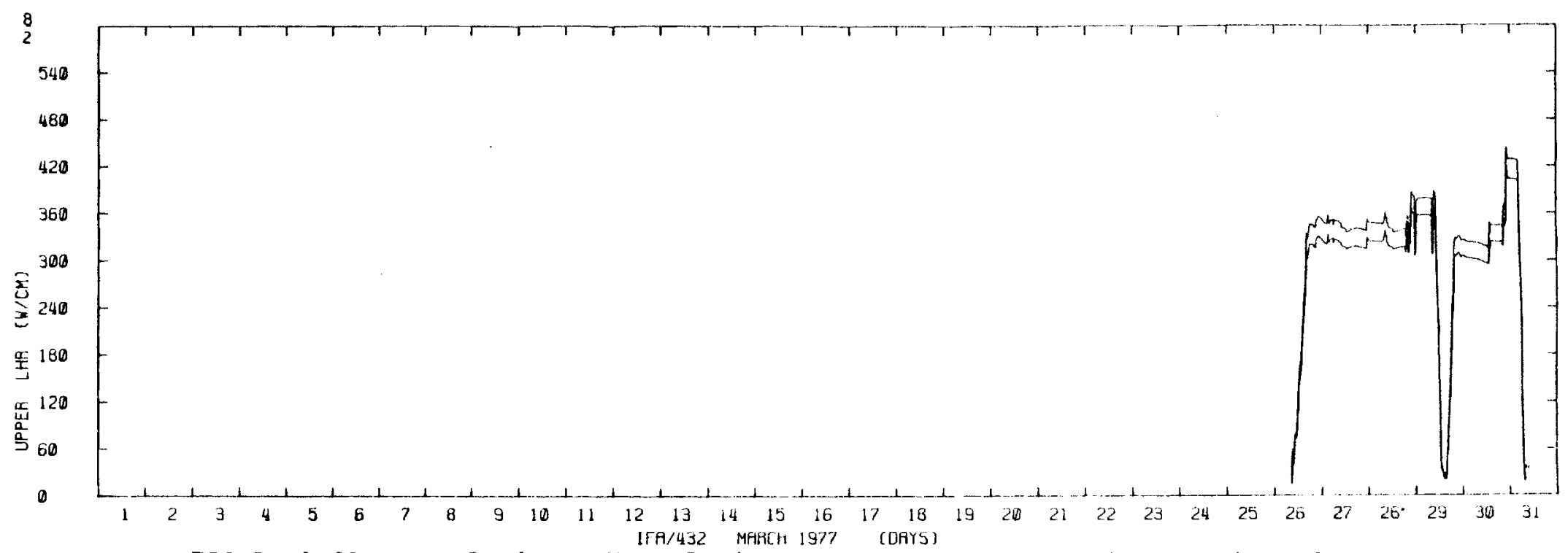

$\omega$
$\omega$
$w$

FIGURE 3.69. Local Linear Heat Ratings at Upper Thermocouple Locations for Rods 2,8 of IFA 432 - March 1977

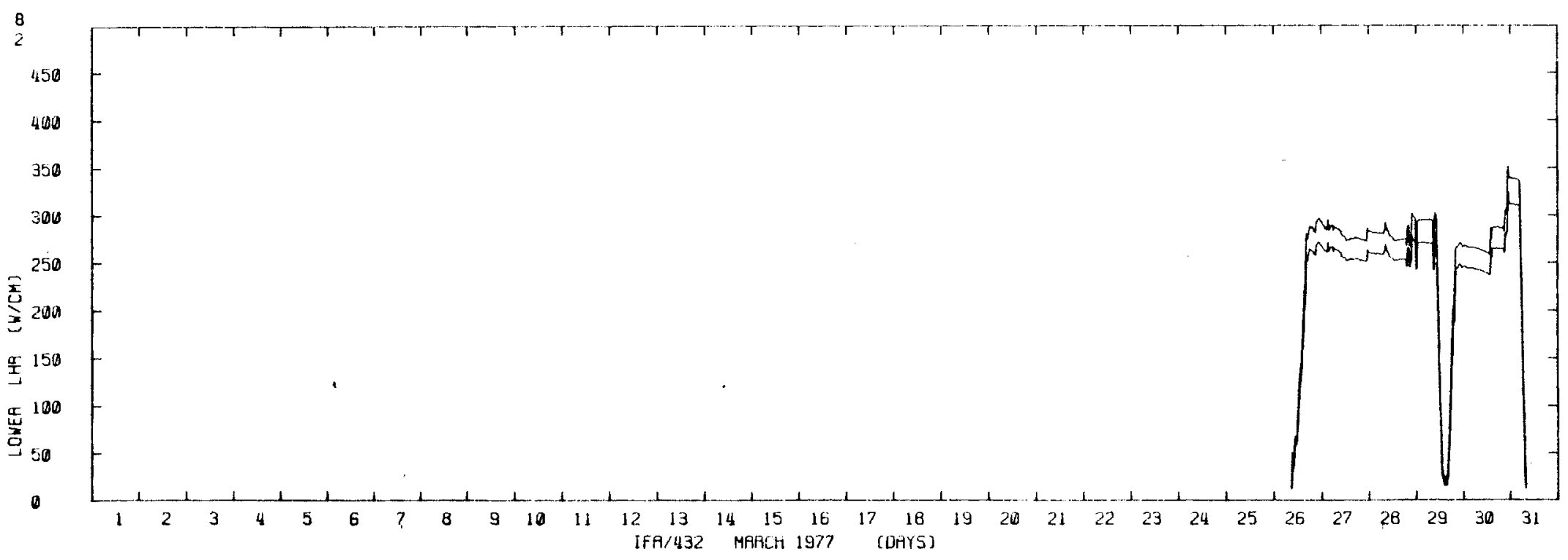

FIGURE 3.70. Local Linear Heat Ratings at Lower Thermocouple Locations for Rods 2,8 of IFA 432 - March 1977 


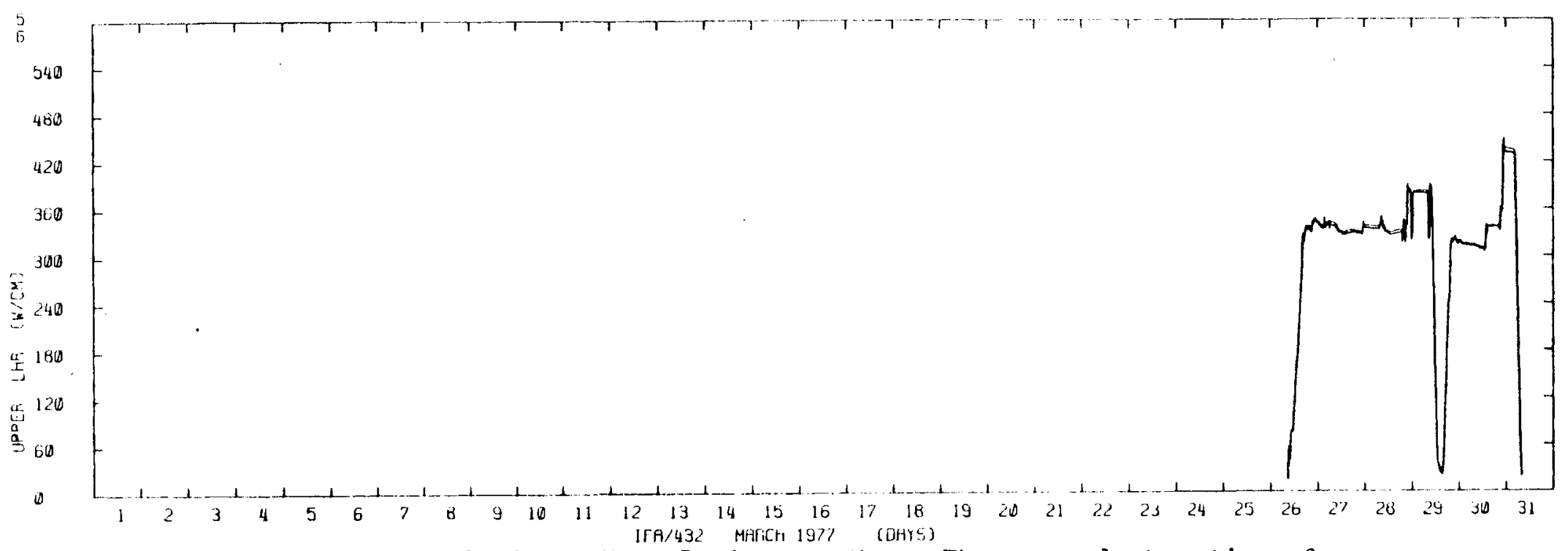

FIGURE 3.71. Local Linear Heat Ratings at Upper Thermocouple Locations for Rods 5,6 of IFA 432 - March 1977

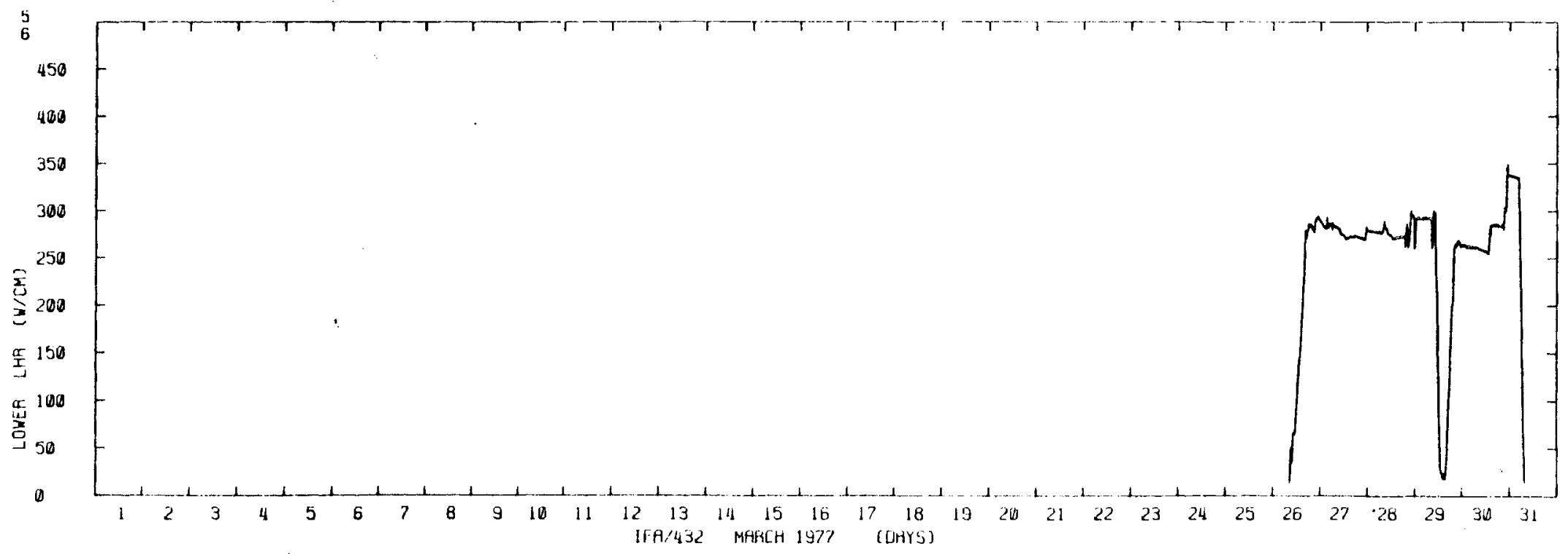

FIGURE 3.72. Local Linear Heat Ratings at Lower Thermocouple Locations for Rods 5,6 of IFA 432 - March 1977 


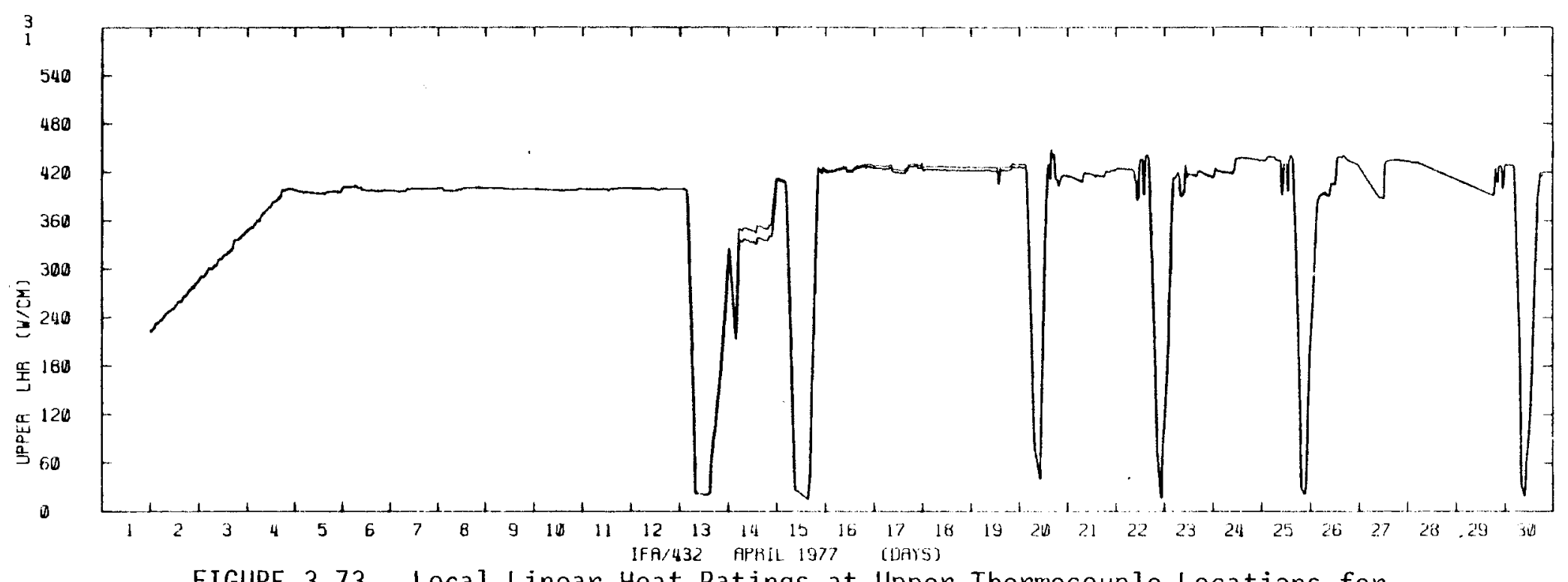

$\omega$

FIGURE 3.73. Local Linear Heat Ratings at Upper Thermocouple Locations for Rods 1,3 of IFA 432 - Apri1 1977

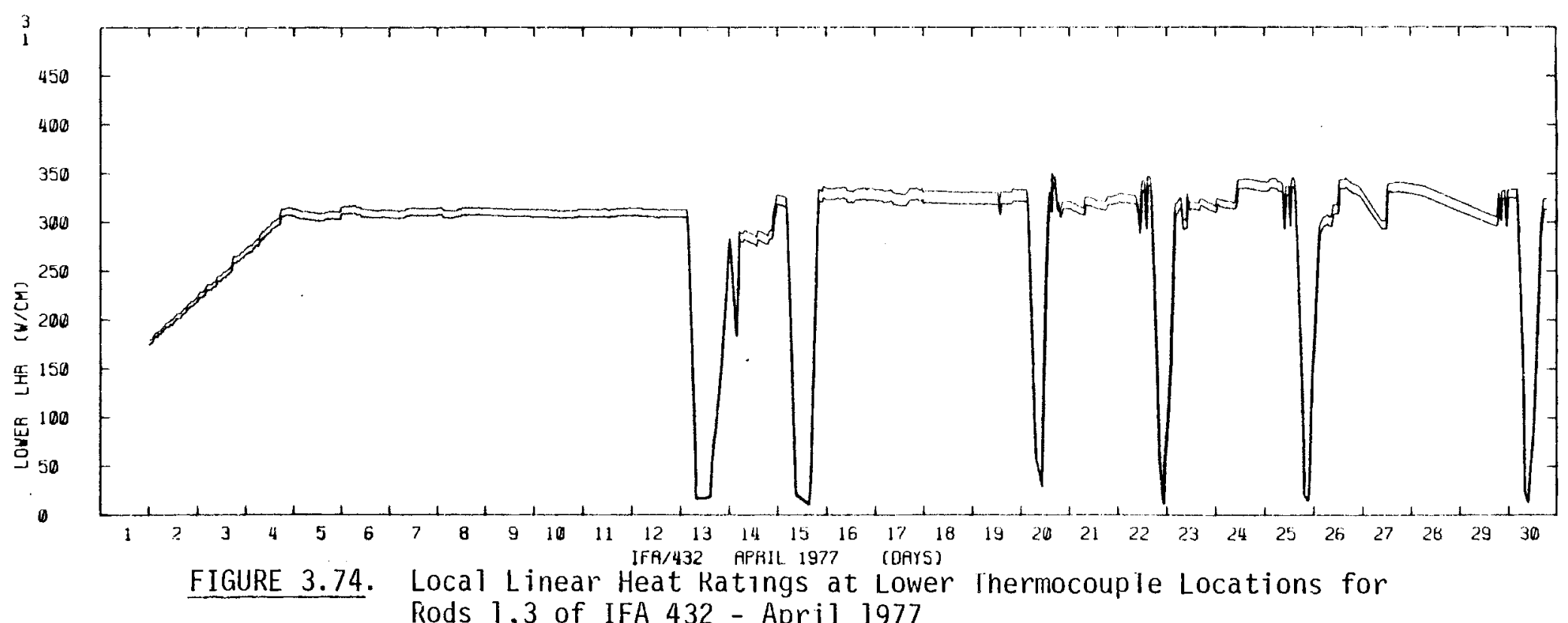




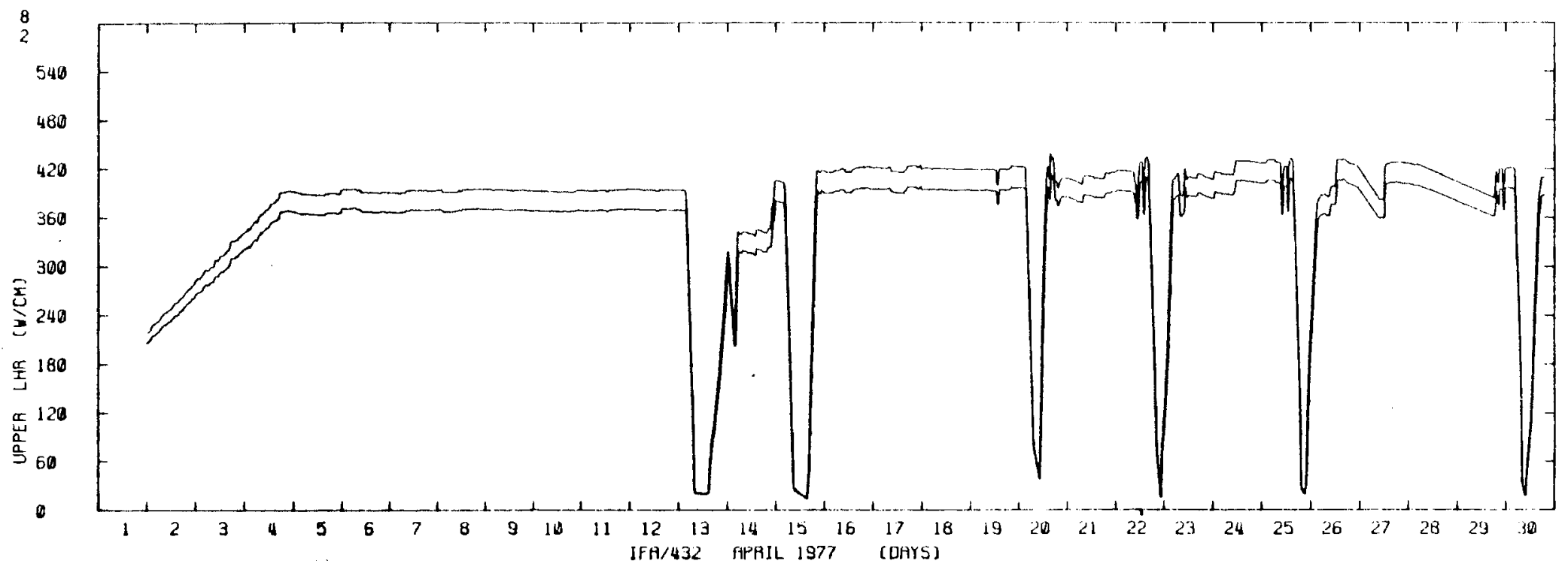

$\omega$
1
0

FIGURE 3.75. Local Linear Heat Ratings at Upper Thermocouple Locations for Rods 2,8 of IFA 432 - April 1977

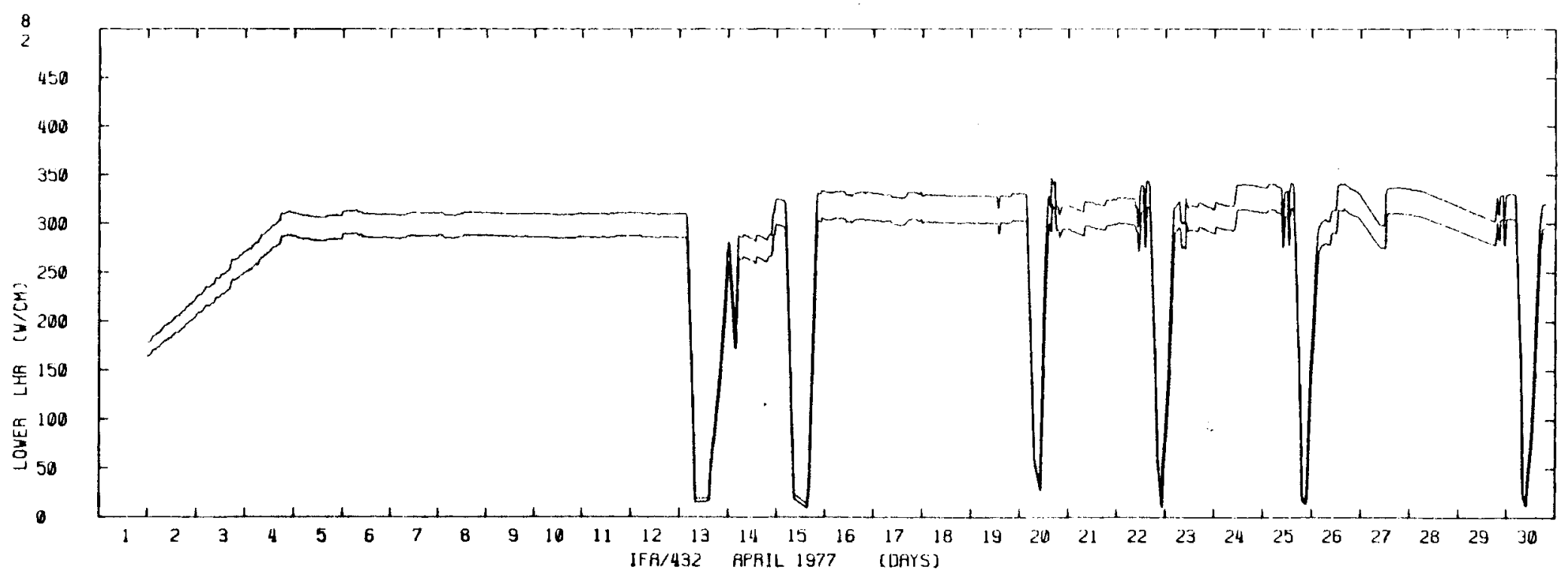

FIGURE 3.76. Local Linear Heat Ratings at Lower Thermocouple Locations for Rods 2,8 of IFA 432 - Apri1 1977 


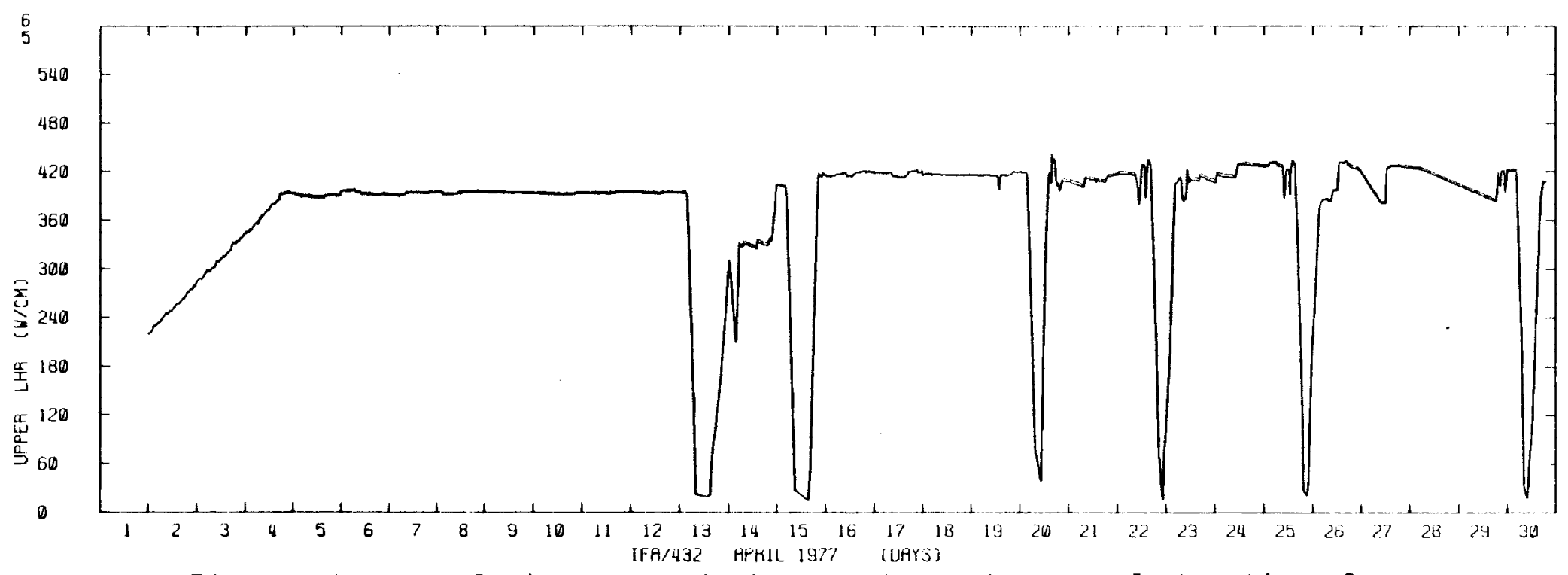

$\omega$
1

FIGURE 3.77. Local Linear Heat Ratings at Upper Thermocouple Locations for Rods 5,6 of IFA 432 - Apri1 1977

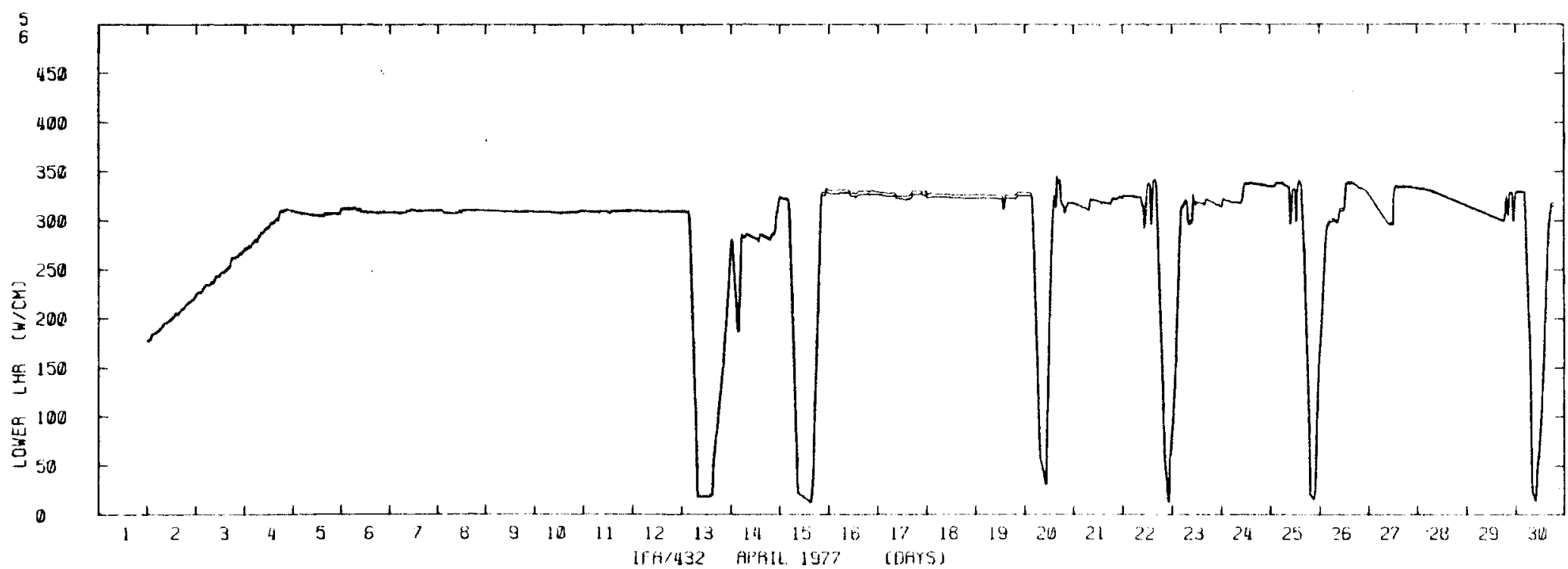

FIGURE 3.78. Local Linear Heat Ratings at Lower Thermocouple Locations for Rods 5,6 of IFA 432 - Apri 11977 


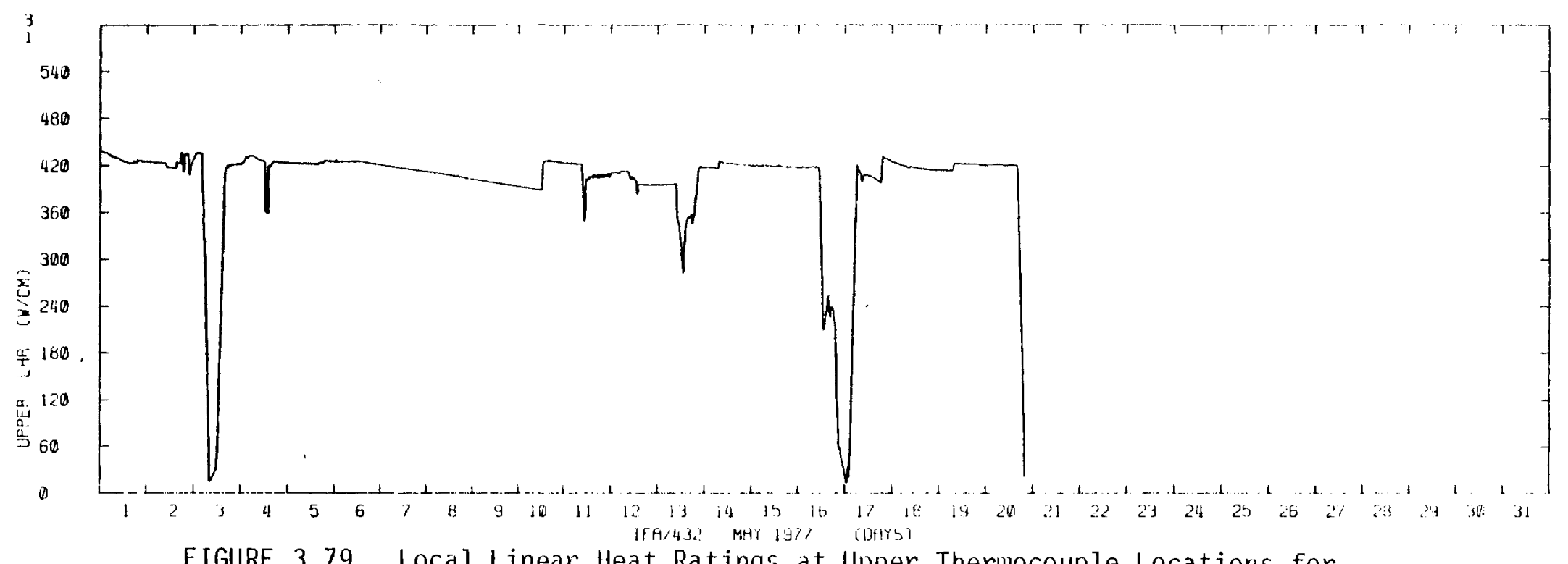

$\omega$
$\dot{1}$
$\stackrel{N}{N}$

FIGURE 3.79. Local Linear Heat Ratings at Upper Thermocouple Locations for Rods 1,3 of IFA 432 - May 1977

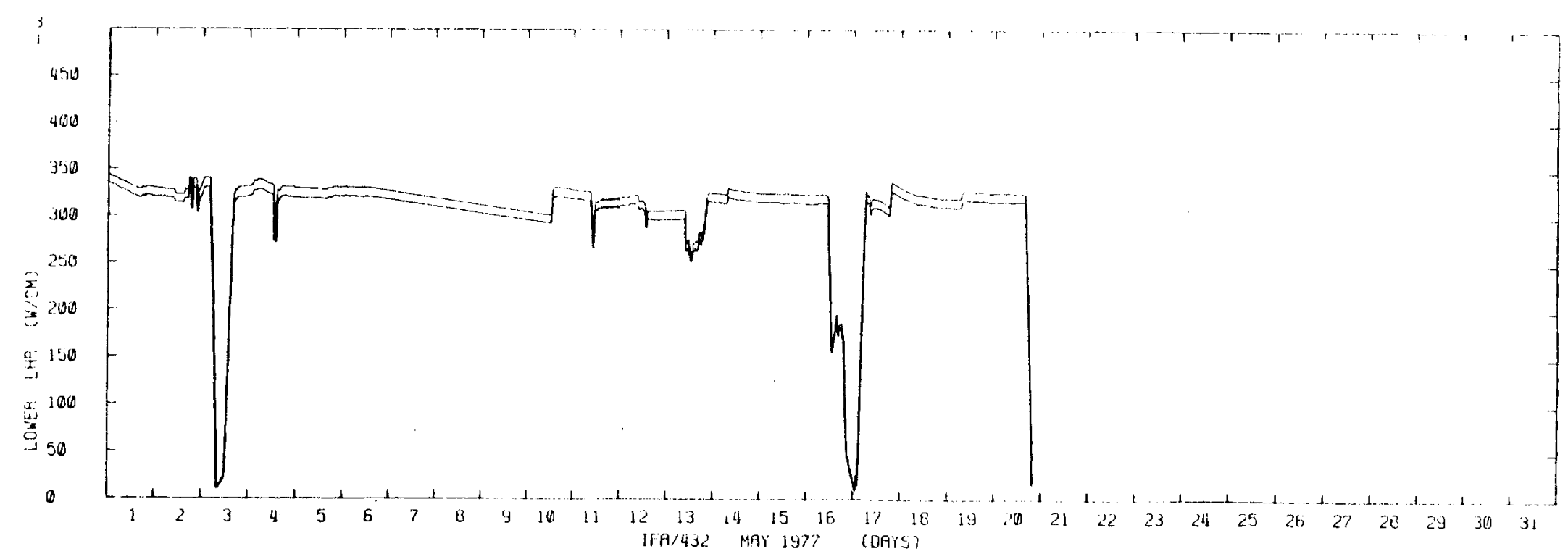

FIGURE 3.80. Local Linear Heat Ratings at Lower Thermocouple Locations for Rods 1,3 of IFA 432 - May 1977 


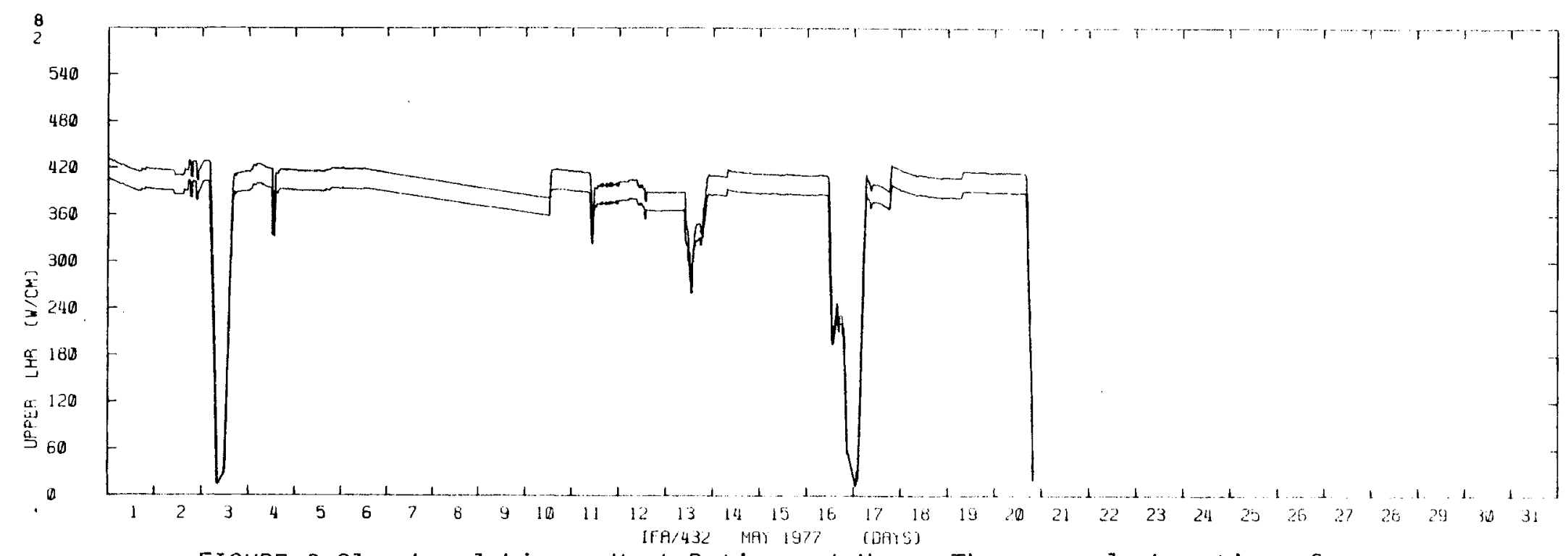

$\omega$
1
$\omega$

FIGURE 3.81. Local Linear Heat Ratings at Upper Thermocouple Locations for Rods 2,8 of IFA 432 - May 1977

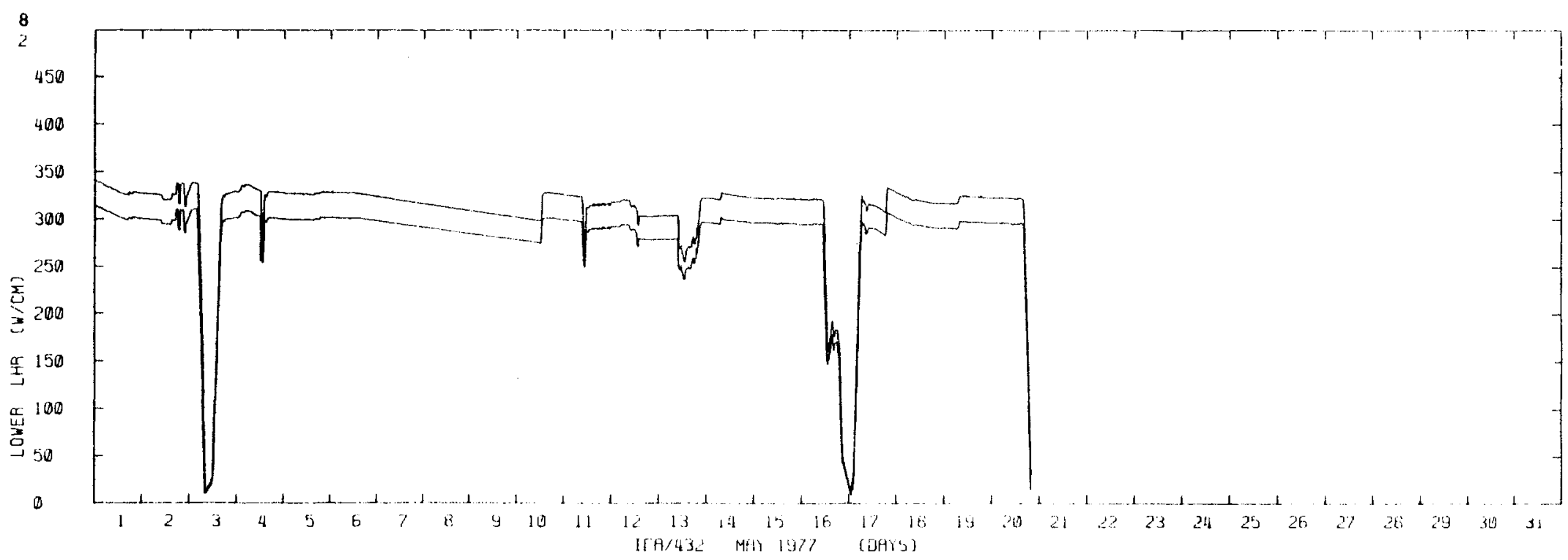

FIGURE 3.82. Local Linear Heat Ratings at Lower Thermocouple Locations for Rods 2,8 of IFA 432 - Hay 1977 


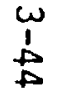

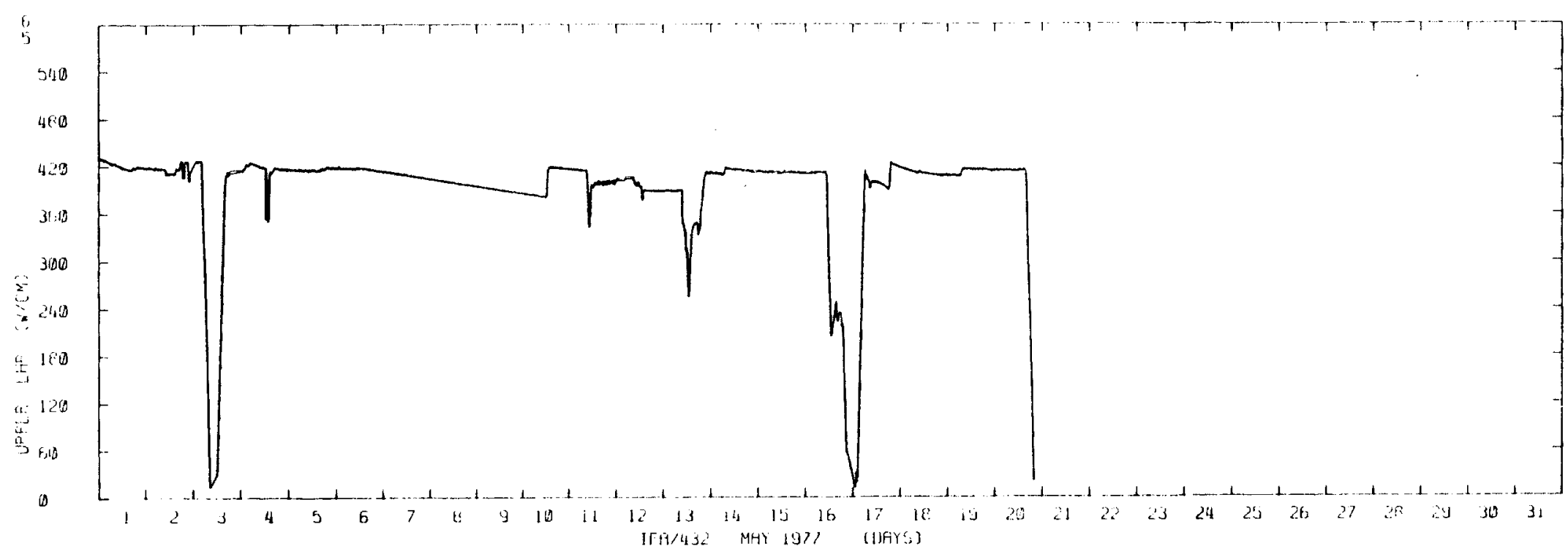

FIGURE 3.83. Local Linear Heat Ratings at Upper Thernocouple Locations for Rods 5,6 of IFA 432 - May 1977

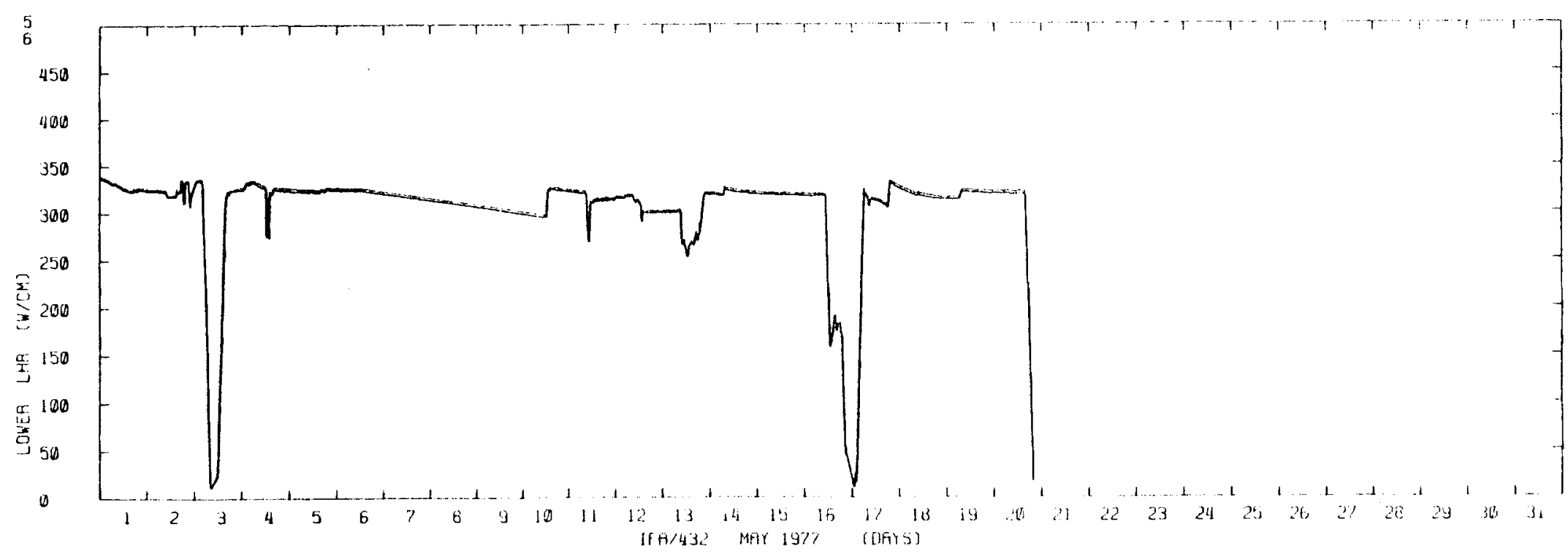

FIGURE 3.84. Local Linear Heat Ratings at Lower Thermocouple Locations for Rods 5,6 of IFA 432 - May 1977 


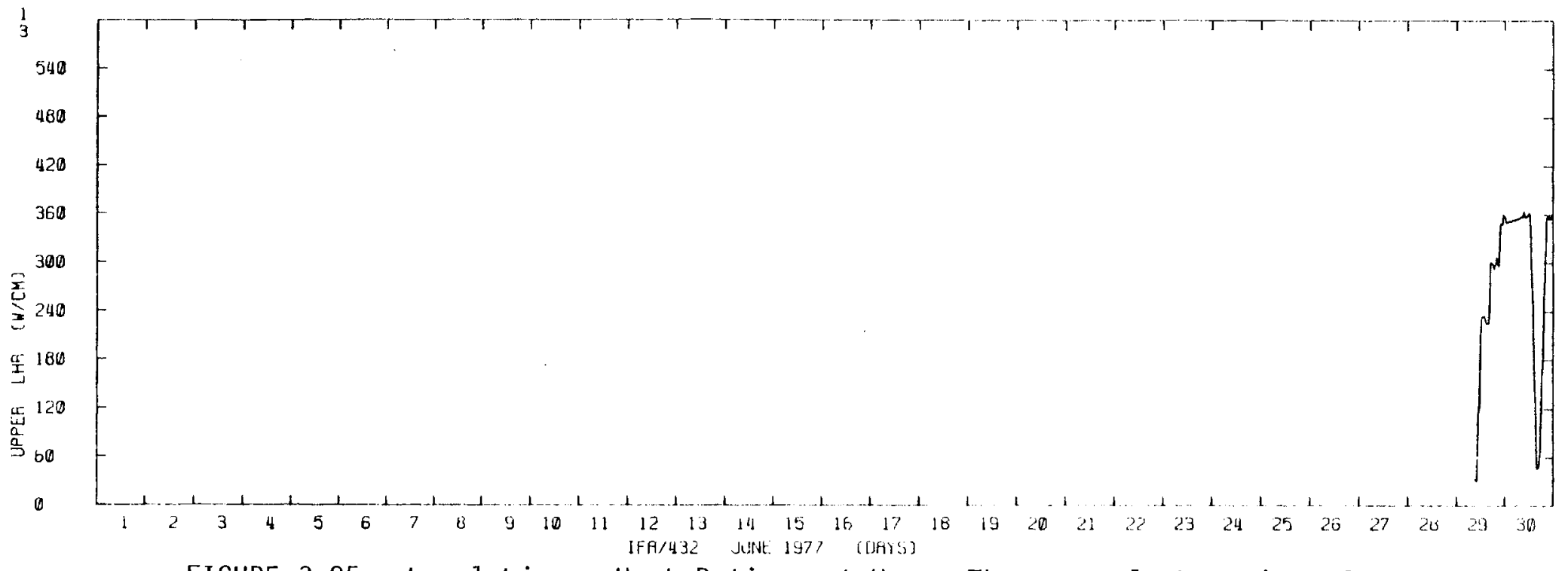

FIGURE 3.85. Local Linear Heat Ratings at Upper Thermocouple Locations for Rods 1,3 of IFA 432 - June 1977

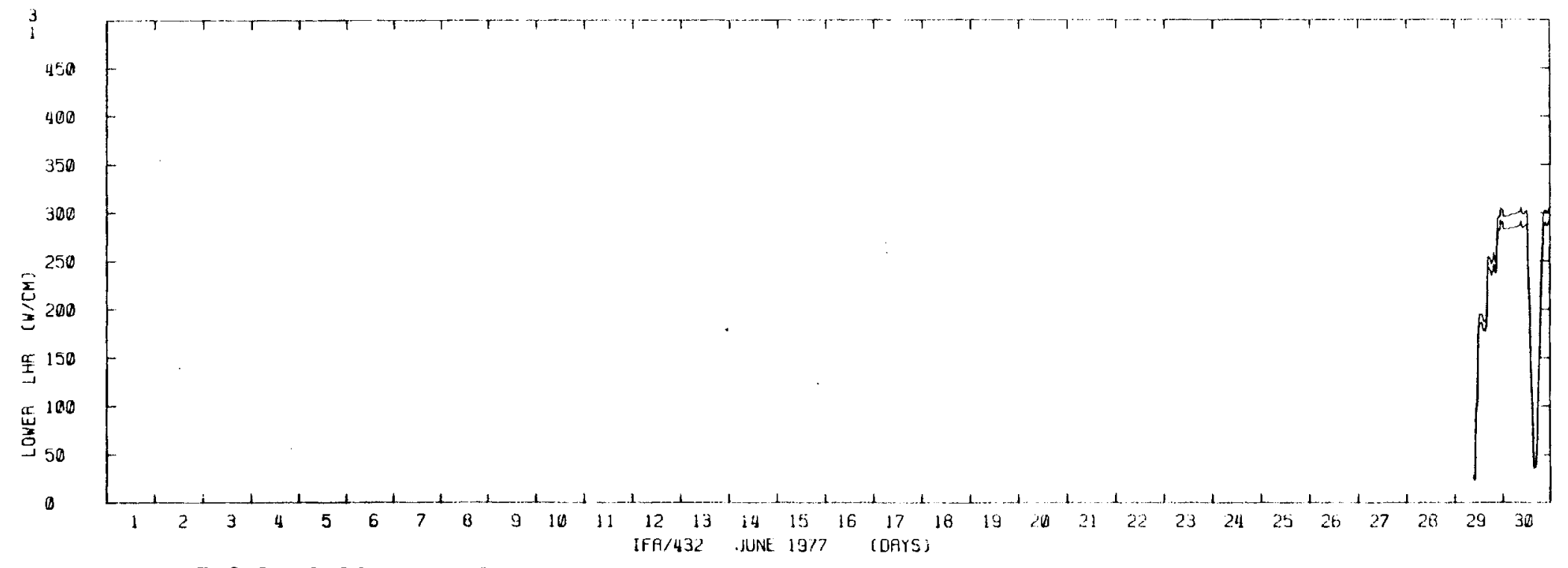

FIGURE 3.86. Local Linear Heat Ratings at Lower Thermocouple Locations for Rods 1,3 of IFA 432 - June 1977 


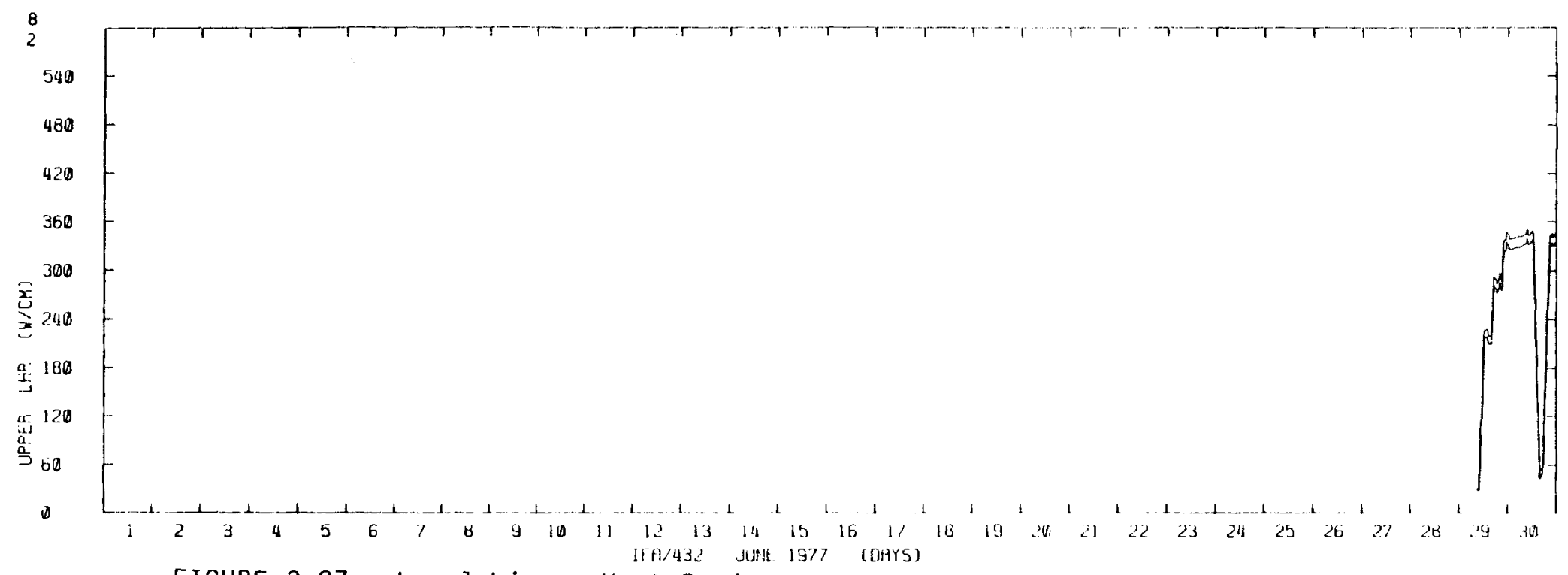

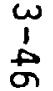

FIGURE 3.87. Local Linear Heat Ratings at Upper Thermocouple Locations for Rods 2,8 of IFA 432 - June 1977

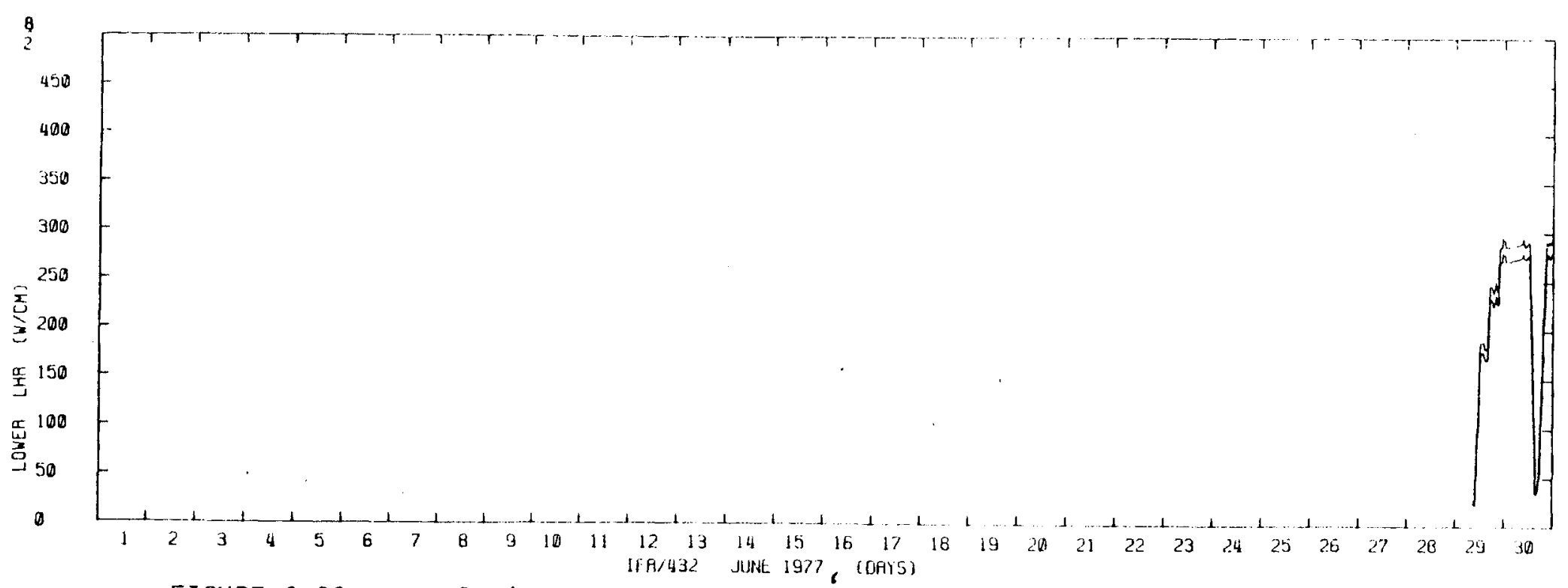

FIGURE 3.88. Local Linear Heat Ratings at Lower Thermocouple Locations for Rods 2,8 of IFA 432 - June 1977 


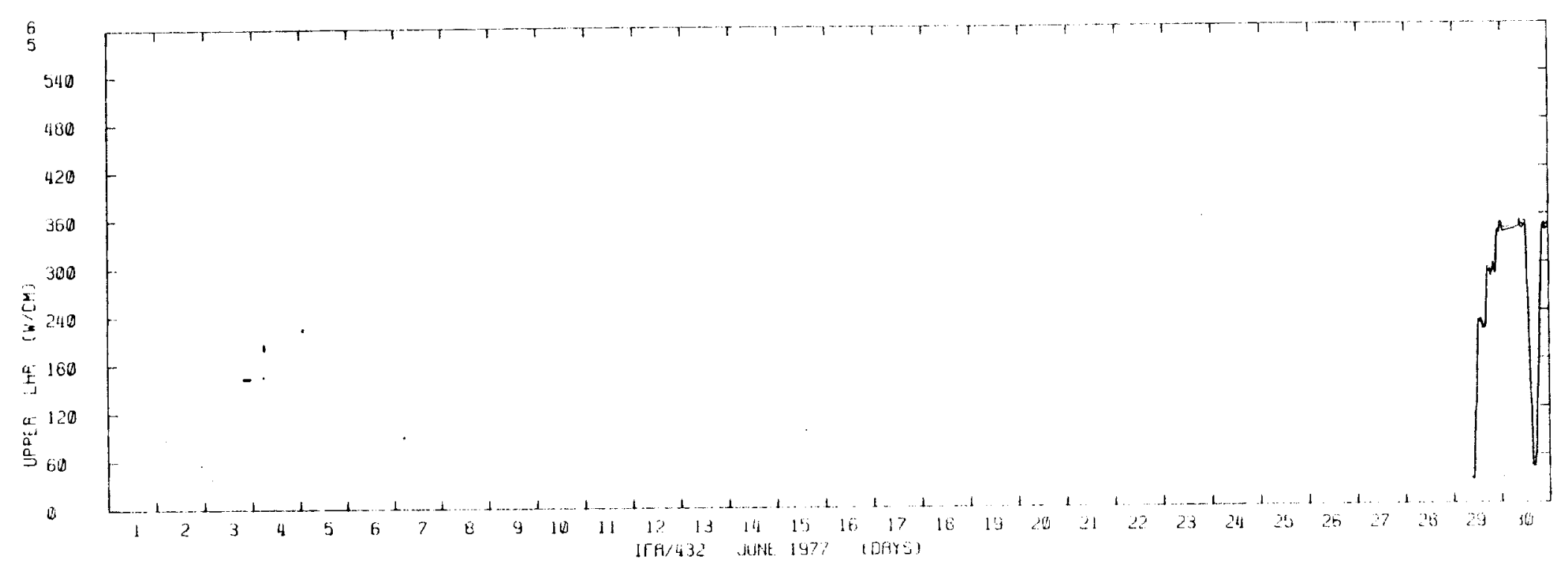

FIGURE 3.89. Local Linear Heat Ratings at Upper Thermocouple Locations for Rods 5,6 of IFA 432 - June 1977

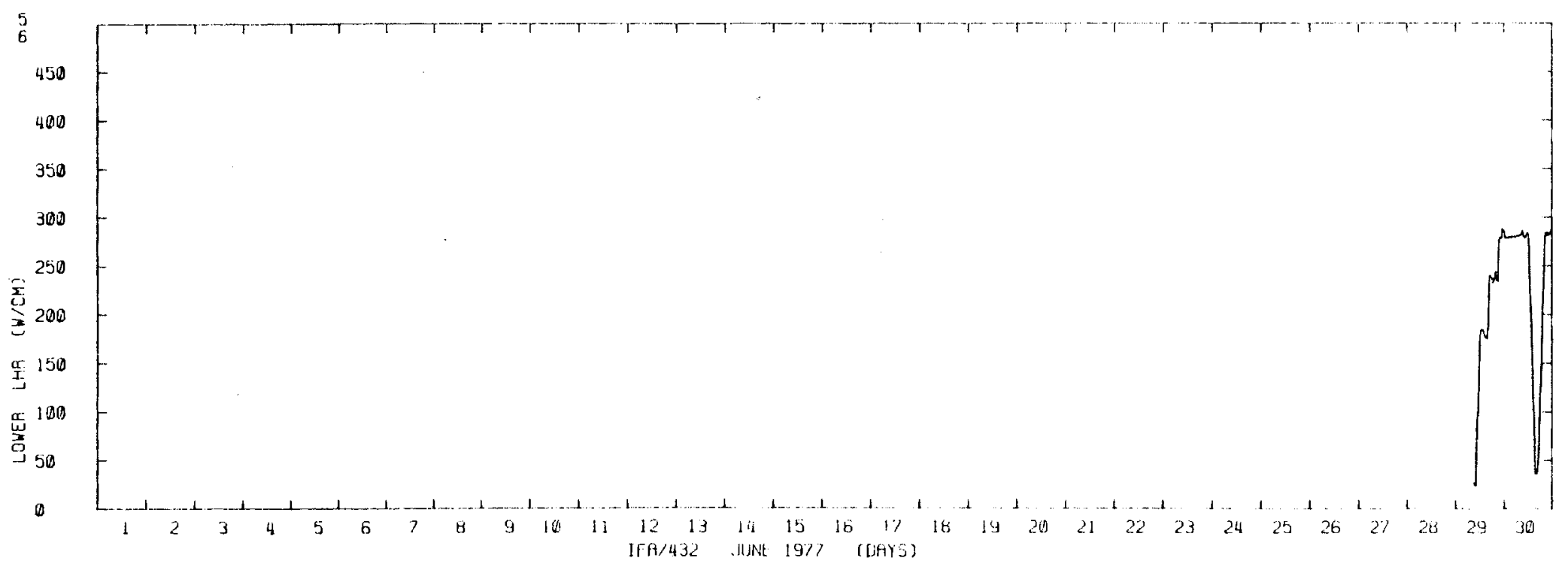

FIGURE 3.90. Local Linear Heat Ratings at Lower Thermocouple Locations for Rods 5,6 of IFA 432 - June 1977 


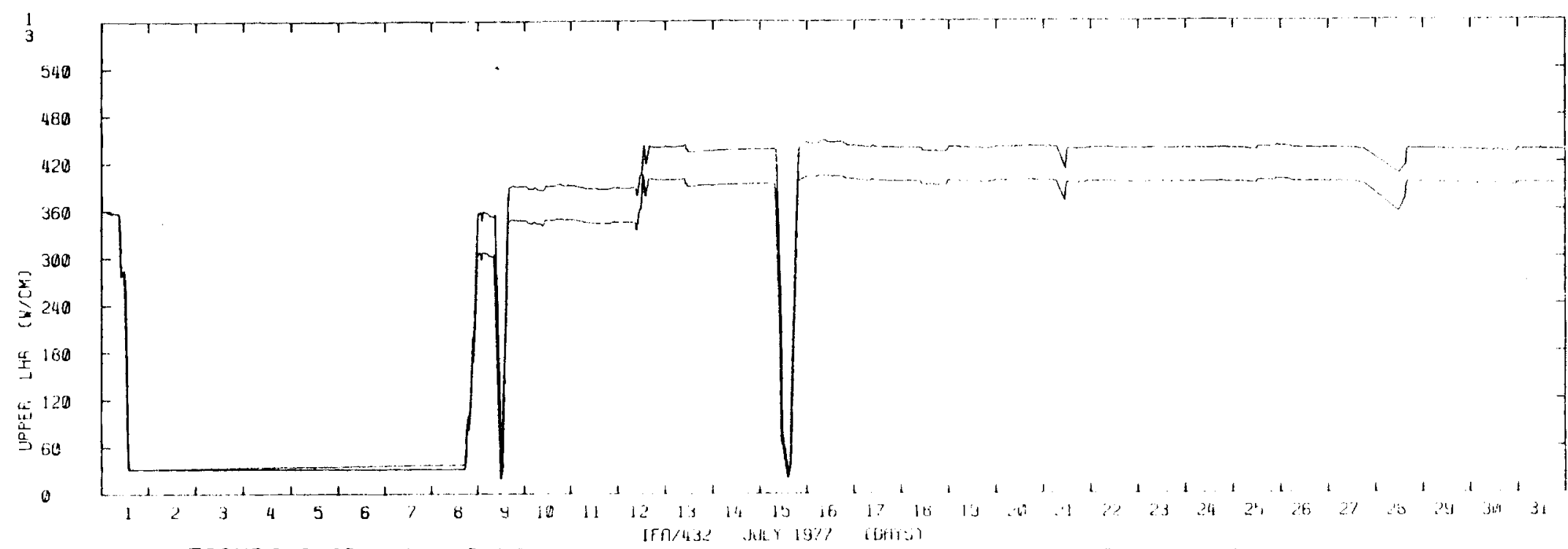

FIGURE 3.91. Local Linear Heat Ratings at Upper Thermocouple Locations for Rods 1,3 of IFA 432 - July 1977

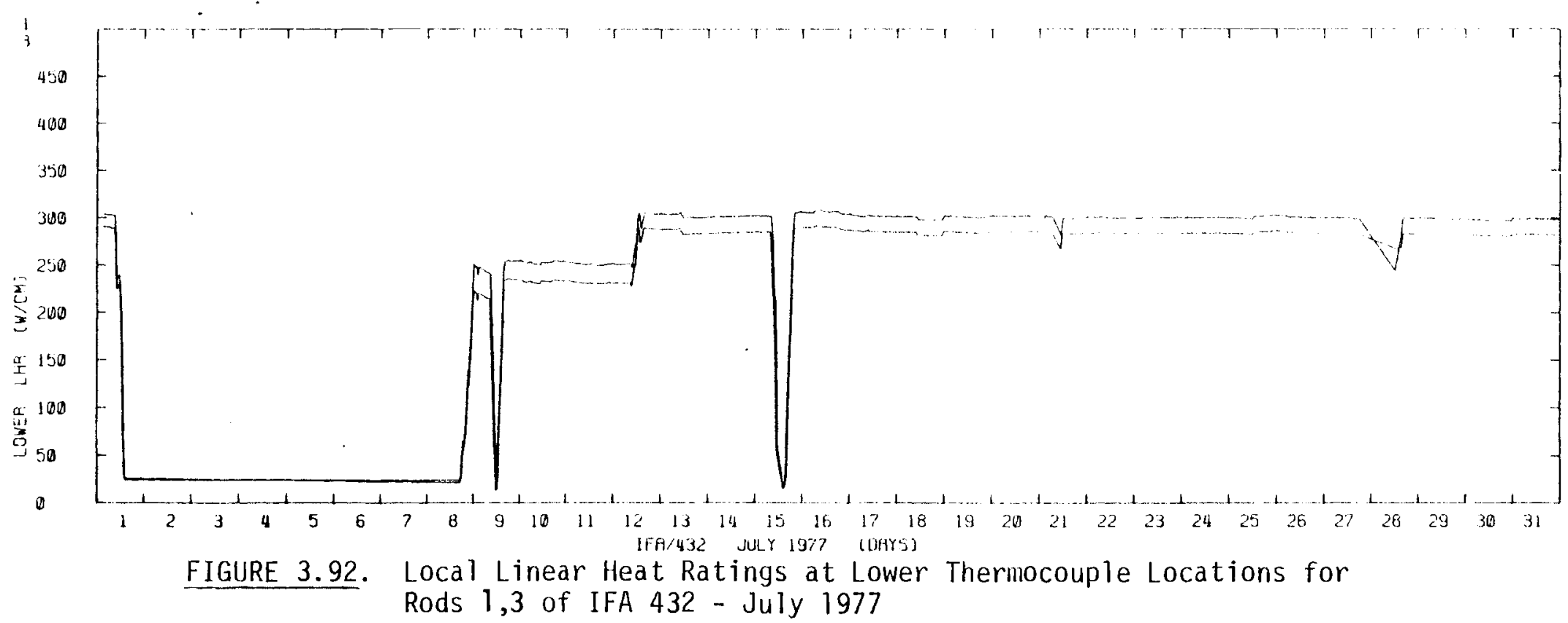




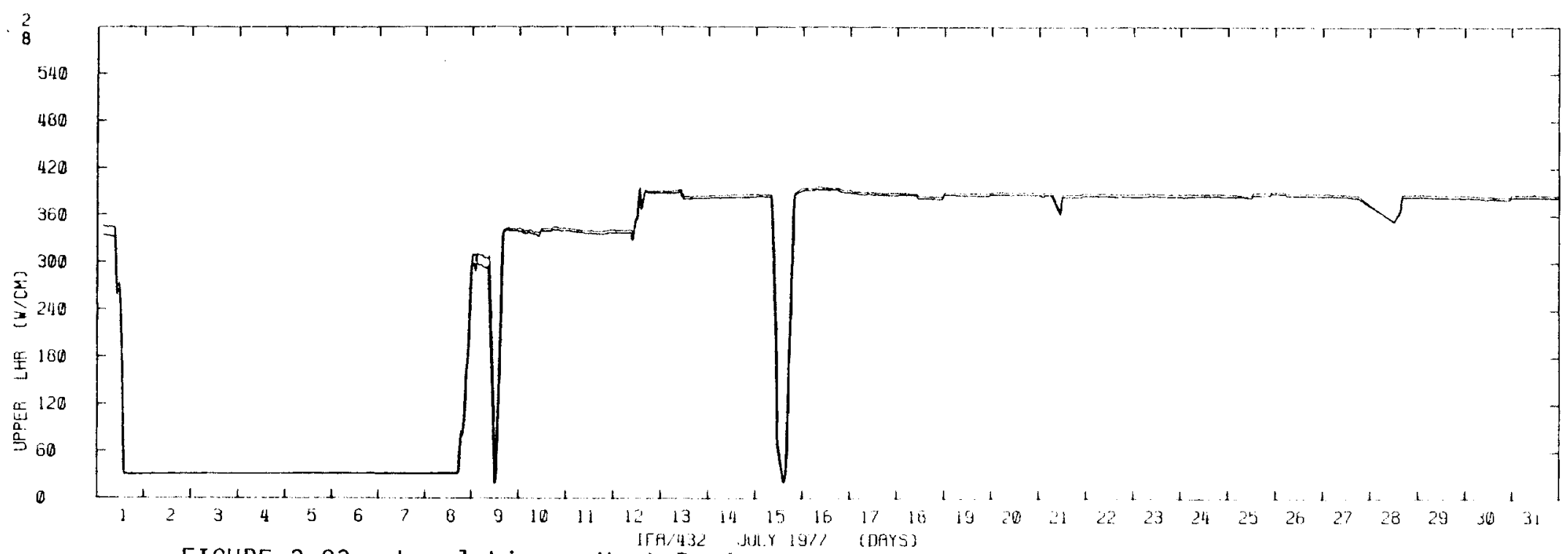

FIGURE 3.93. Local Linear Heat Ratings at Upper Thermocouple Locations for Rods 2,8 vi IFA 432 - July 1977

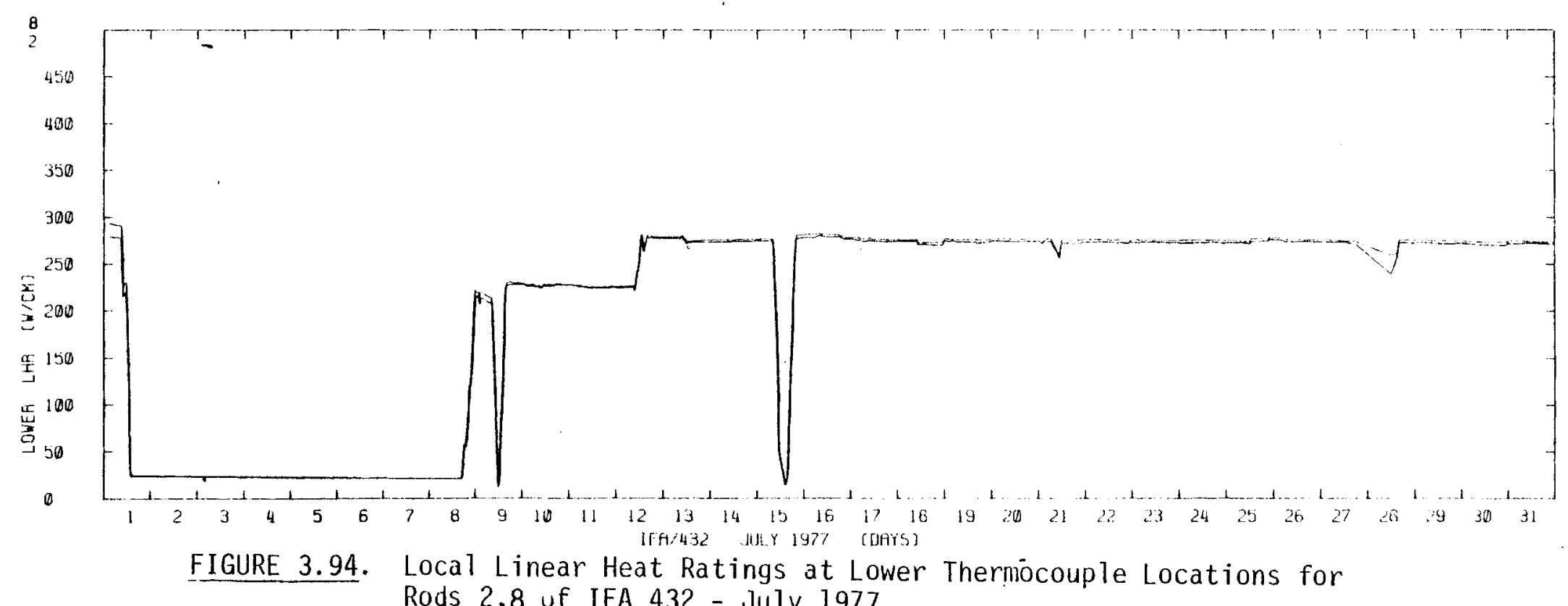




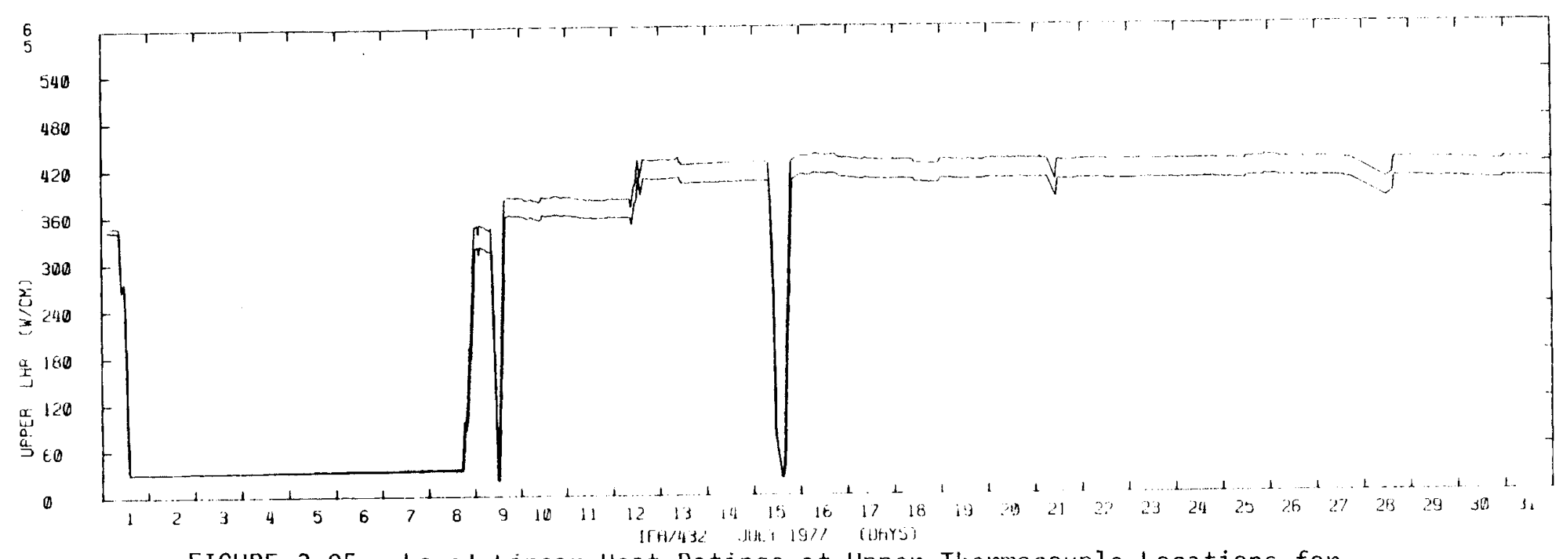

$w$
1
0

FIGURE 3.95. Local Linear Heat Ratings at Upper Thermocouple Locations for Rods 5,6 of IFA 432 - July 1977

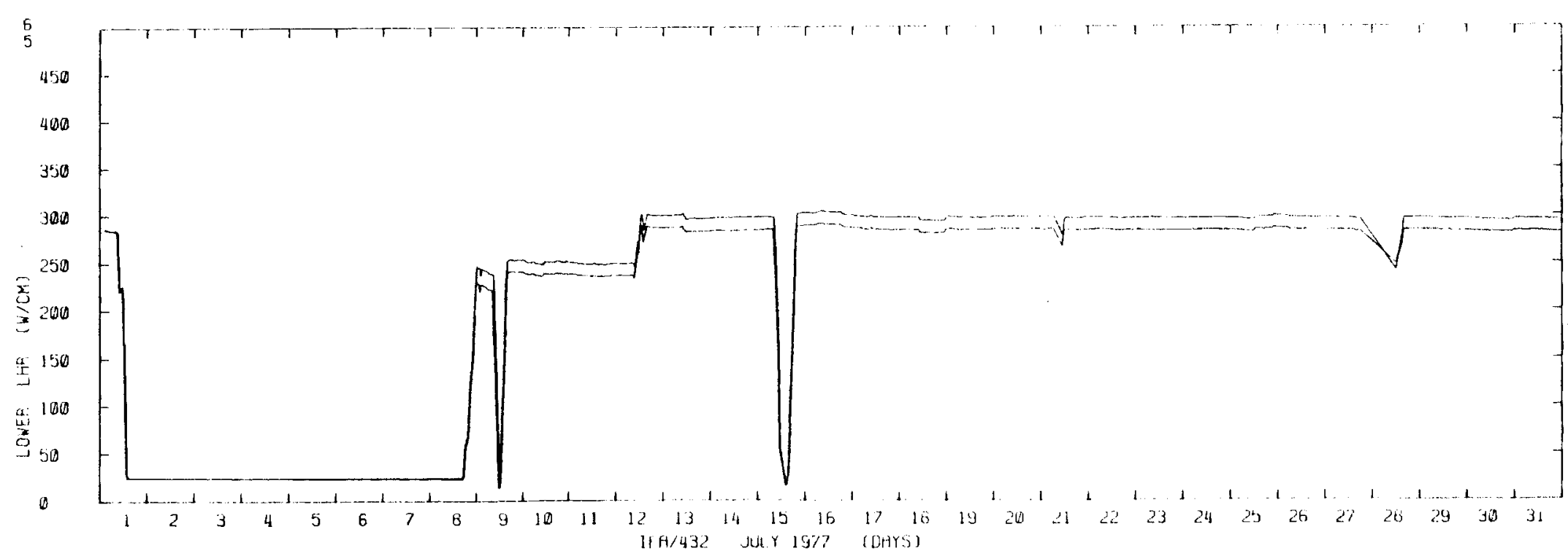

FIGURE 3.96. Local Linear Heat Ratings at Lower Thermocouple Locations for Rods 5,6 of IFA 432 - July 1977 


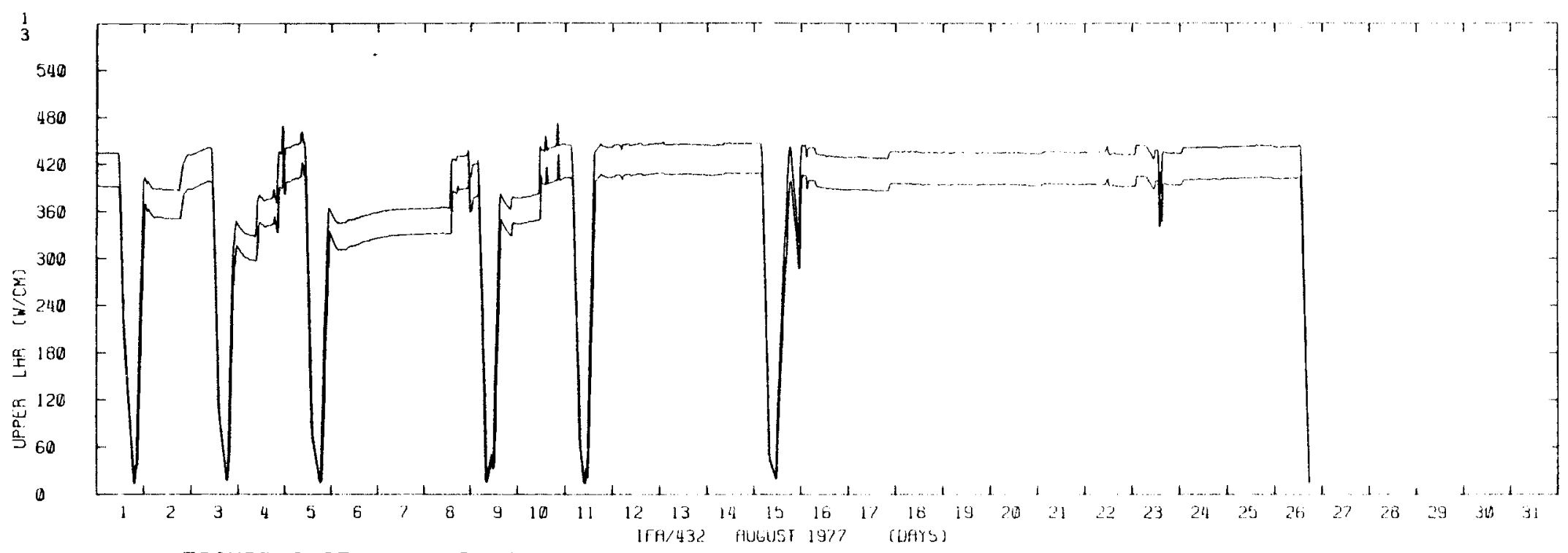

$\omega$
1

FIGURE 3.97. Local I inear Heat Ratings at Upper Thermocouple Locations for Rods 1,3 of IFA 432 - August 1977

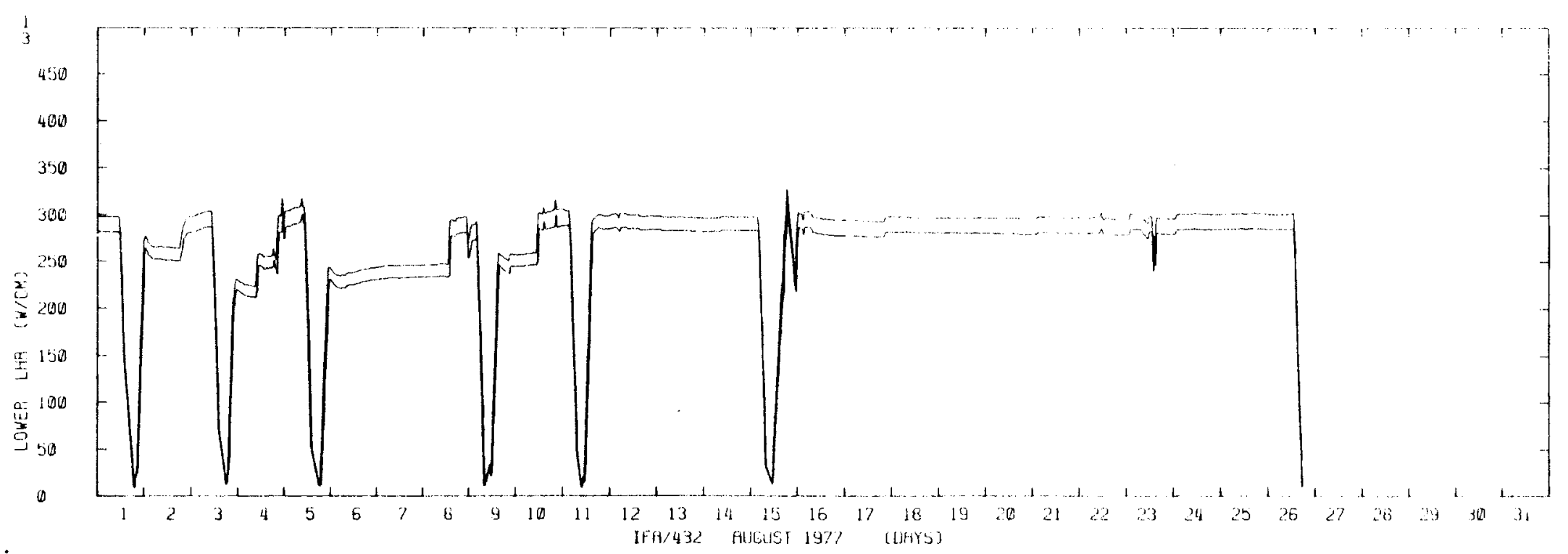

FIGURE 3.98. Local Linear Heat Ratings at Lower Thermocouple Locations for Rods 1,3 of IFA 432 - August 1977 


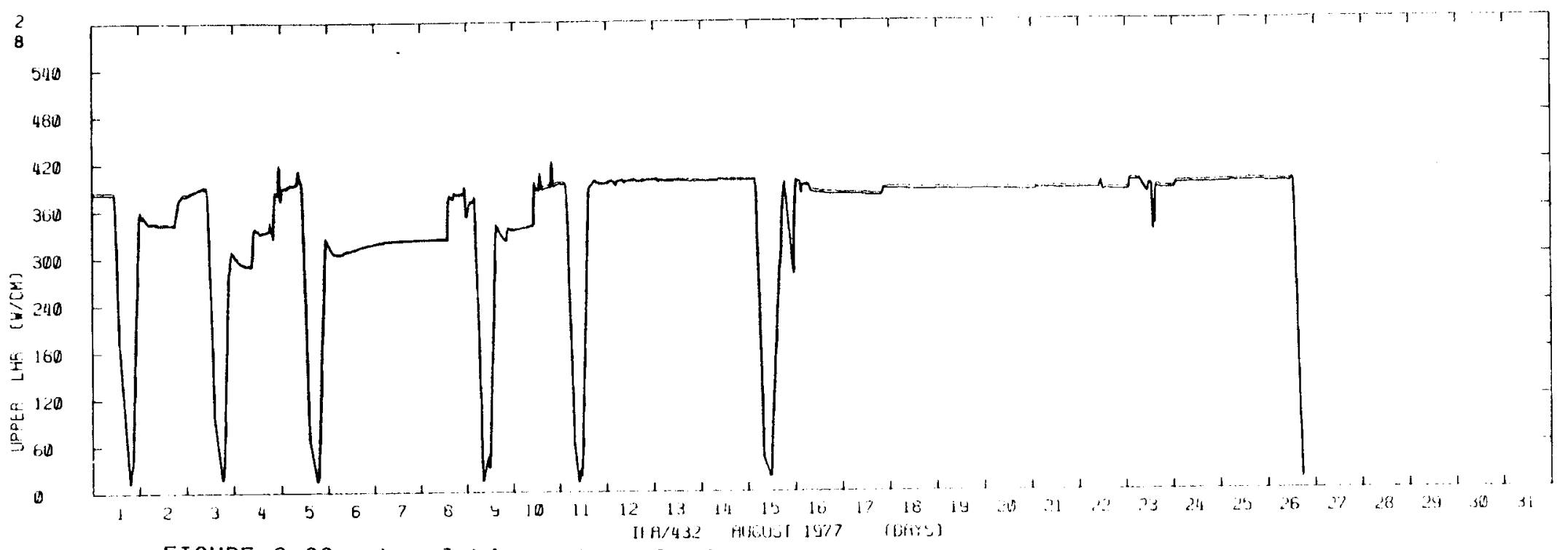

W

FIGURE 3.99. Local Linear Heat Ratings at Upper Thermocouple Locations for Rods 2,8 of IFA 432 - August 1977

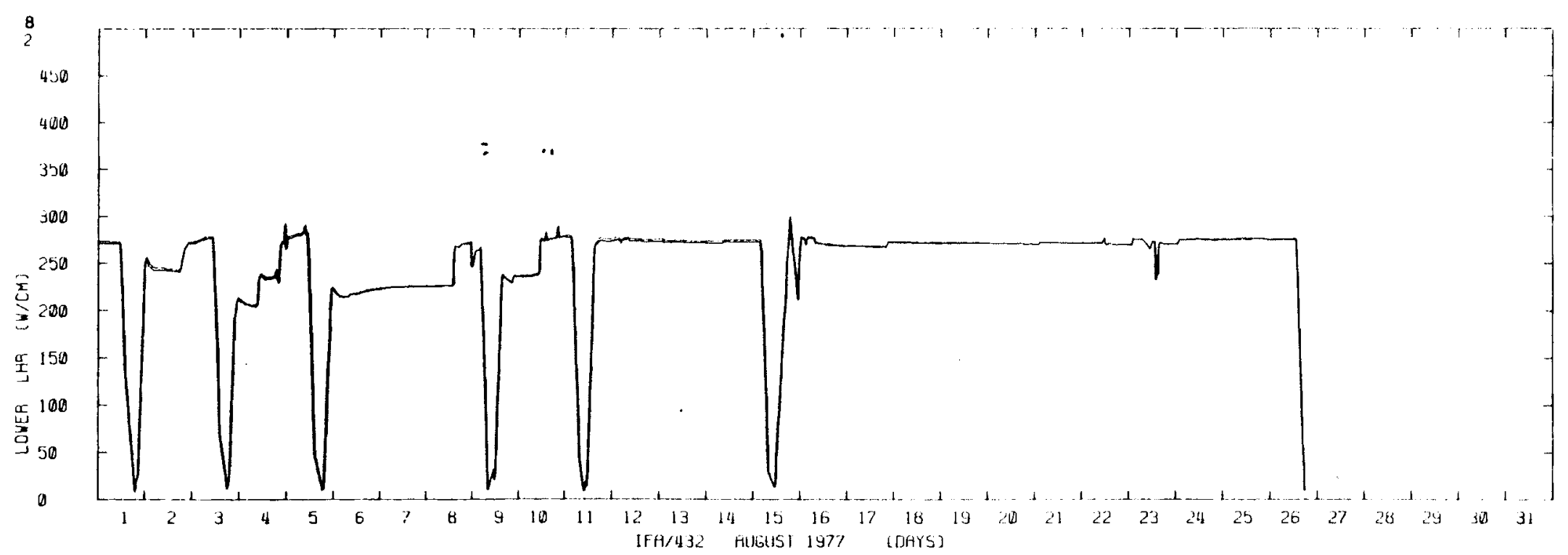

FIGURE 3.100. Local Linear Heat Ratings at Lower Thermocouple Locations for Rods 2,8 of IFA 432 - August 1977 


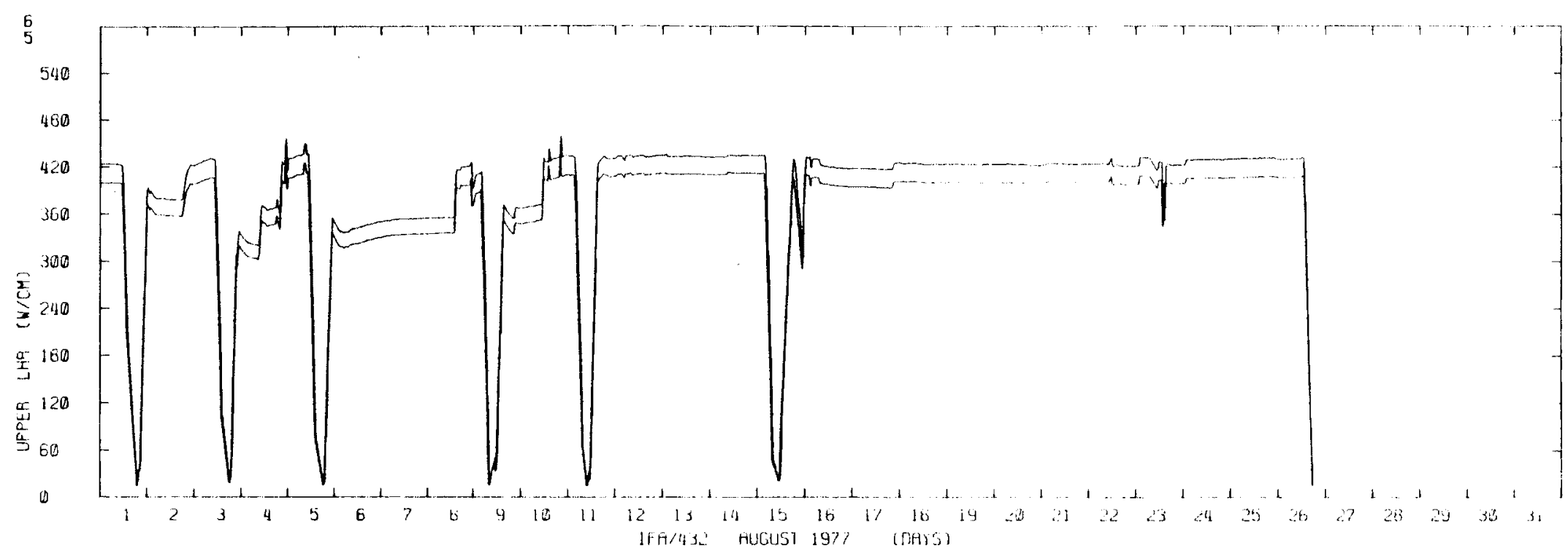

$\underset{\omega}{\omega}$

FIGURE 3.101. Local Linear Heat Ratings at Upper Thermocouple Locations for Rods 5,6 of IFA 432 - August 1977

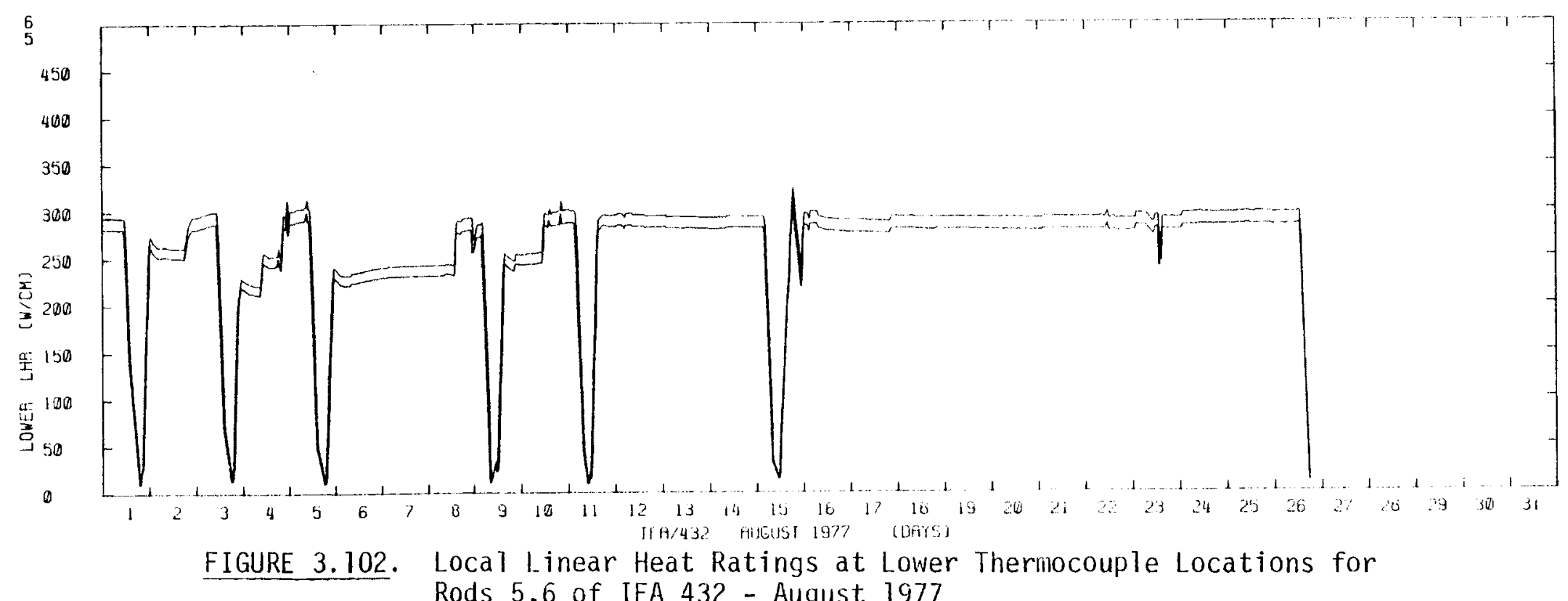




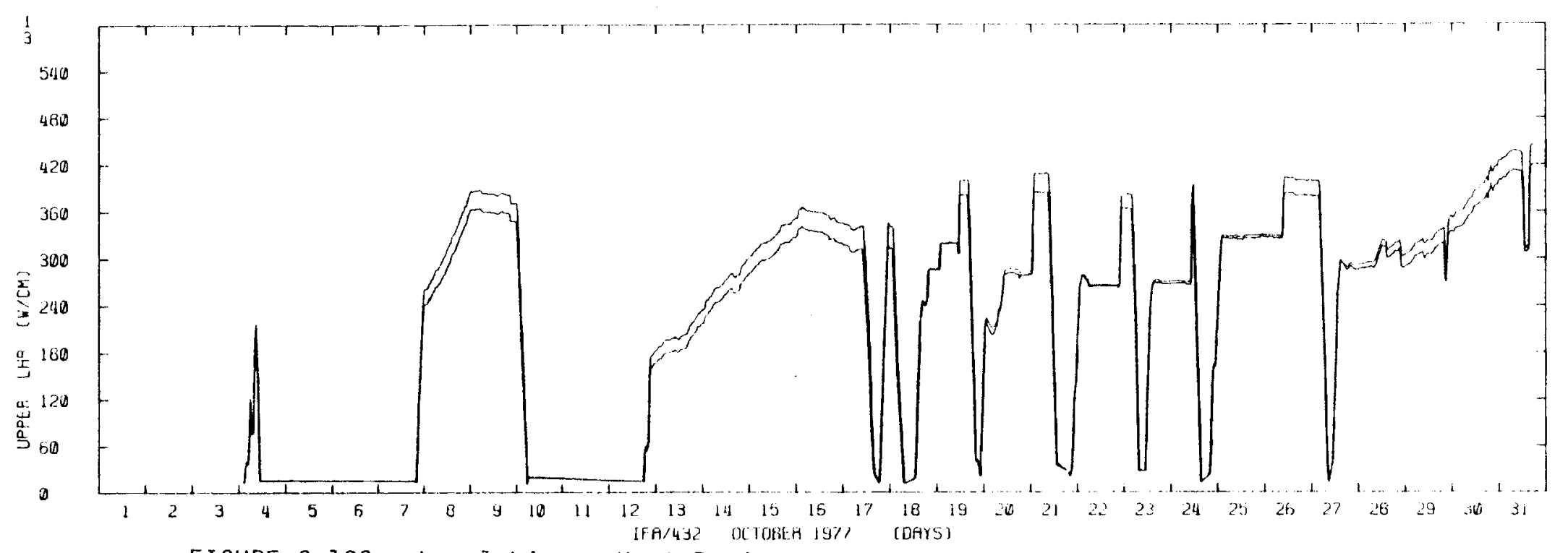

$\underset{1}{1}$

FIGURE 3.103. Local Linear Heat Ratings at Upper Thermocouple Locations for Rods 1,3 of IFA 432 - October 1977

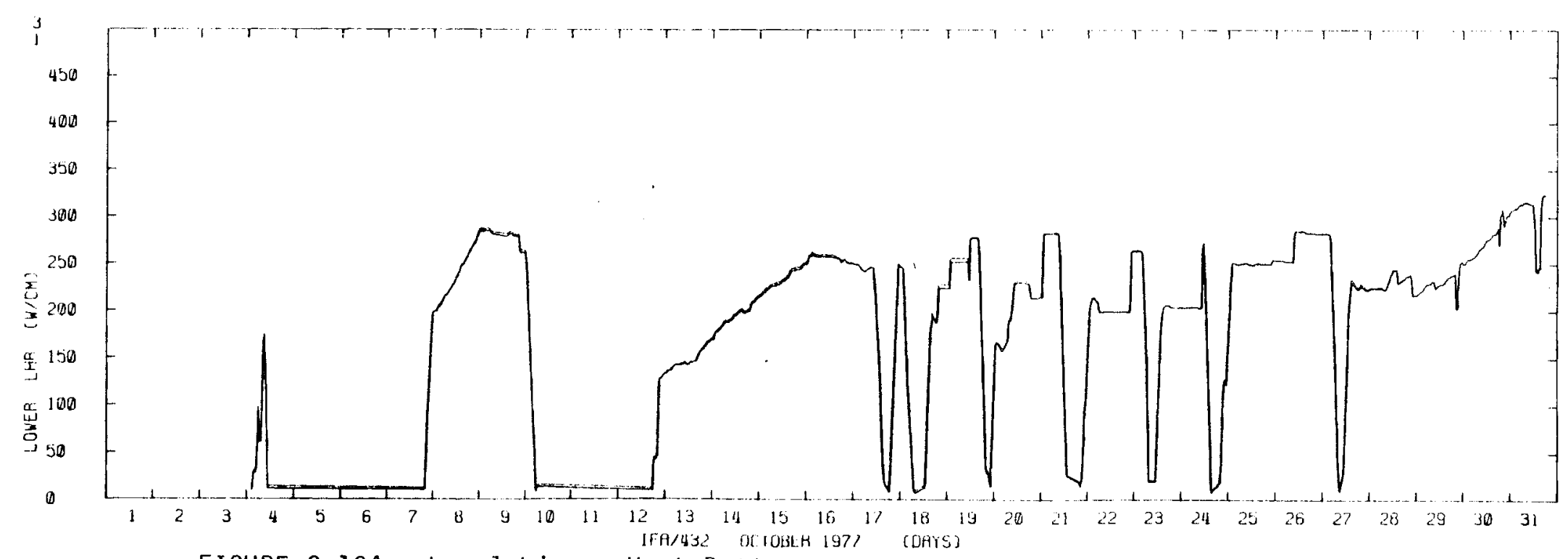

FIGURE 3.104. Local Linear Heat Ratings at Lower Thermocouple Locations for Rods 1,3 of IFA 432 - October 1977 


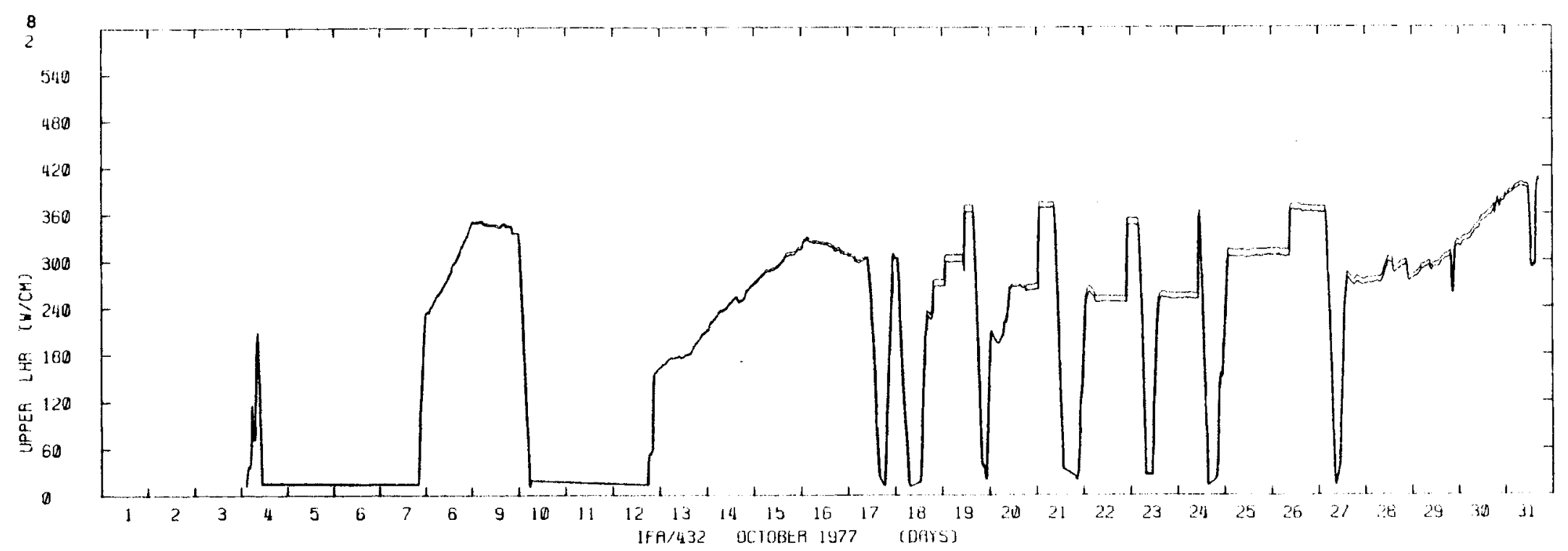

$\omega$
$\vdots$
Gi

FIGURE 3.105. Local Linear Heat Ratings at Upper Thermocouple Locations for Rods 2,8 of IFA 432 - October 1977

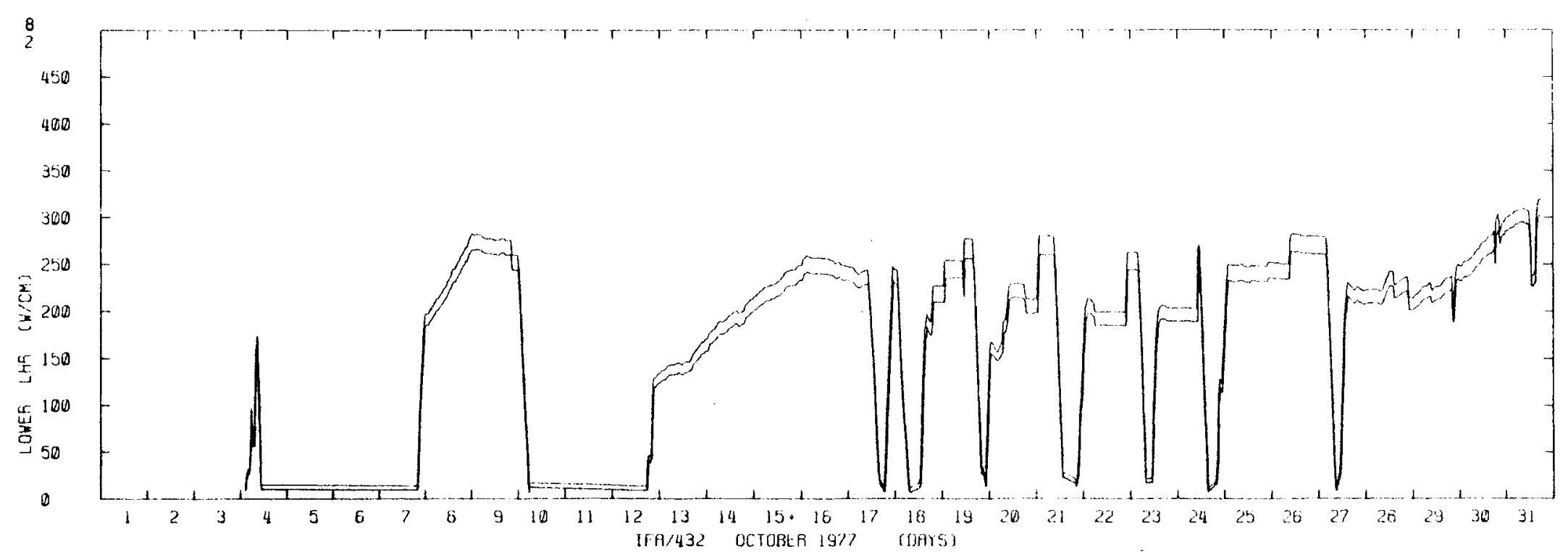

FIGURE 3.106. Local Linear Heat Ratings at Lower Thernocouple Locations for Rods 2,8 of IFA 432 - October 1977 


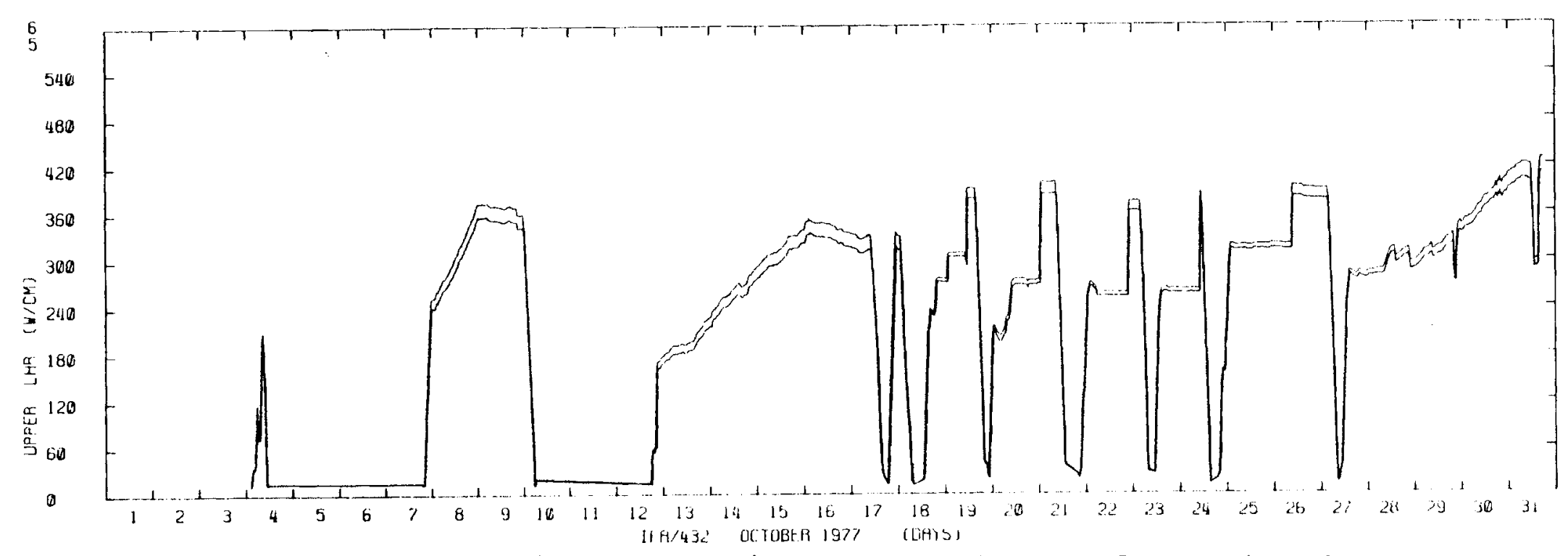

$w$
1
$o$

FIGURE 3.107. Local Linear Heat Ratings at Upper Thermocouple Locations for Rods 5,6 of IFA 432 - 0ctober 1977

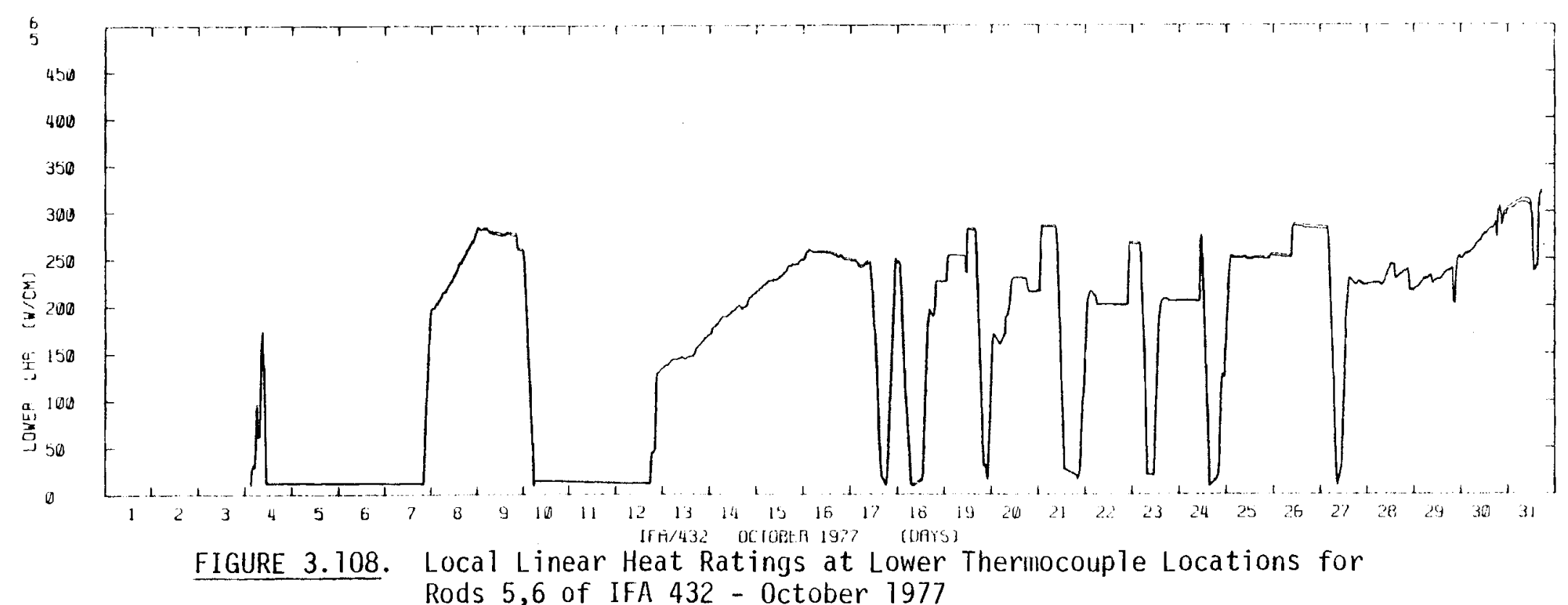




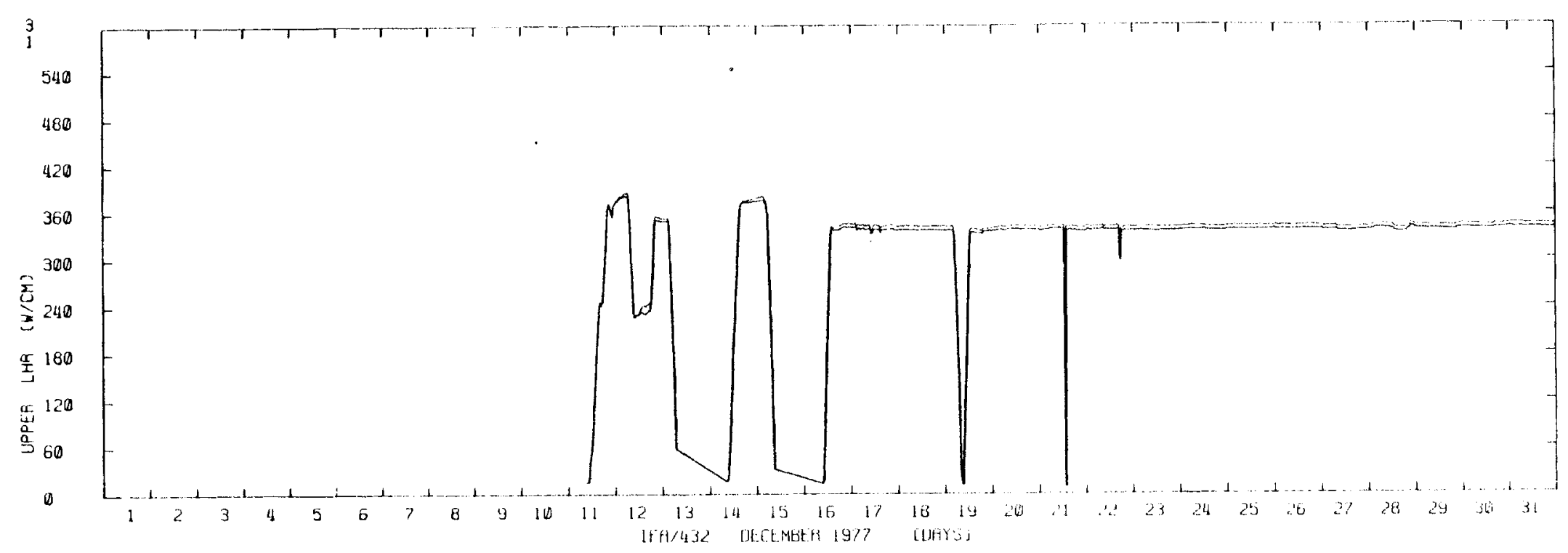

$\underset{w}{\omega}$

FIGURE 3.109. Local Linear Heat Ratings at Upper Thermocouple Locations for Rods 1,3 of IFA 432 - December 1977

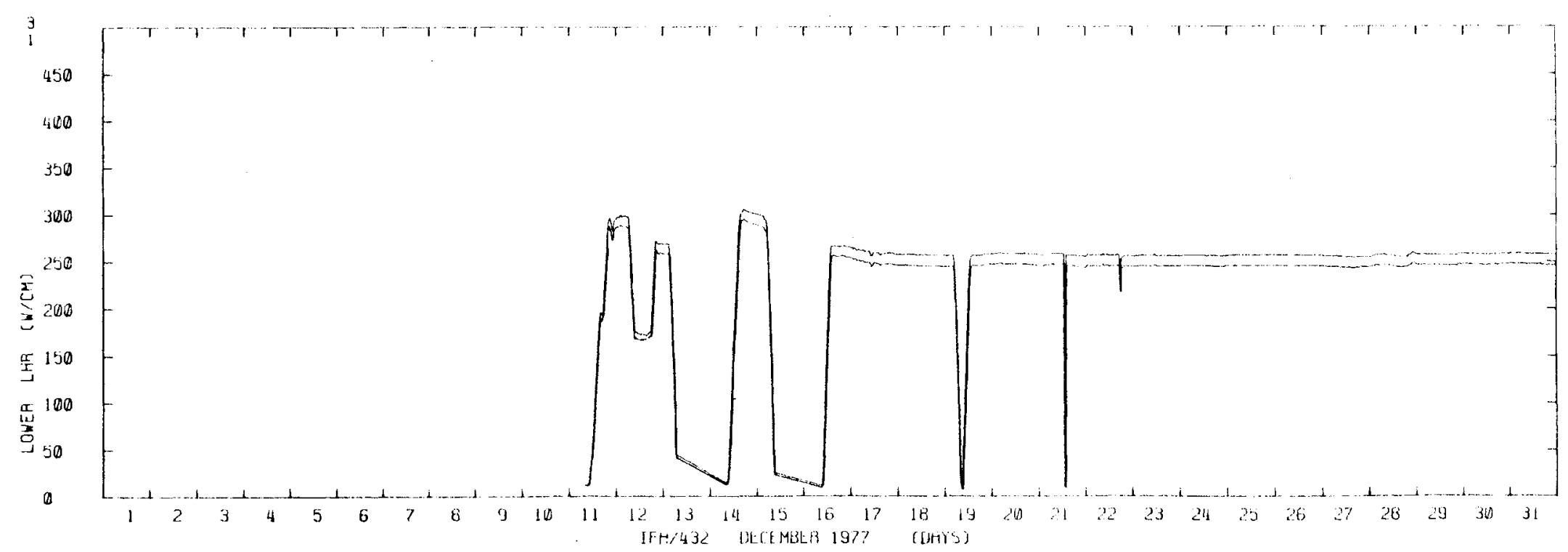

FIGURE 3.110. Local Linear Heat Ratings at Lower Thermocouple Locations for Rods 1,3 of IFA 432 - December 1977 


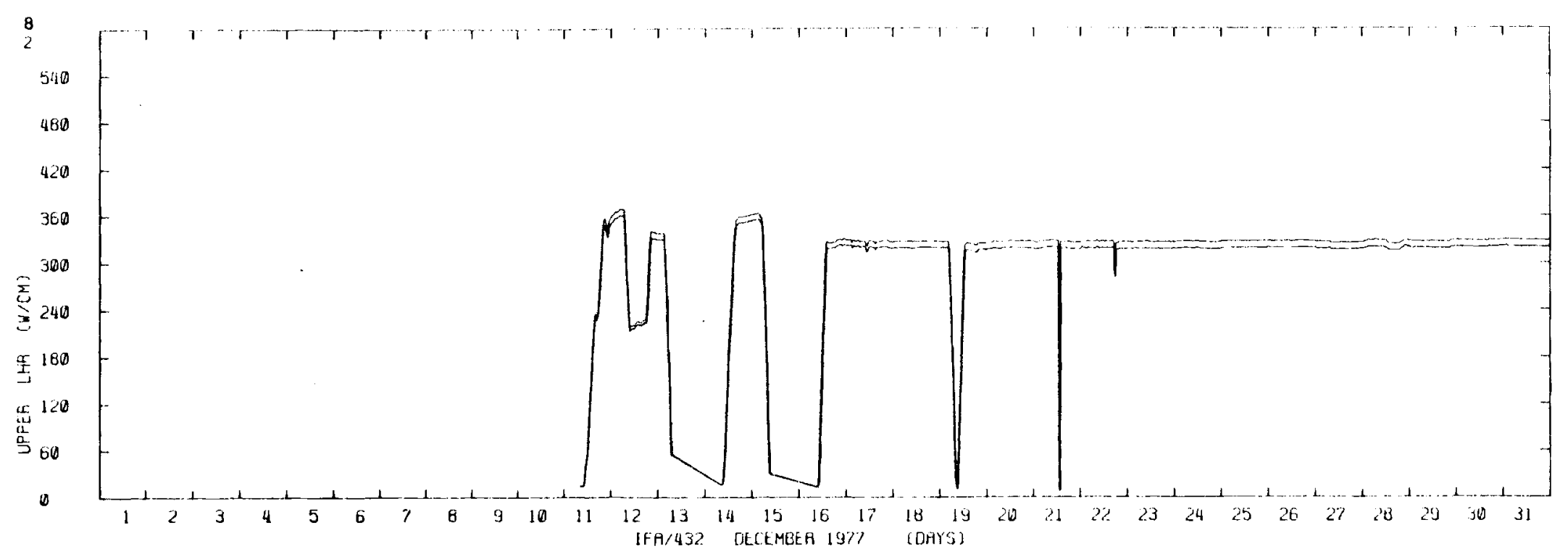

FIGURE 3.111. L.ocal Linear Heat Ratings at Upper Thermocouple Locations for Rods 2,8 of IFA 432 - December 1977

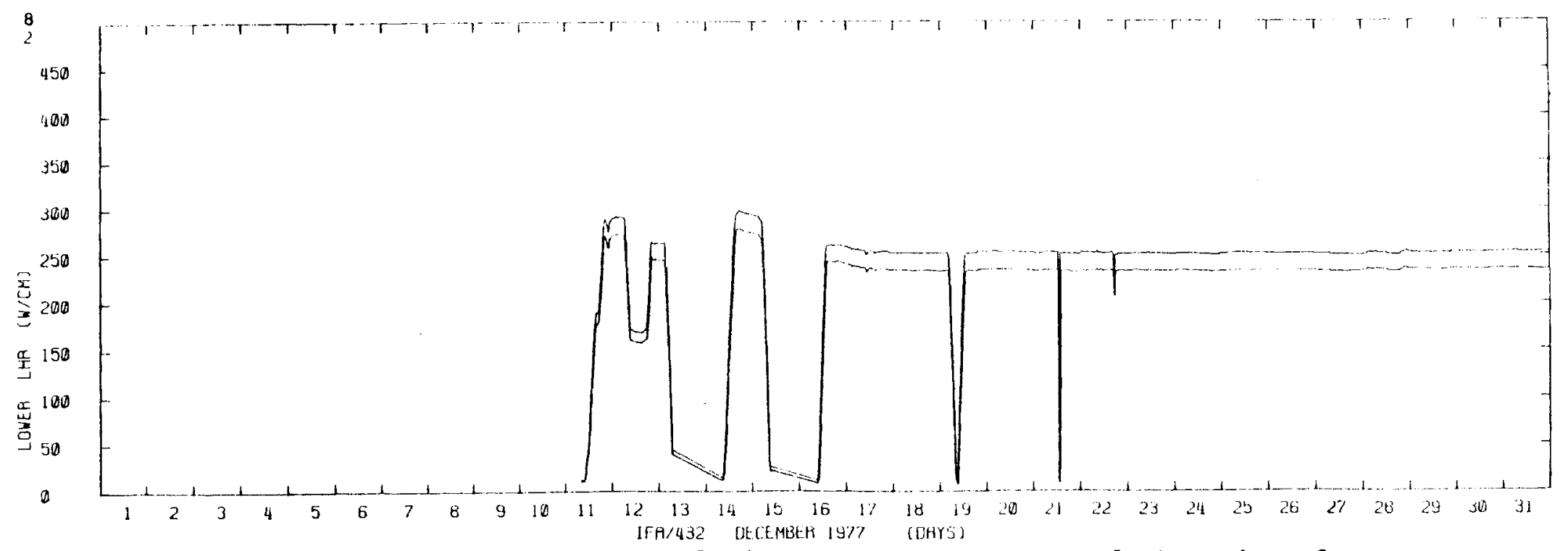

FIGURE 3.112. Local Linear Heat Ratings at Lower Thermocouple Locations for Rods 2,8 of IFA 432 - December 1977 


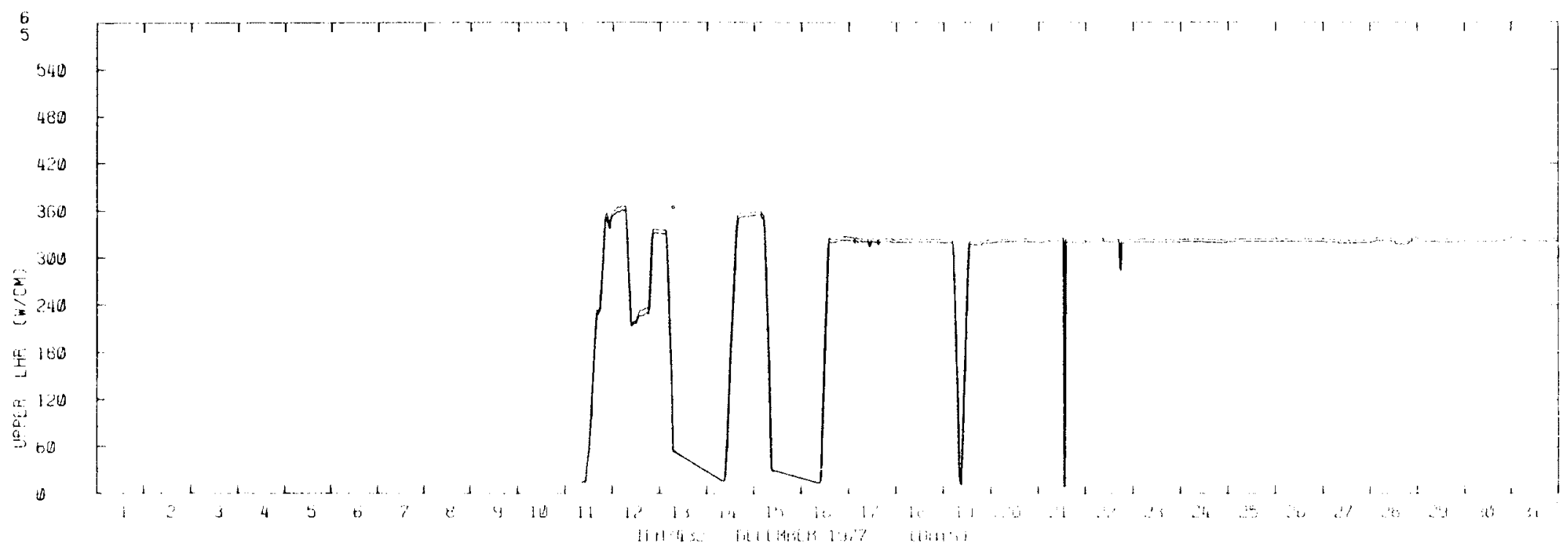

$\omega$
$\vdots$
$w$

FIGURE 3.113. Local Linear Heat Ratings at Upper Thermocouple Locations for Rods 5,6 of IFA 432 - December 1977

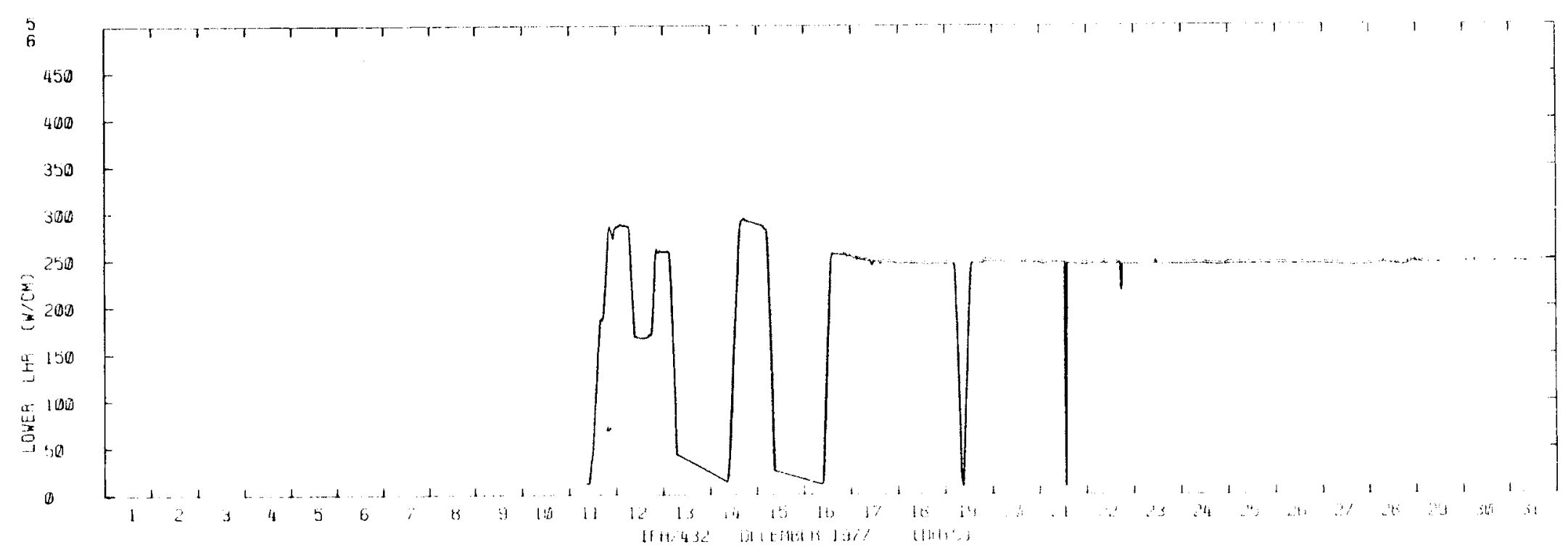

FIGURE 3.114. Local Linear Heat Ratings at Lower Theruocouple Locations for Rods 5,6 of IFA 432 - December 1977 


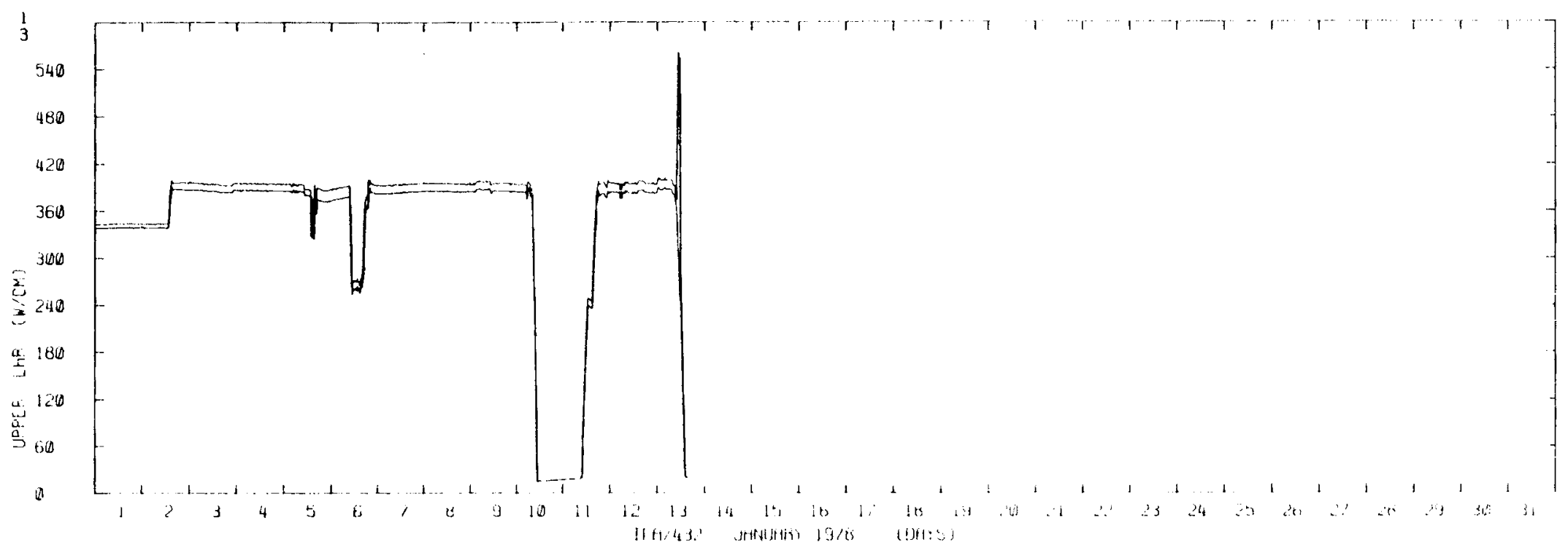

$\omega$
1
0
0

FIGURE 3.115. Local Linear Heat Ratings at Upper Thermocouple Locations for Rods 1,3 of IFA 432 - January 1978

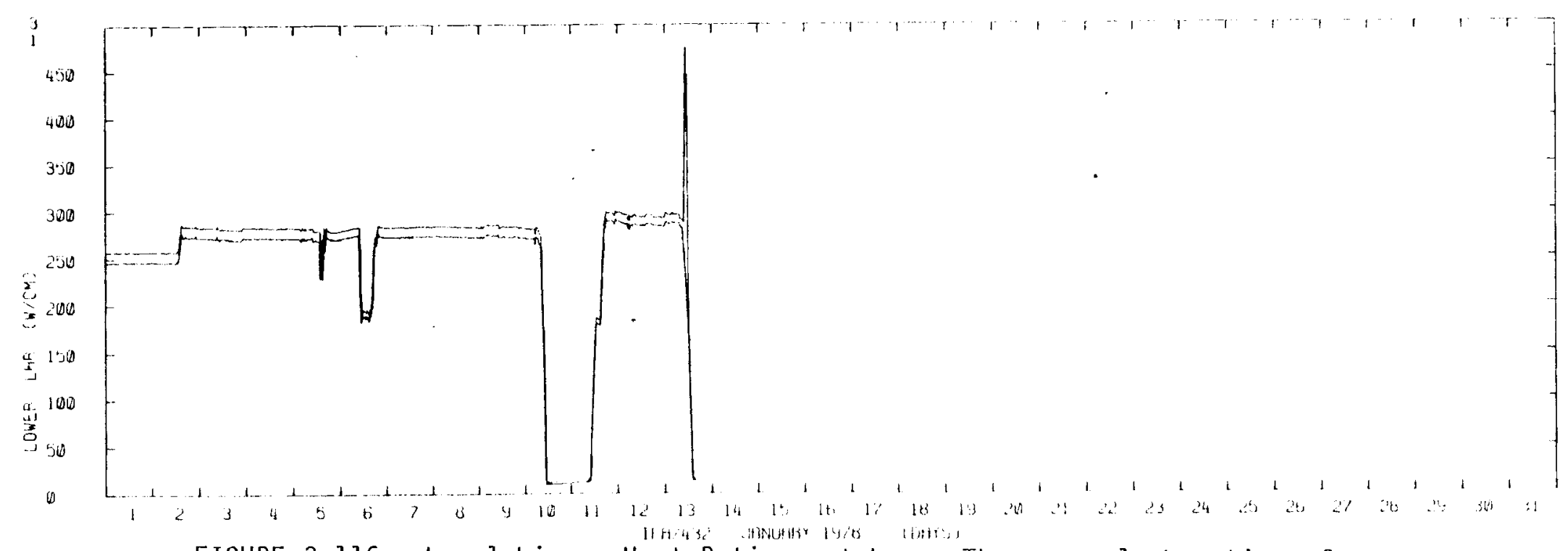

FIGURE 3.116. Local Linear Heat Ratings at Lower Thermocouple Locations for Rods 1,3 of IFA 432 - January 1978 


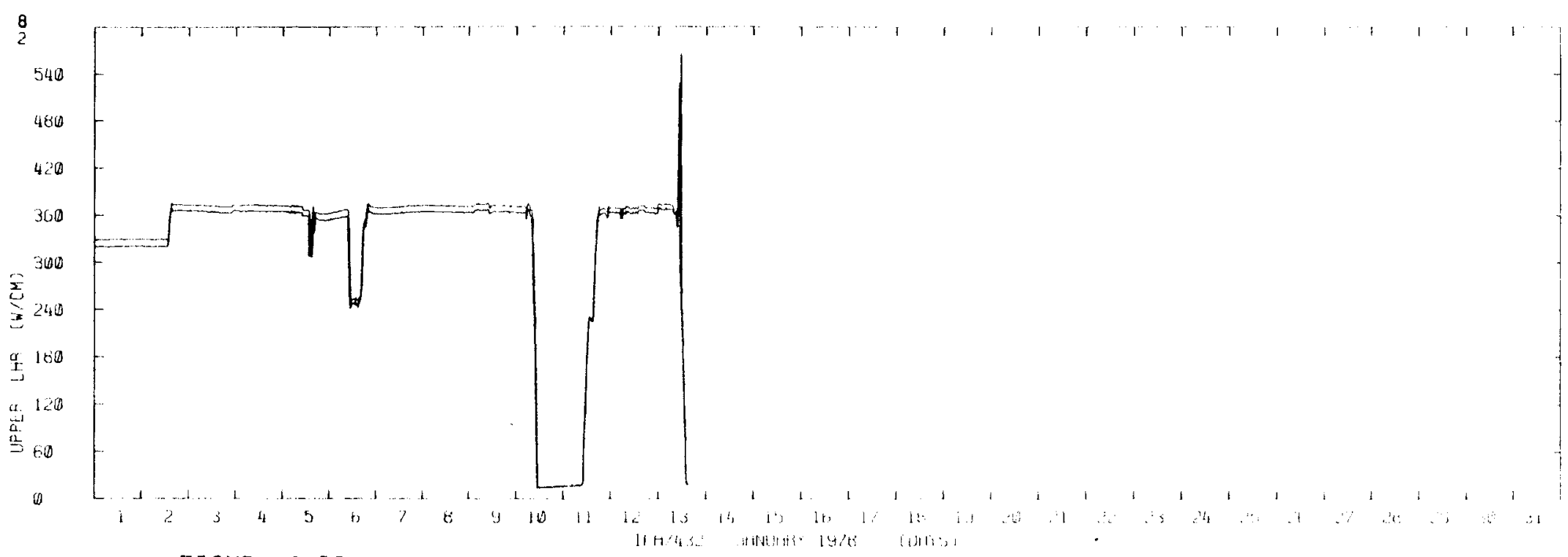

$\stackrel{\omega}{\stackrel{\omega}{S}}$

FIGURE 3.117. Local Linear Heat Ratings at Upper Thermocouple Locations for Rods 2,8 of IFA 432 - January 1978

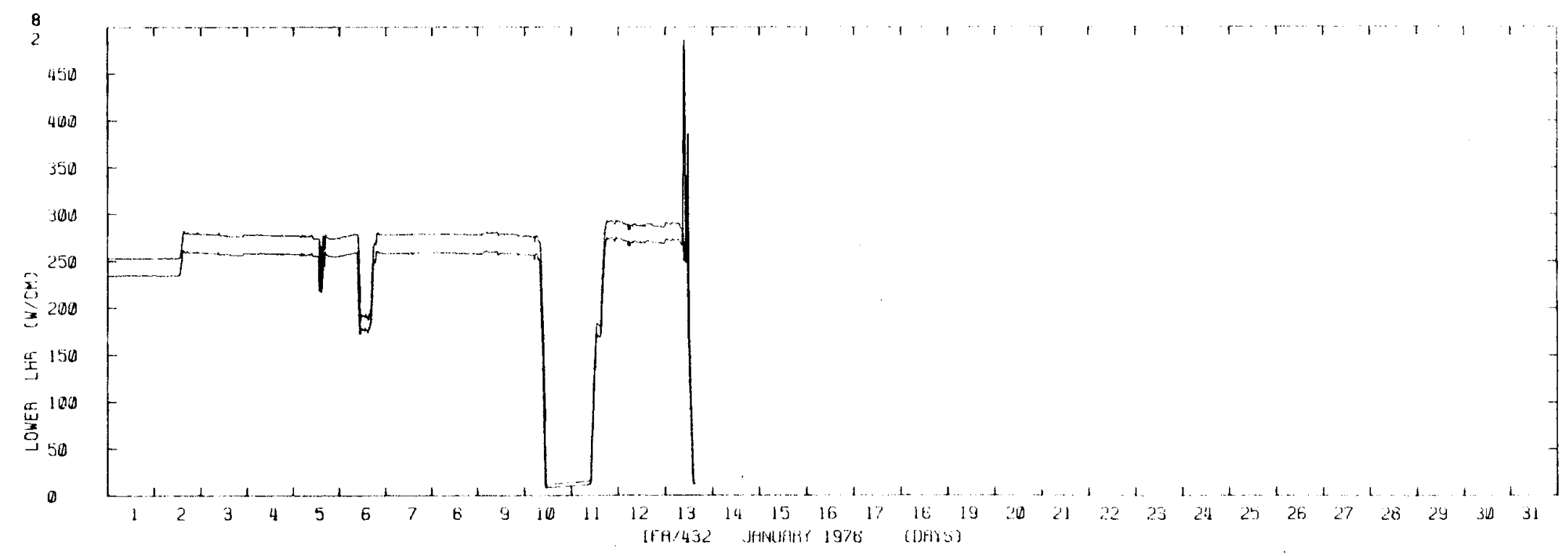

FIGURE 3.118. Local Linear Heat Ratings at Lower Thermocouple Locations for Rods 2,8 of IFA 432 - January 1978 


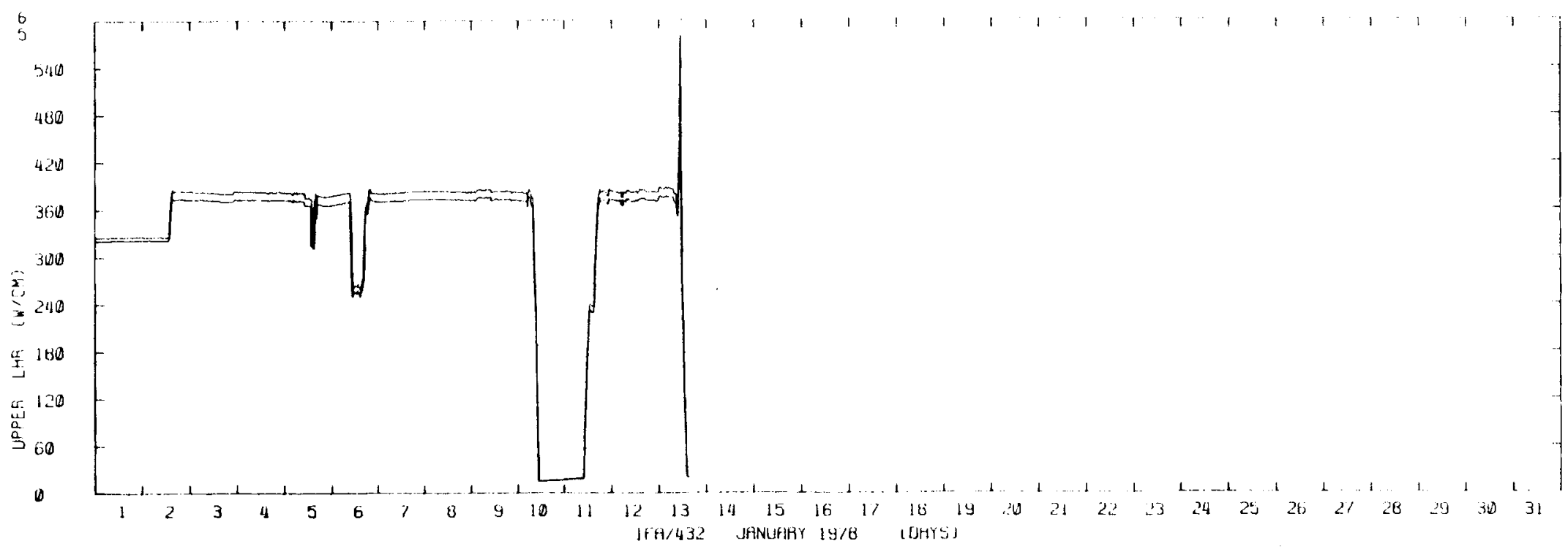

FIGURE 3.119. Local Linear Heat Ratings at Upper Thermocouple Locations for Rods 5,6 of IFA 432 - January 1978

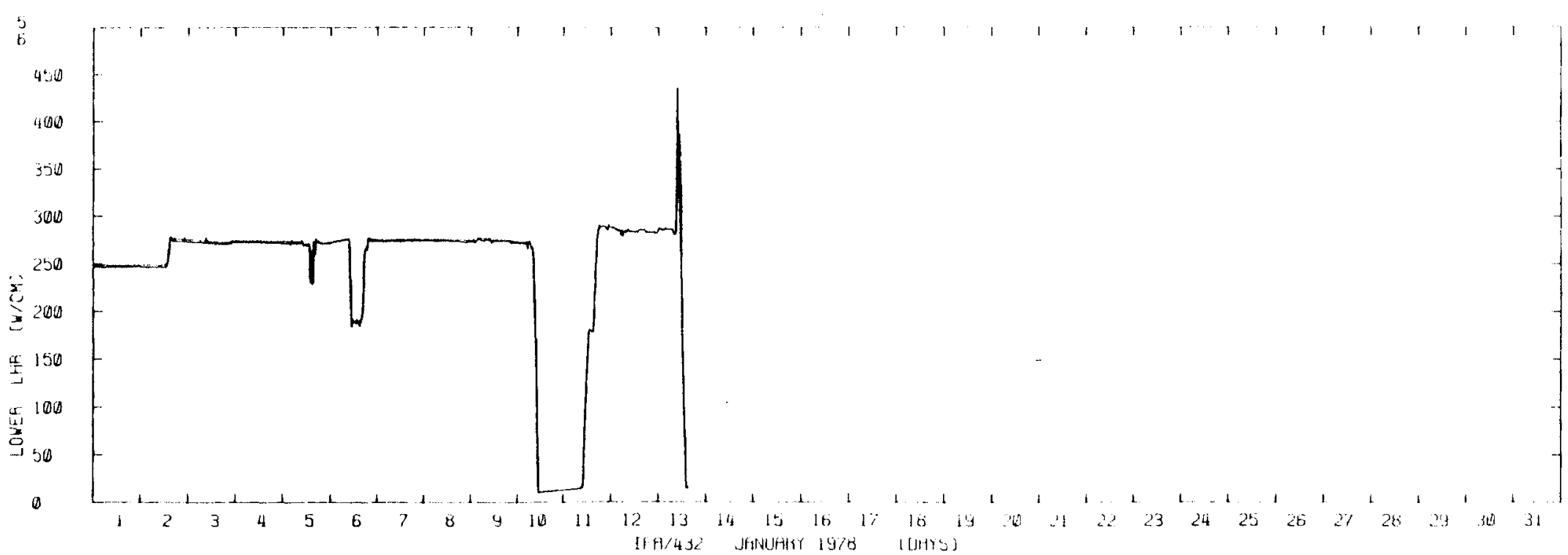

FIGURE 3.120. Local Linear Heat Ratings at Lower Thermocouple l.ocations for Rods 5,6 of IFA 432 - January 1978 


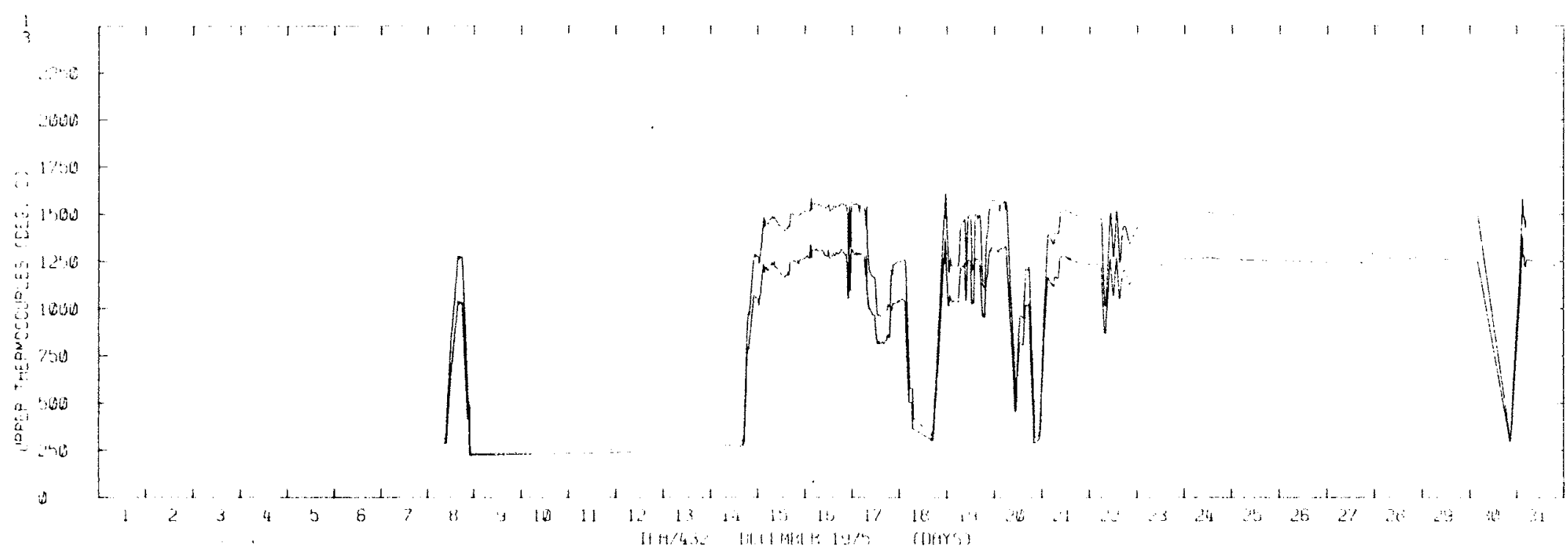

FIGURE 3.121. Upper Thermocouple Readings for Rods 1,3 of IFA 432 - December 1975

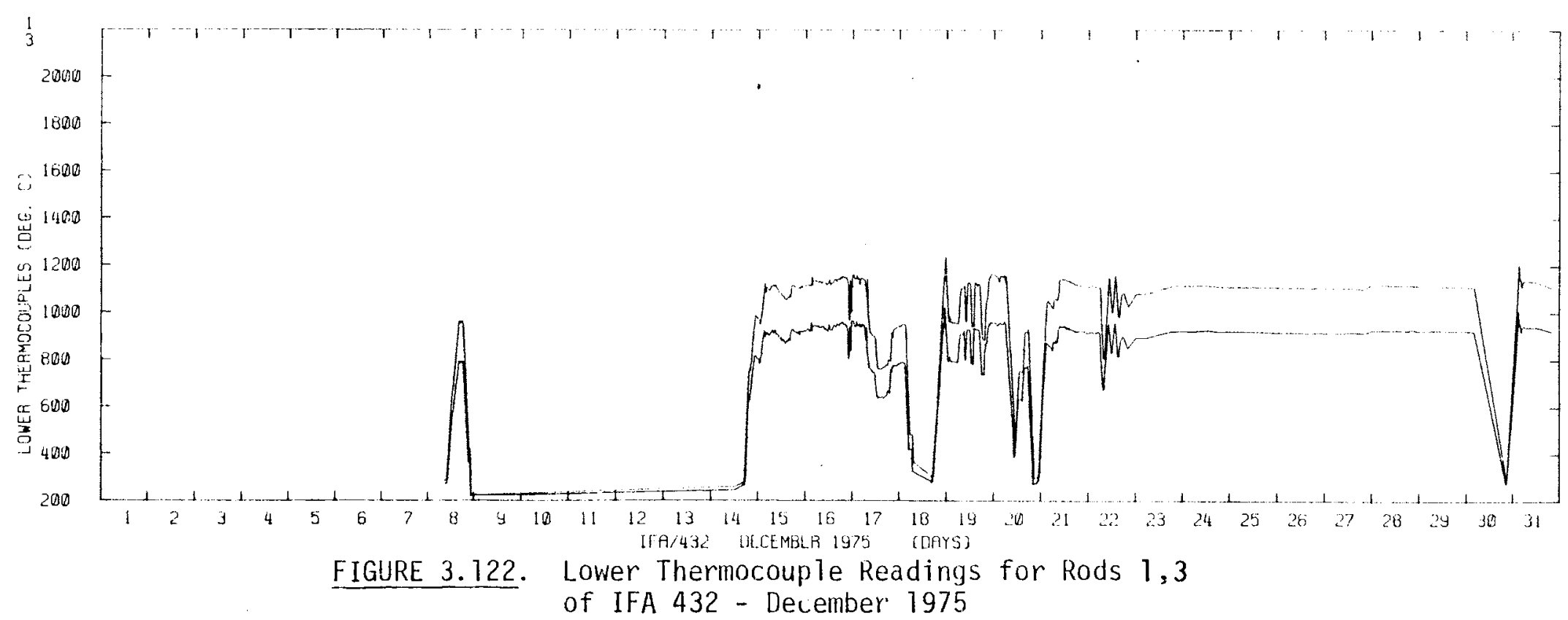




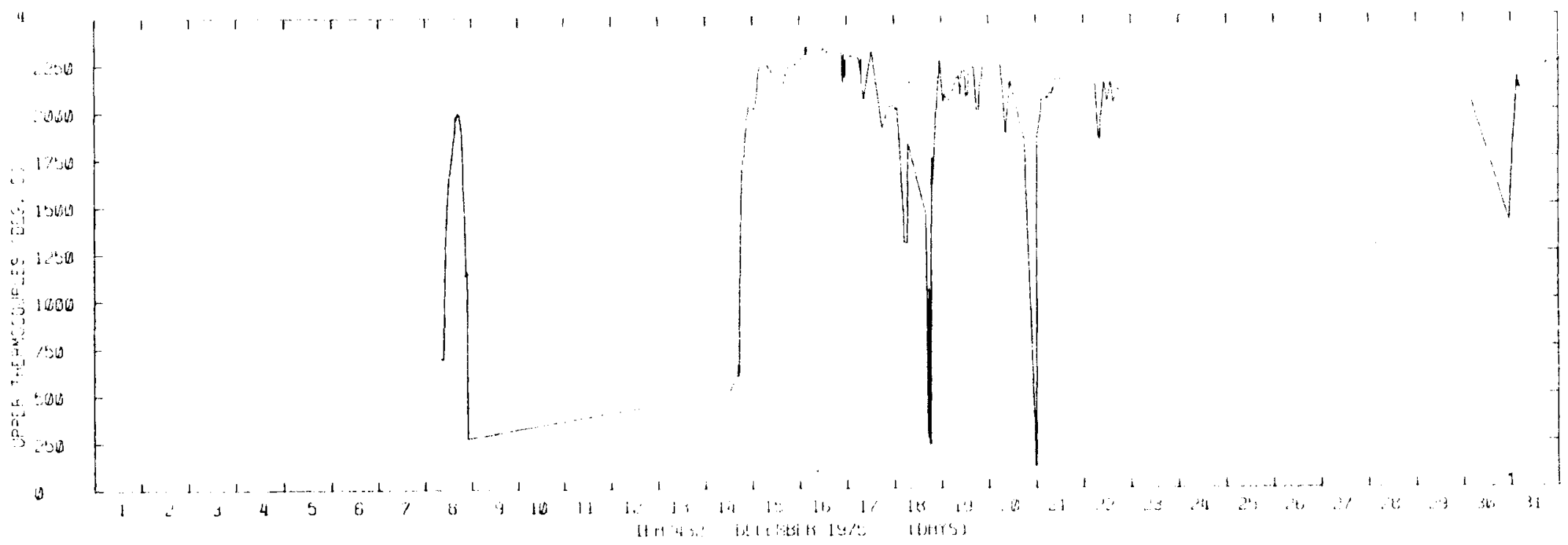

FIGURE 3.123. Upper Therliocouple Readings for Rod 4 of IFA 432 - December 1975

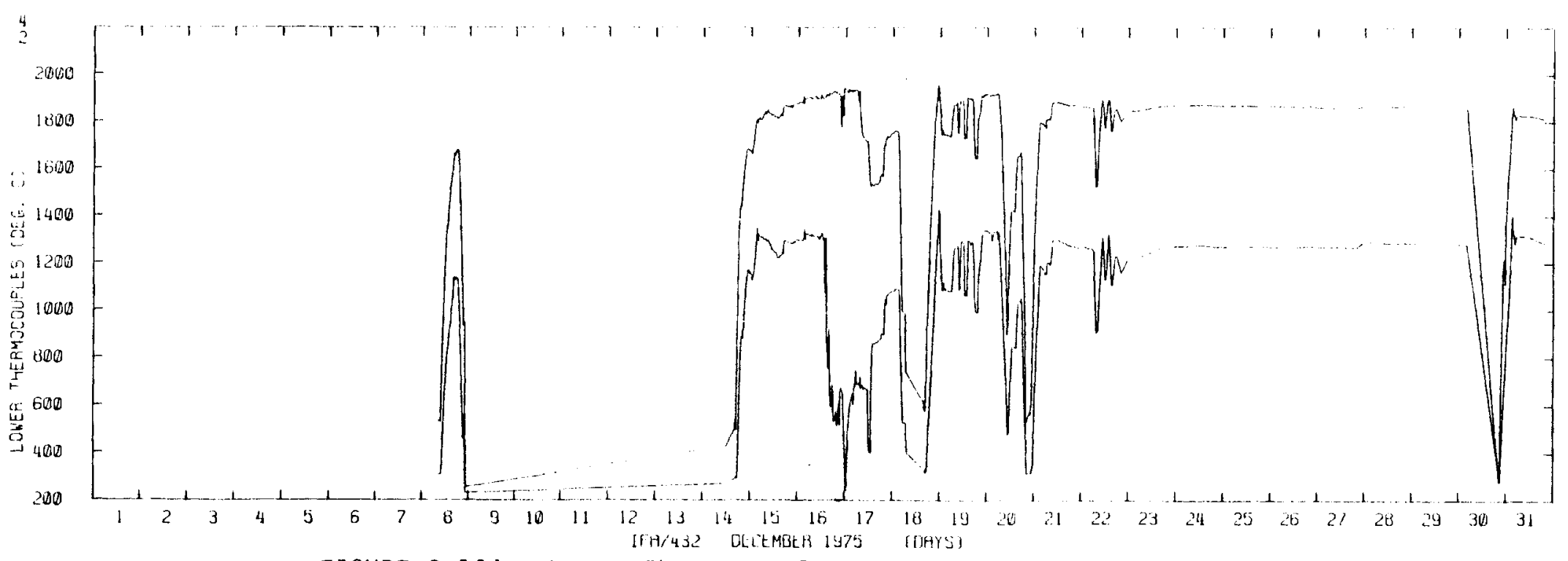

FIGURE 3.124. Lower Thermocouple Readings for Rods 2,4 of IFA 432 - December 1975 


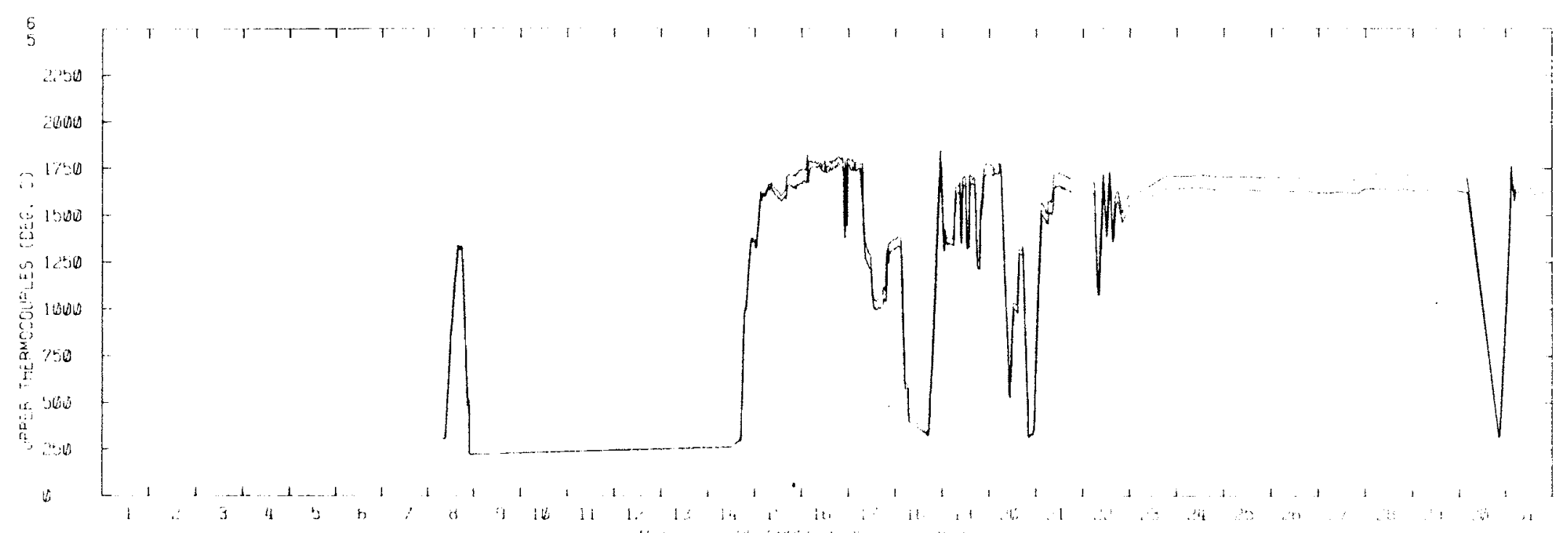

FIGURE 3.125. Upper Thermocouple Readings for Rods 5,6 of IFA 432 - December 1975

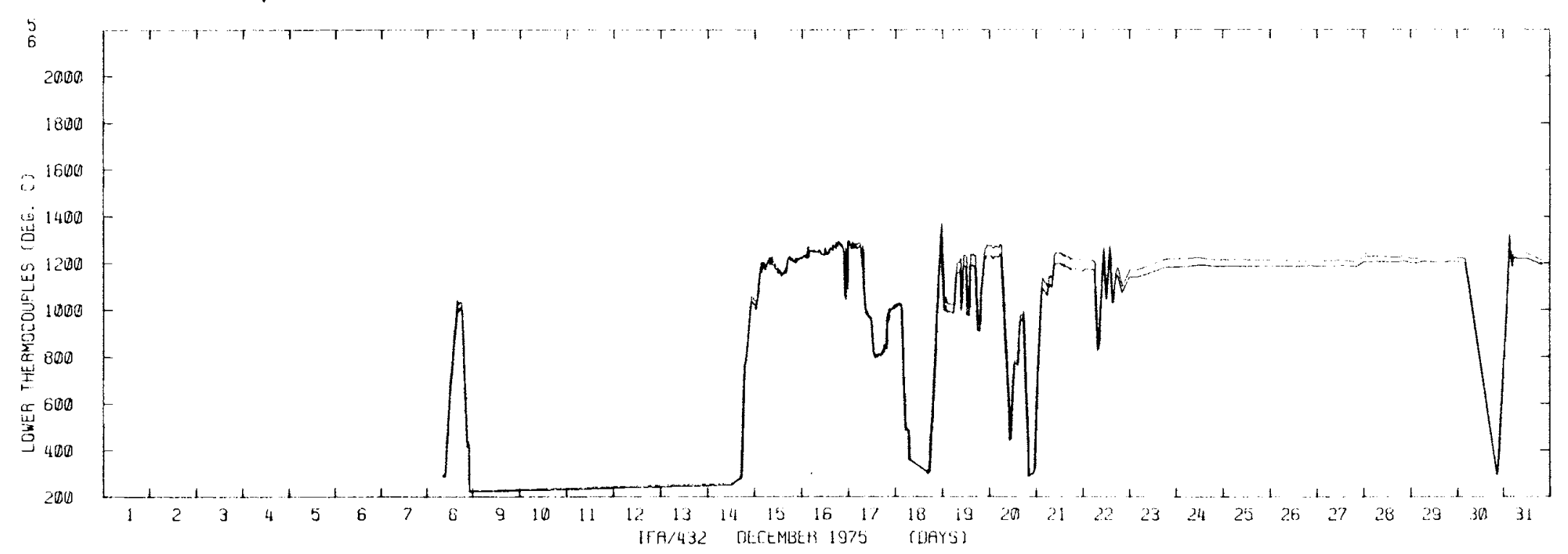

FIGURE 3.126. Lower Thermocouple Readings for Rods 5,6 of IFA 432 - December 1975 


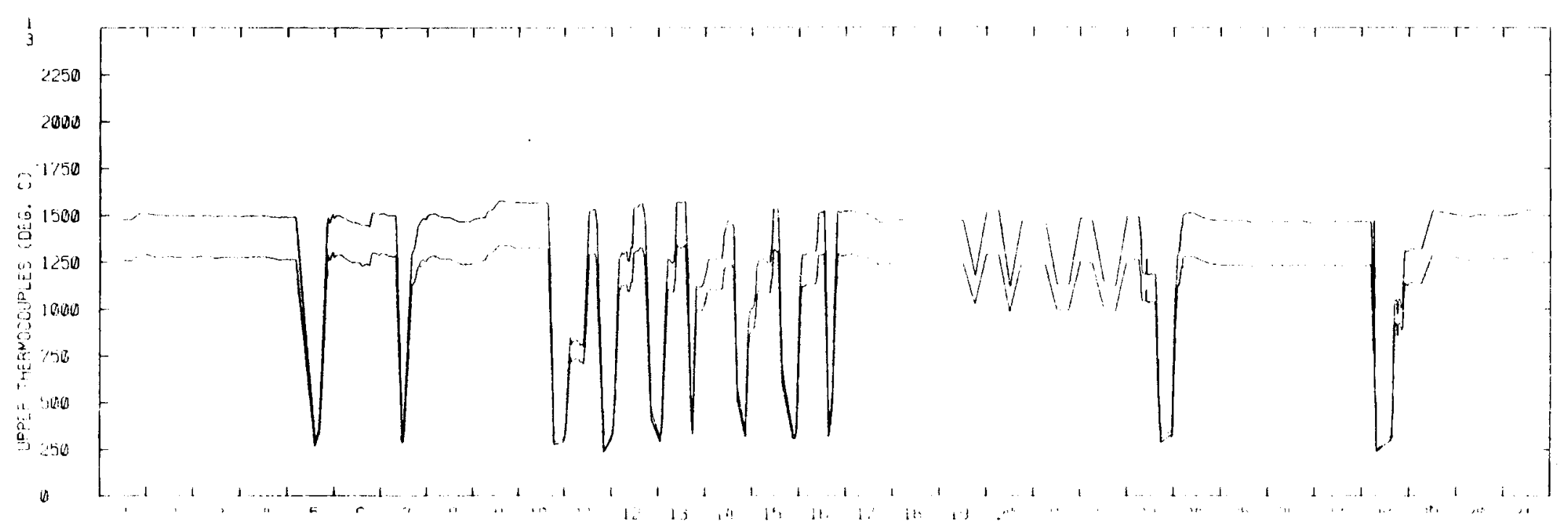

ஸ́

FIGURE 3.127. Upper Thermocouple Readings for Rods 1,3 of IFA 432 - January 1976

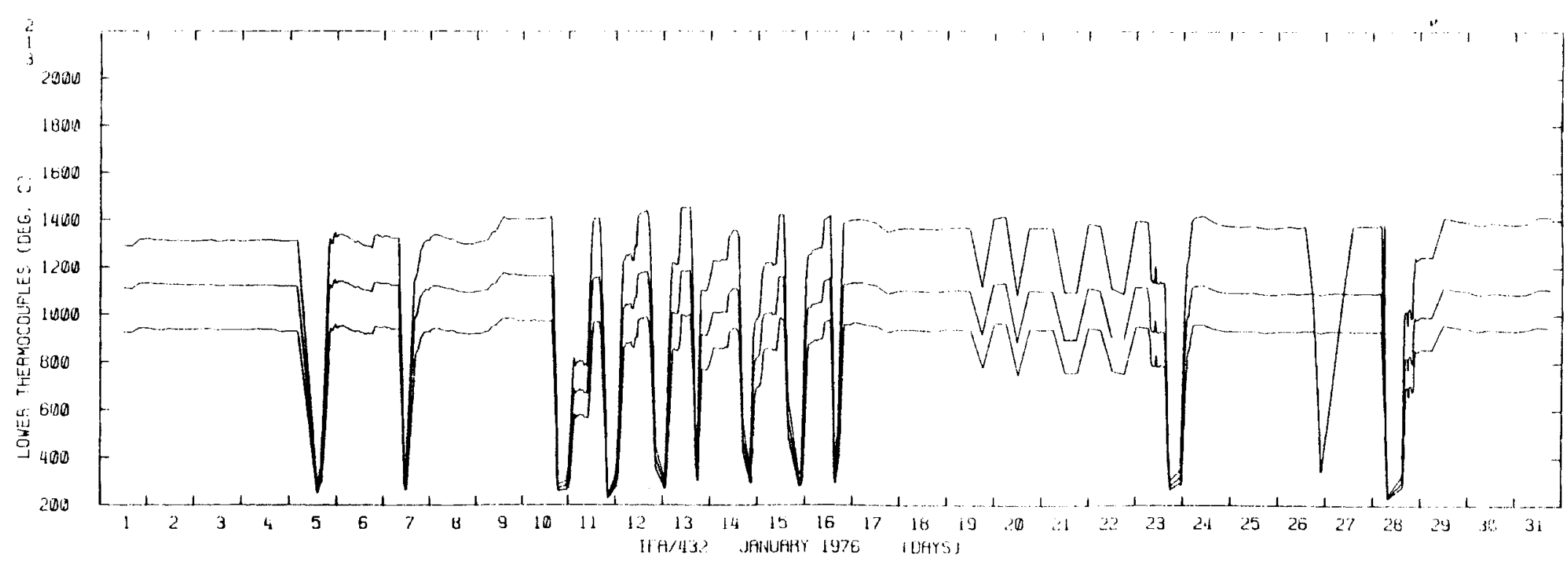

FIGURE 3.128. Lower Thermocouple Readings for Rods 1,2,3 of IFA 432 - January 1976 


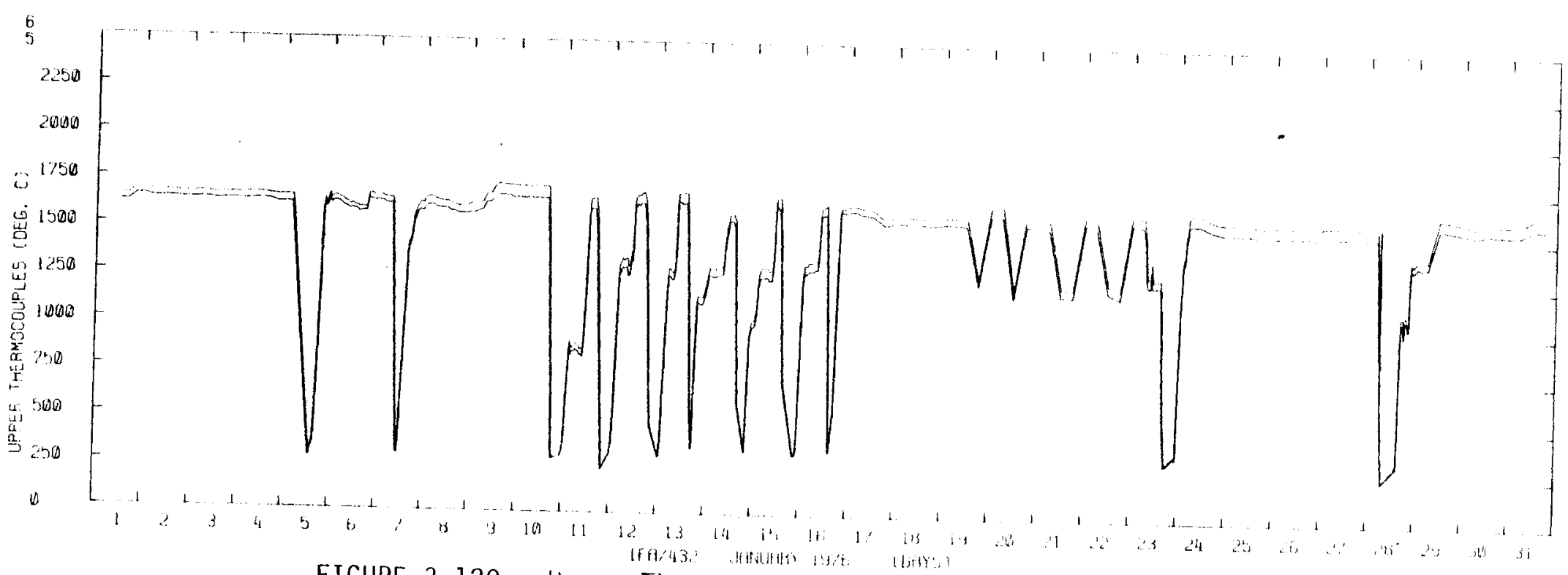

$\stackrel{w}{1}$

FIGURE 3.129. Upper Thermocouple Readings for Rods 5,6

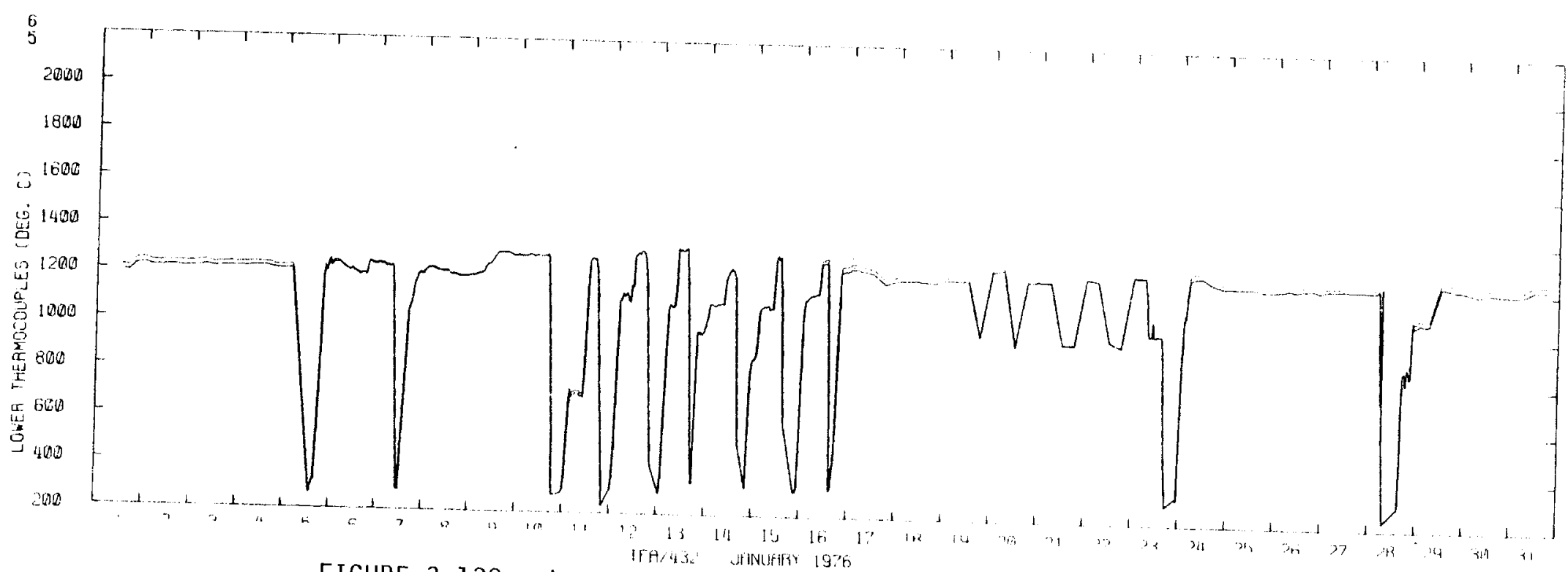

FIGURE 3.130. Lower Thermocouple Readings for Rods 5,6 of IFA 432 - January 1976 


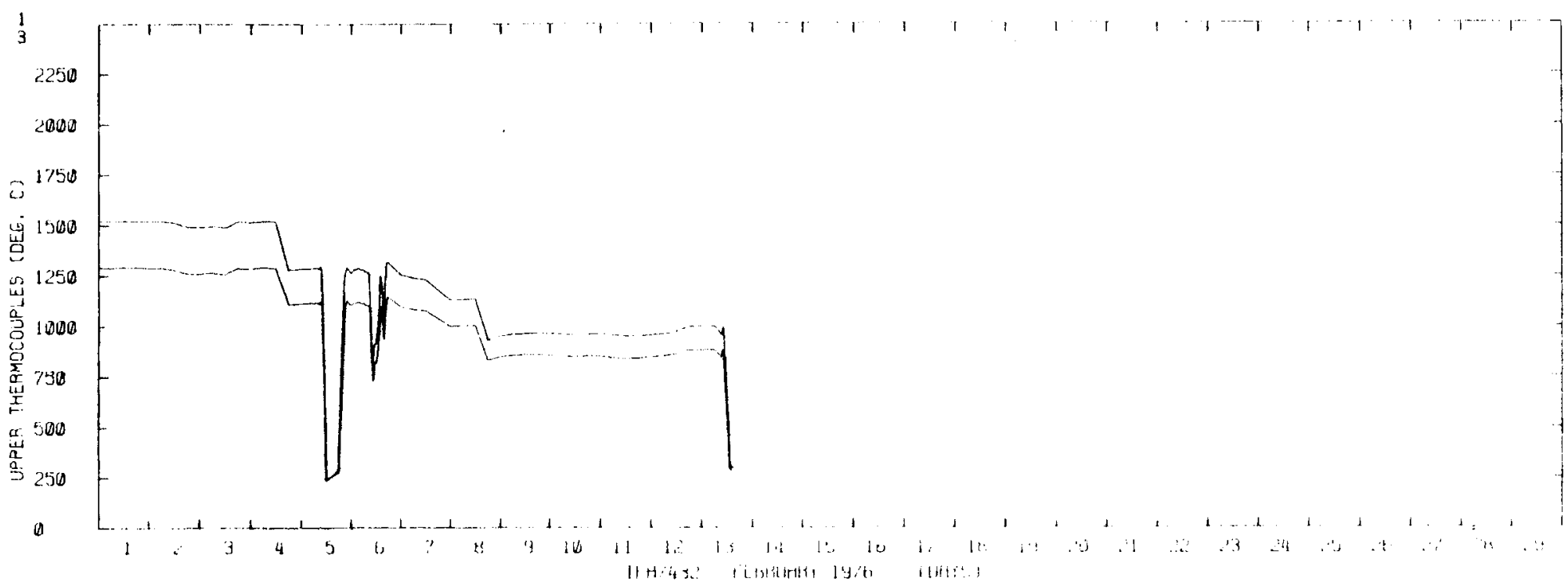

FIGURE 3.131. Upper Thermocouple Readings for Rods 1,3 of IFA 432 - February 1976

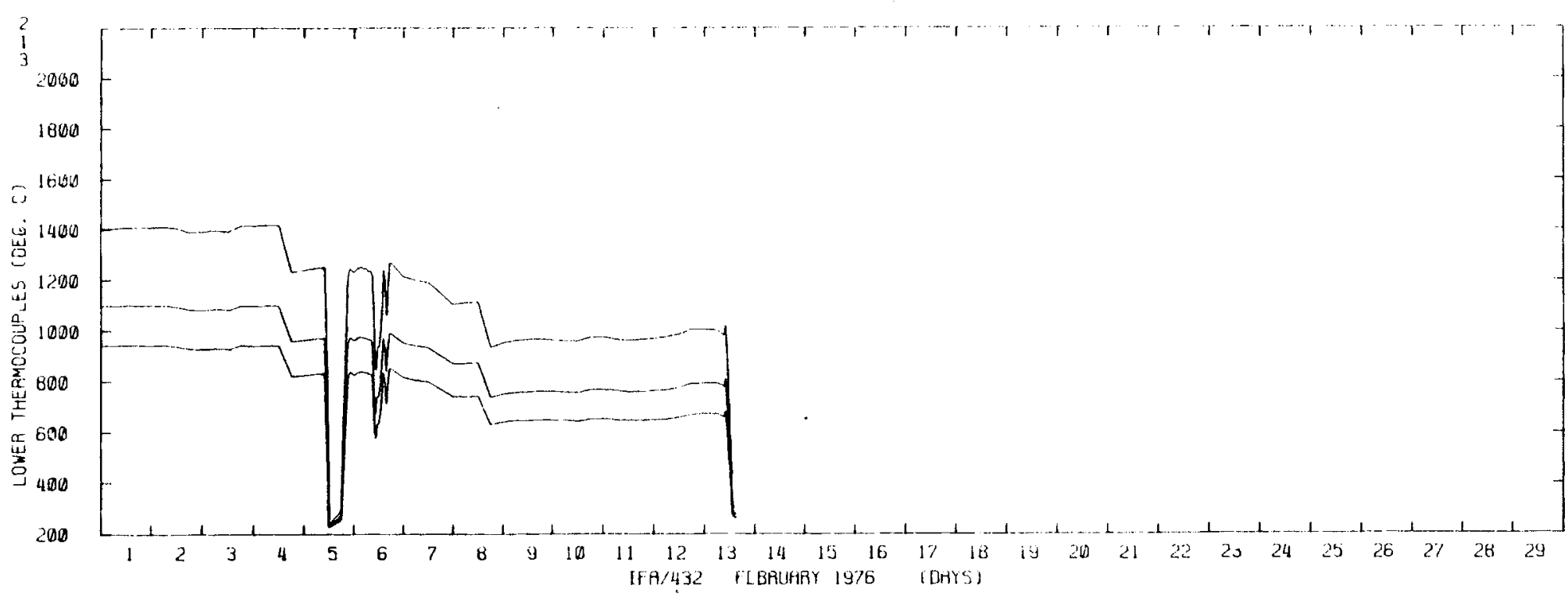

FIGURE 3.132. Lower Thermocouple Readings for Rods 1, 2, 3 of IFA 432 - February 1976 


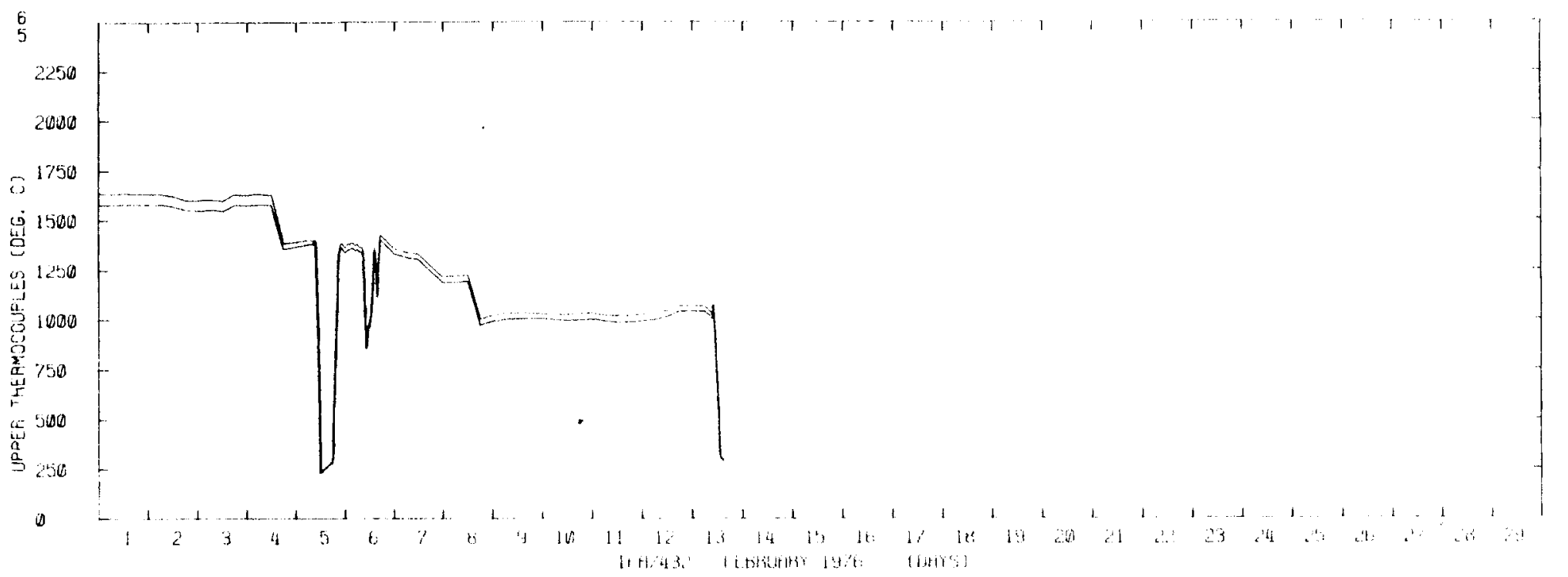

$\omega$
1
$\sigma$

FIGURE 3.133. Upper Thermocouple Readings for Rods 5,6 of IFA 432 - February 1976

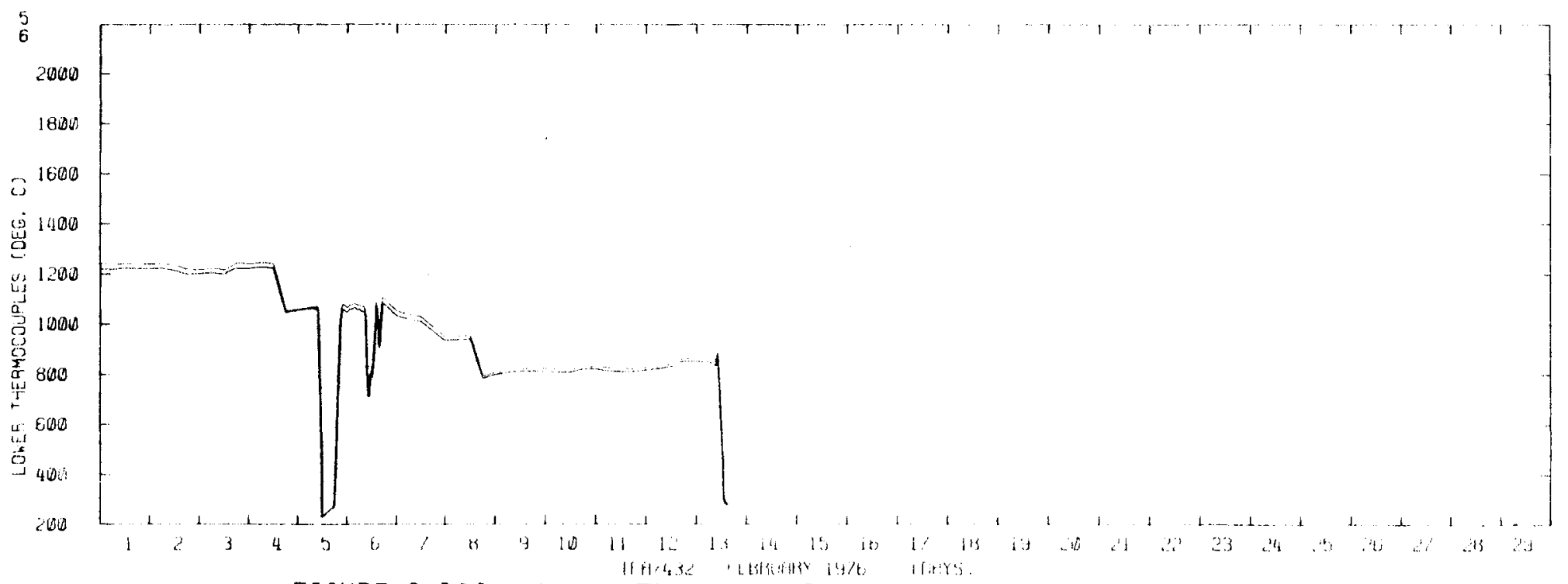

FIGURE 3.134. Lower Thermocouple Readings for Rods 5,6 of IFA 432 - February 1976 


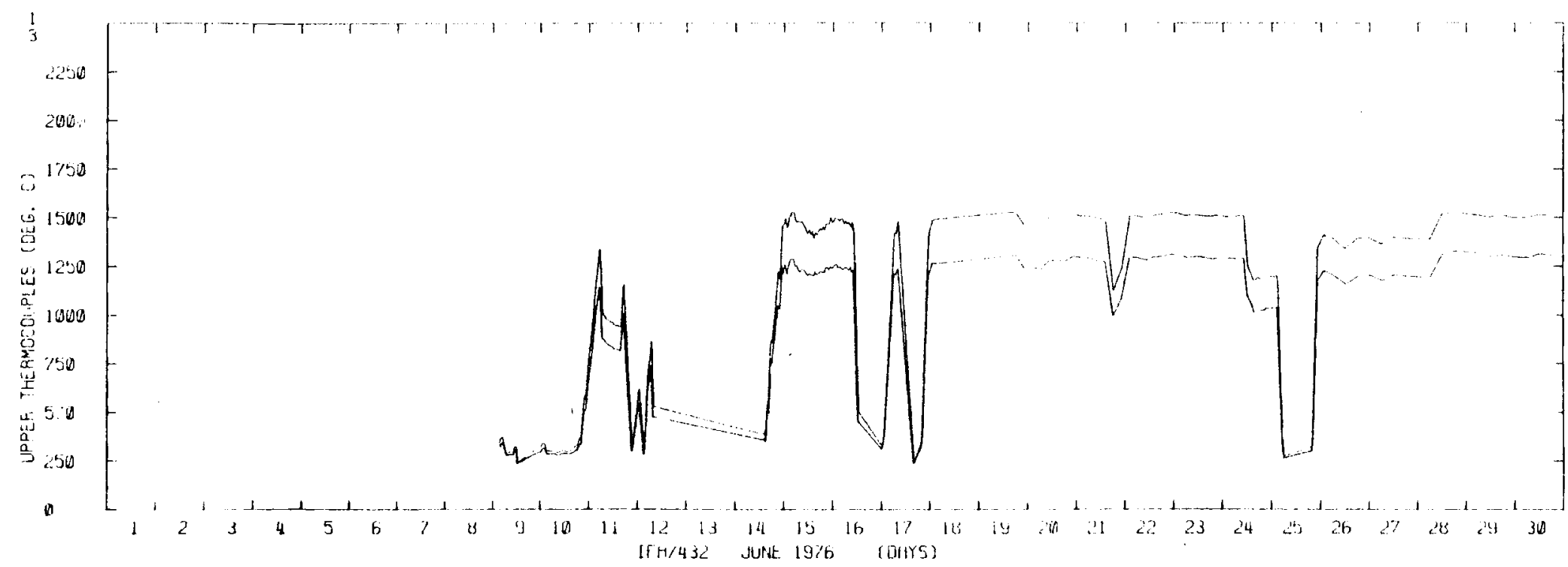

FIGURE 3.135. Upper Thermocouple Readings for Rods 1,3 of IFA 432 - June 1976

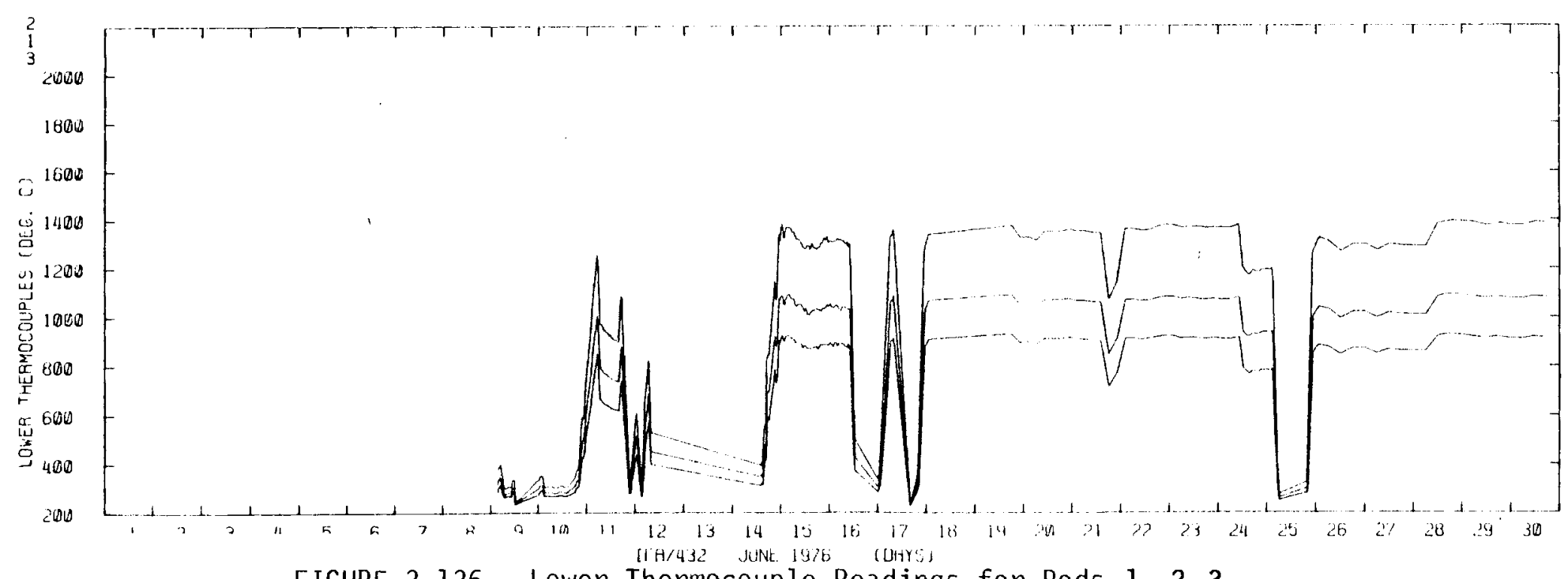

FIGURE 3.136. Lower Thermocouple Readings for Rods 1, 2, 3 of IFA 432 - June 1976 


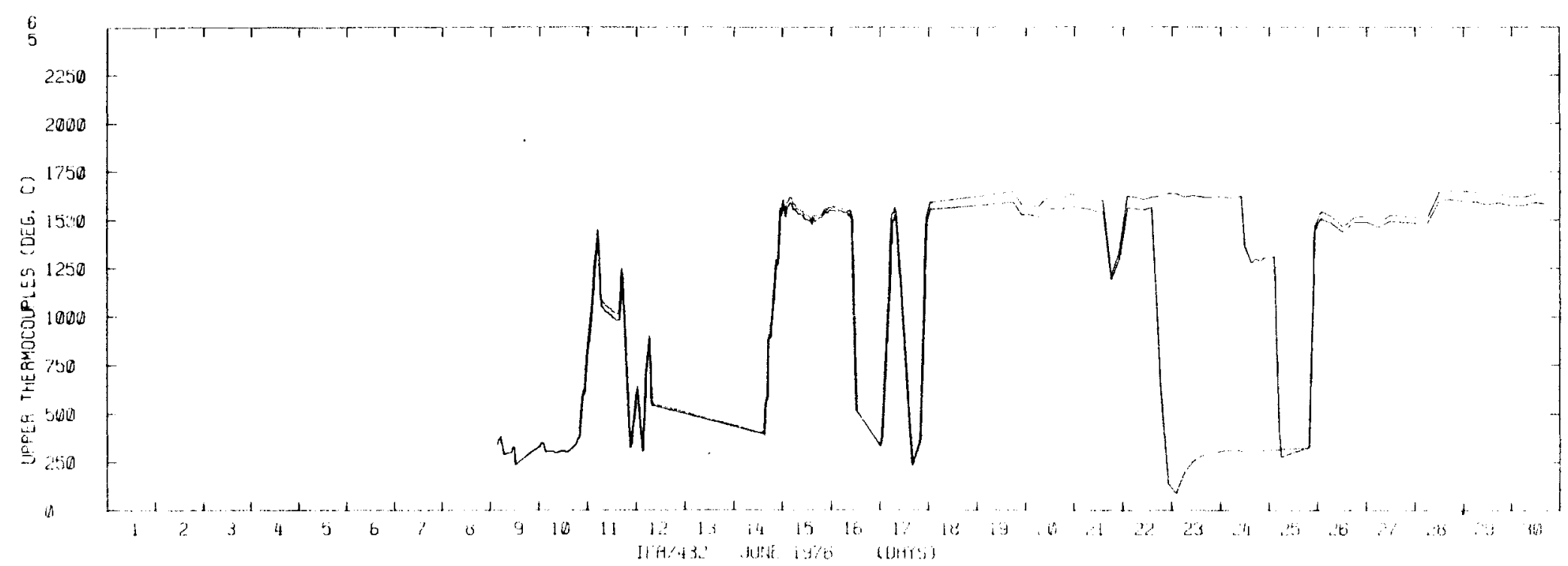

$\stackrel{\omega}{\perp}$

FIGURE 3.137. Upper Thermocouple Readings for Rods 5,6 of IFA 432 - June 1976

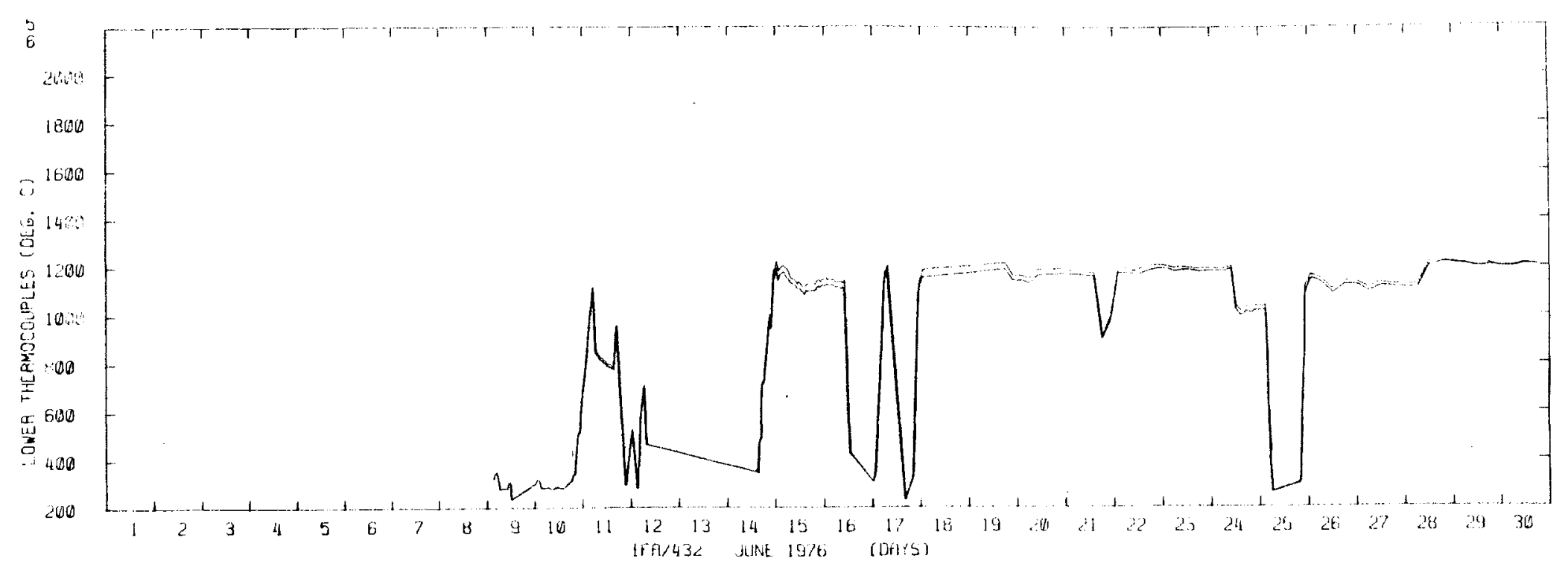

FIGURE 3.138. Lower Thermocouple Readings for Rods 5,6 of IFA 432 - June 1976 


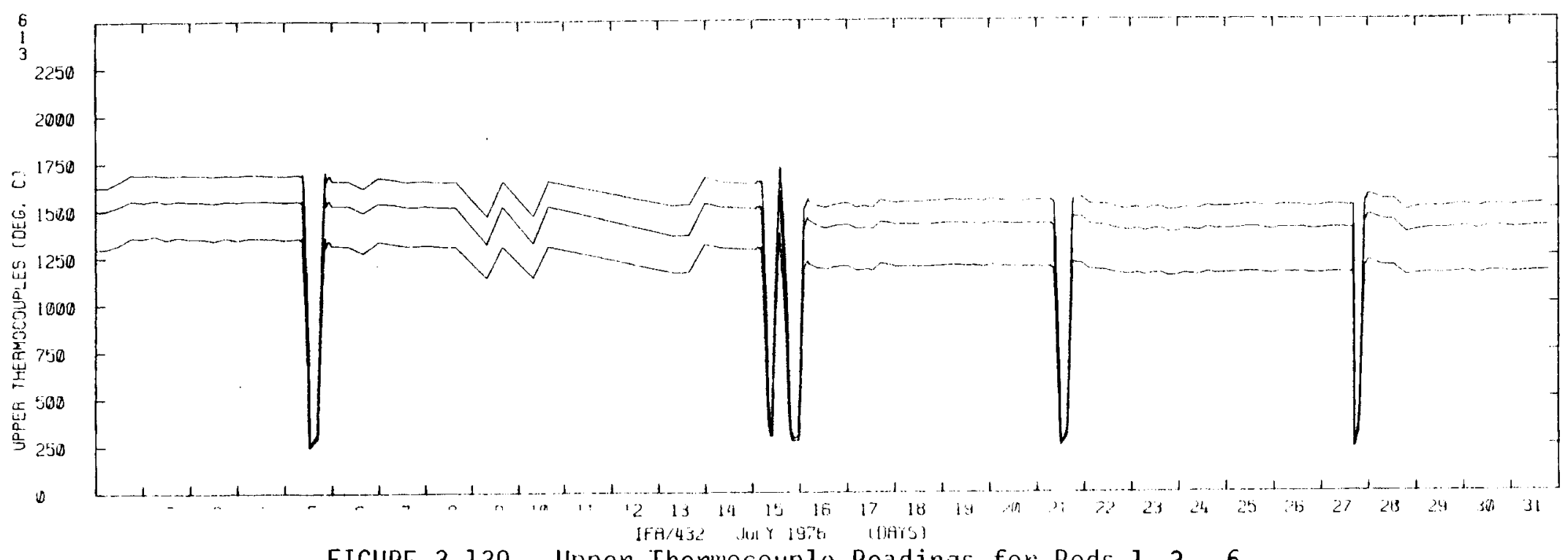

FIGURE 3.139. Upper Thermocouple Readings for Rods 1, 3,6 of IFA 432 - July 1976

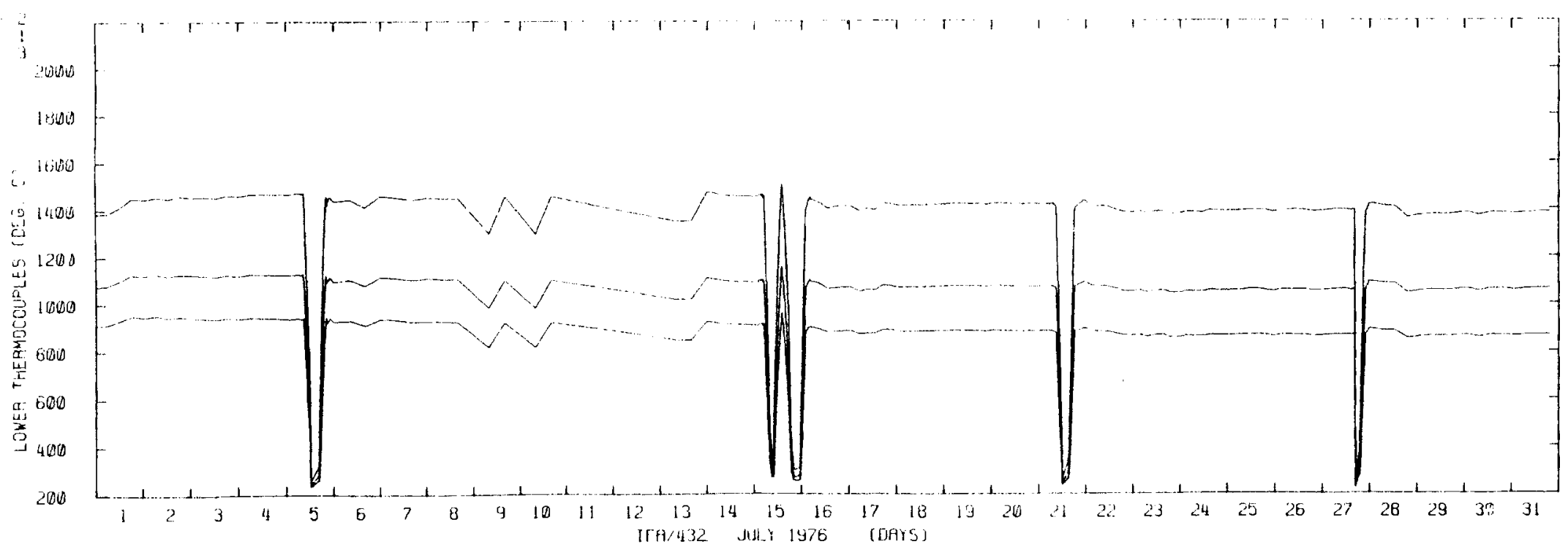

FIGURE 3.140. Lower Thermocouple Readings for Rods $1,2,3$ of IFA 432 - JuTy 1976 


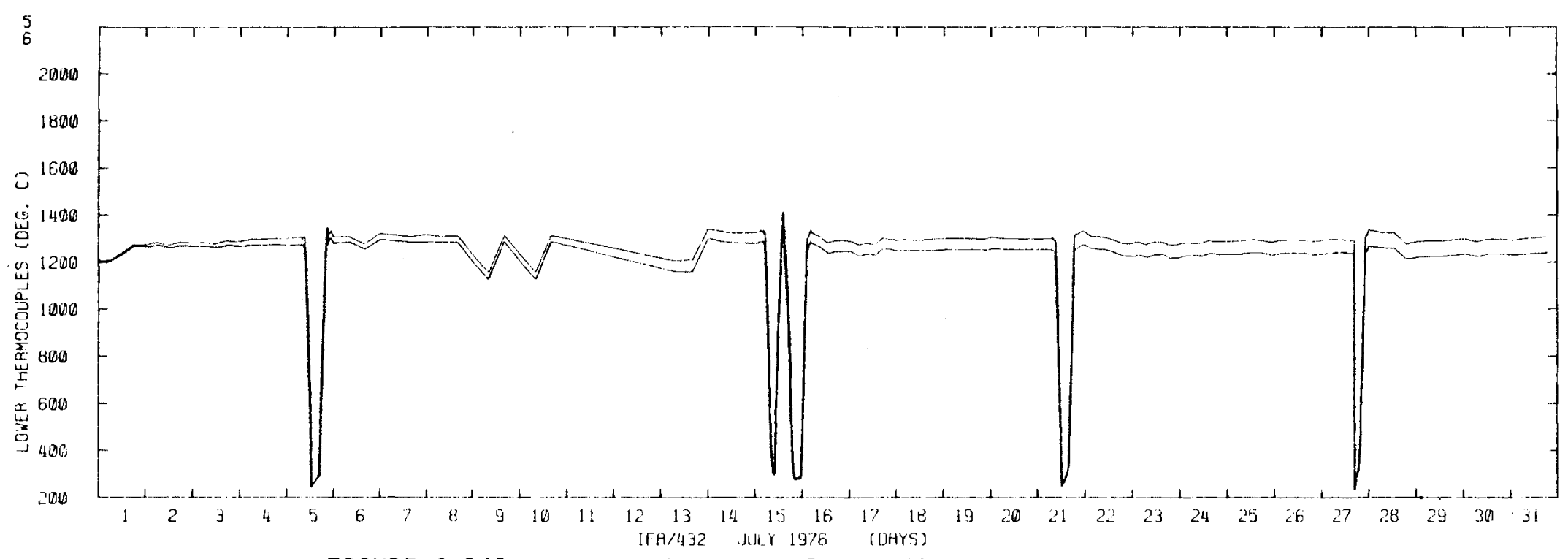

$\stackrel{\omega}{\omega}_{\omega}^{\omega}$

FIGURE 3.141. Lower Thermocouple Readings for Rods 5,6 of IFA 432 - July 1976

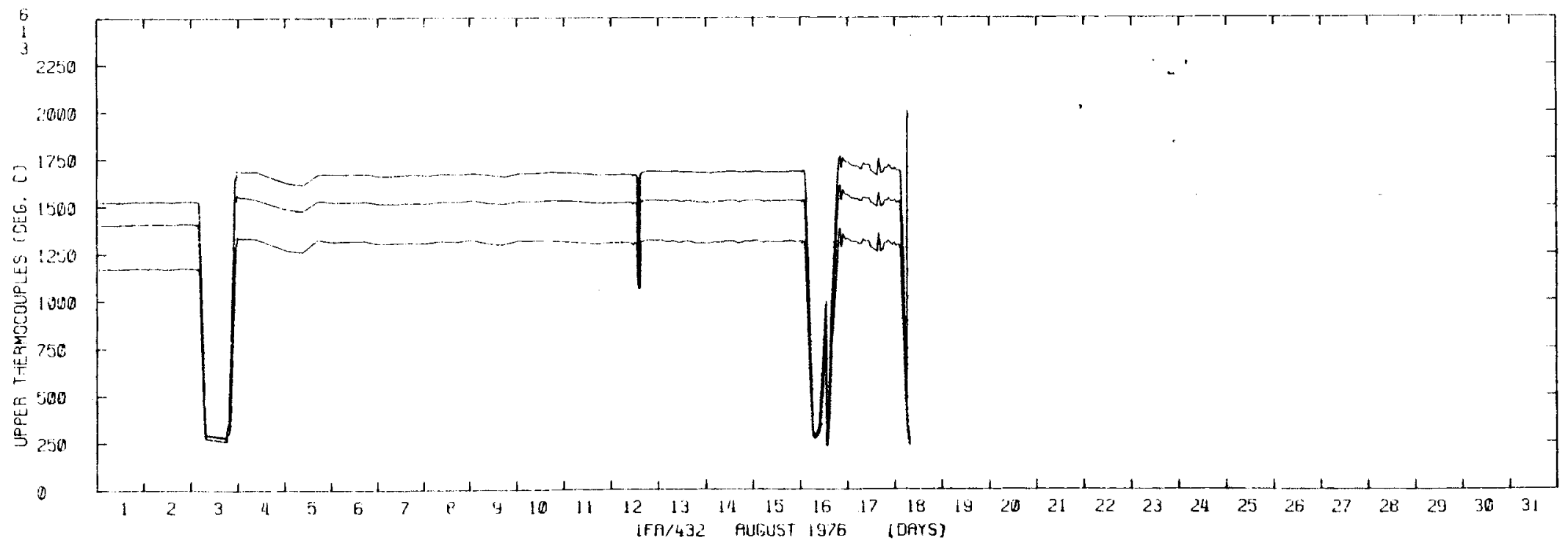

FIGURE 3.142. Upper Thermocouple Readings for Rods 1,3,6 of IFA 432 - August 1976 


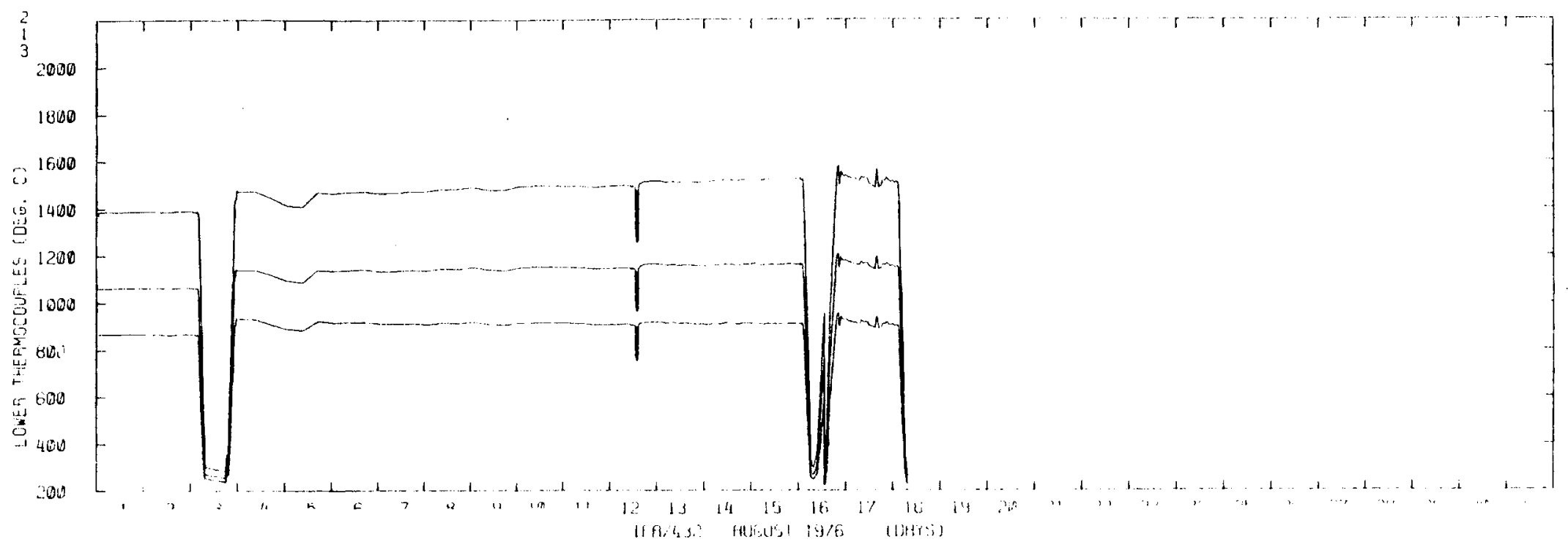

FIGURE 3.143. Lower Thermocouple Readings for Rods 1, 2, 3 of IFA 432 - August 1976

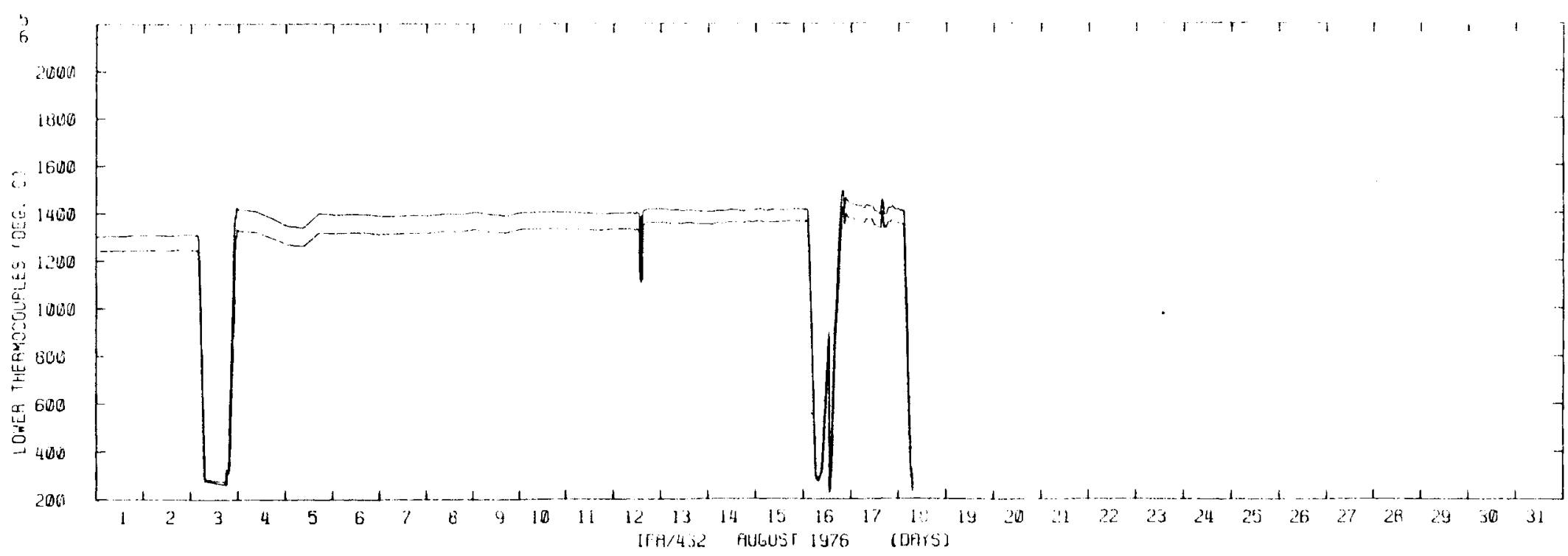

FIGURE 3.144. Lower Thermocouple Readings for Rods 5,6 of IFA 432 - August 1976 


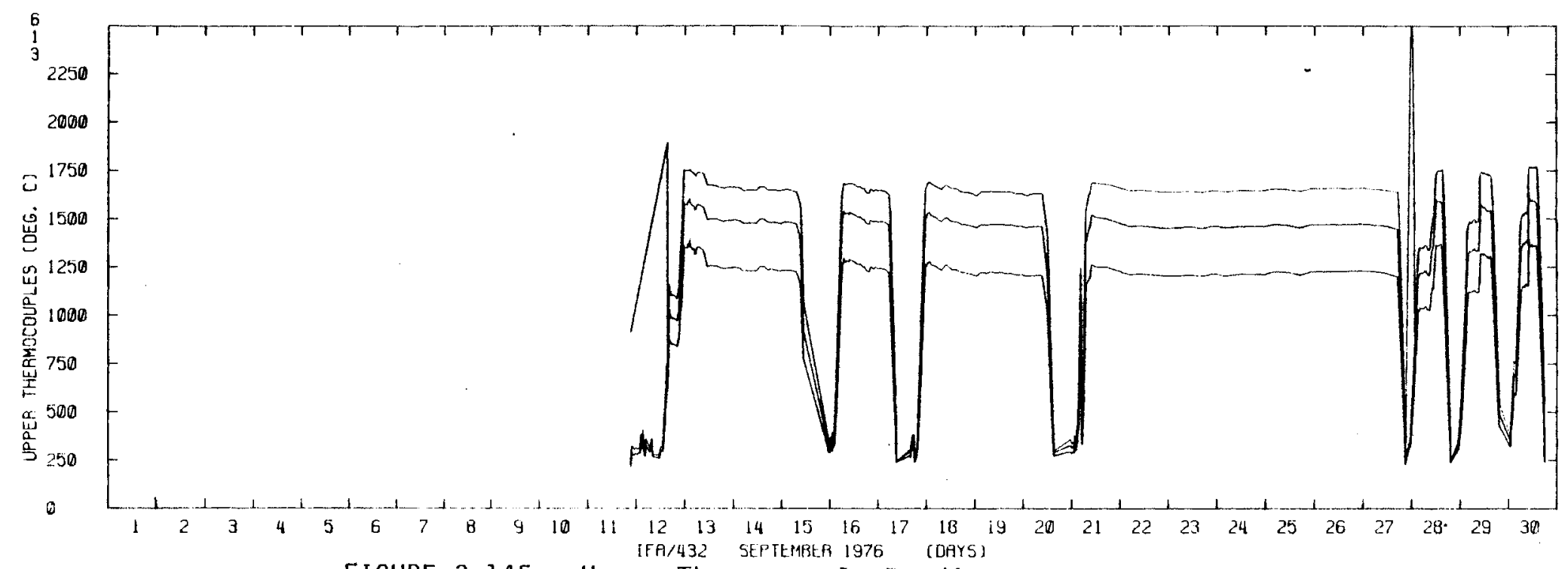

$\stackrel{\omega}{\dot{1}}$

FIGURE 3.145. Upper Thermocouple Readings for Rods 1, 3, 6 of IFA 432 - September 1976

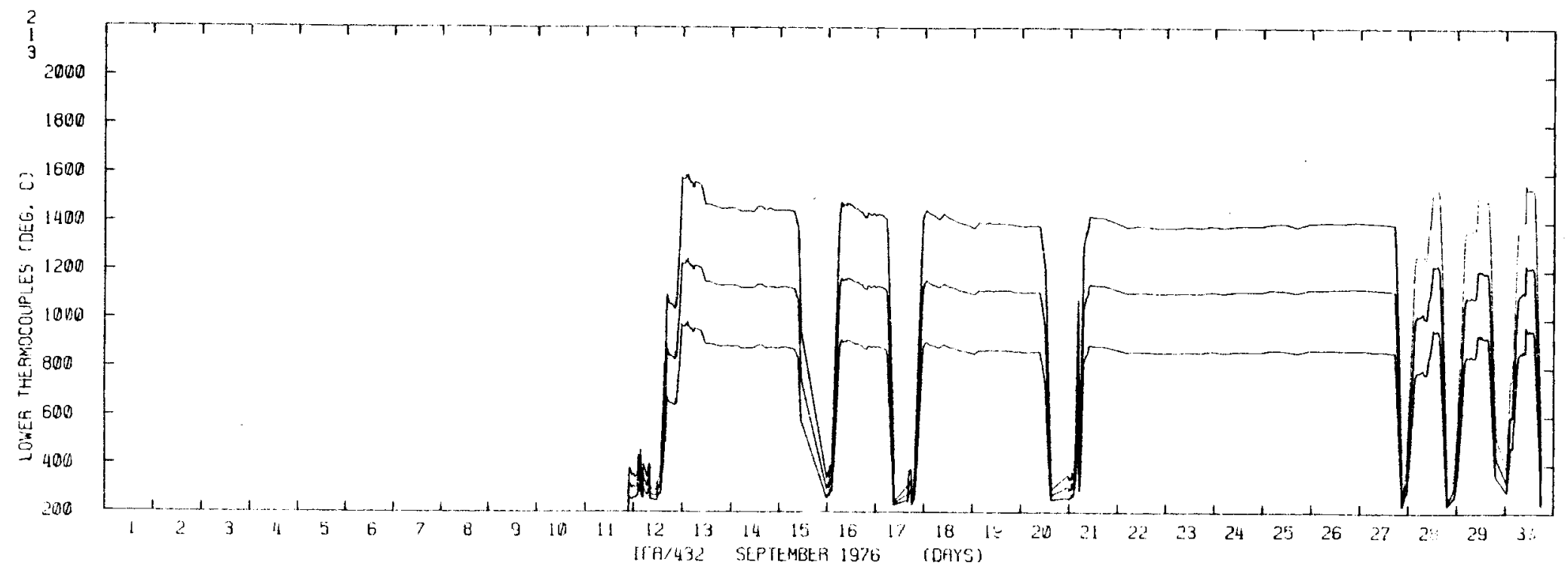

FIGURE 3.146. L.ower Thermocouple Readings for Rods $1,2,3$ of IFA 432 - September 1976 


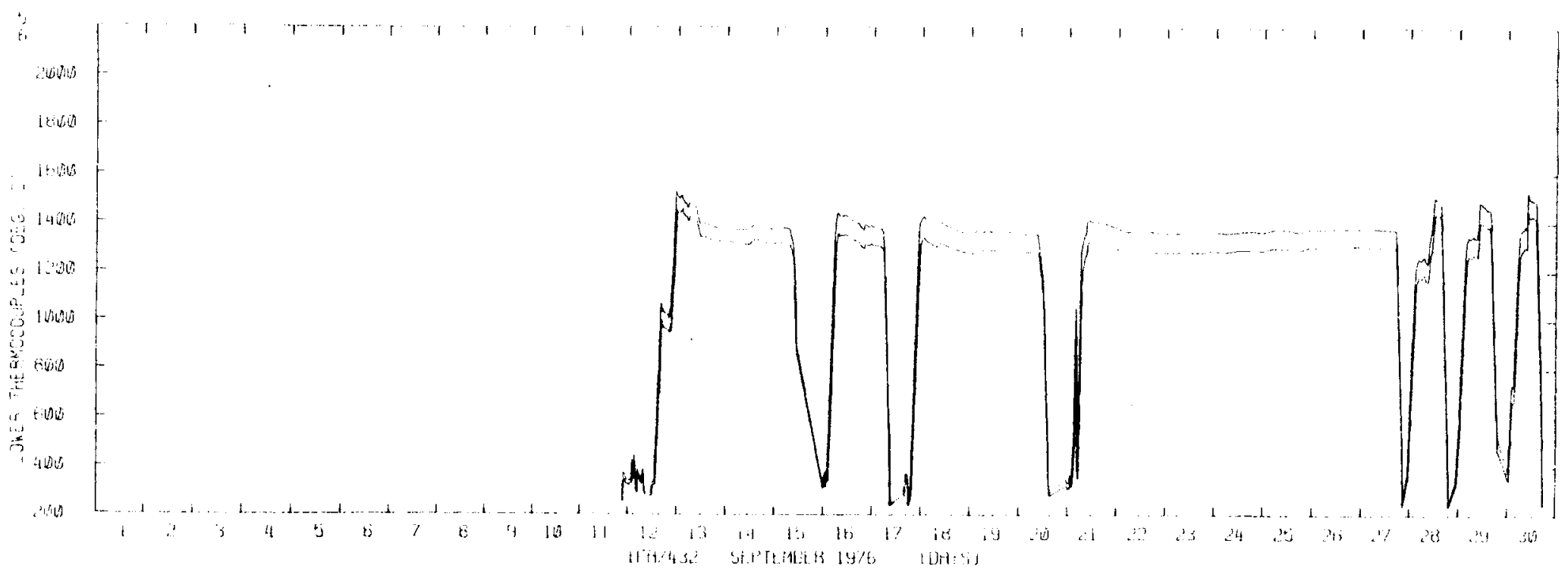

$\stackrel{w}{1}$

FIGURE 3.147. Lower Thermocouple Readings for Rods 5,6 of IFA 432 - September 1976

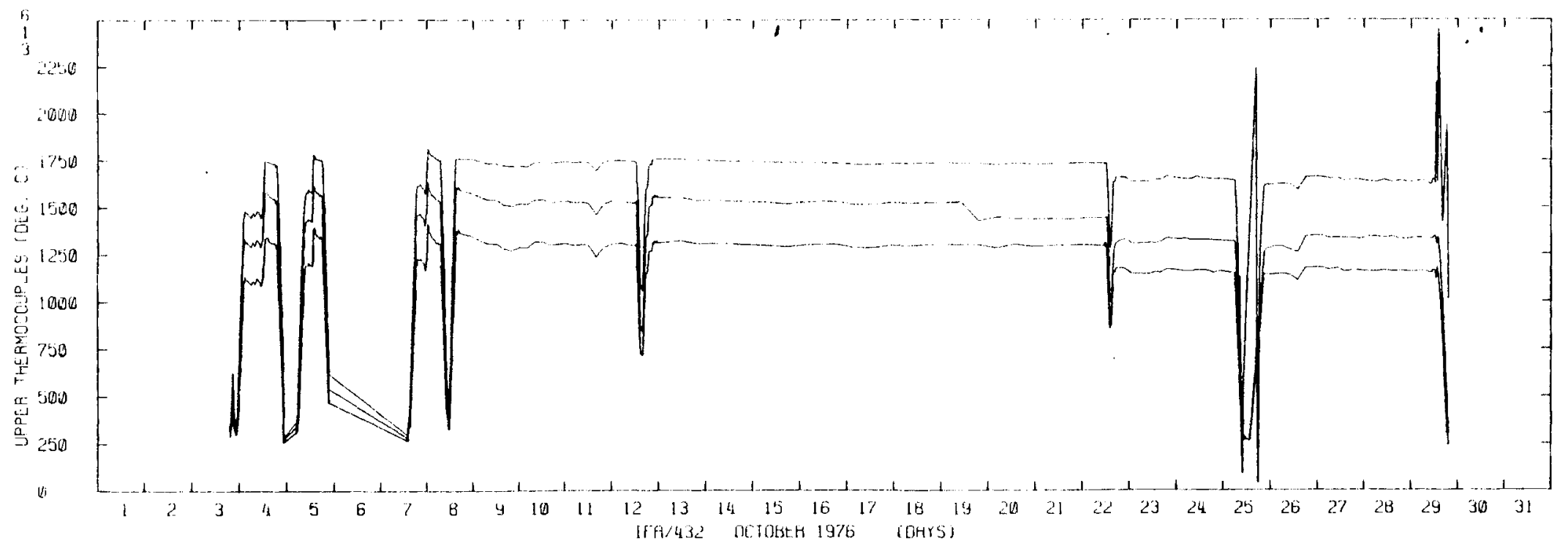

FIGURE 3.148. Upper Thermocouple Readings for Rods 1, 3, 6 of IFA 432 - October 1976 


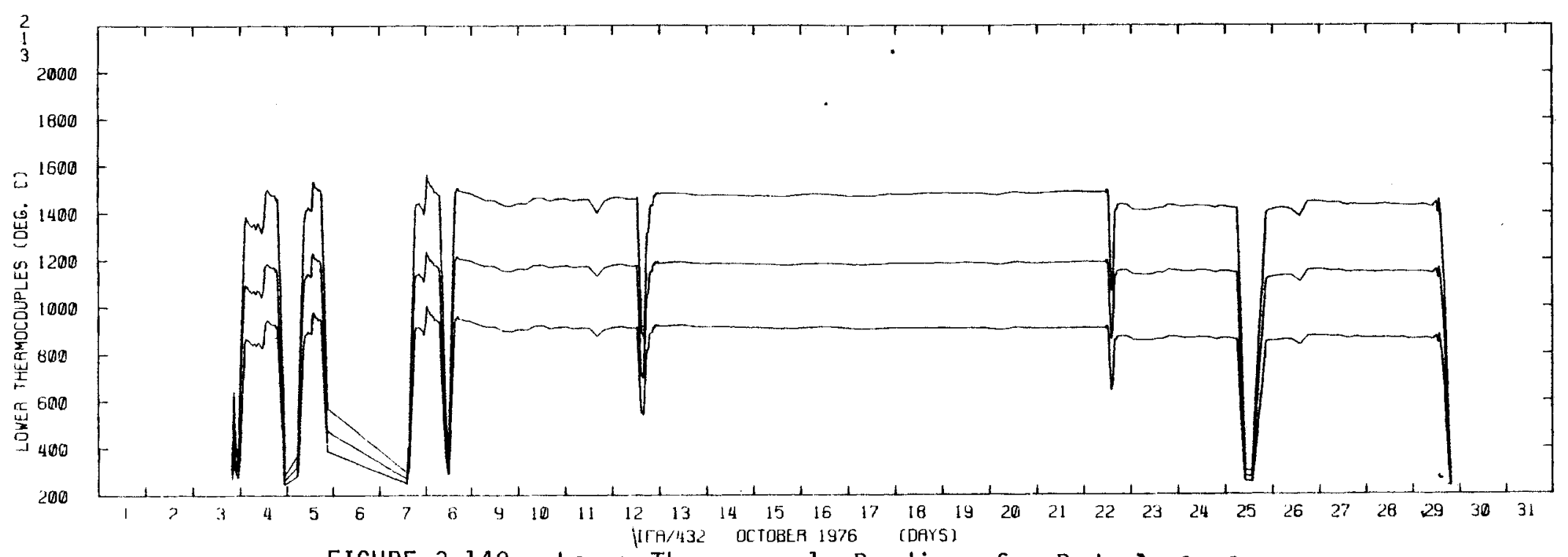

$\stackrel{w}{y}$

FIGURE 3.149. Lower Thermocouple Readings for Rods $1,2,3$

of IFA 432 - October 1976

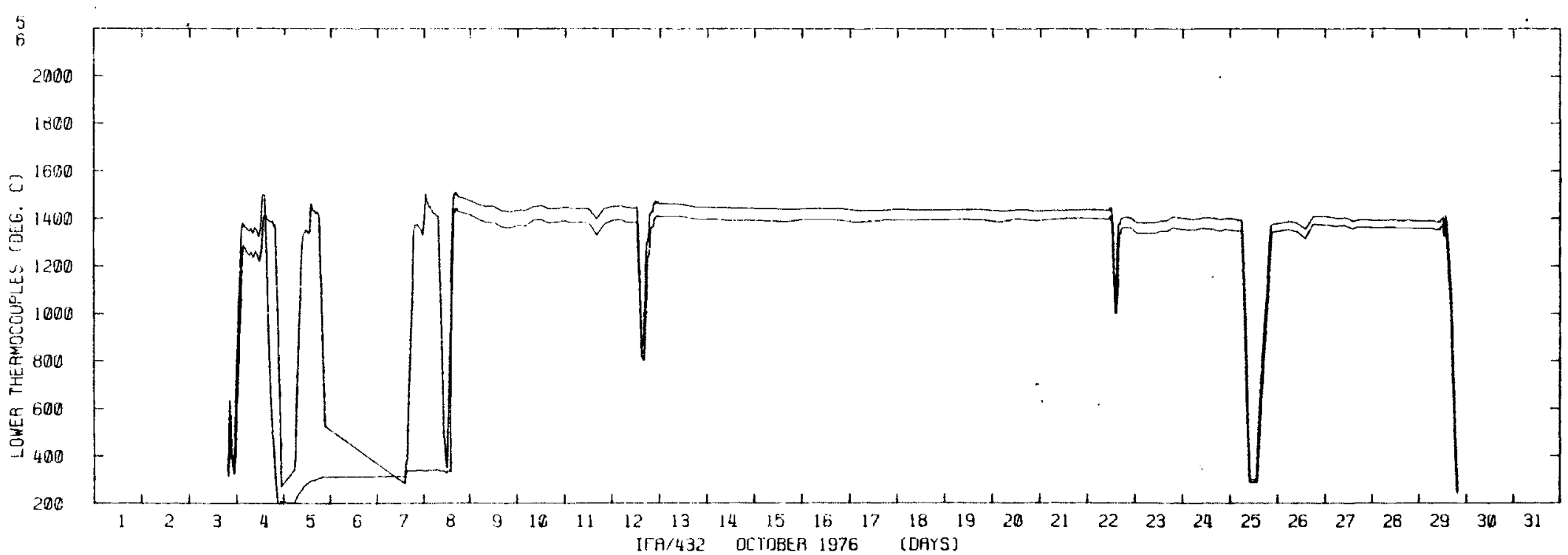

FIGURE 3.150. Lower Thermocouple Readings for Rods 5,6 of IFA 432 - October 1976 


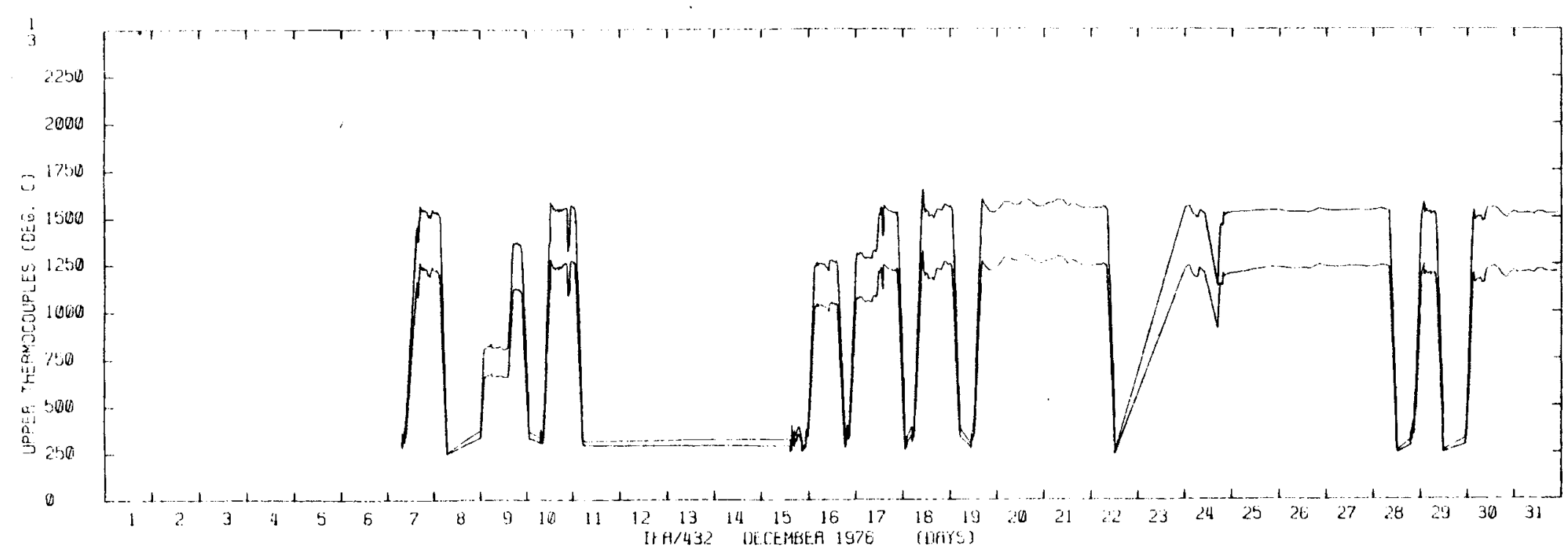

FIGURE 3.151. Upper Thermocouple Readings for Rods 1,3 of IFA 432 - December 1976

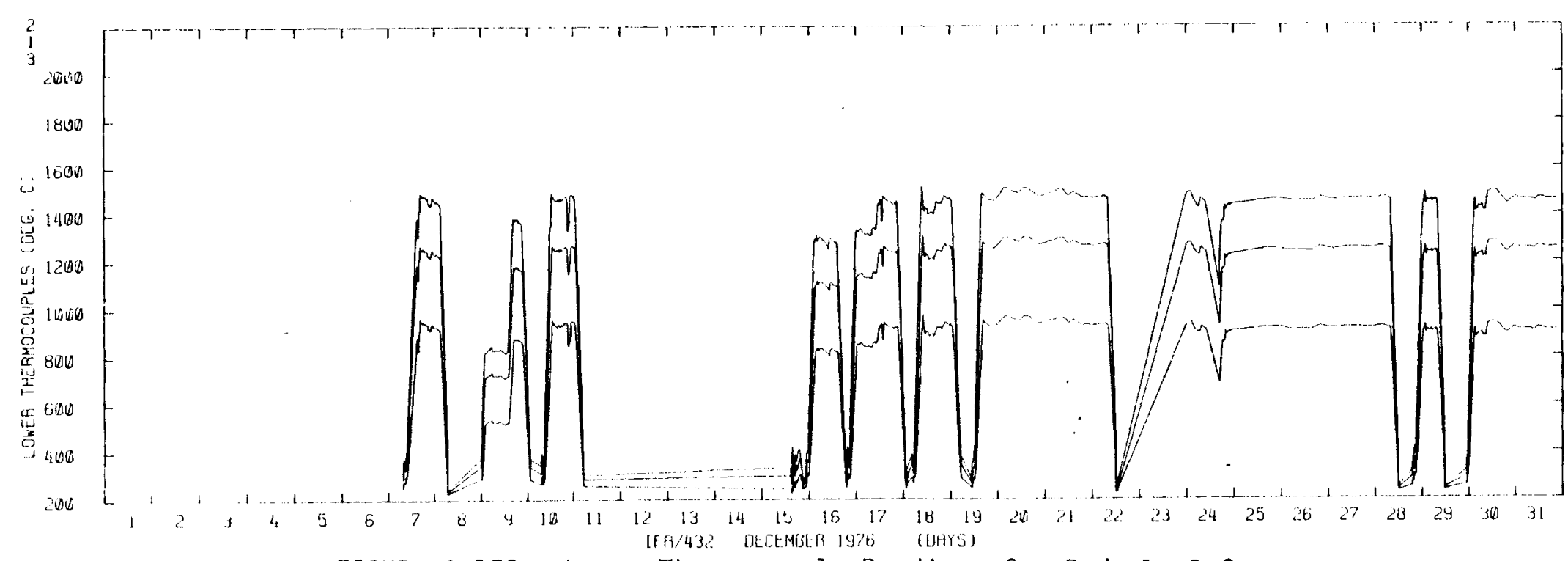

FIGURE 3.152. Lower Thermocouple Readings for Rods 1, 2,3 of IFA 432 - December 1976 


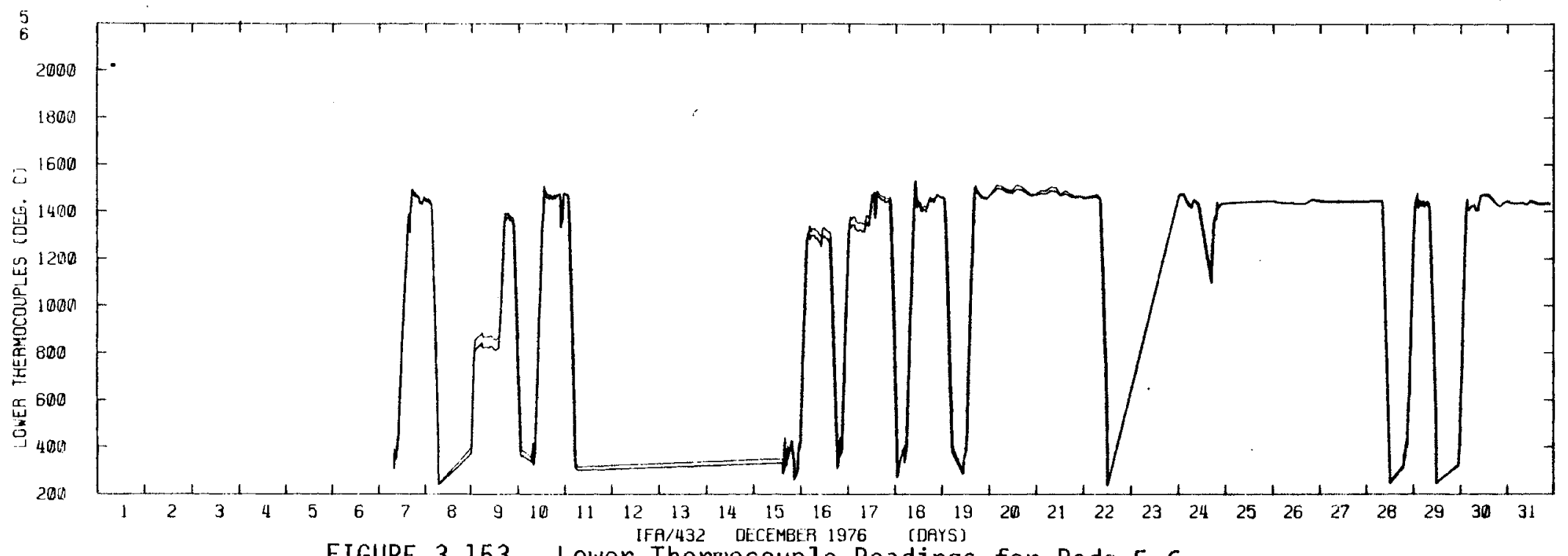

$\frac{\omega}{1}$

FIGURE 3.153. Lower Thermocouple Readings for Rods 5,6 of IFA 432 - December 1976

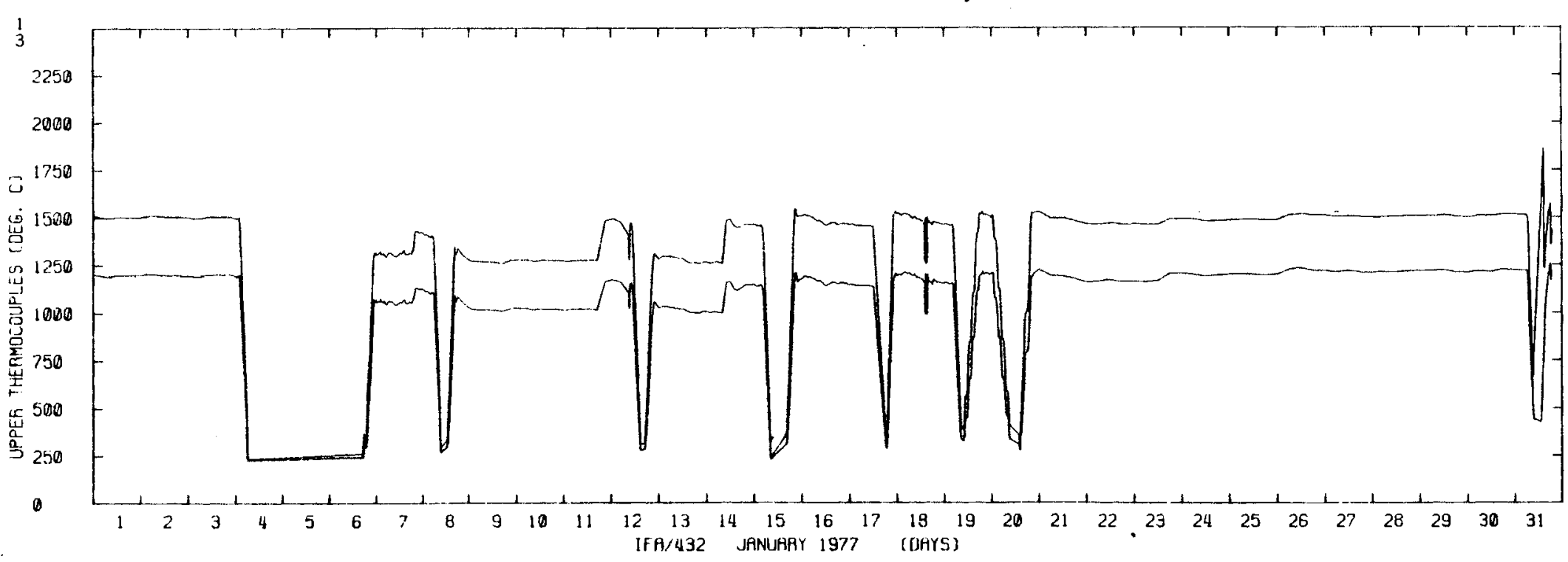

FIGURE 3.154. Upper Thermocouple Readings for Rods 1,3 of IFA 432 - January 1977 


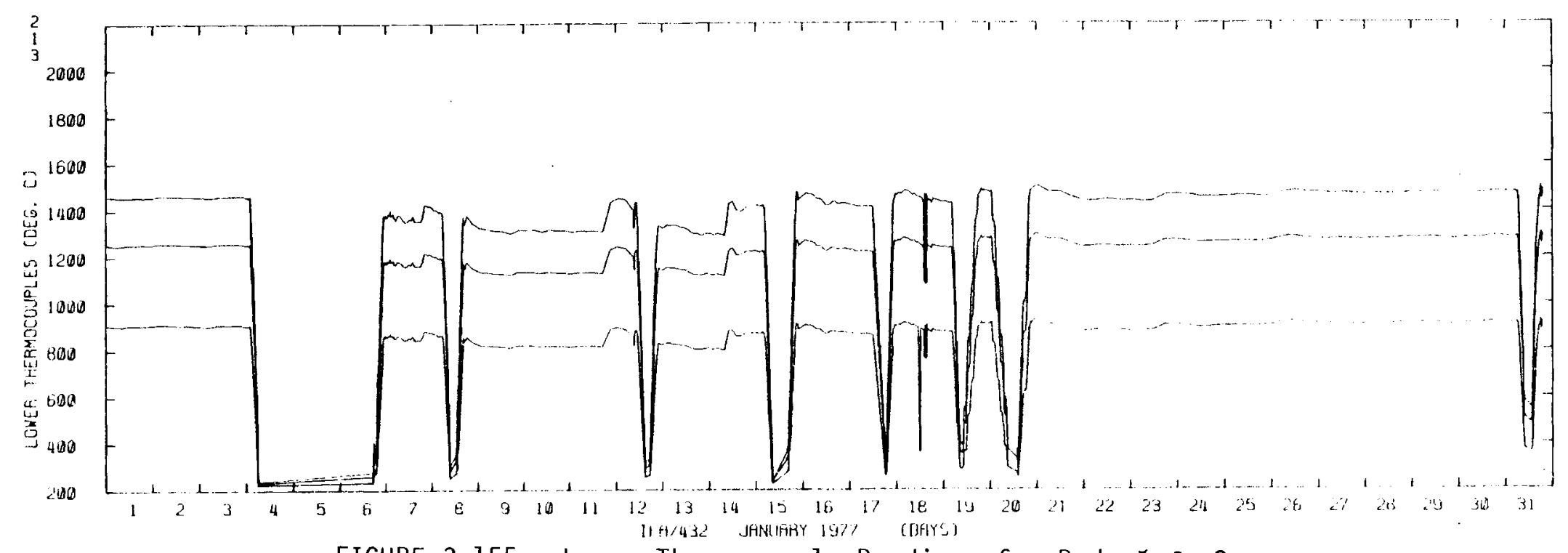

$\omega$
1
0

FIGURE 3.155. Lower Thermocouple Readings for Rods 1,2,3 of IFA 432 - January 1977

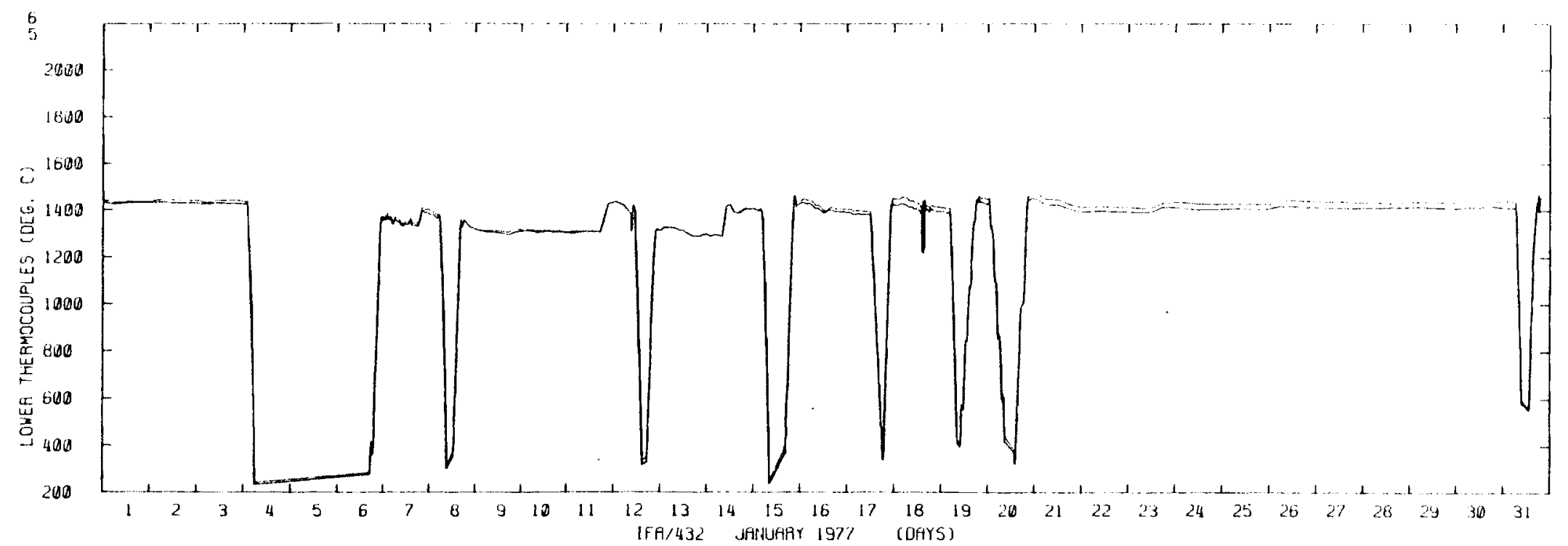

FIGURE 3.156. Lower Thermocouple Readings for Rods 5,6 of IFA 432 - January 1977 


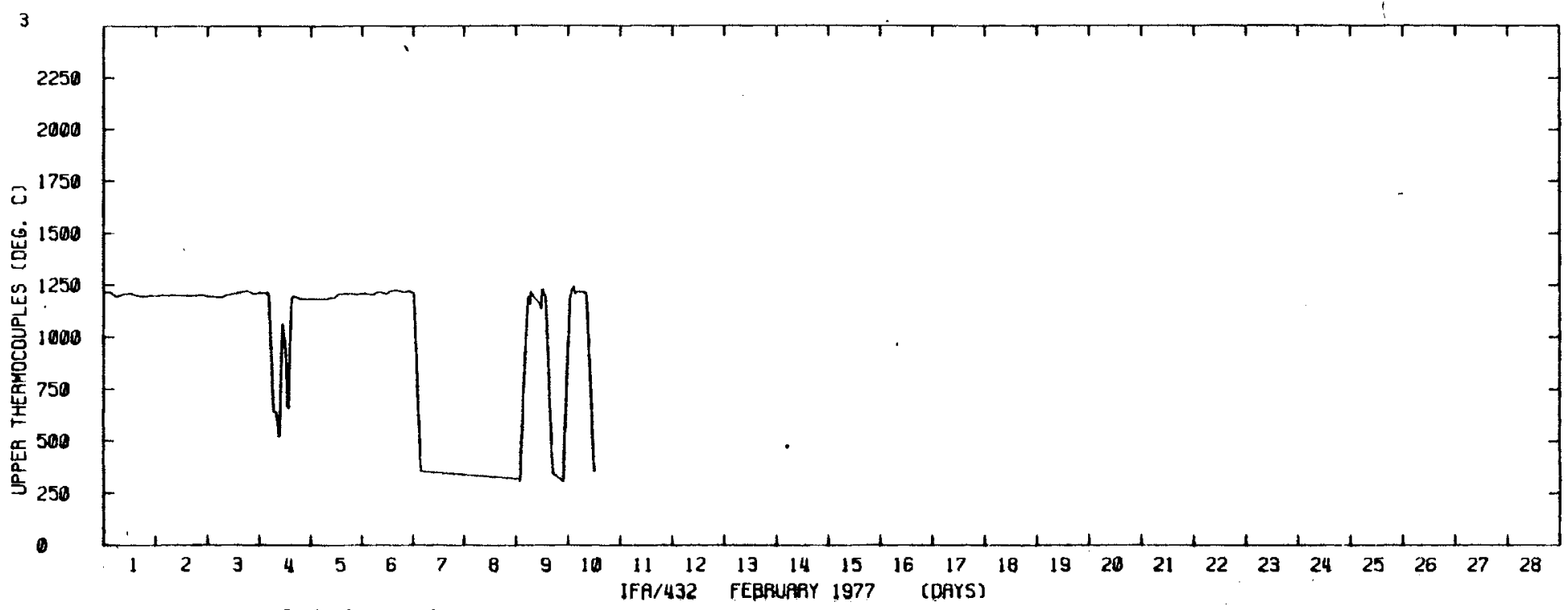

$\omega$
1
$\infty$

FIGURE 3.157. Upper Thermocouple Readings for Rod 3 of IFA 432 - February 1977

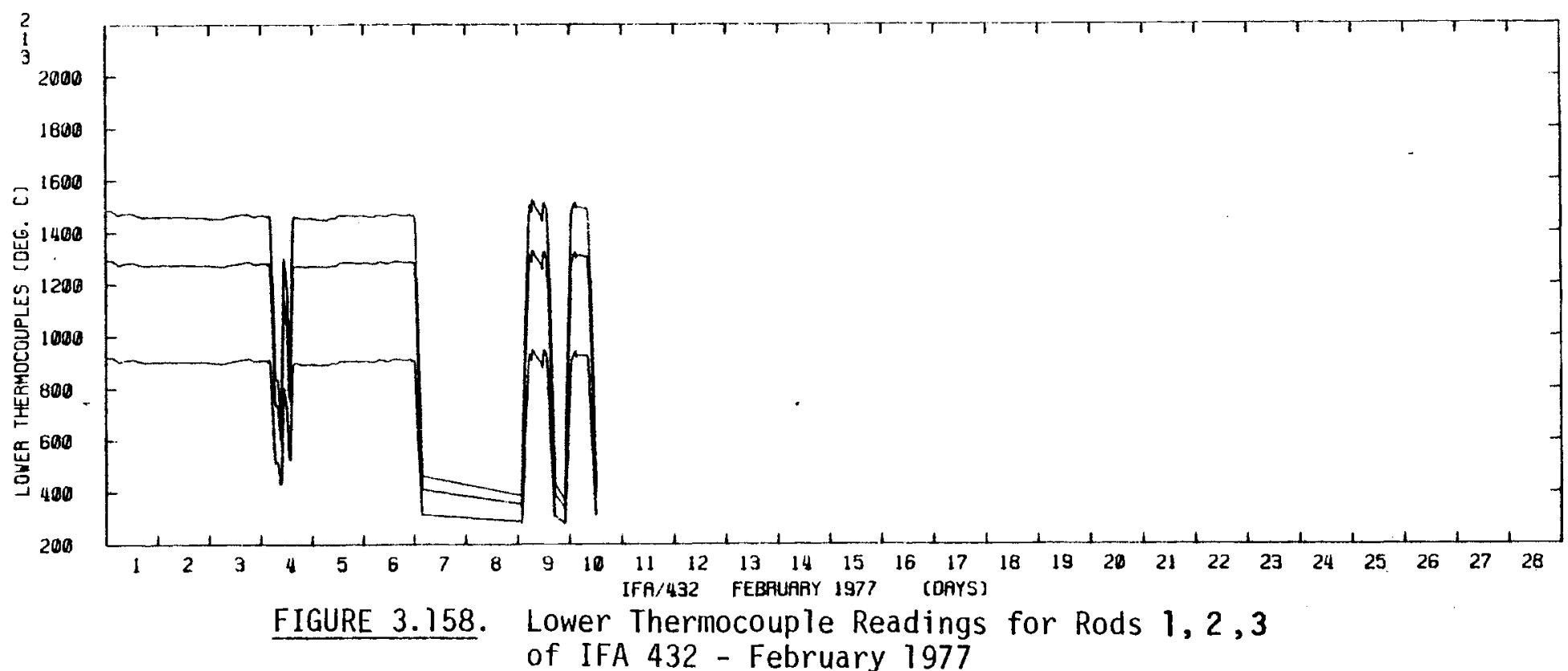




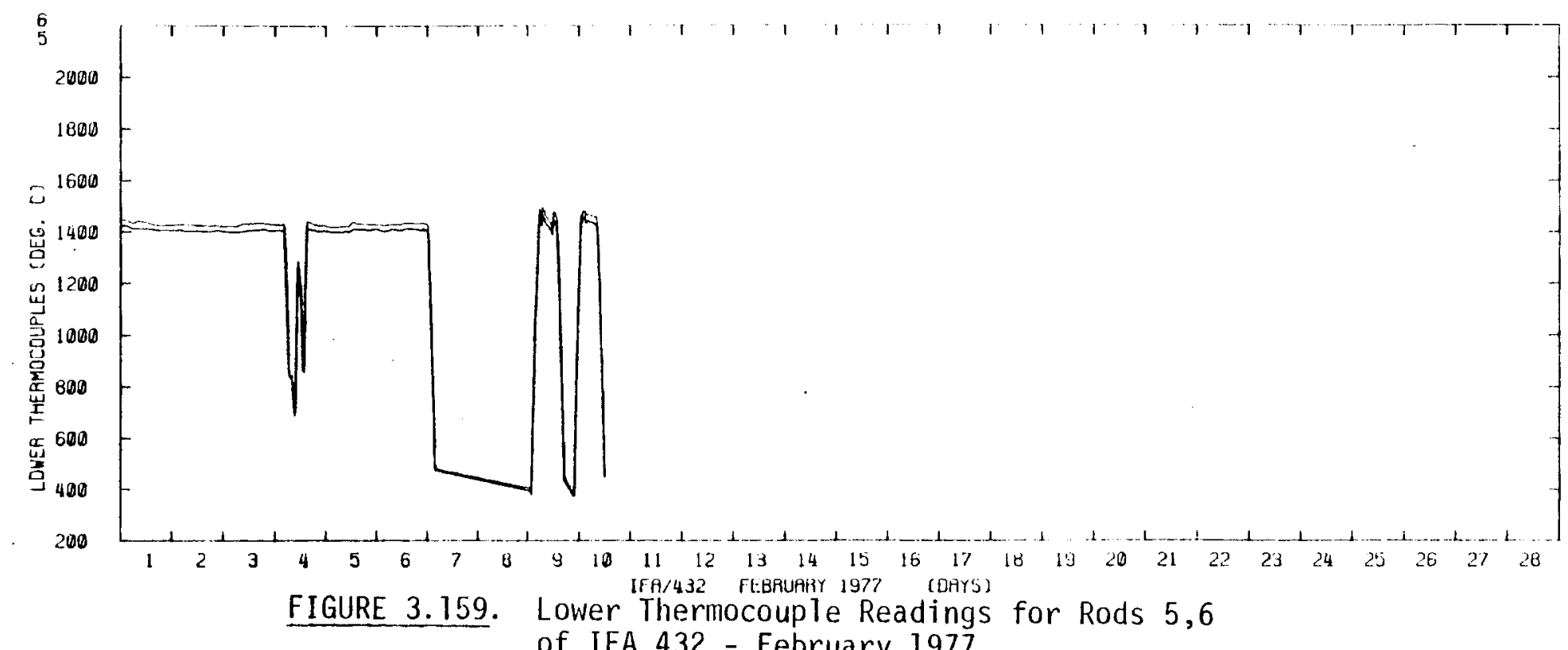

$\omega$
1
$\infty$
$N$ of IFA 432 - February 1977

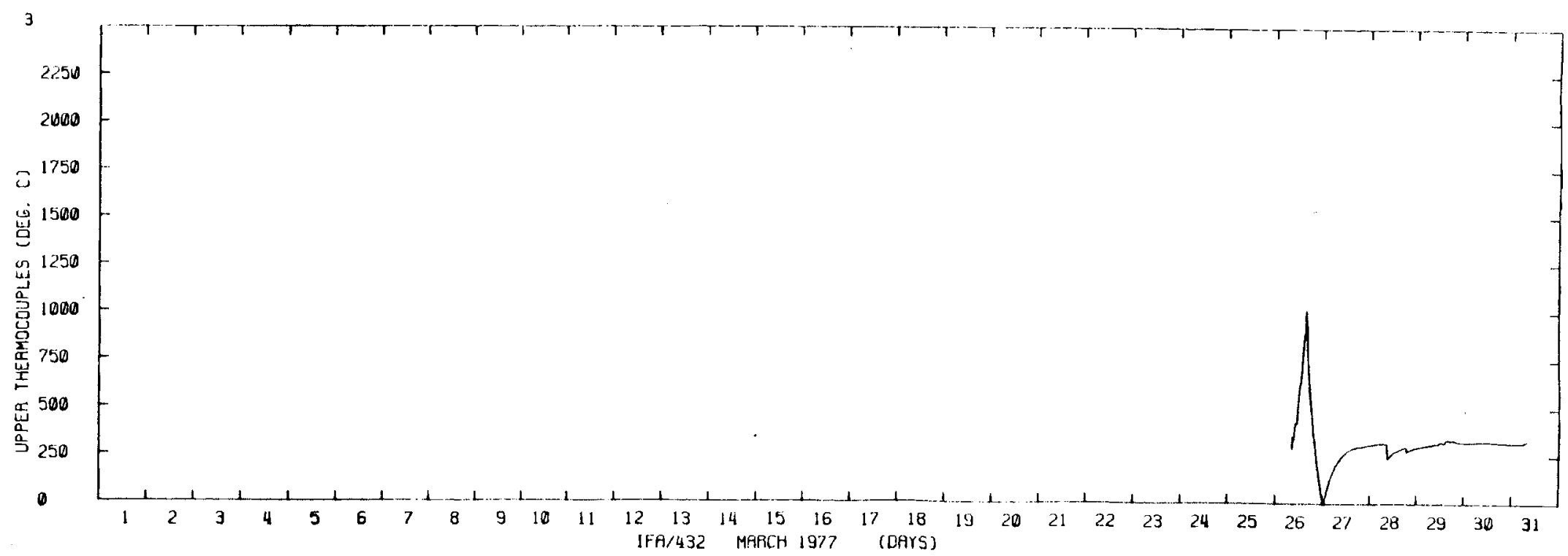

FIGURE 3.160. Upper Thermocouple Readings for Rod 3 of IFA 432 - March 1977 


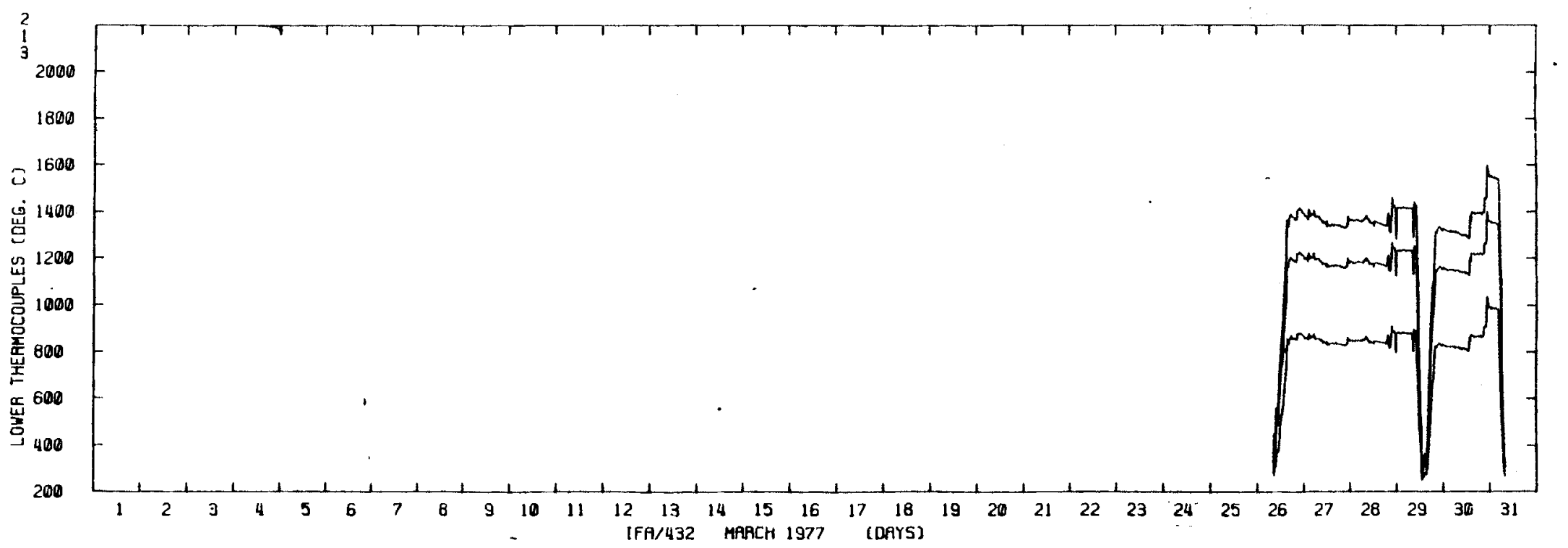

$\omega$
1
$\omega$
$\omega$

FIGURE 3.161. Lower Thermocouple Readings for Rods $1,2,3$ of IFA 432 - March 1977

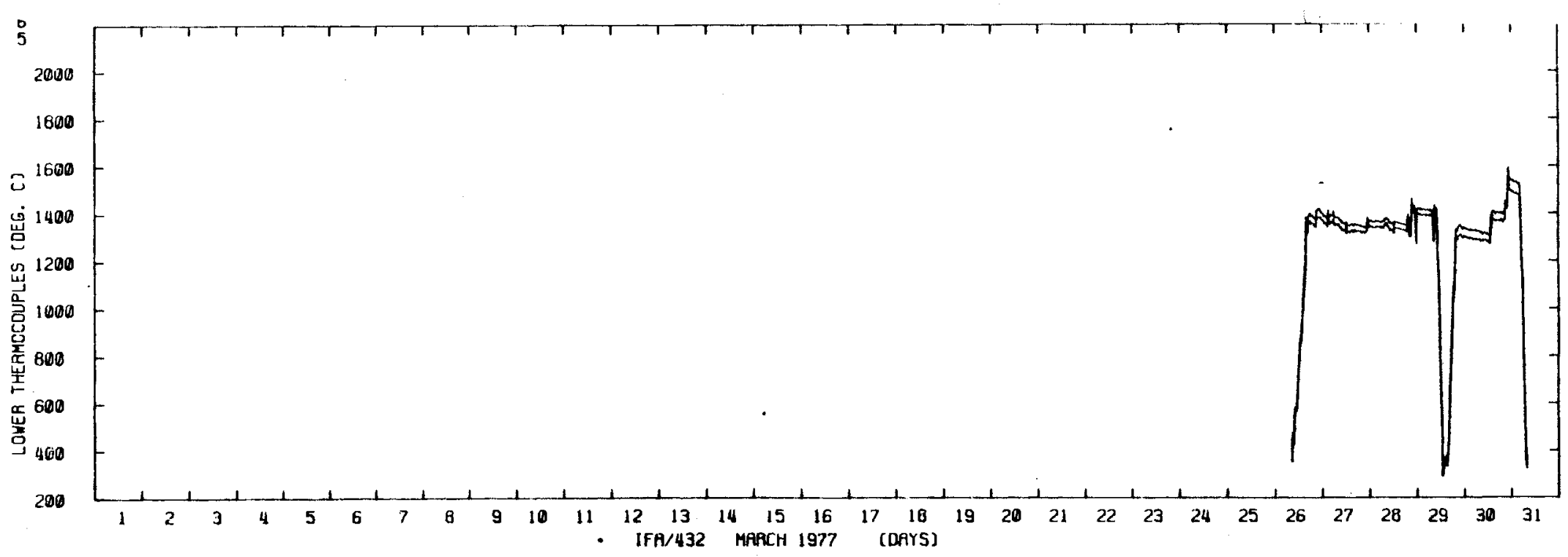

FIGURE 3.162. Lower Thermocouple Readings for Rods 5,6 of IFA 432 - March 1977 


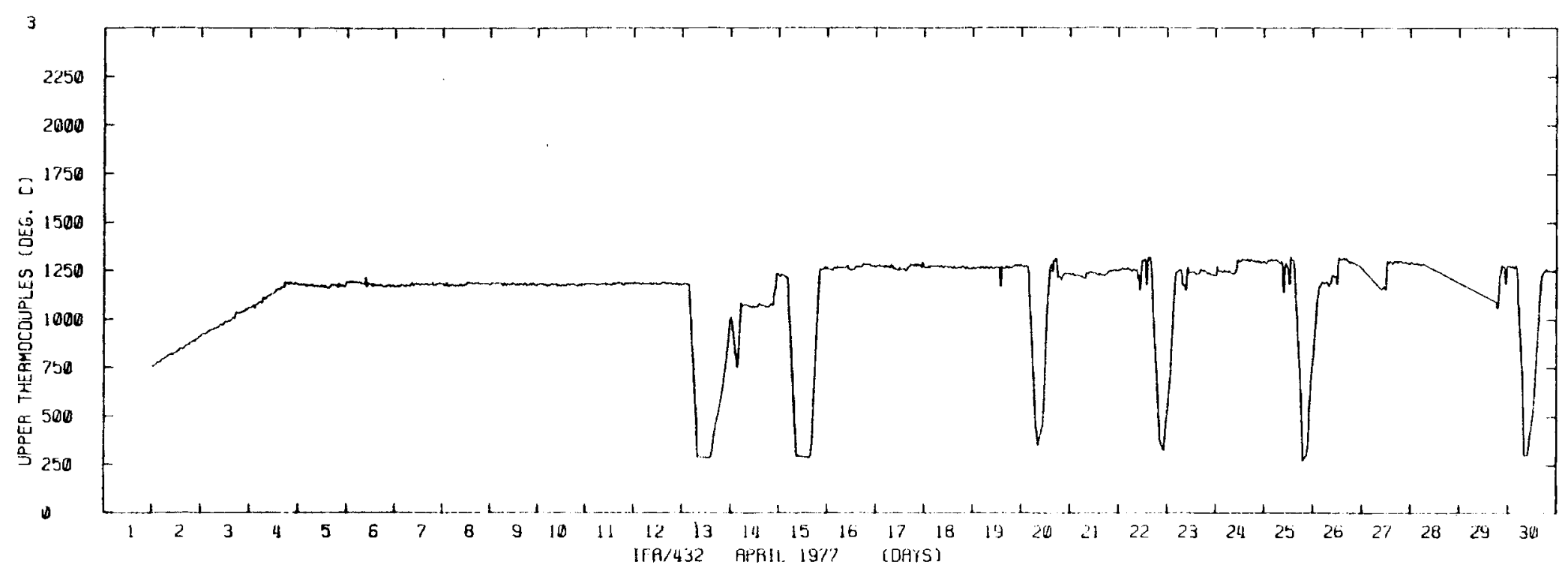

$w$
1
$\infty$
$\infty$

FIGURE 3.163. Upper Thermocouple Readings for Rod 3 of IFA 432 - April 1977

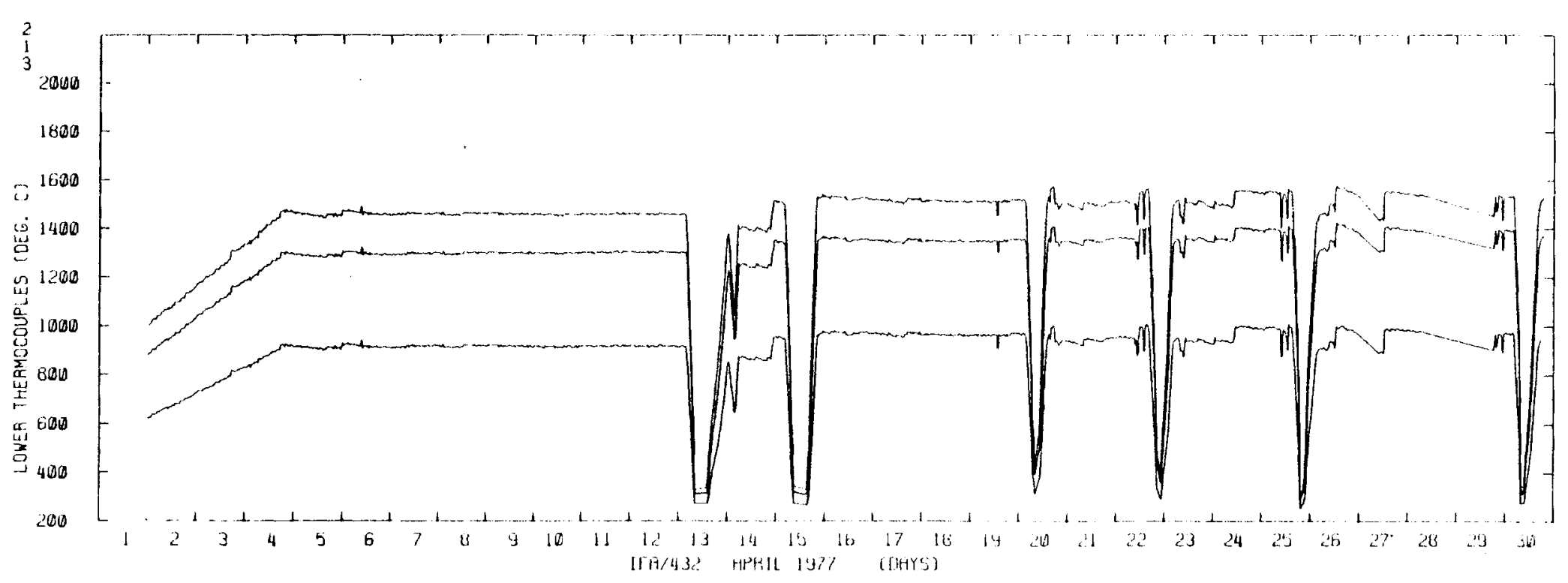

FIGURE 3.164. Lower Thermocouple Readings for Rods 1,2,3 of IFA 432 - April 1977 


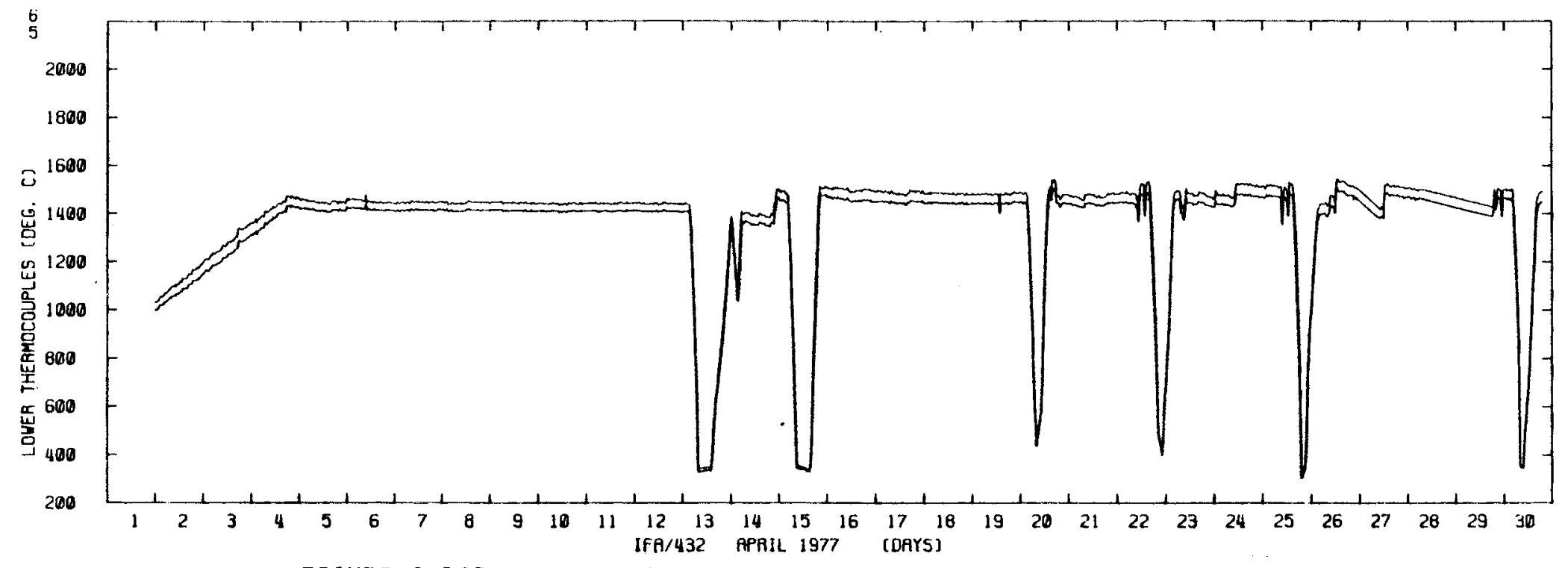

$\omega$
$\grave{1}$
o

FIGURE 3.165. Lower Thermocouple Readings for Rods 5,6 of IFA 432 - April 1977

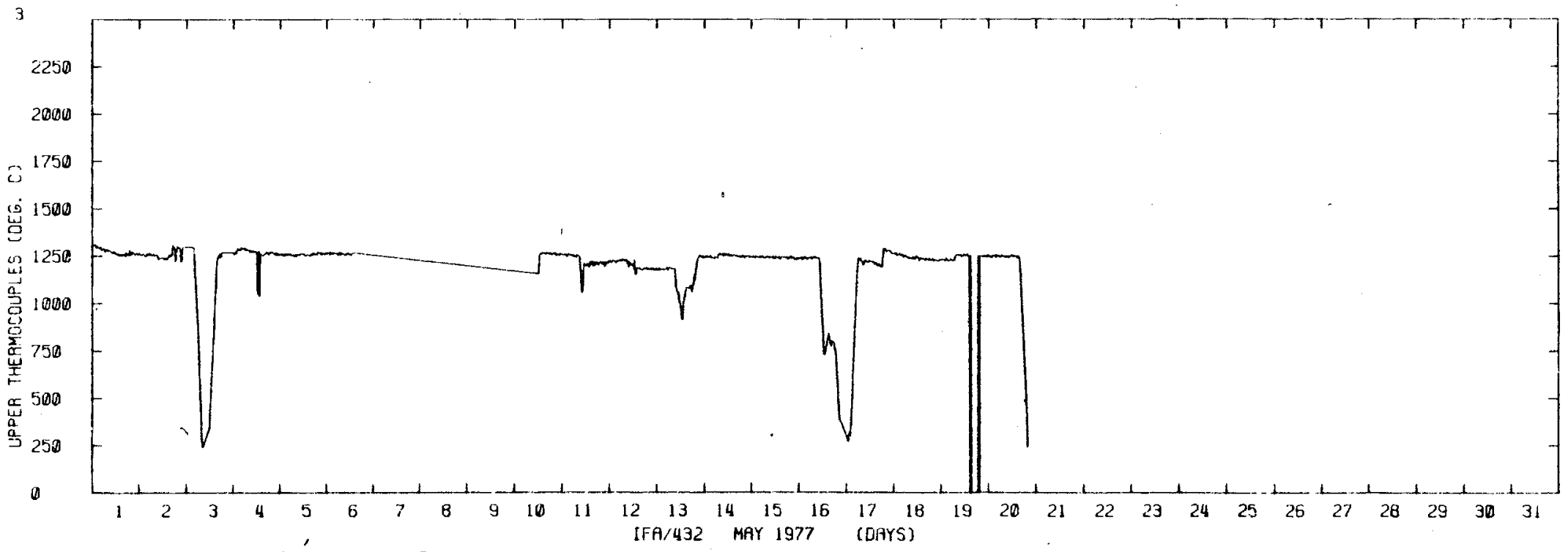

FIGURE 3.166. Upper Thermocouple Readings for Rod 3 of IFA 432 - May 1977 


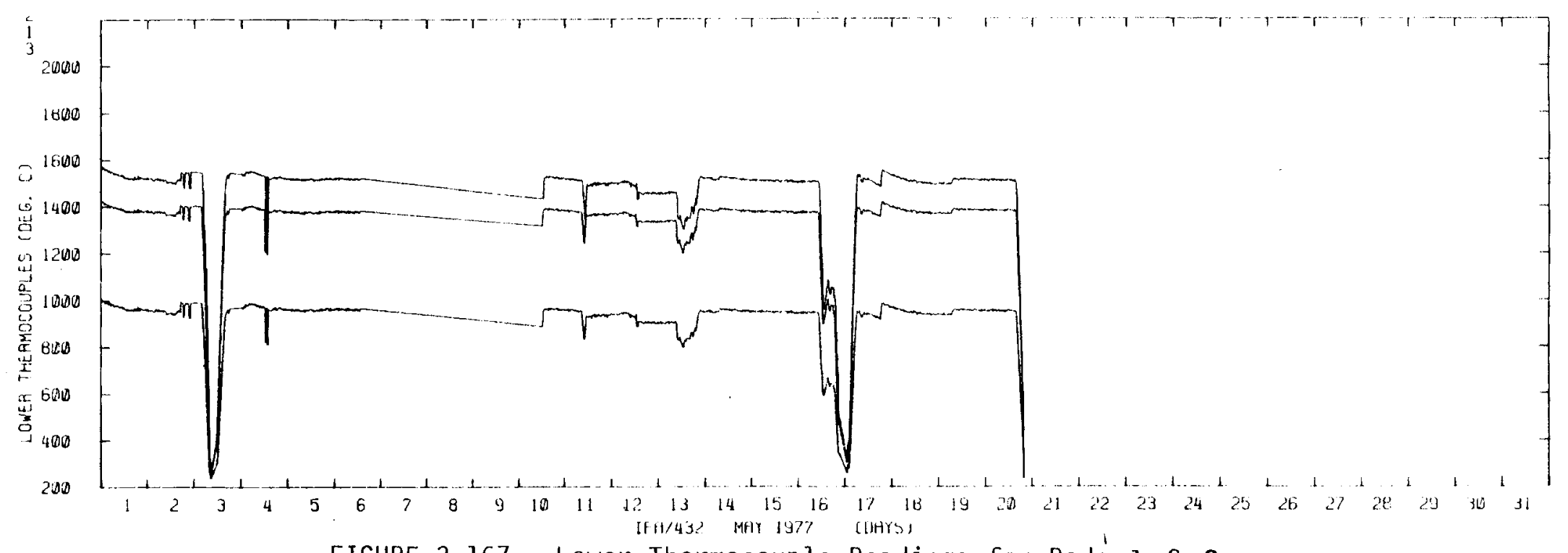

FIGURE 3.167. Lower Thermocouple Readings for Rods 1, 2,3

of IFA 432 - May 1977

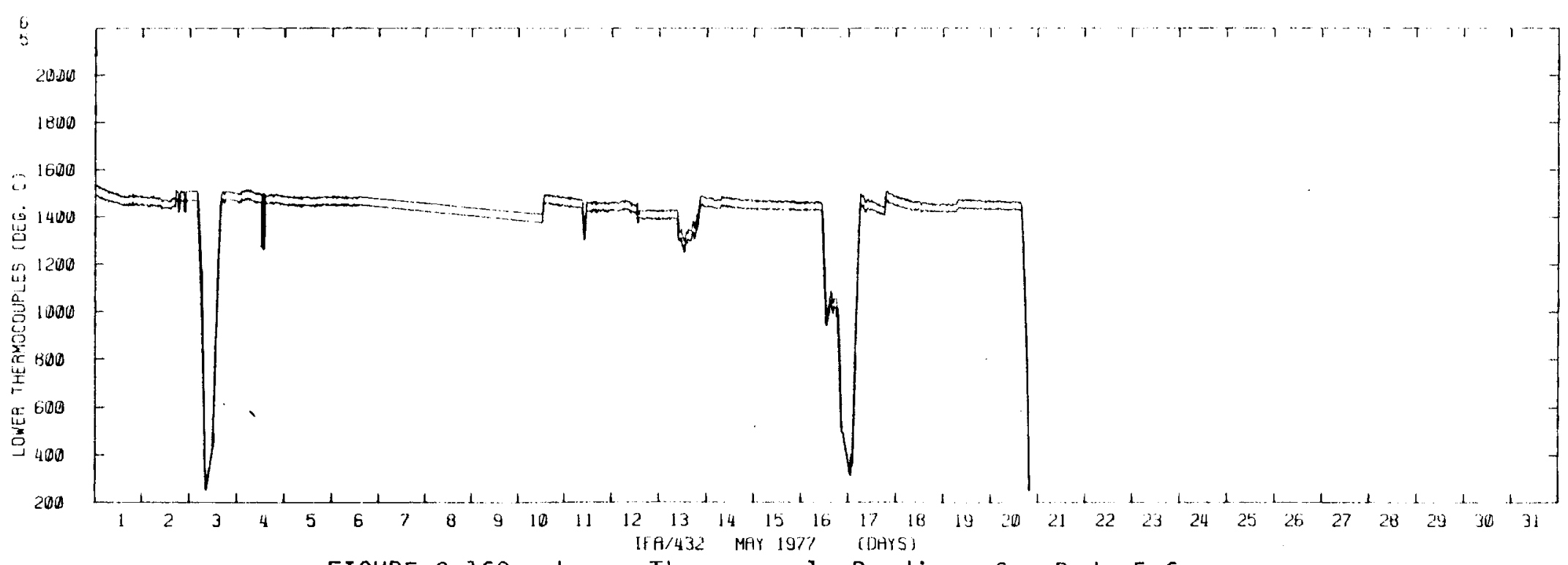

FIGURE 3.168. Lower Thermocouple Readings for Rods 5,6 of IFA 432 - May 1977 


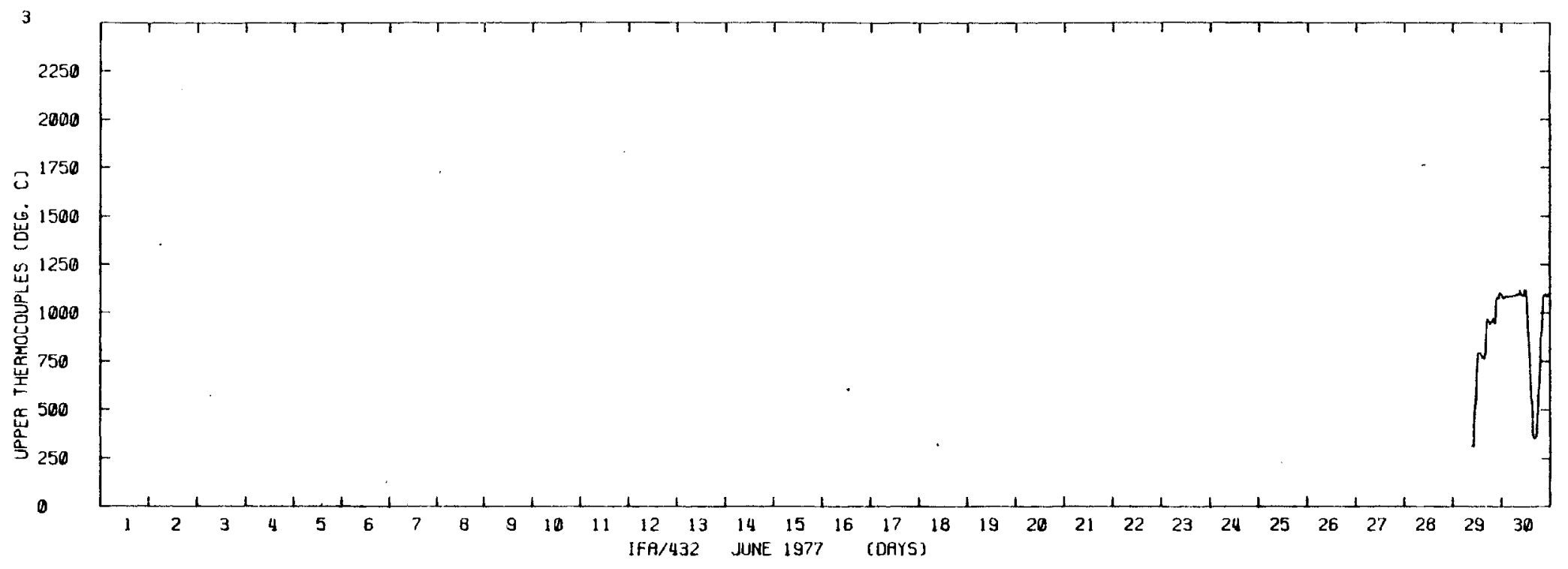

$\stackrel{\omega}{1}$

FIGURE 3.169. Upper Thermocouple Readings for Rod 3 of IFA 432 - June 1977

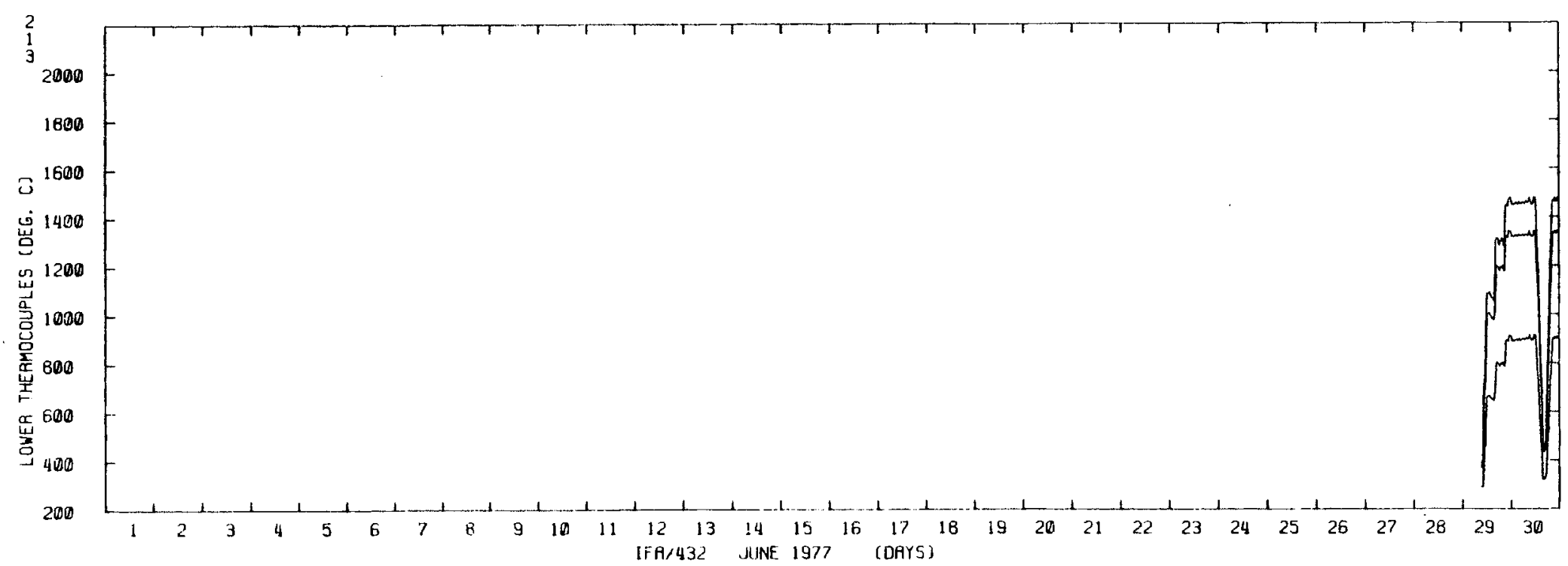

FIGURE 3.170. Lower Thermocouple Readings for Rods $1,2,3$ of IFA 432 - June 1977 


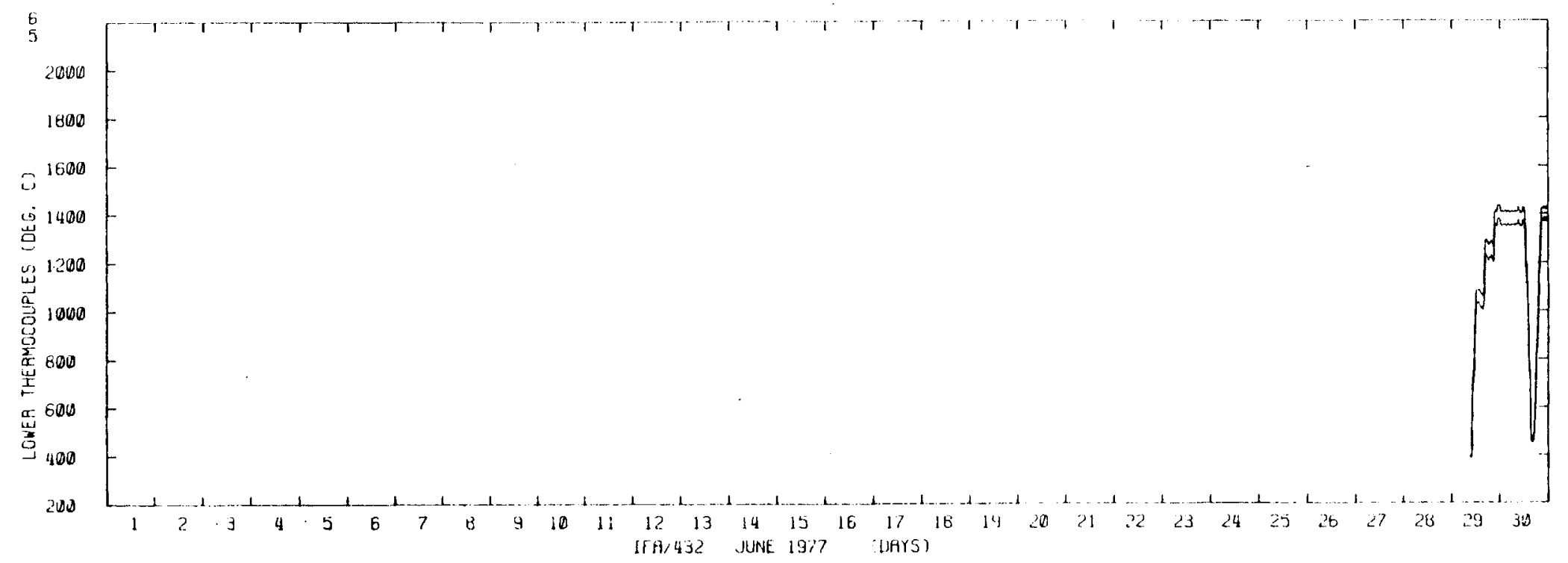

FIGURE 3.171. Lower Thermocouple Readings for Rods 5,6 of IFA 432 - June 1977

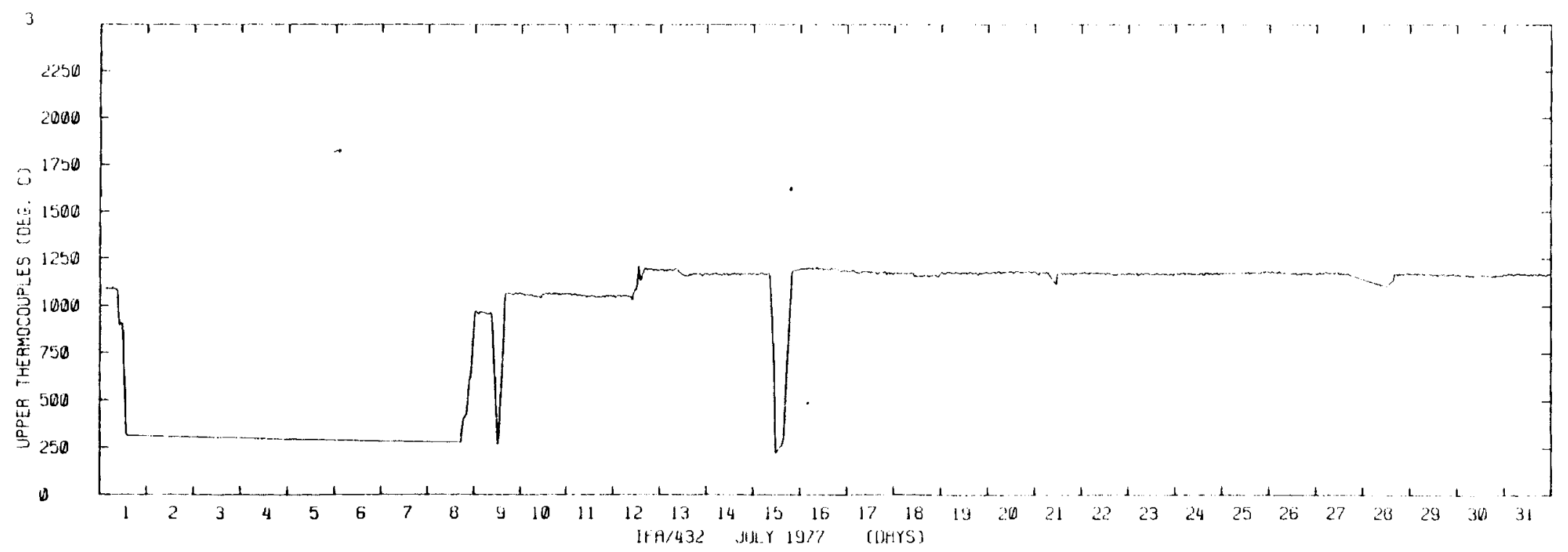

FIGURE 3.172. Upper Thermocouple Readings for Rod 3 of IFA 432 - July 1977 


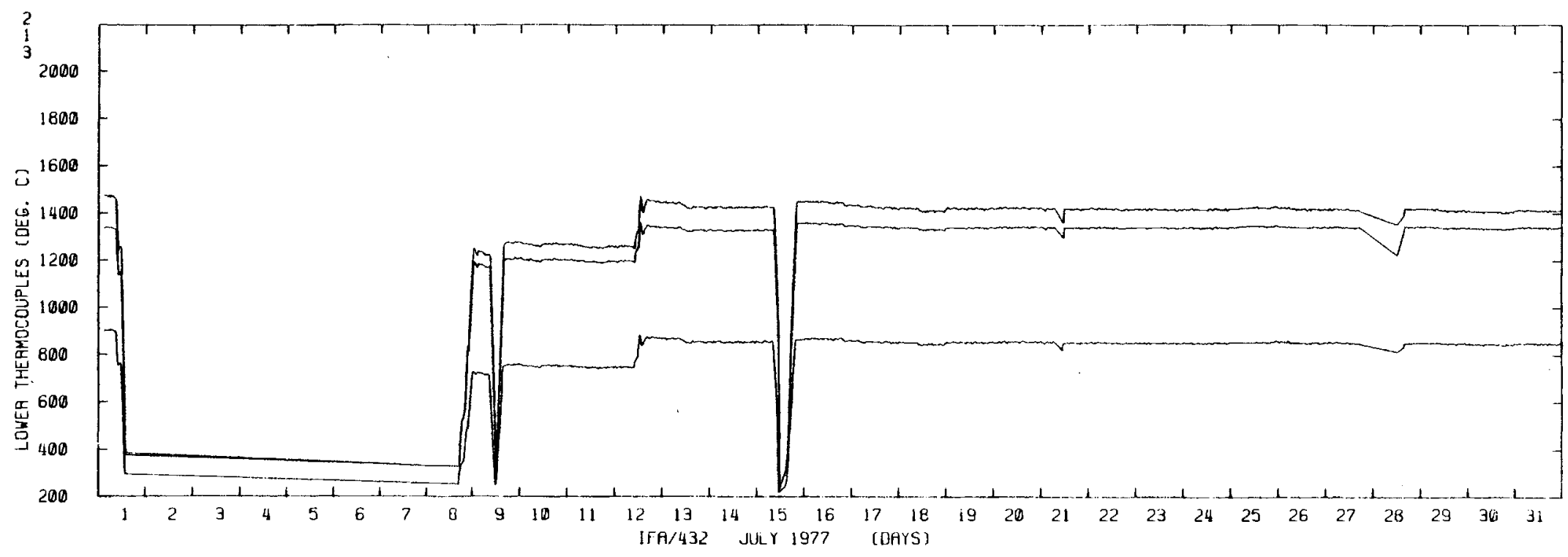

FIGURE 3.173. Lower Thermocouple Readings for Rods $1,2,3$ of IFA 432 - July 1977

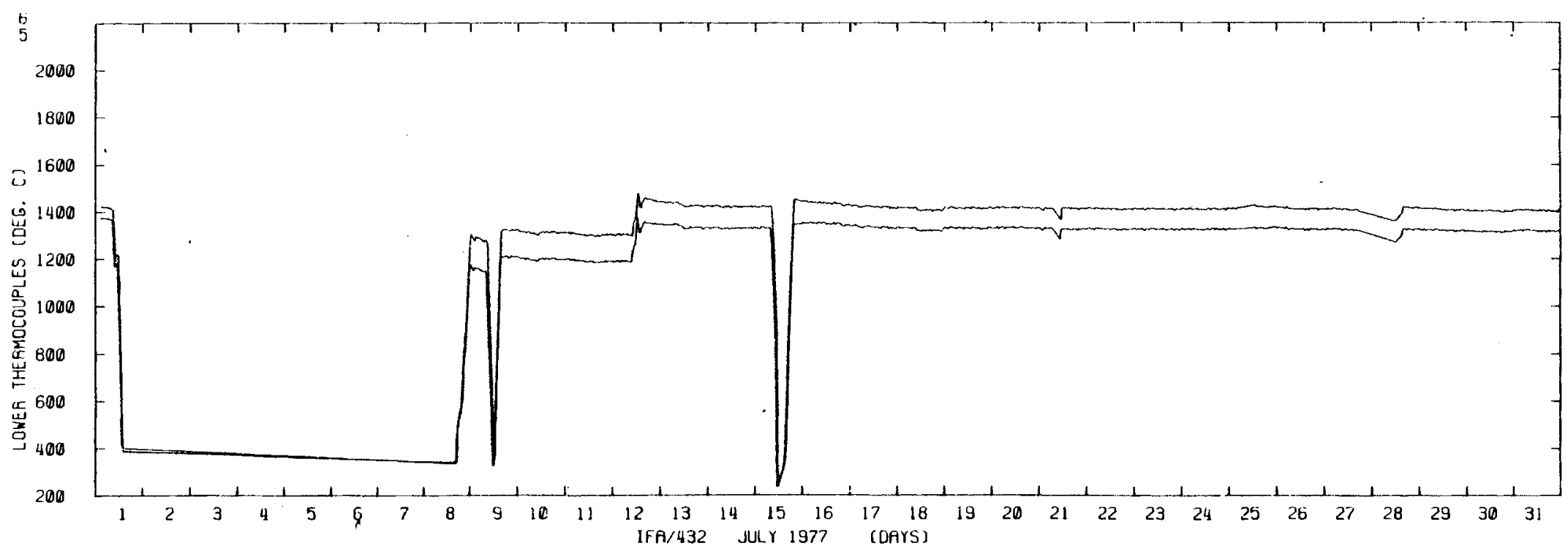

FIGURE 3.174. Lower Thermocouple Readings for Rods 5,6 of IFA 432 - July 1977 


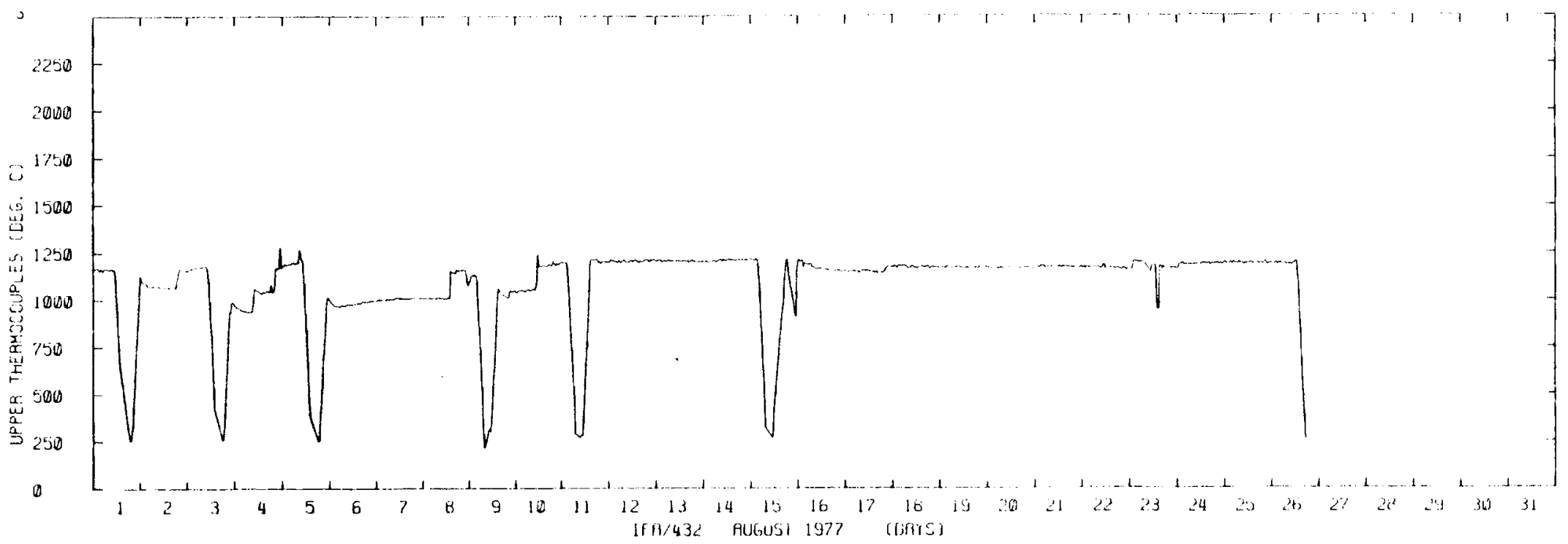

FIGURE 3.175. Upper Thermocouple Readings for Rod 3 of IFA 432 - August 1977

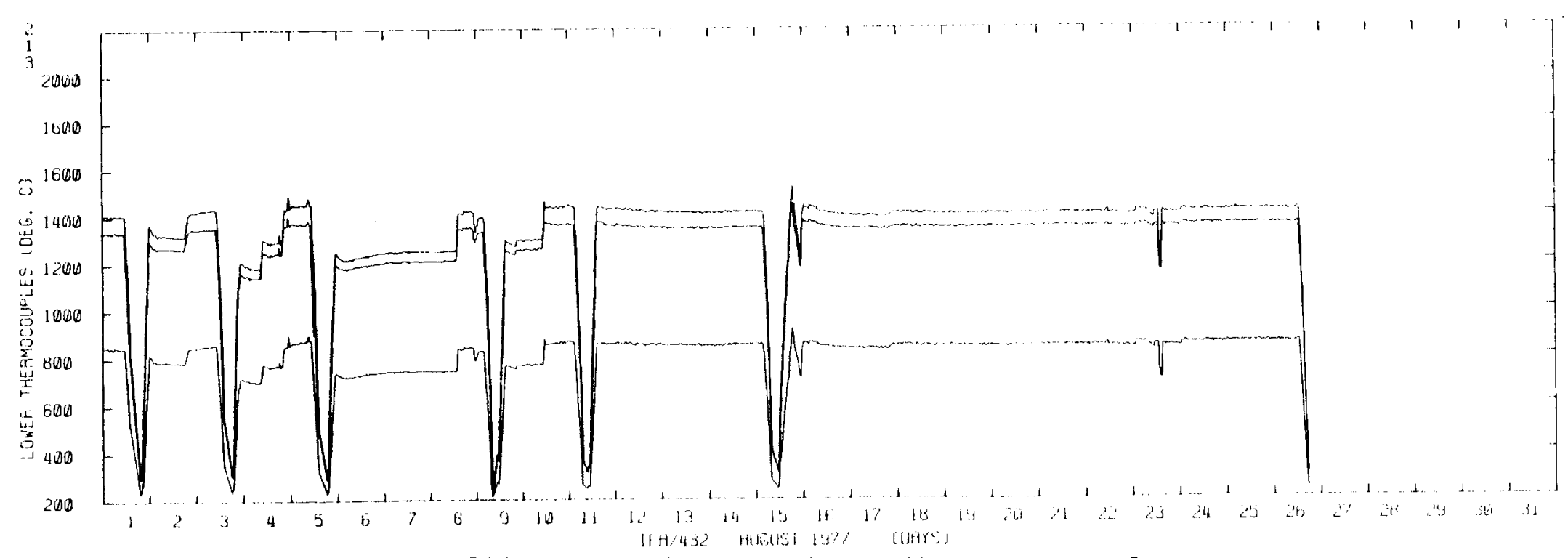

FIGURE 3.176. Lower Thermocouple Readings for Rods 1, 2,3 of IFA 432 - August 1977 


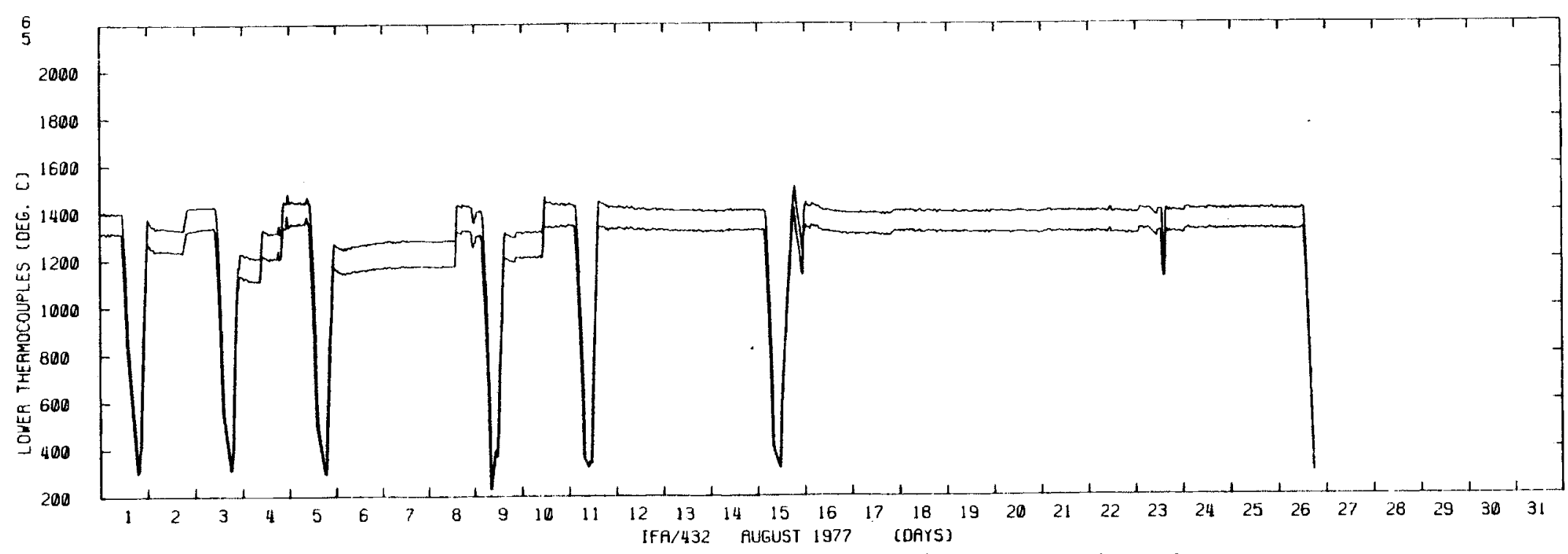

岂

FIGURE 3.177. Lower Thermocouple Readings for Rods 5,6 of IFA 432 - August 1977

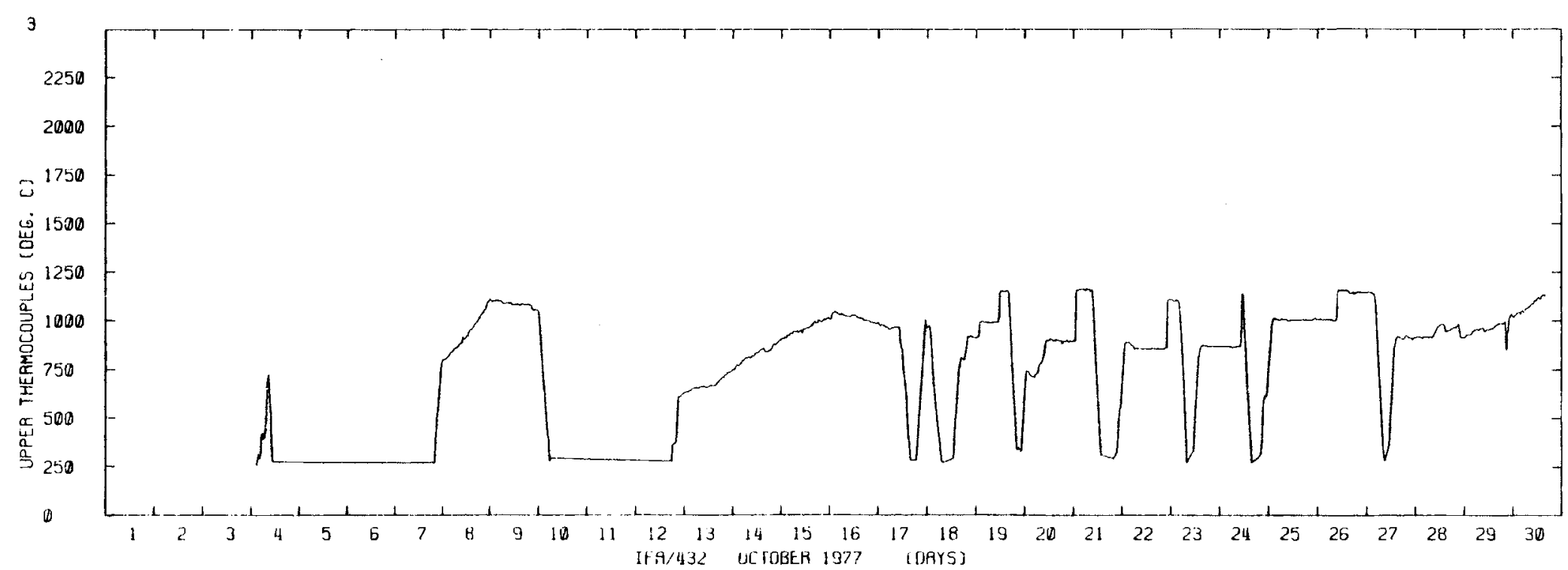

FIGURE 3.178. Upper Thermocouple Readings for Rod 3 of IFA 432 - October 1977 


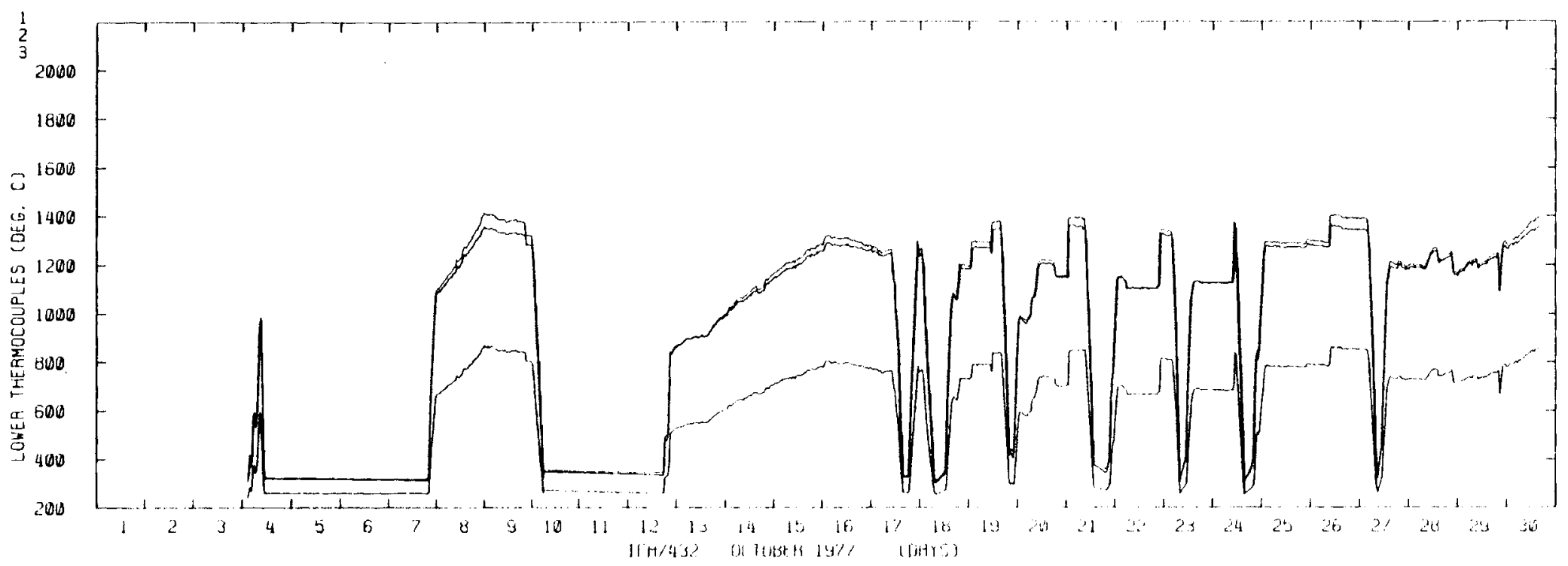

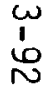

FIGURE 3.179. Lower Thermocouple Readings for Rods 1,2,3 of IFA 432 - October 1977

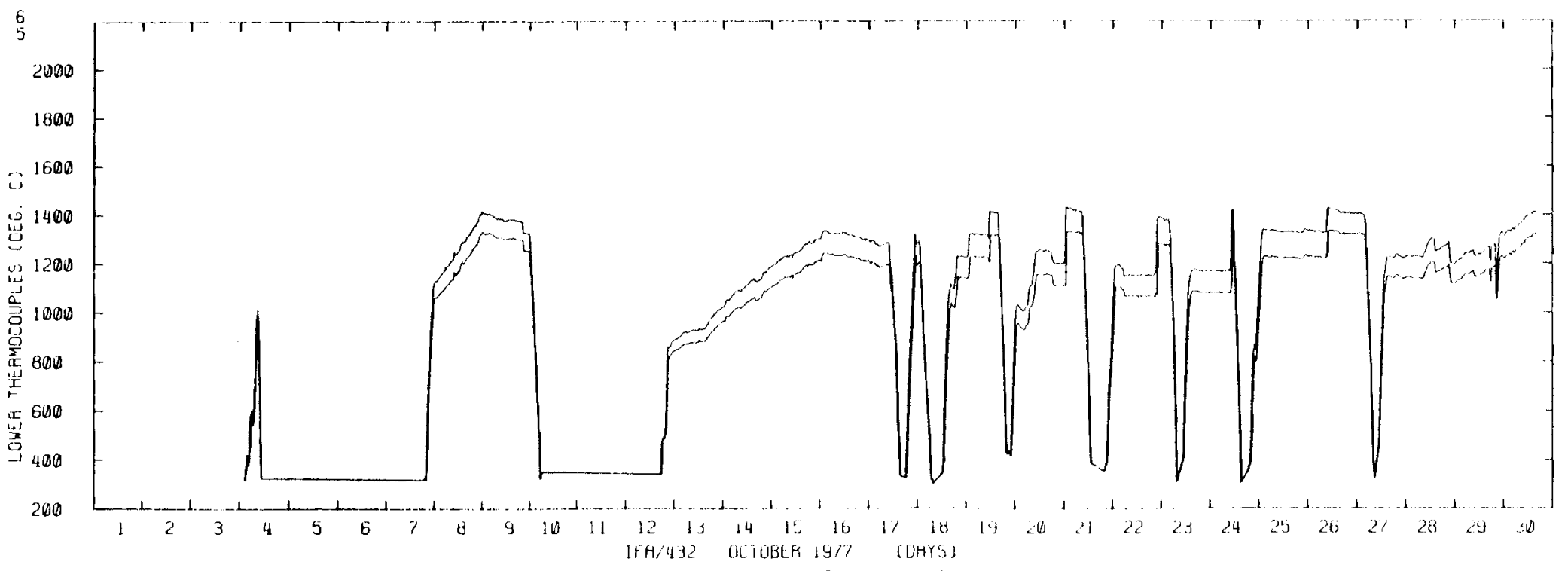

FIGURE 3.180. Lower Thermocouple Readings for Rods 5,6 of IFA 432 - October 1977 


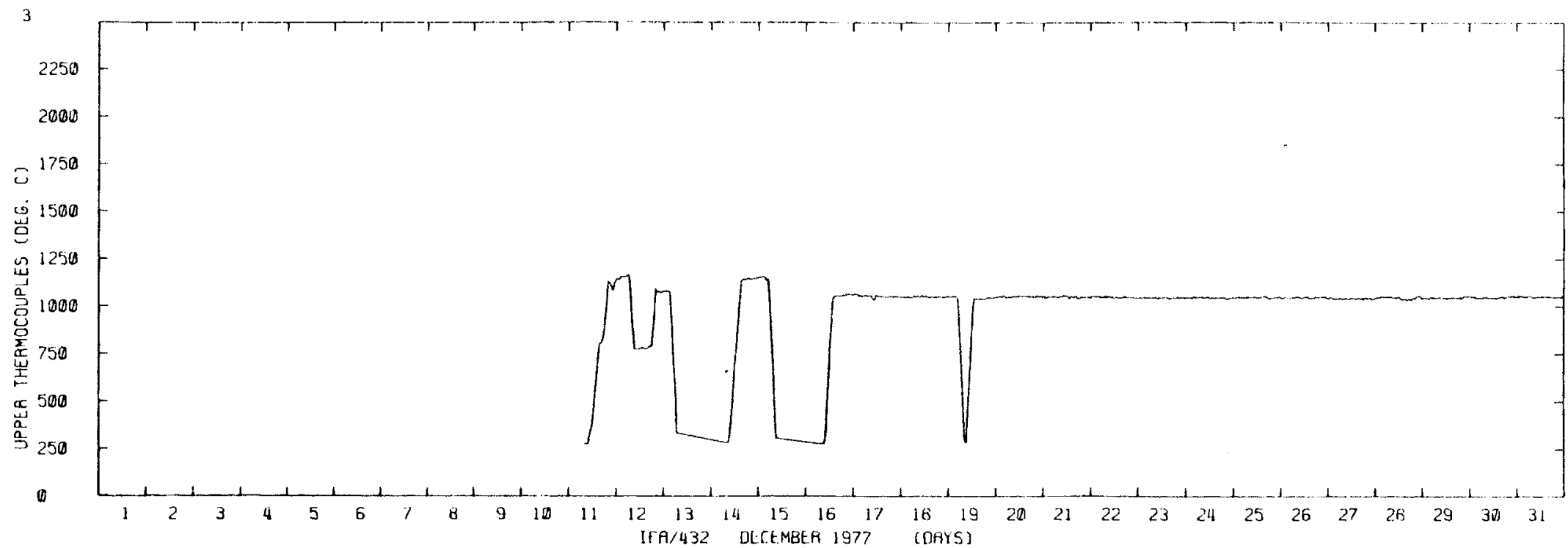

FIGURE 3.181. Upper Thermocouple Readings for Rod 3 of IFA 432 - December 1977

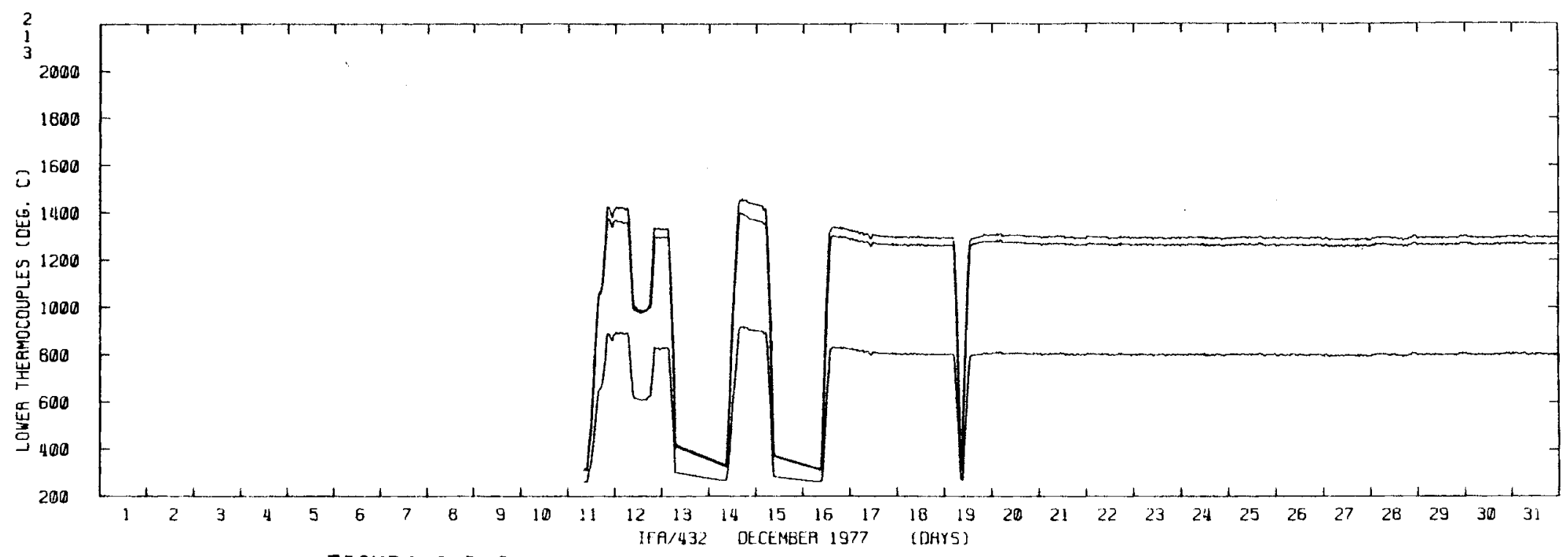

FIGURE 3.182. Lower Thermocouple Readings for Rods 1,2,3 of IFA 432 - December 1977 


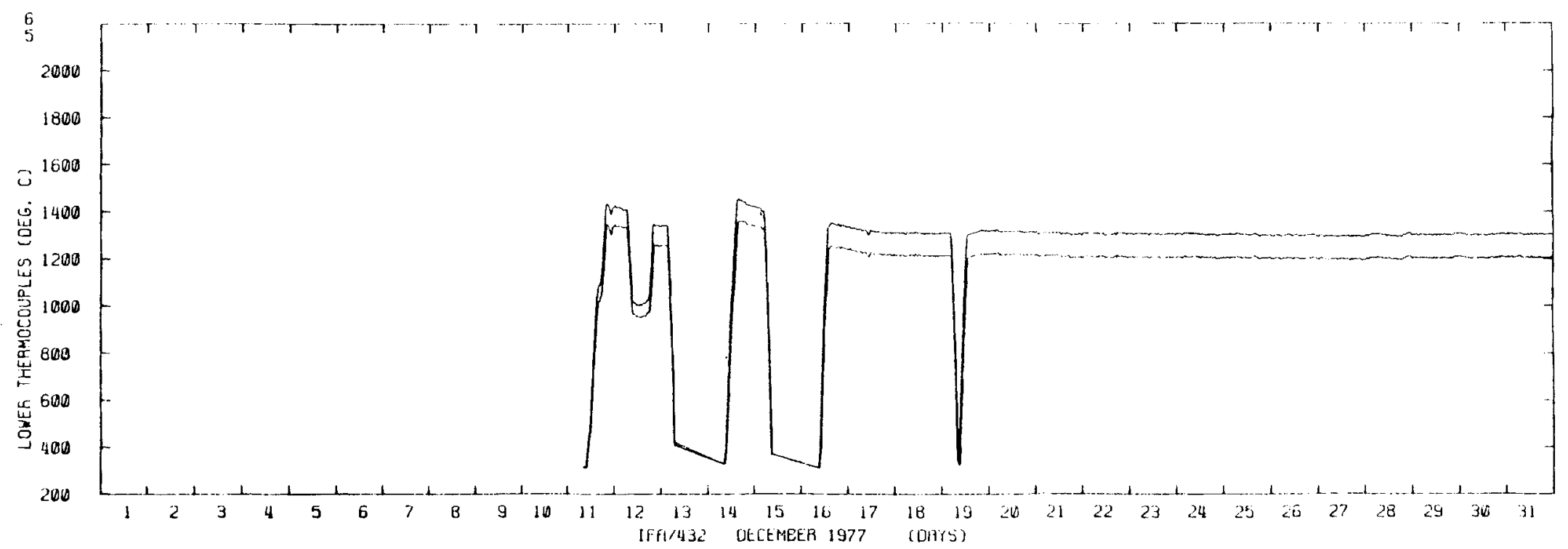

$w$
1
$\dot{p}$

FIGURE 3.183. Lower Thermocouple Readings for Rods 5,6 of IFA 432 - December 1977

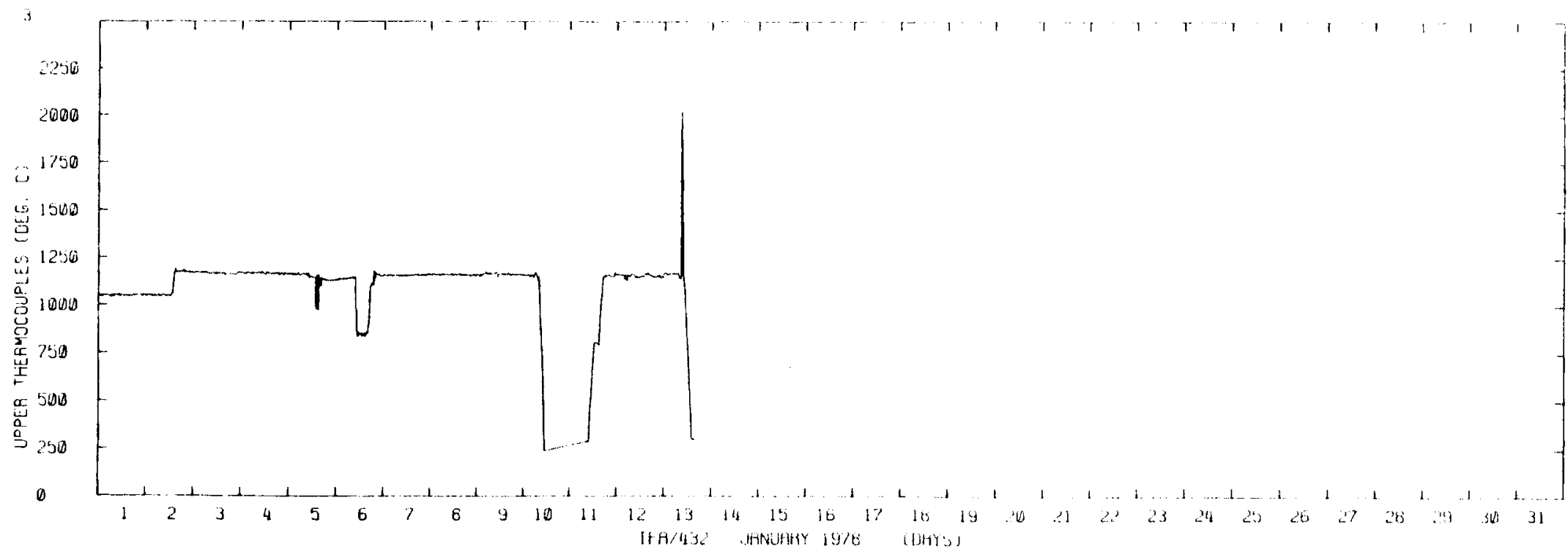

FIGURE 3.184. Upper Thermocouple Readings for Rod 3 of IFA 432 - January 1978 


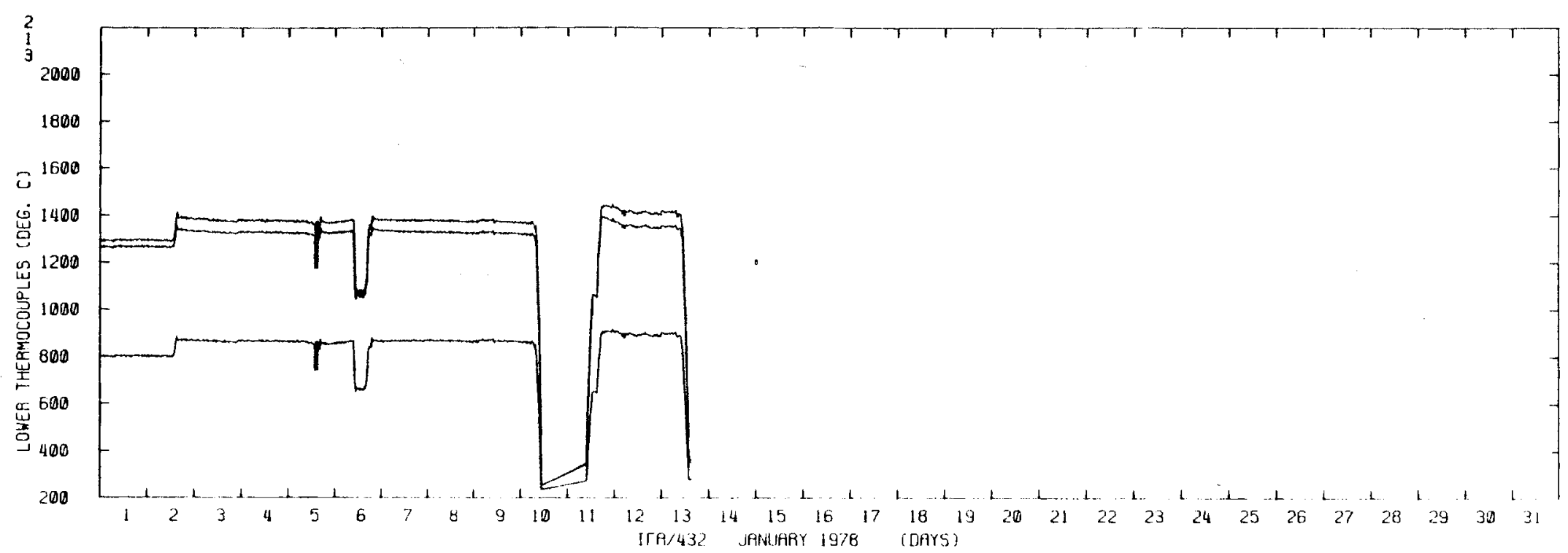

$\omega$
$\vdots$
0
$o$

FIGURE 3.185. Lower Thermocouple Readings for Rods 1, 2, 3 of IFA 432 - January 1978

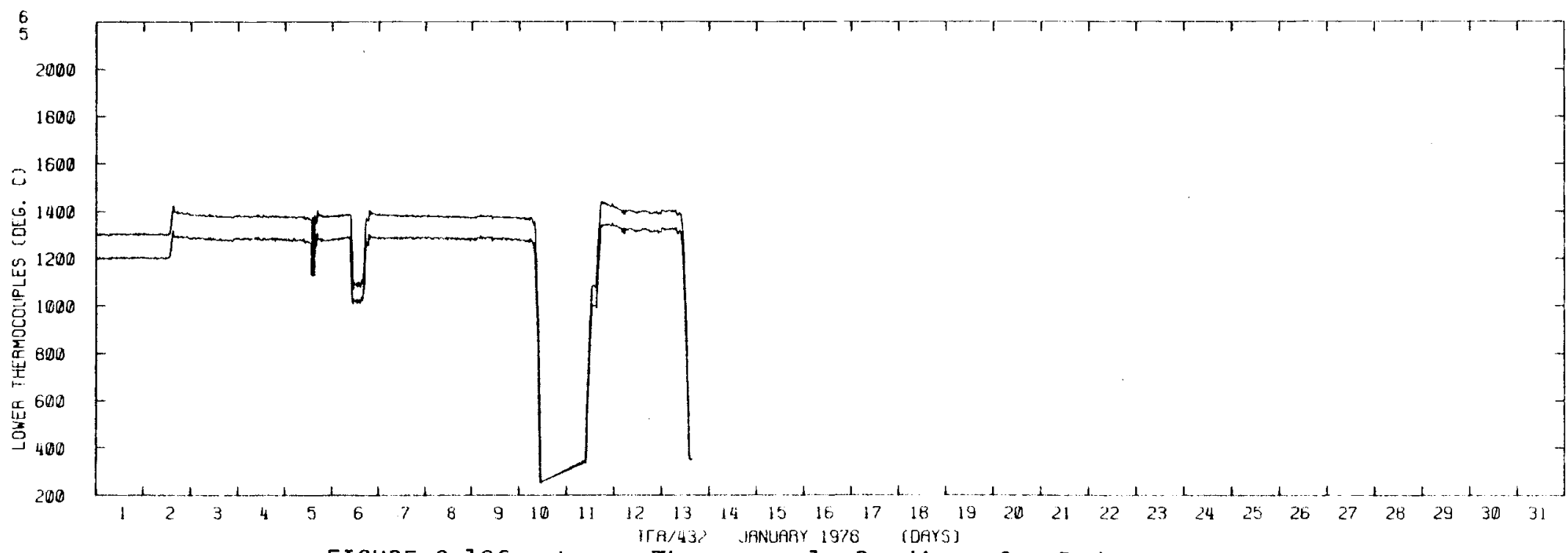

FIGURE 3.186. Lower Thermocouple Readings for Rods 5,6 of IFA 432 - January 1978 


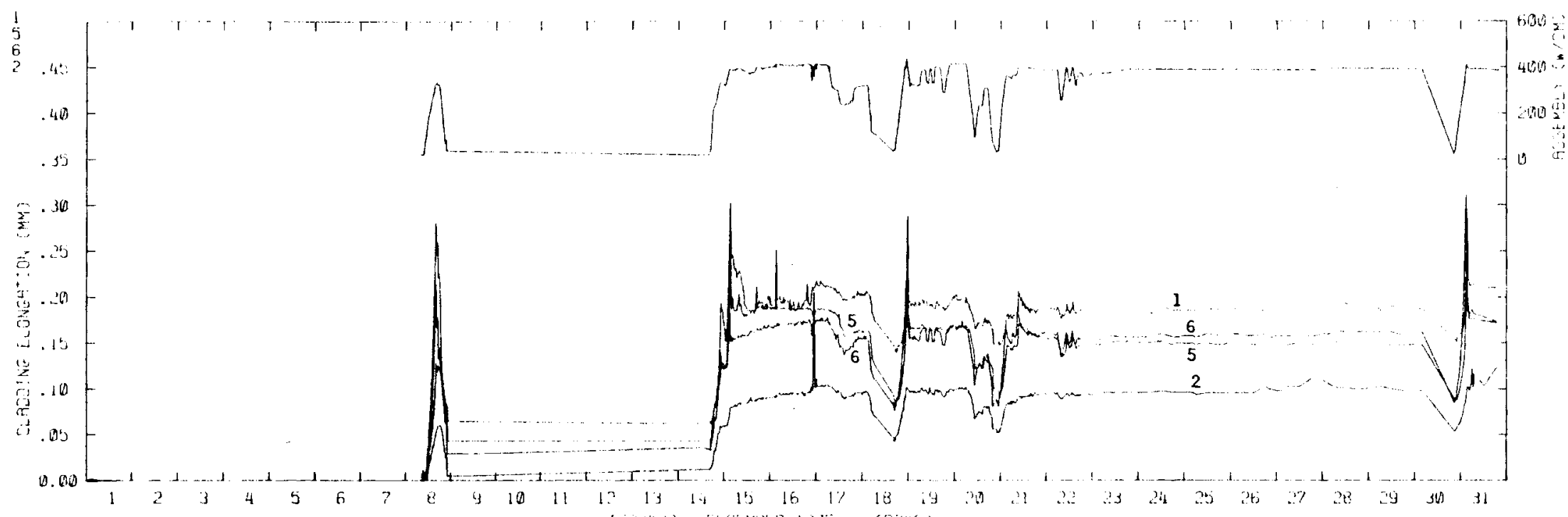

$\omega$
1
0

FIGURE 3.187. Cladding Elongation Sensor Readings for Rods 1, 2, 5, 6 of IFA 432 - December 1975

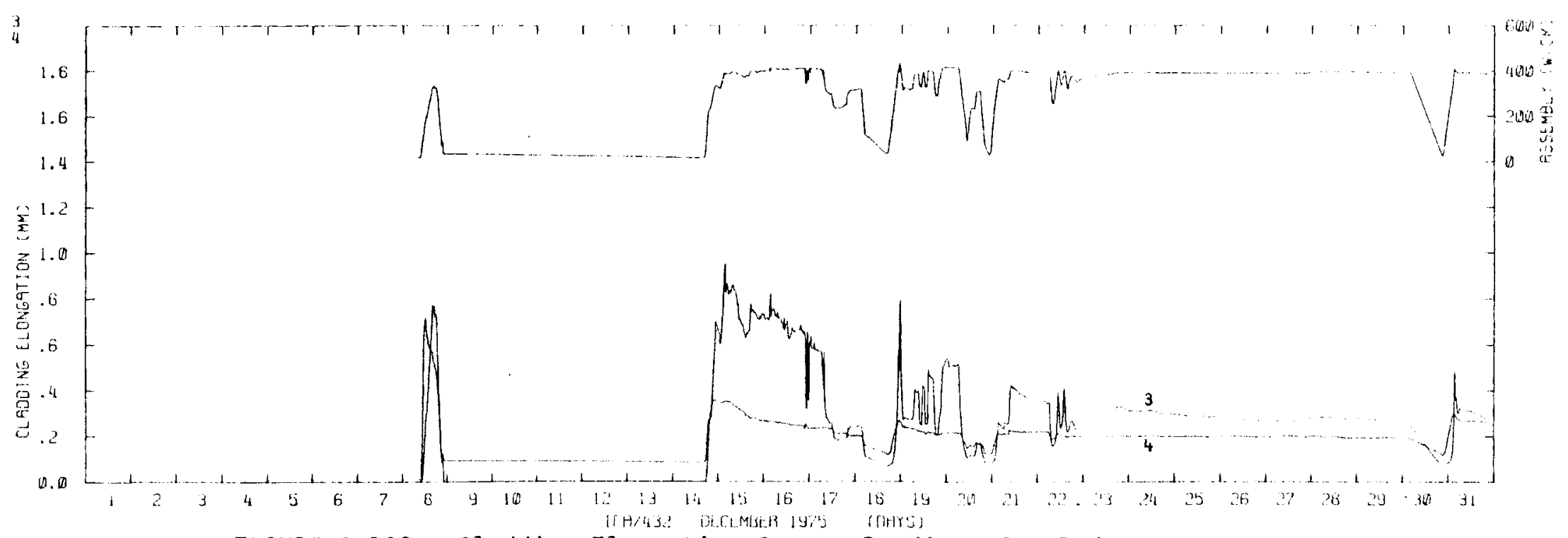

FIGURE 3.188. Cladding Elongation Sensor Readings for Rods 3, 4 of IFA 432 - December 1975 


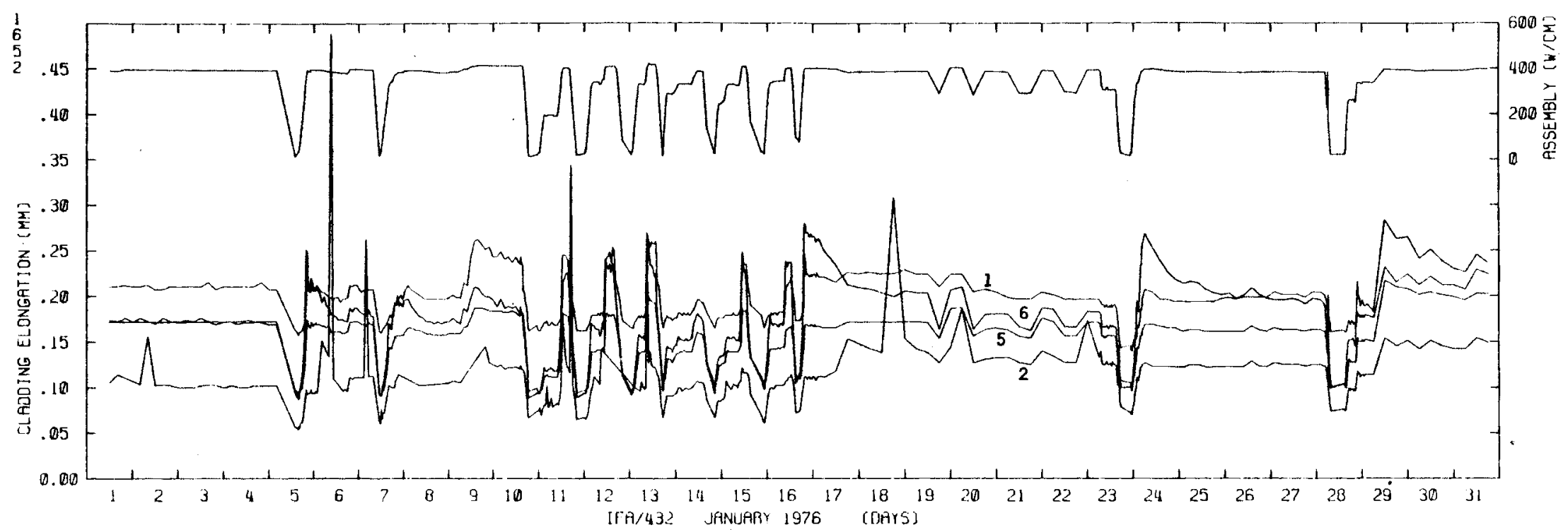

$\omega$
1
$\dot{1}$

FIGURE 3.189. Cladding Elongation Sensor Readings for Rods 1, 2, 5, 6 of IFA 432 - January 1976

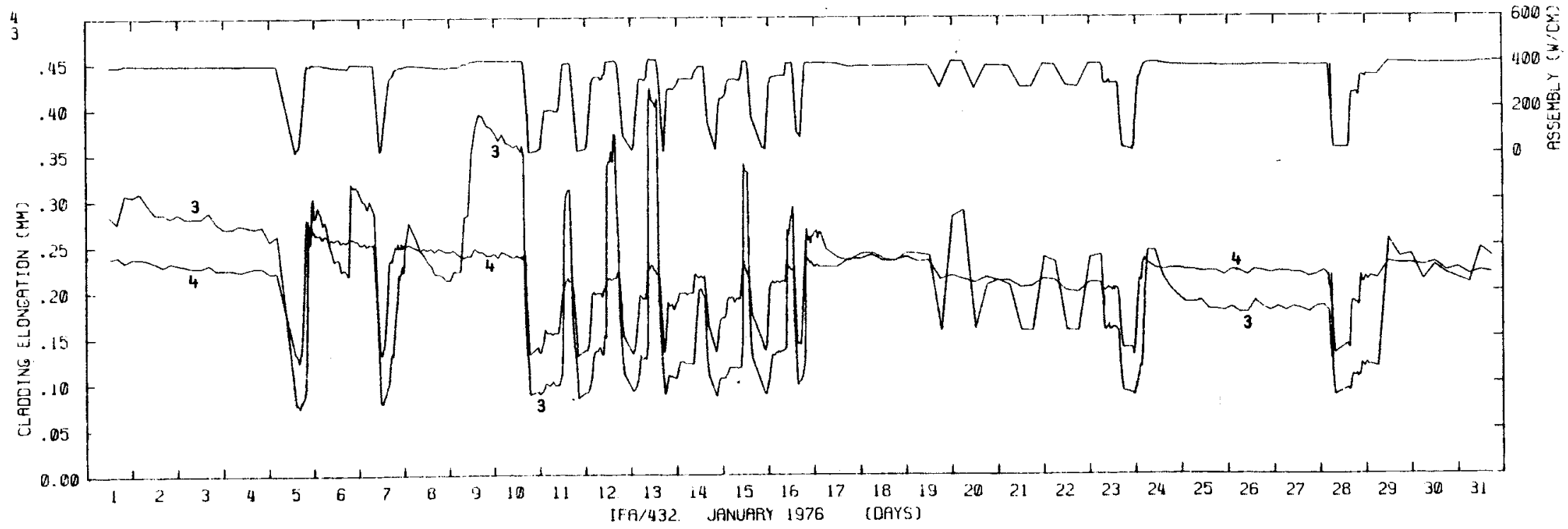

FIGURE 3.190. Cladding Elongation Sensor Readings for Rods 3, 4 of IFA 432 - January 1976 


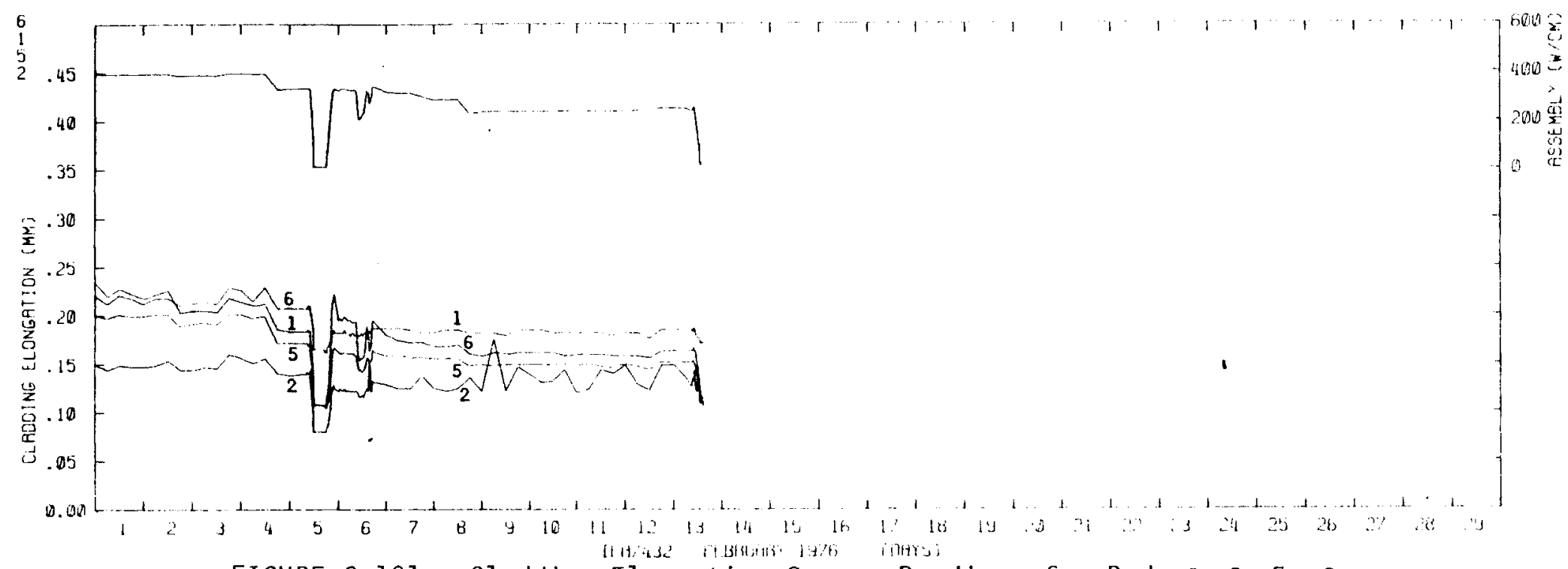

FIGURE 3.191. Cladaing Elongation Sensor Readings for Rods 1, 2, 5,6 of IFA 432 - February 1976

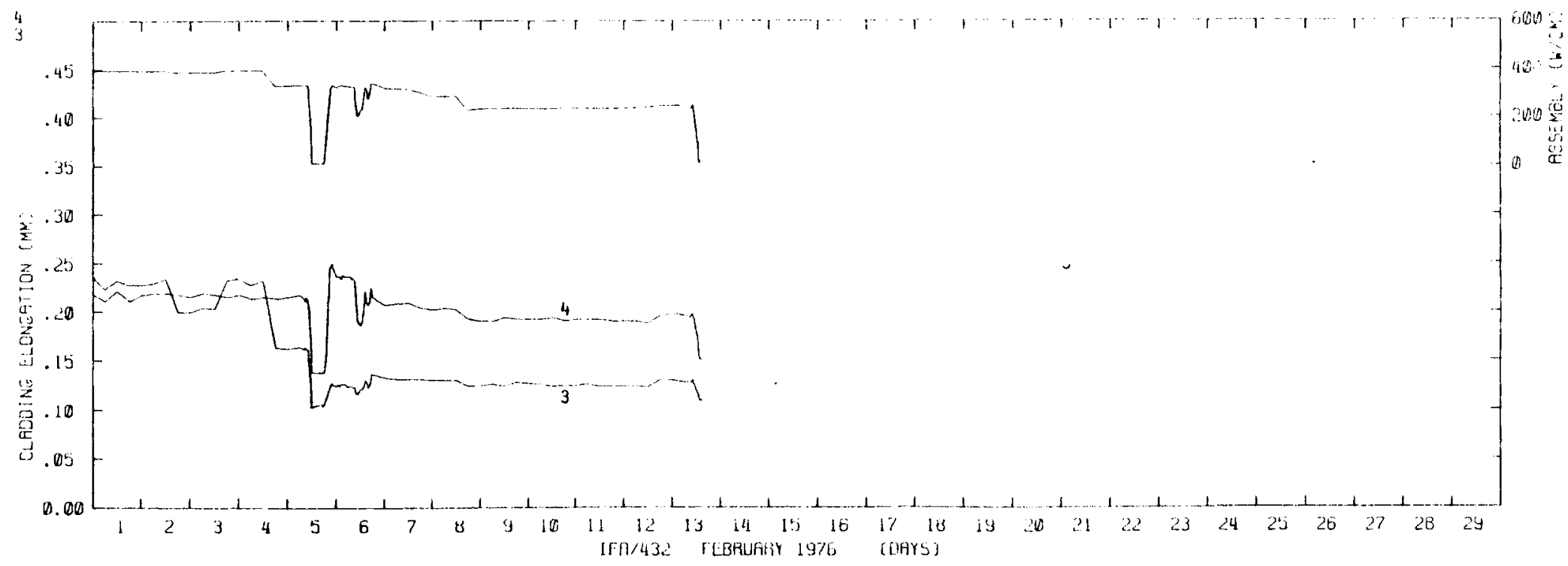

FIGURE 3.192. Cladding Elongation Sensor Readings for Rods 3, 4 of IFA 432 - February 1976 


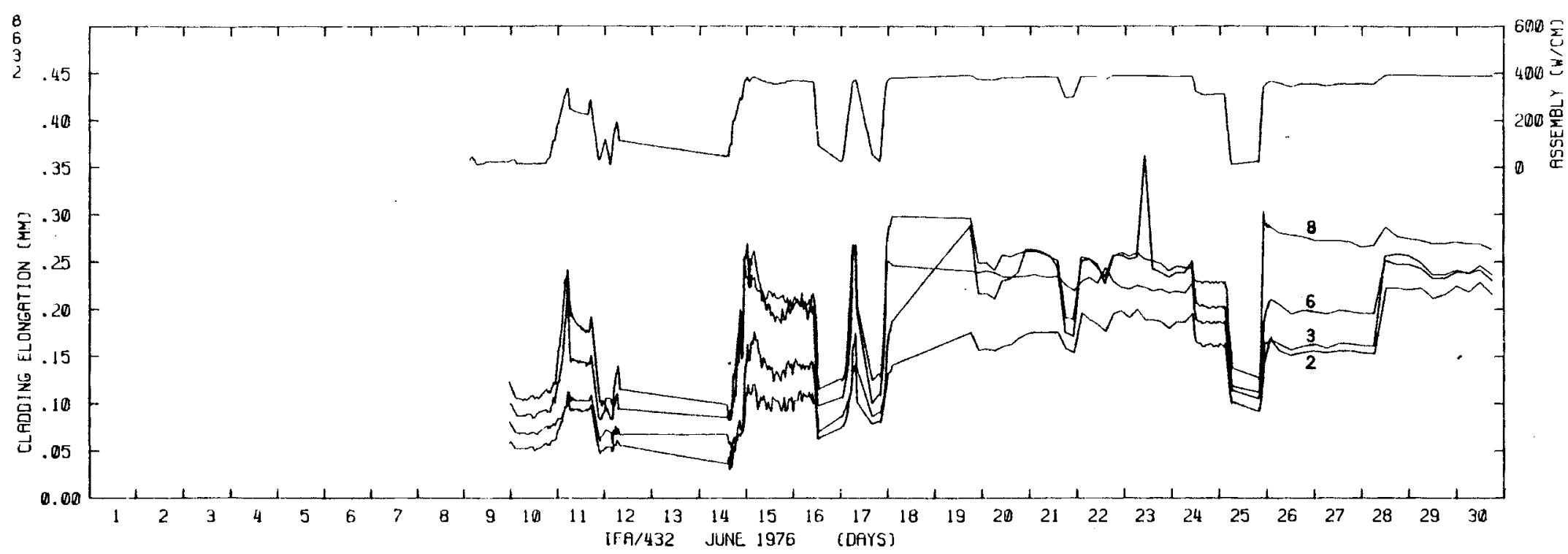

1
1
8

FIGURE 3.193. Cladding Elongation Sensor Readings for Rods 2, 3, 6, 8 of IFA 432 - June 1976

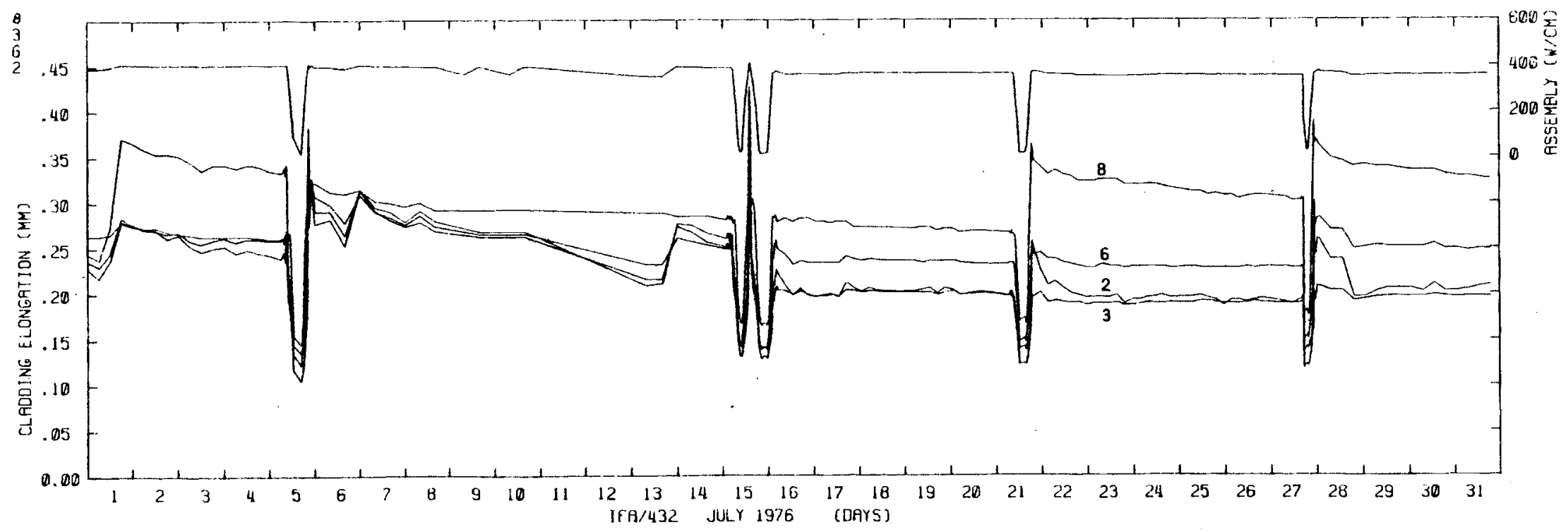

FIGURE 3.194. Cladding Elongation Sensor Readings for Rods 2, 3, 6, 8 of IFA 432 - July 1976 


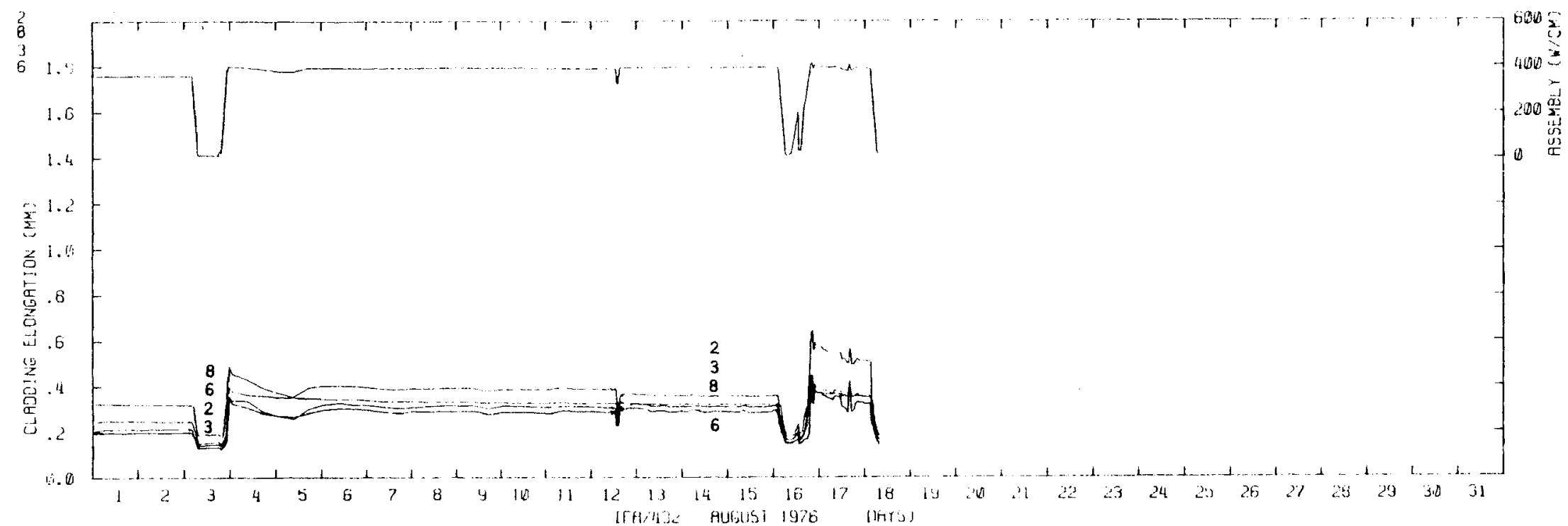

FIGURE 3.195. Cladding Elongation Sensor Readings for Rods 2, 3, 6, 8 of IFA 432 - August 1976

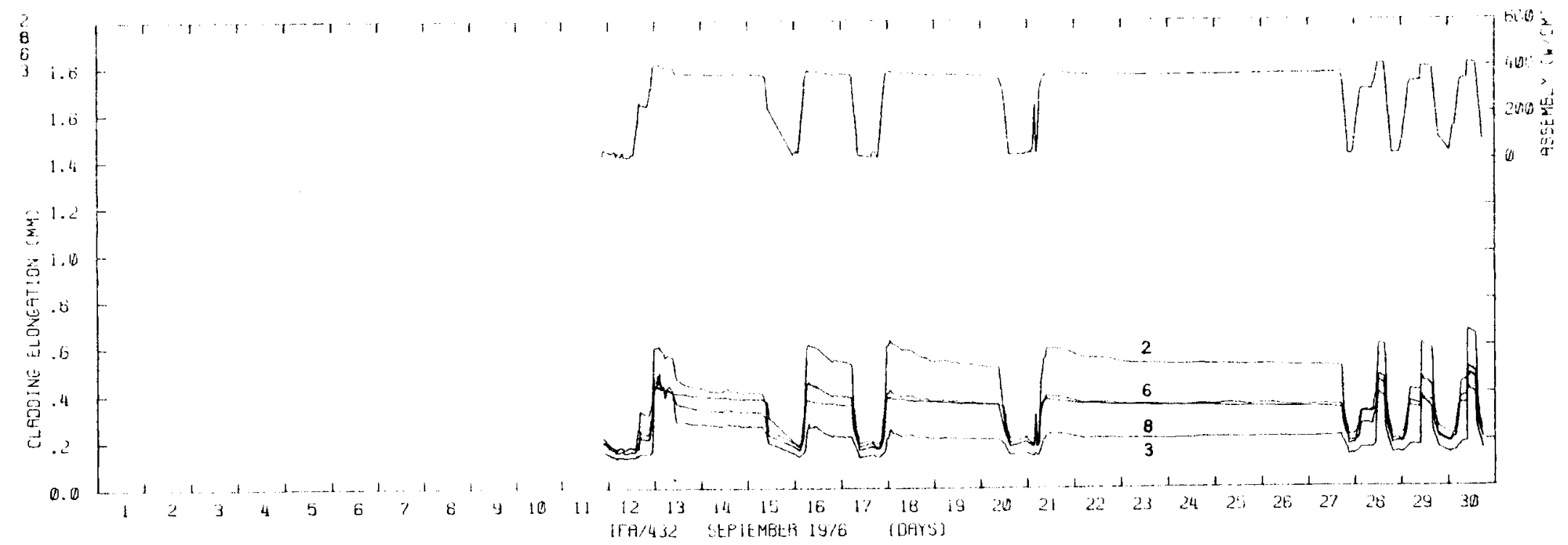

FIGURE 3.196. Cladding Elongation Sensor Readings for Rods 2, 3,6,8 of IFA 432 - September 1976 


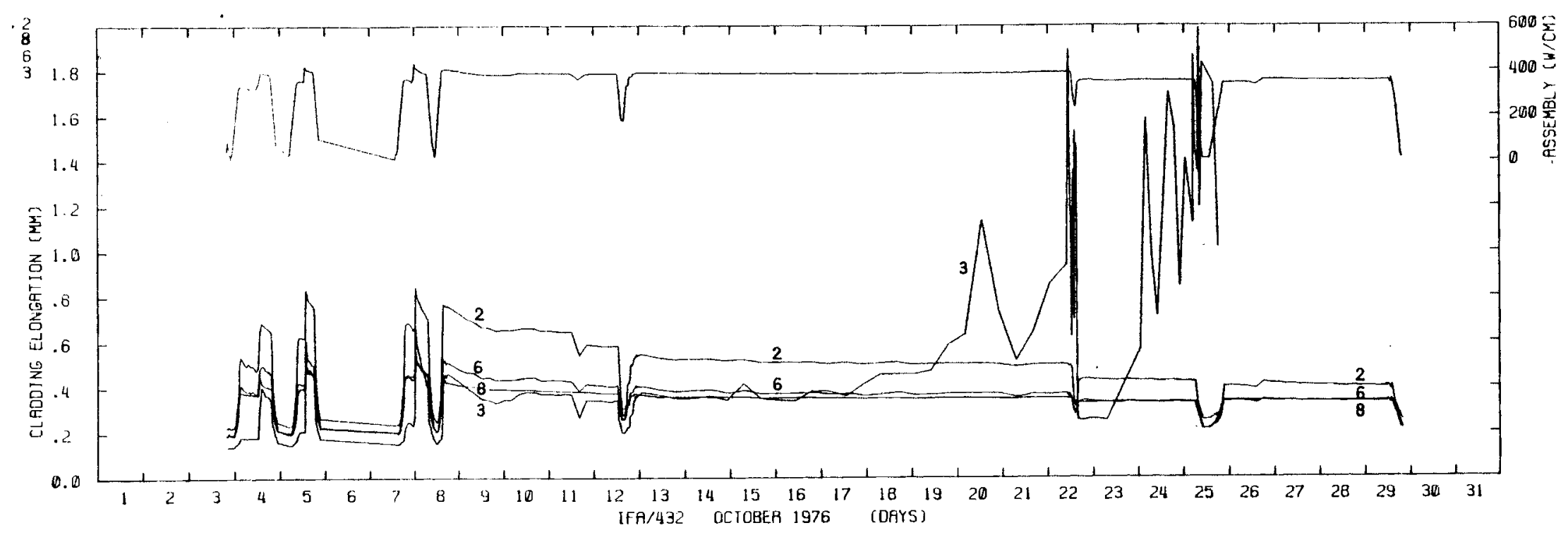

$\stackrel{\omega}{i}$

FIGURE 3.197. Cladding Elongation Sensor Readings for Rods 2, 3, 6, 8 of IFA 432 - October 1976

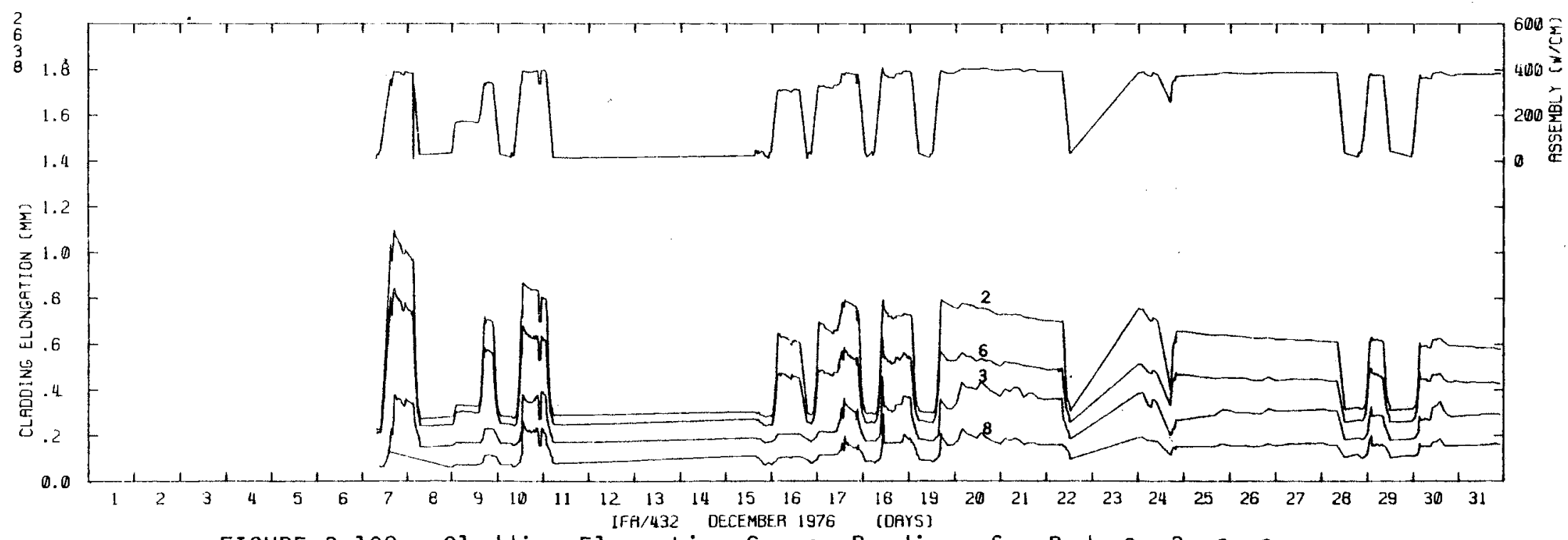

FIGURE 3.198. Cladding Elongation Sensor Readings for Rods 2, 3, 6, 8 of IFA 432 - December 1976 


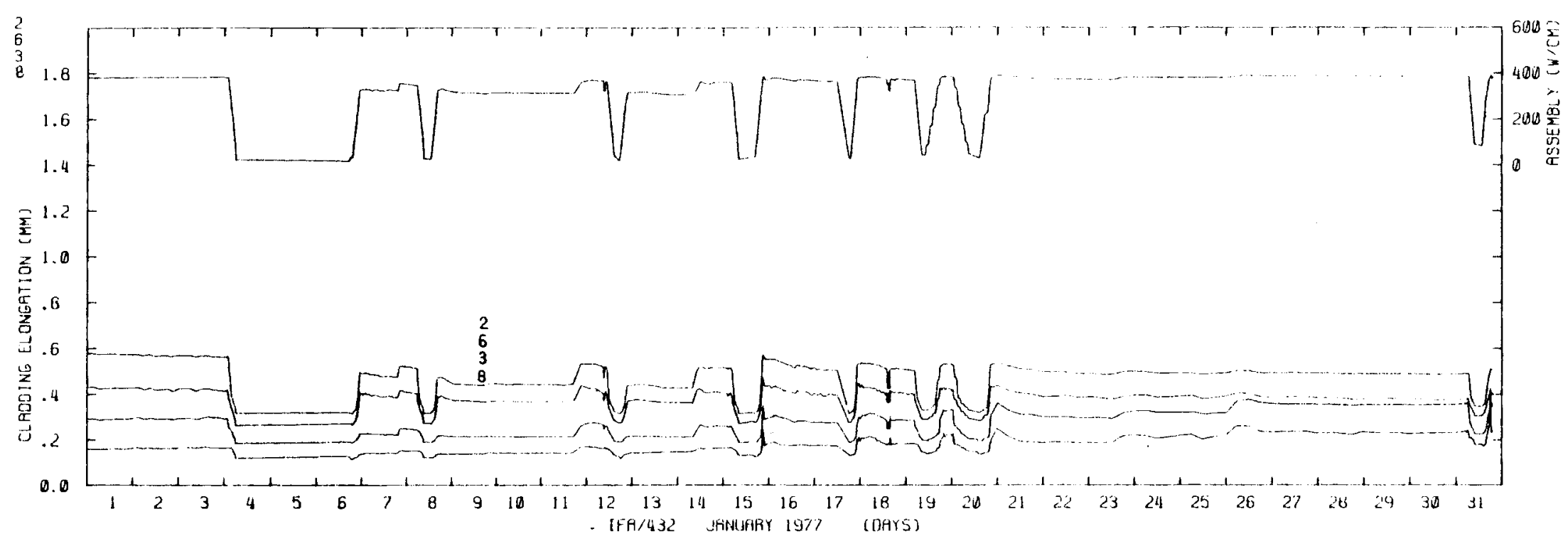

FIGURE 3.199. Cladding Elongation Sensor Readings for Rods 2, 3, 6, 8 of IFA 432 - January 1977

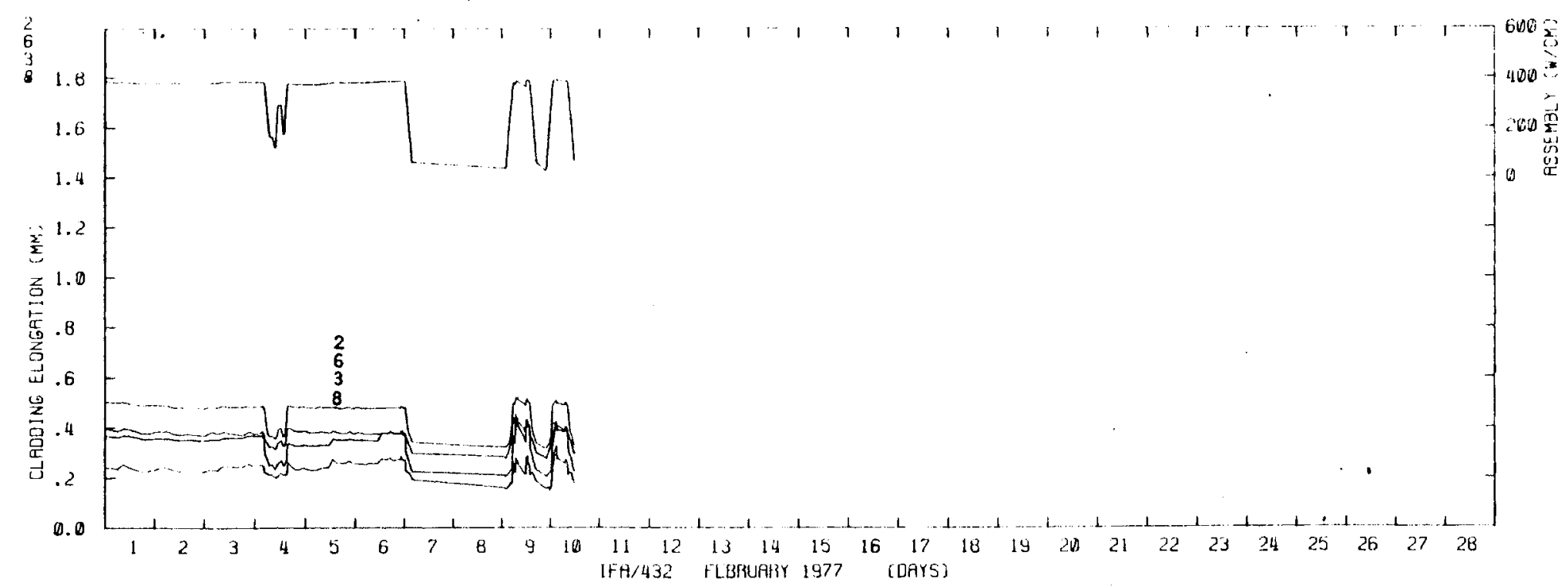

FIGURE 3.200. Cladding Elongation Sensor Readings for Rods 2, 3, 6, 8 of IFA 432 - February 1 ? 


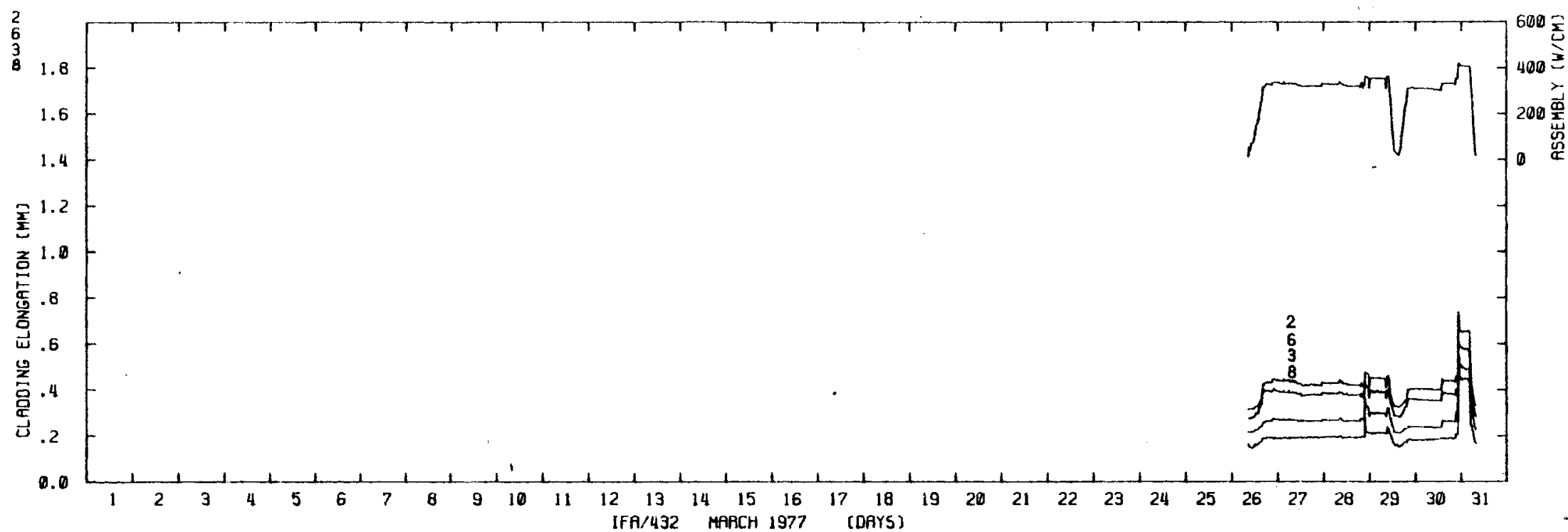

FIGURE 3.201. Cladding Elongation Sensor Readings for Rods 2, 3, 6, 8 of IFA 432 - March 1977

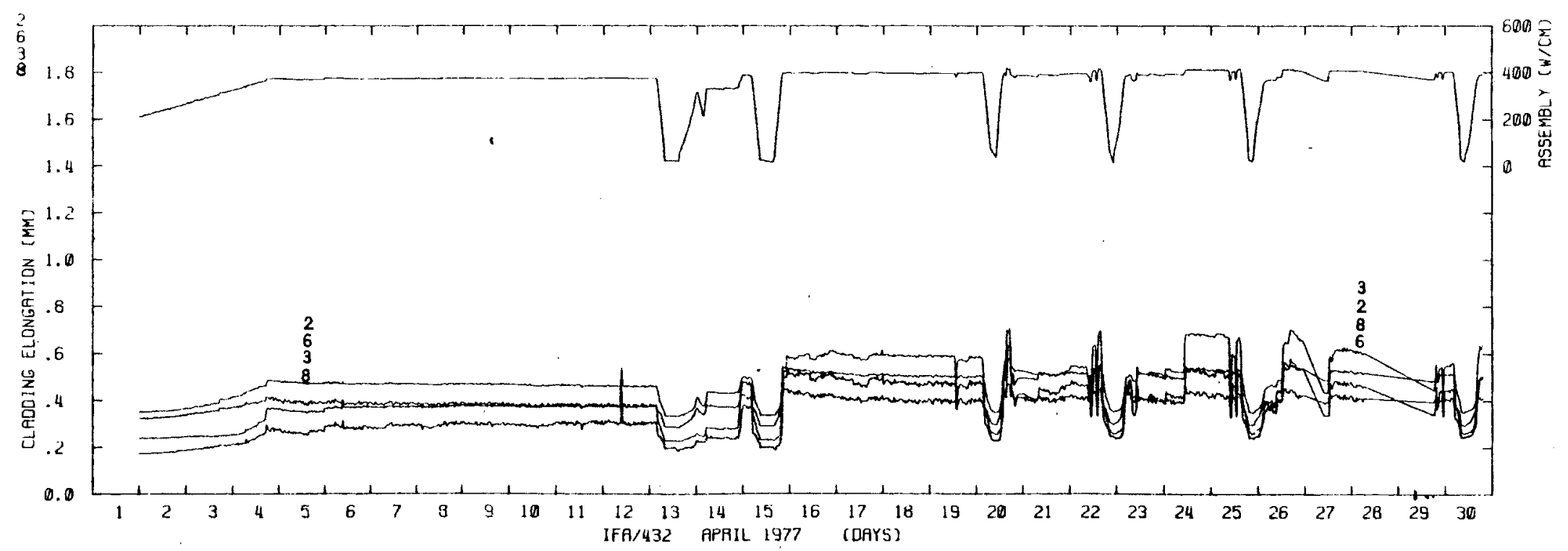

FIGURE 3.202. Cladding Elongation Sensor Readings for Rods 2, 3, 6, 8 of IFA 432 - Apri1 1977 

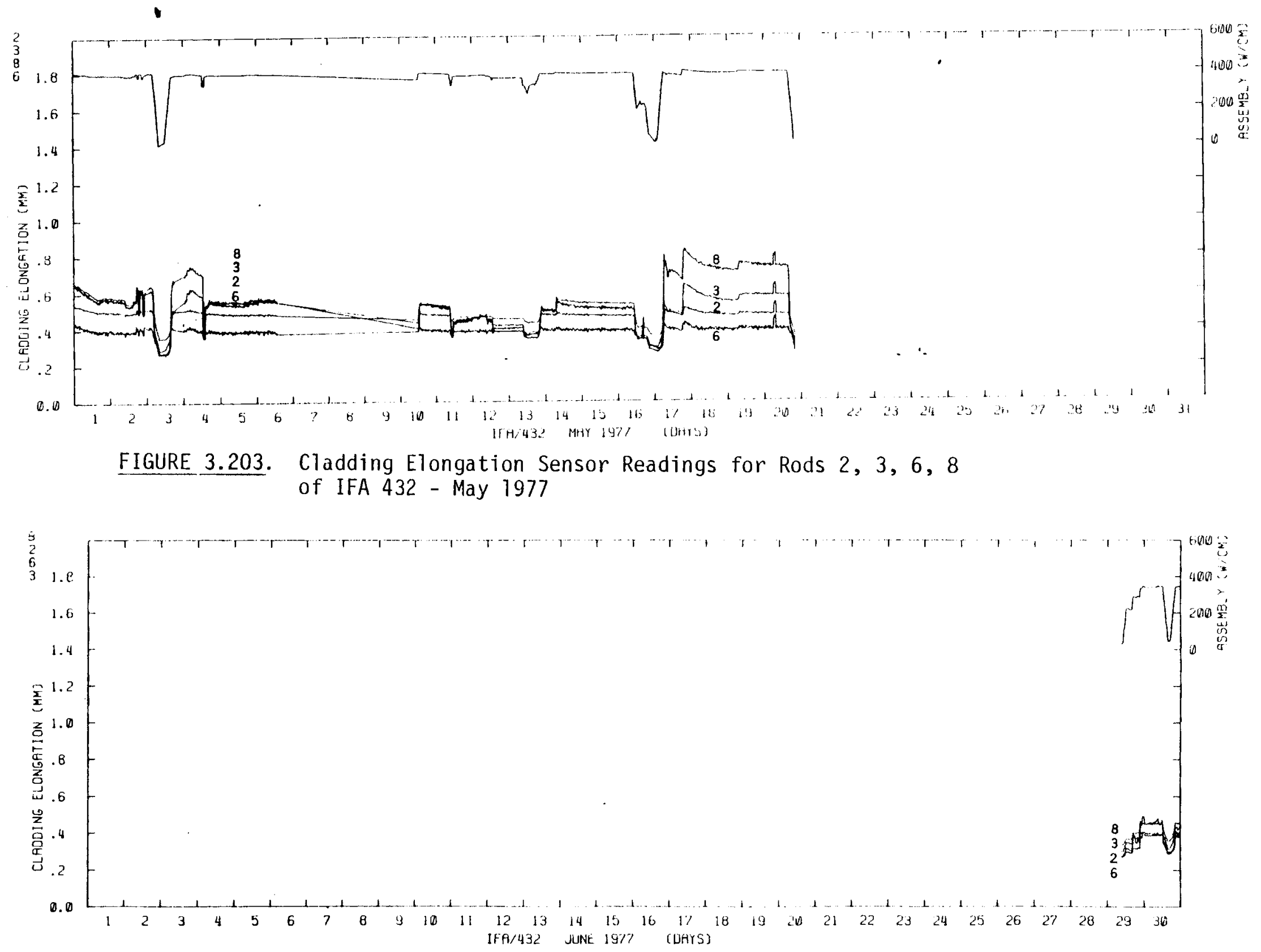

FIGURE 3.204. Cladding Elongation Sensor Readings for Rods 2, 3, 6, 8 gf IFA 432 - June 1977 


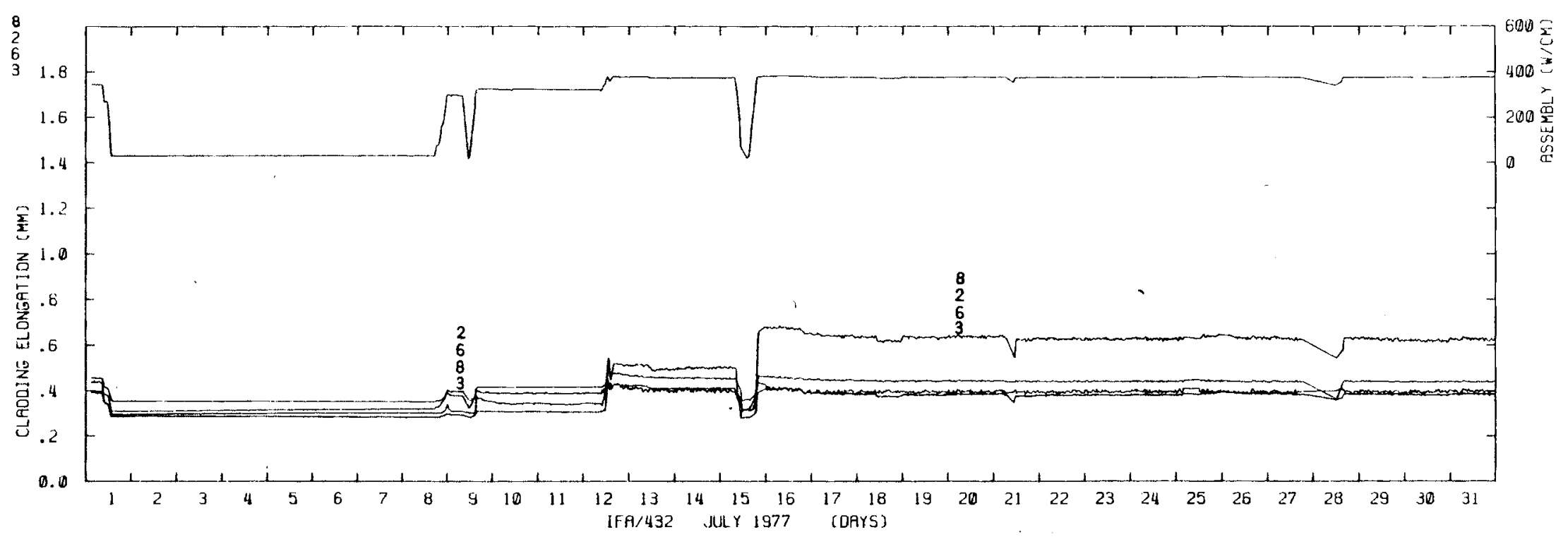

FIGURE 3.205. Cladding Elongation Sensor Readings for Rods 2, 3, 6, 8 of IFA 432 - July 1977

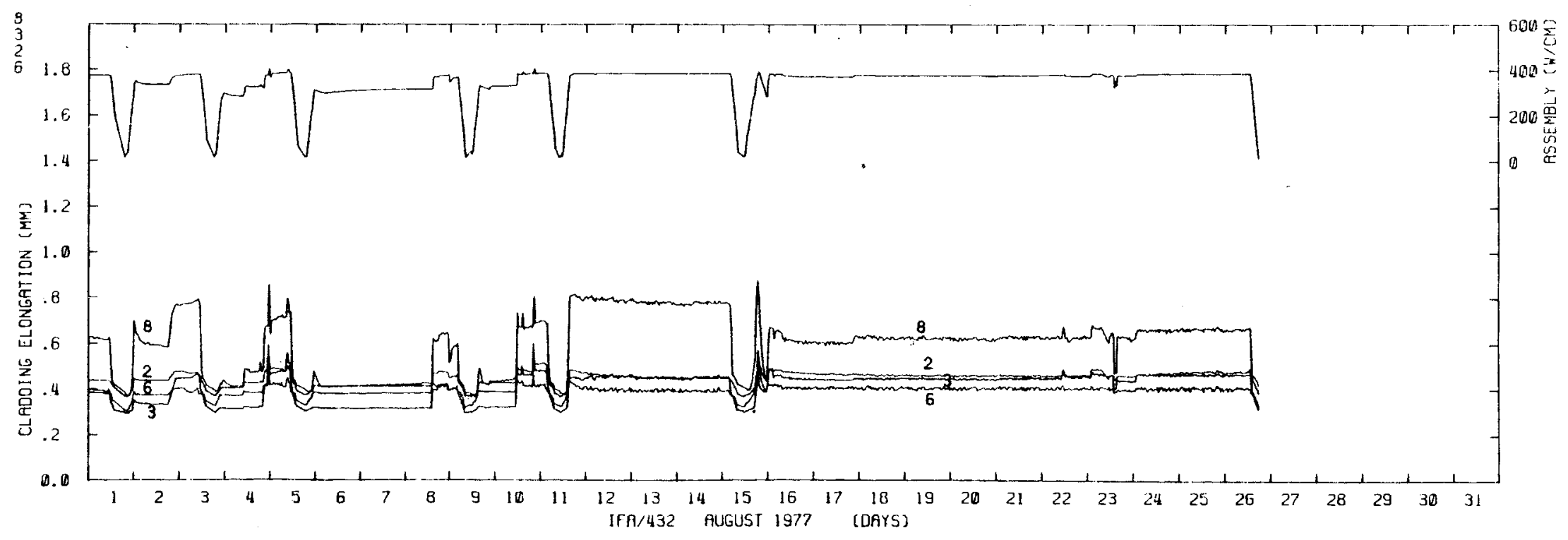

FIGURE 3.206. Cladding Elongation Sensor Readings for Rods 2, 3, 6, 8 of IFA 432 - August 1977 


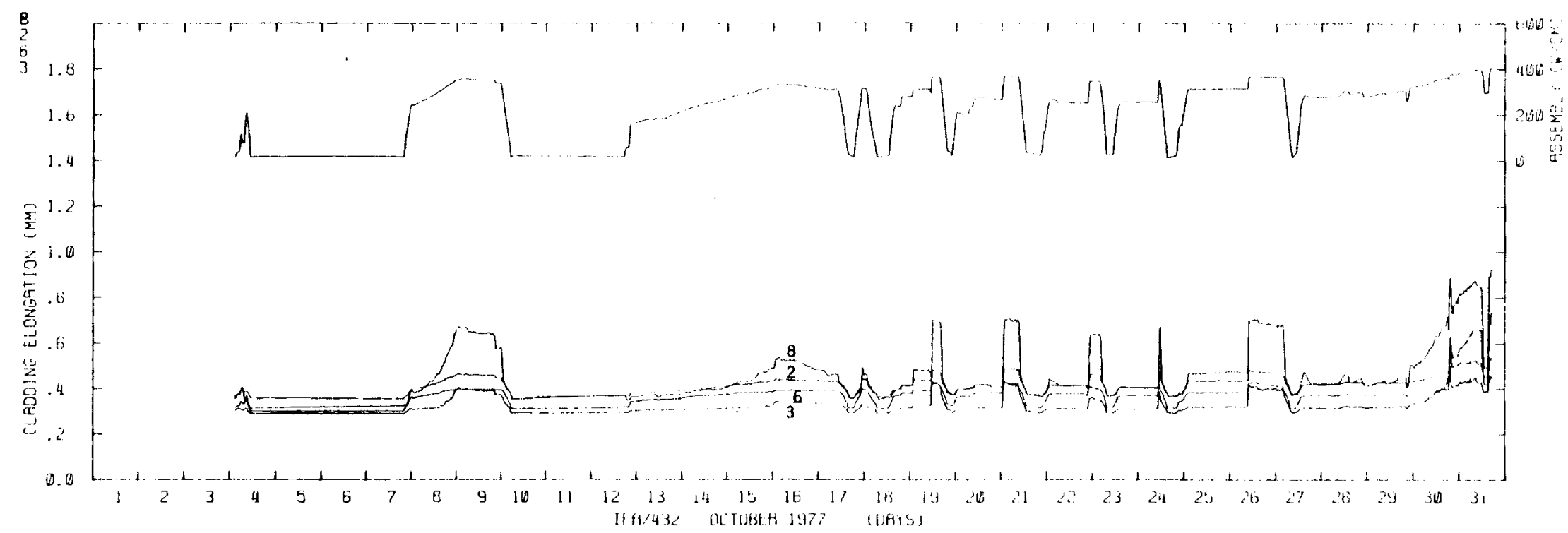

FIGURE 3.207. Cladding Elongation Sensor Readings for Rods 2, 3, 6, 8 of IFA 432 - October 1977

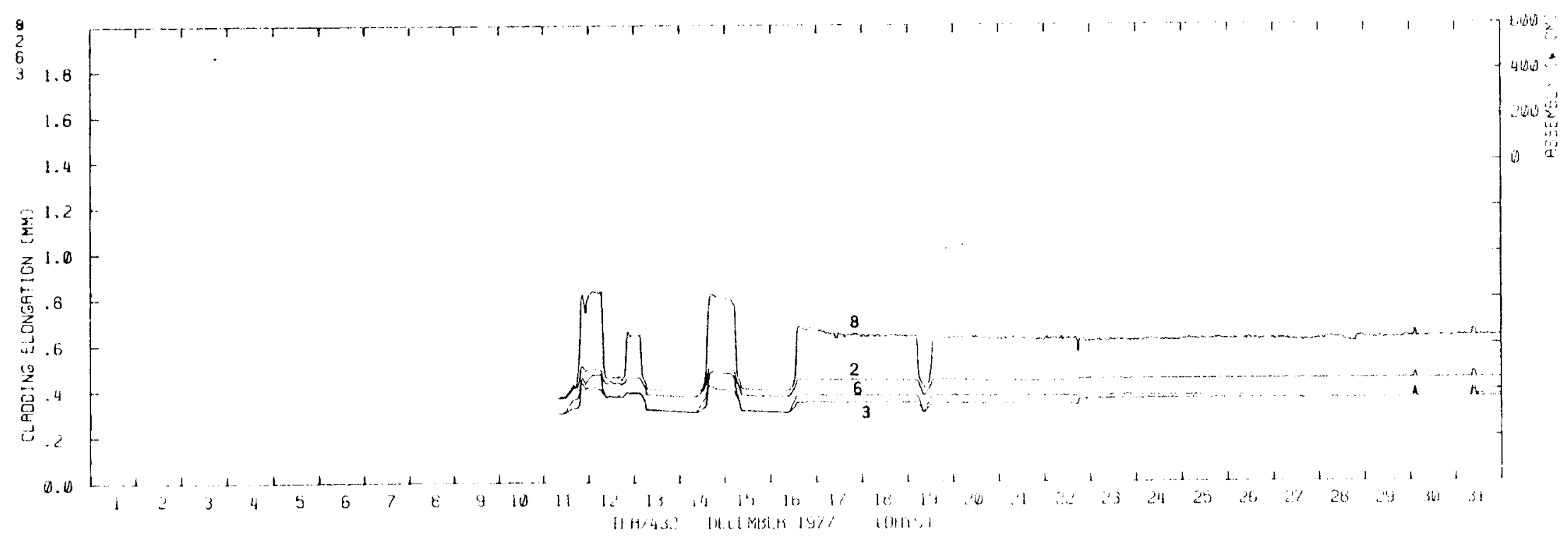

FIGURE 3.208. Cladding Elongation Sensor Readings for Rods 2, 3, 6, 8 of IFA 432 - December 1977 


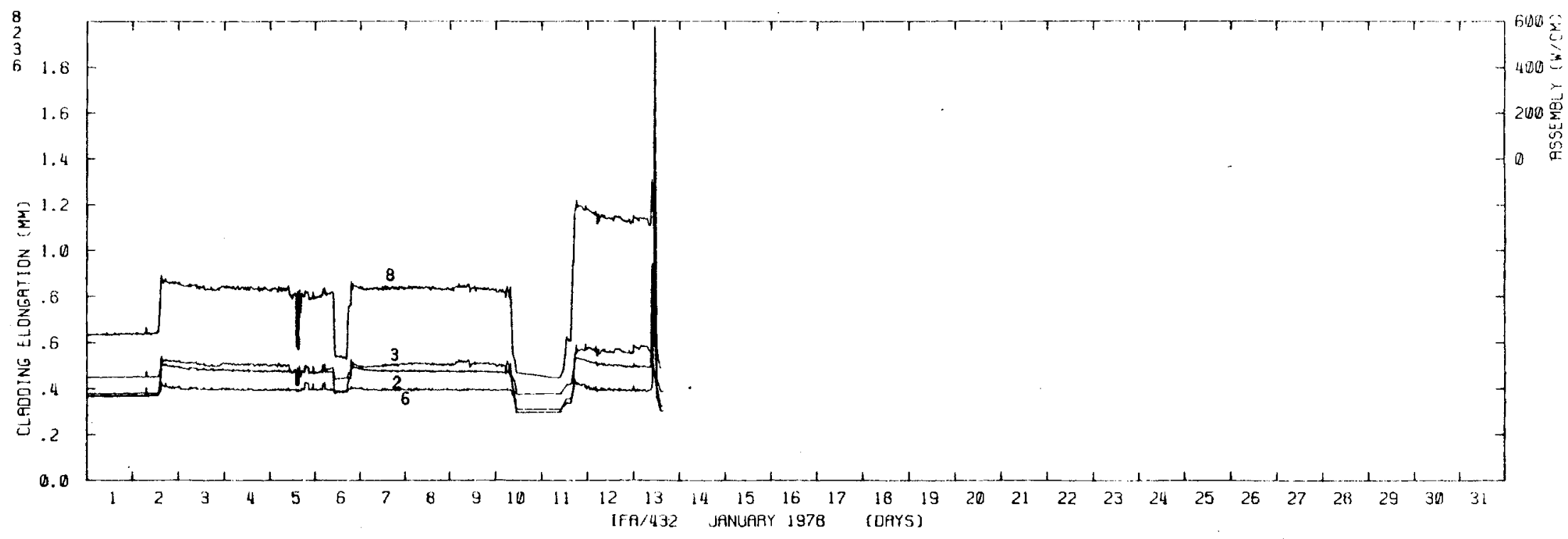

FIGURE 3.209. Cladding Elongation Sensor Readings for Rods 2, 3, 6, 8 of IFA 432 - January 1978

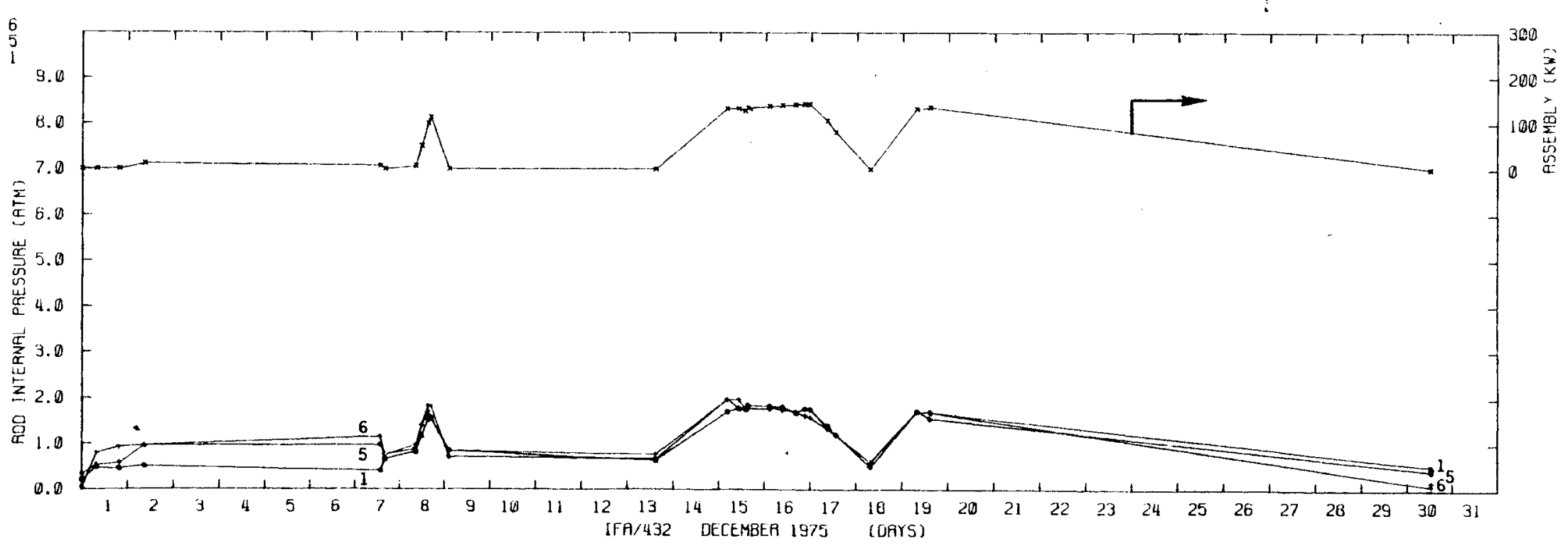

FIGURE 3.210. Internal Pressures for Rods 1,5,6 of IFA 432 - December 1975 

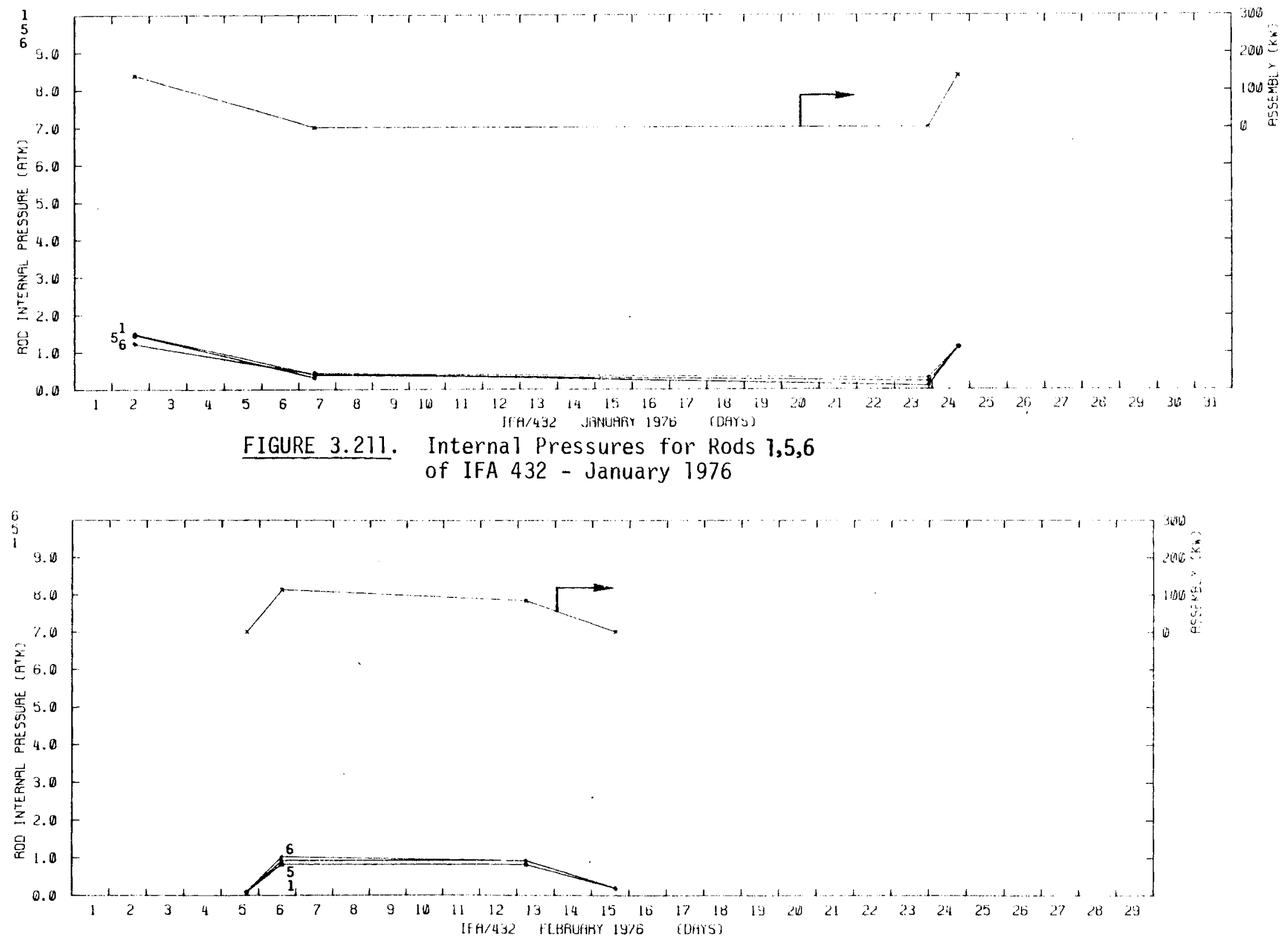

FIGURE 3.212. Internal Pressures for Rods 1, 5, 6 of IFA 432 - February 1976 


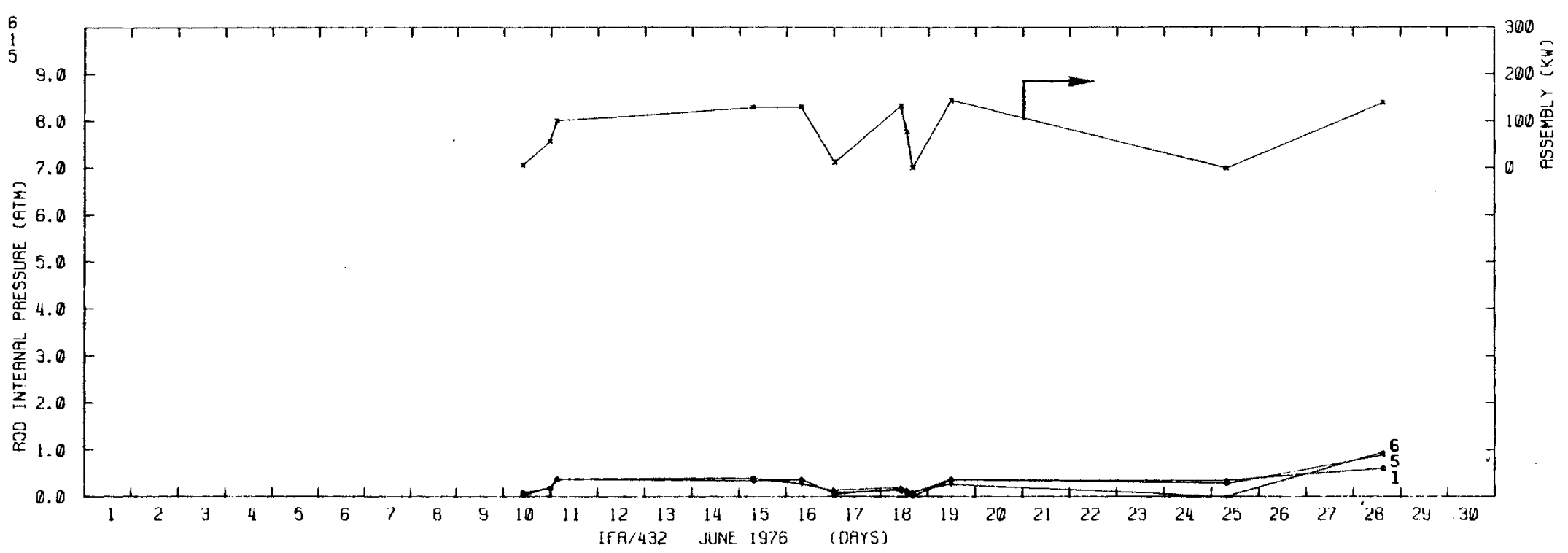

FIGURE 3.213. Internal Pressures for Rods 1, 5, 6 of IFA 432 - June 1976

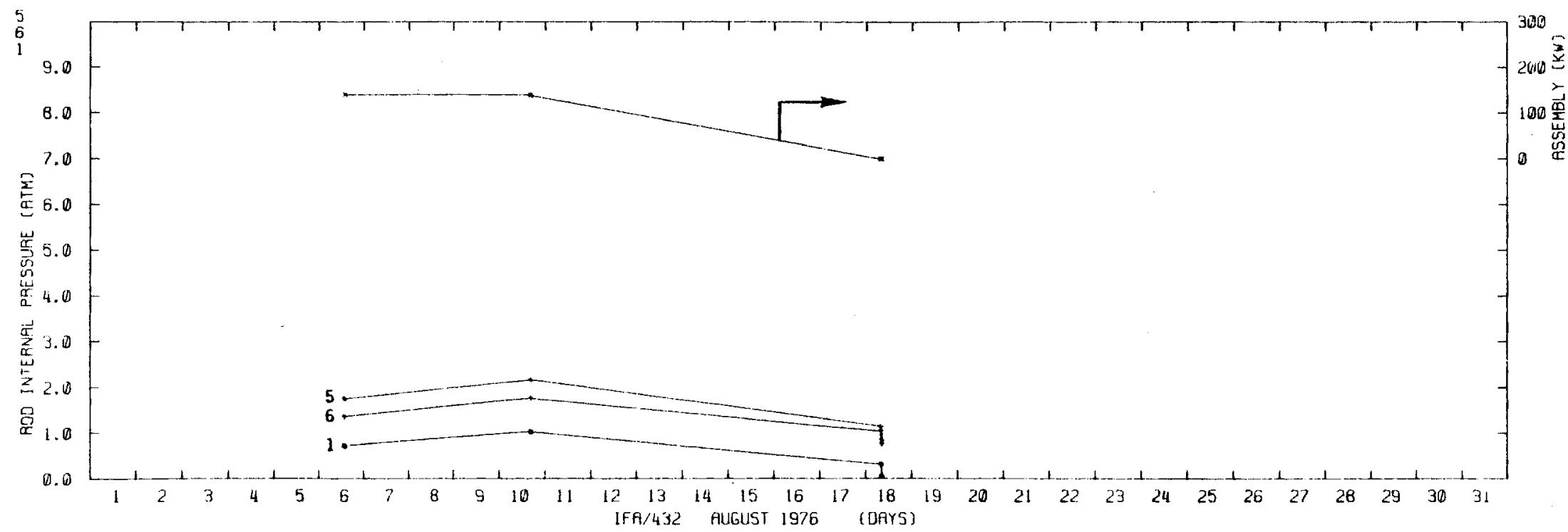

FIGURE 3.214. Internal Pressures for Rods 1, 5, 6 of IFA 432 - August 1976 


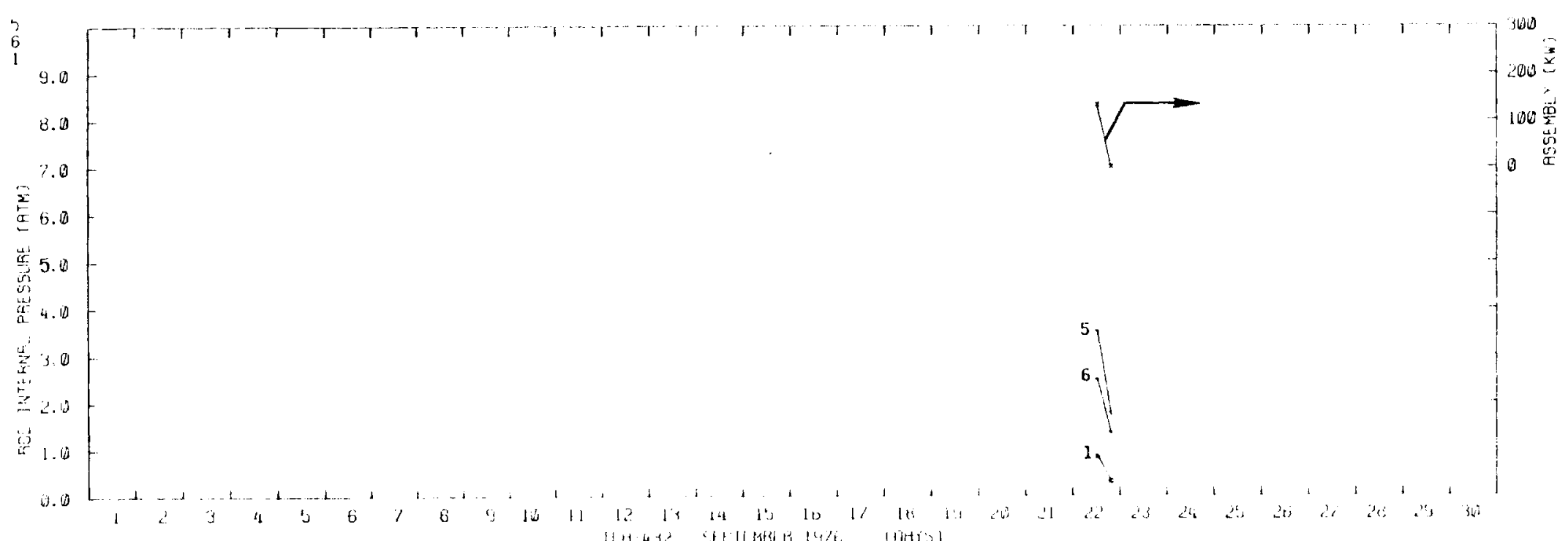

FIGURE 3.215. Internal Pressures for Rods 1, 5, 6 of IFA 432 - September 1976

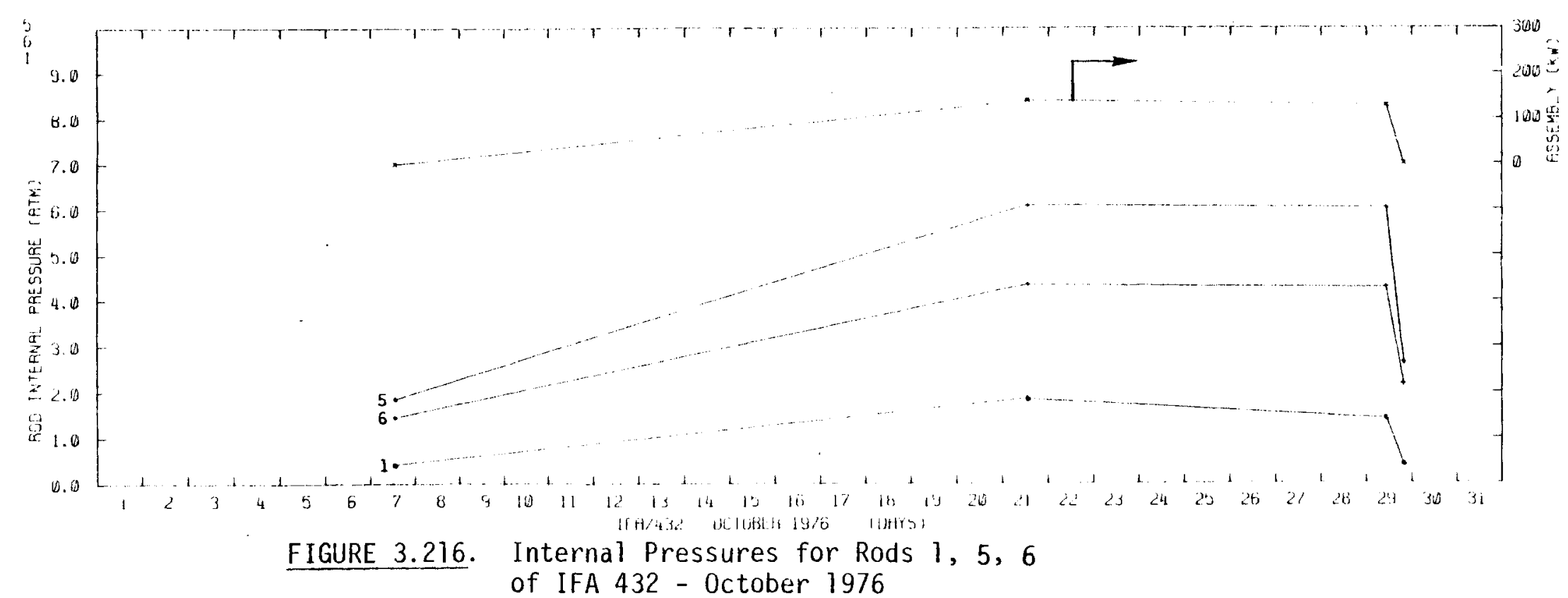




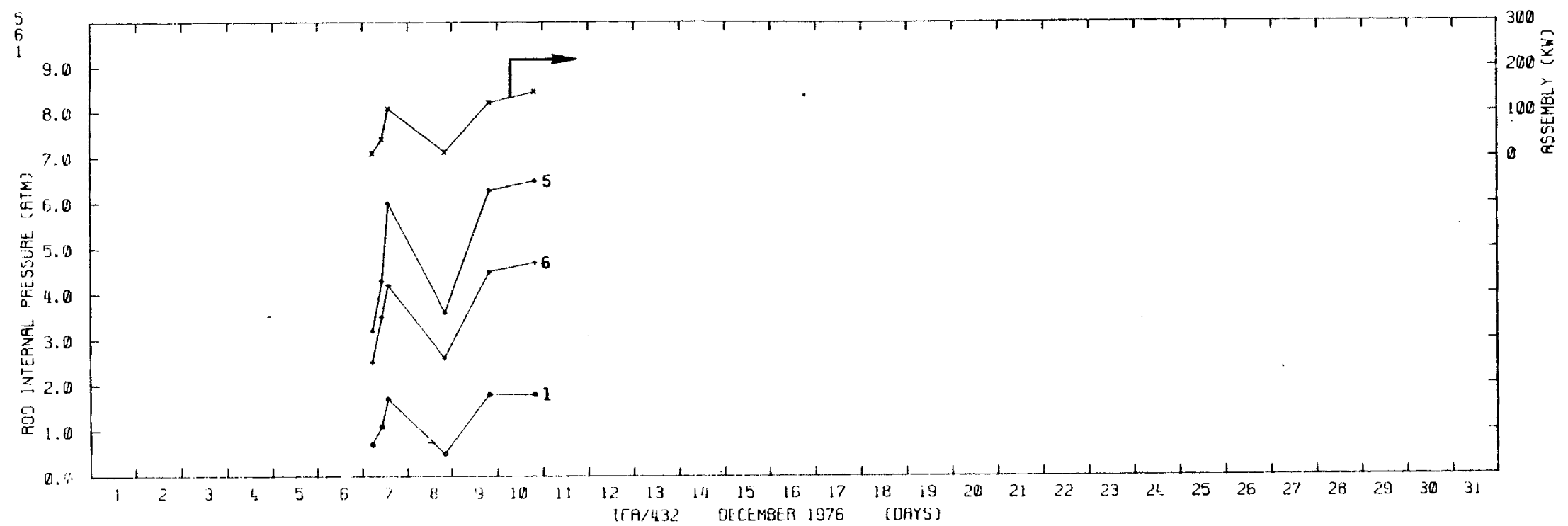

FIGURE 3.217. Internal Pressures for Rods $1,5,6$

of IFA 432 - December 1976

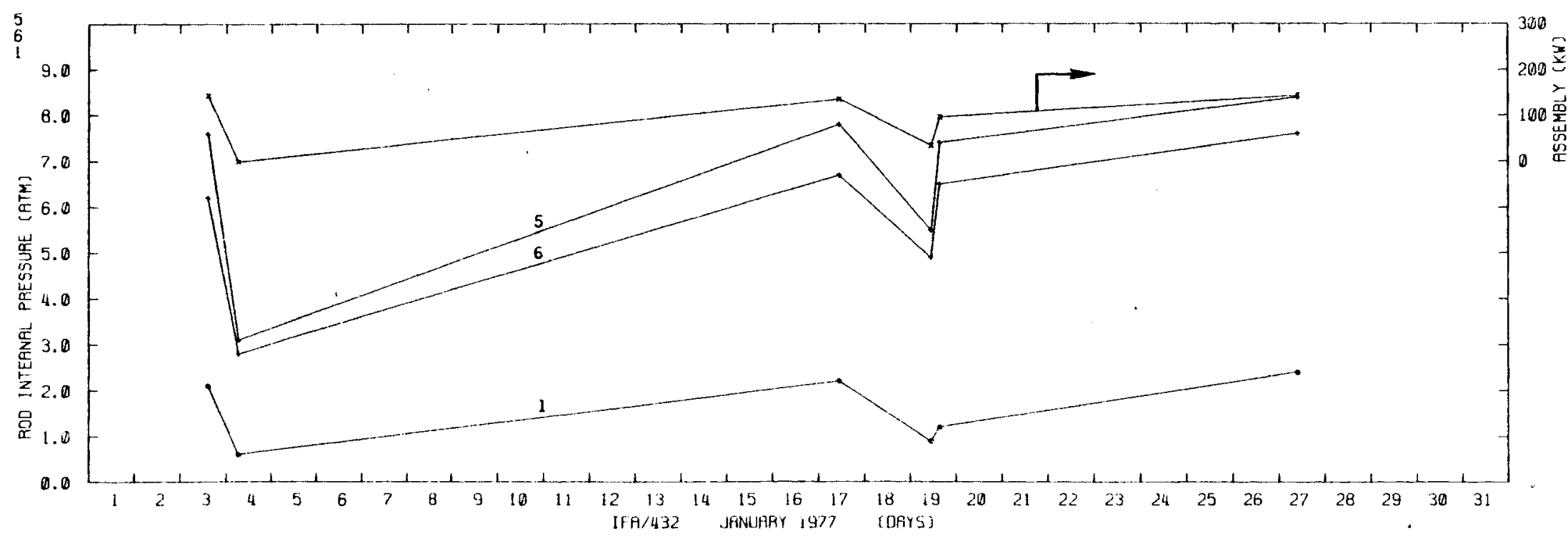

FIGURE 3.218. Internal Pressures for Rods 1, 5, 6 of IFA 432 - January 1977 

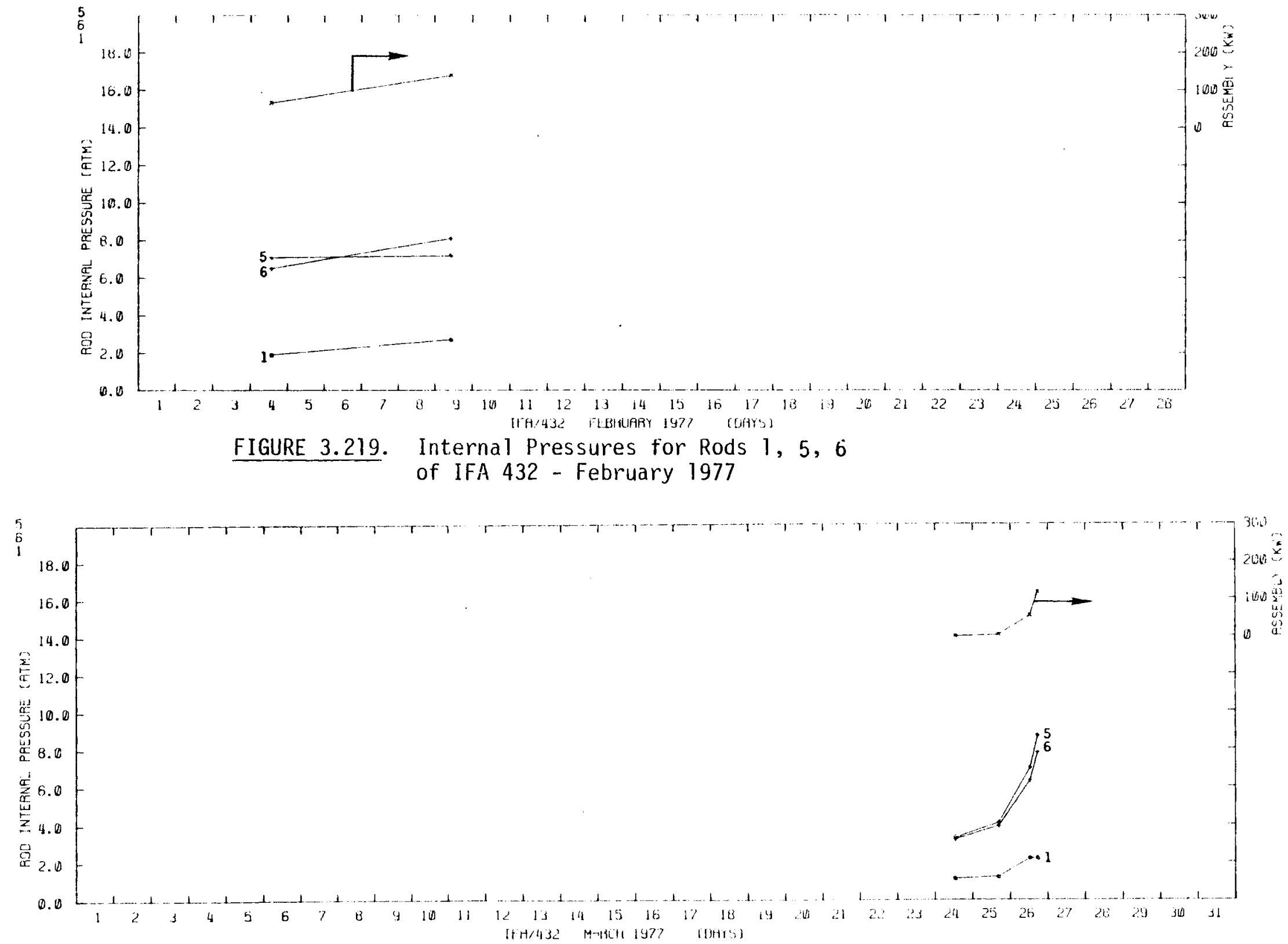

FIGURE 3.220. Internal Pressures for Rods 1, 5, 6 of IFA 432 - March 1977 


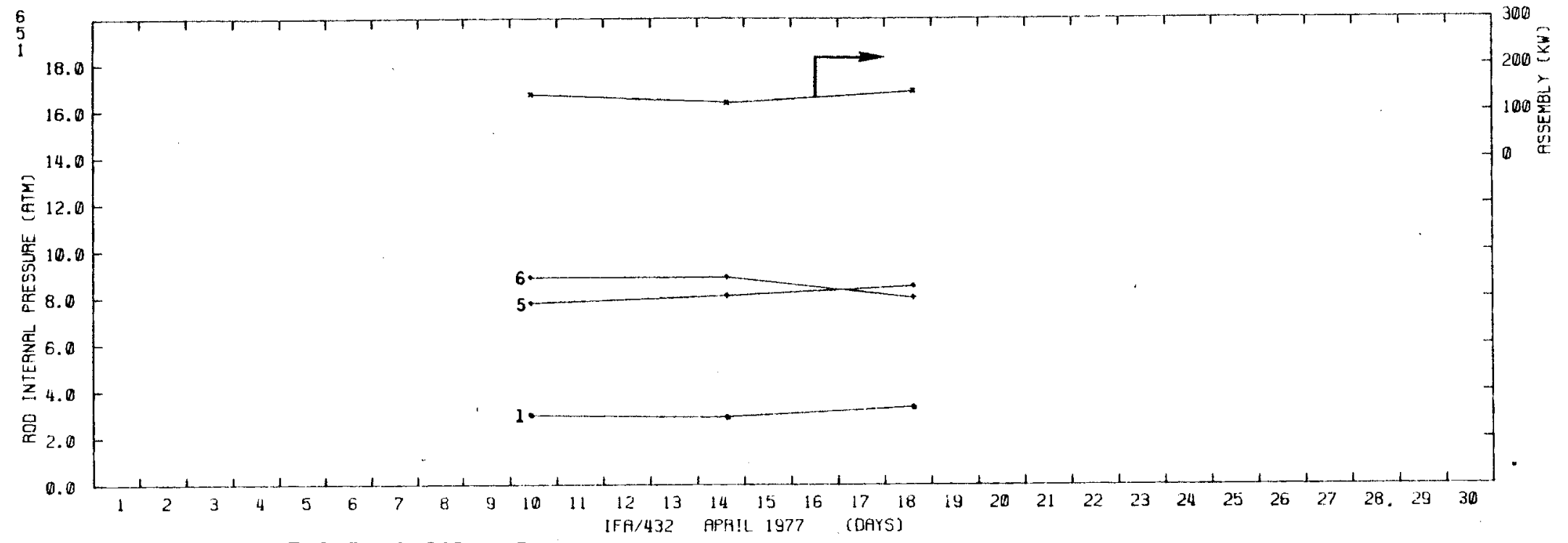

$\stackrel{\omega}{\stackrel{\omega}{\omega}}$

FIGURE 3.221. Internal Pressures for Rods 1, 5, 6

of IFA 432 - Apri1 1977

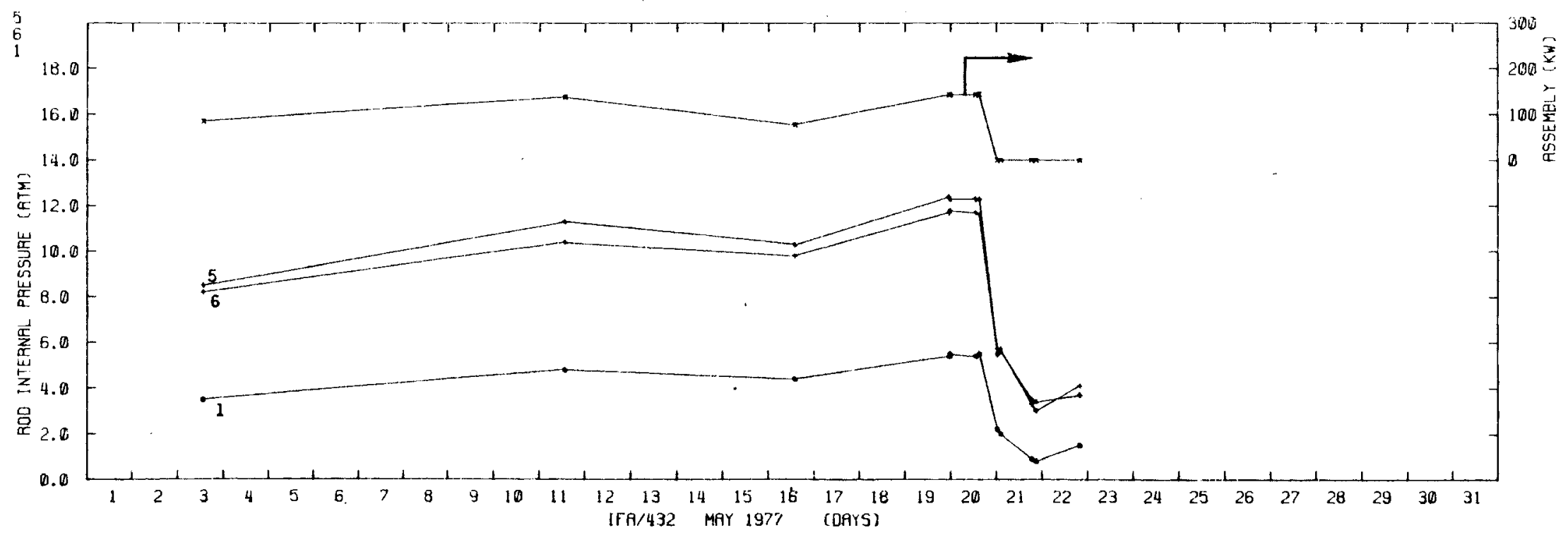

FIGURE 3.222. Internal Pressures for Rods 1, 5, 6 of IFA 432 - May 1977 


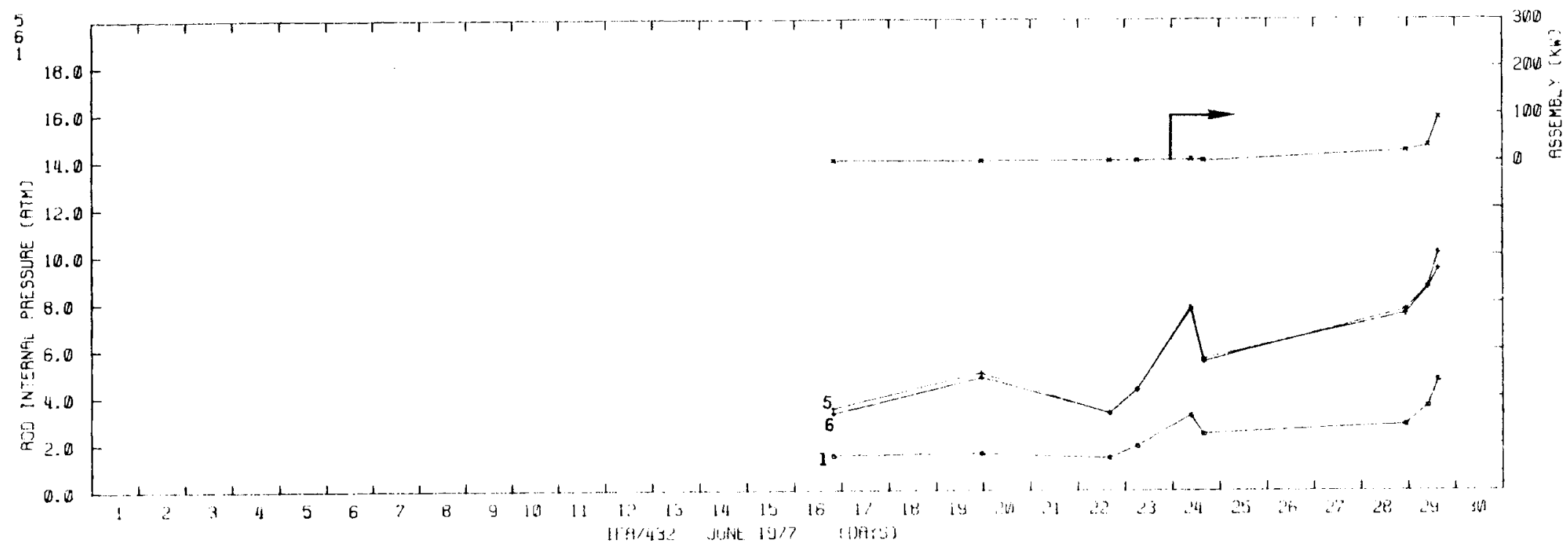

FIGURE 3.223. Internal Pressures for Rods 1, 5, 6 of IFA 432 - June 1977

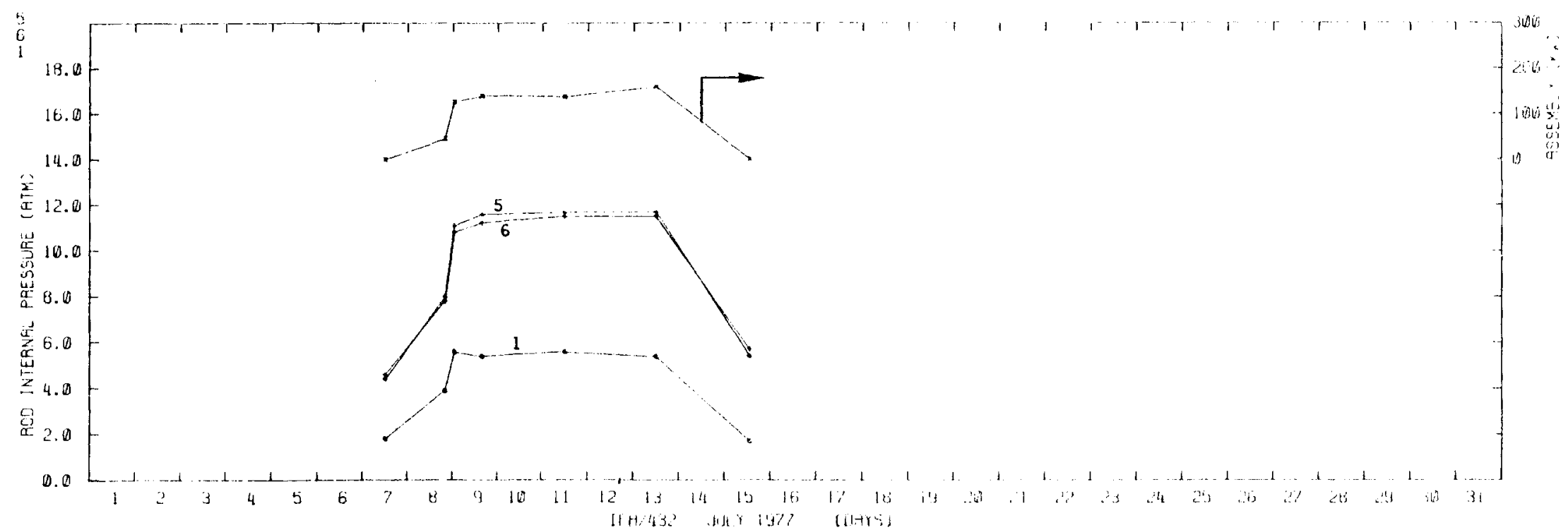

FIGURE 3.224. Internal Pressures for Rods 1, 5, 6 of IFA 432 - July 1977 


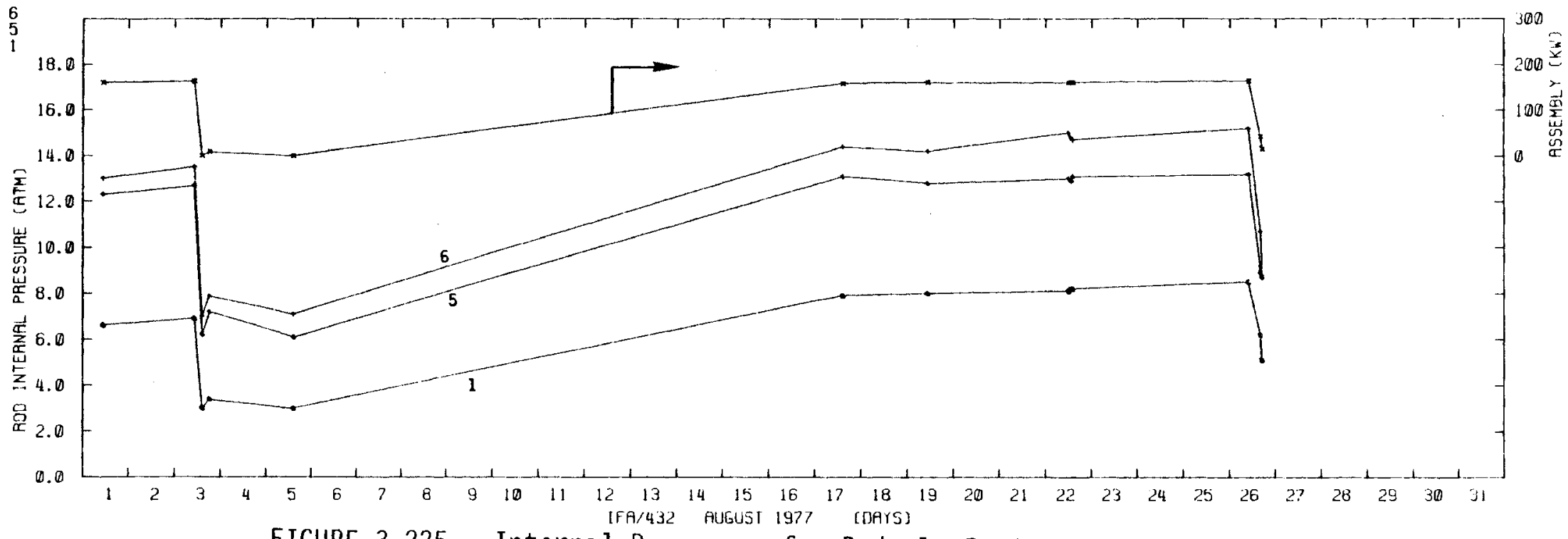

FIGURE 3.225. Internal Pressures for Rods 1, 5, 6

of IFA 432 - August 1977

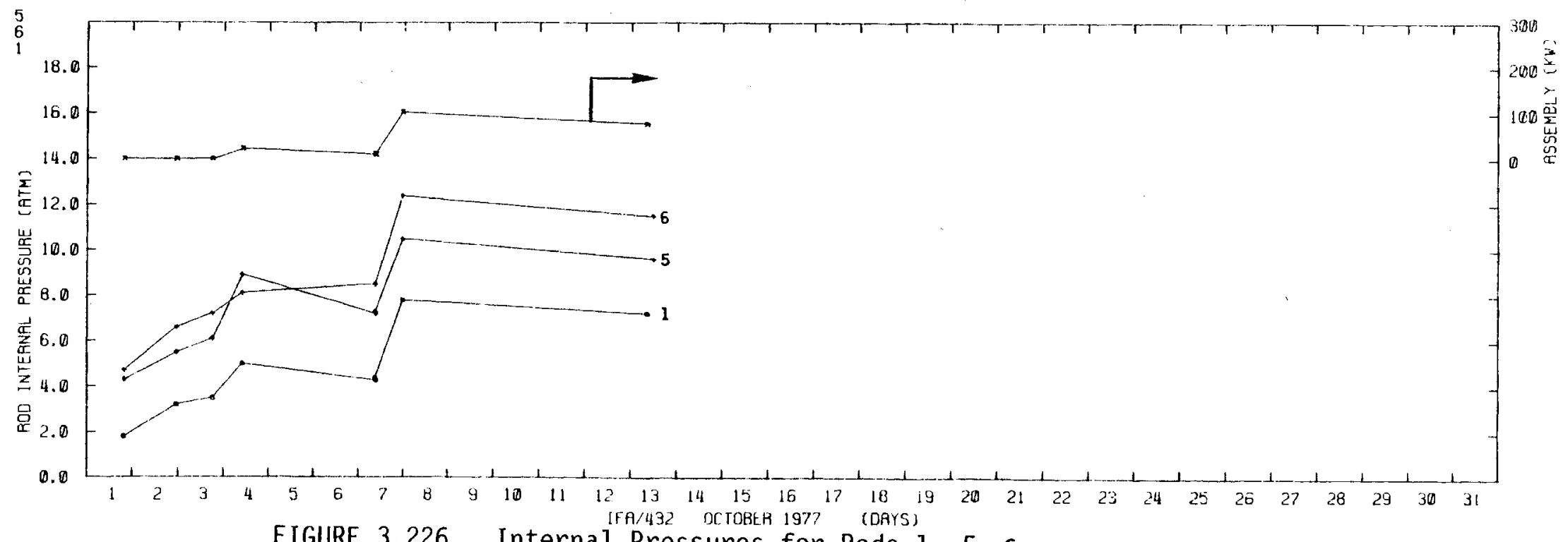

FIGURE 3.226. Internal Pressures for Rods $1,5,6$ of IFA 432 - October 1977 
TABLE 3.1. IFA-432 Burnup (MWD/MTM).

\begin{tabular}{|c|c|c|c|c|c|c|c|c|c|c|c|c|c|}
\hline \multirow[b]{2}{*}{ Manth } & \multirow[b]{2}{*}{ Year } & \multicolumn{6}{|c|}{ Upper TC } & \multicolumn{6}{|c|}{ Lower TC } \\
\hline & & Rod 1 & Rod 2 & Rod 3 & Rod 4 & Rod 5 & R.oó 6 & Rod 1 & Roo 2 & Rod 3 & $\operatorname{Rod} 4$ & Rod 5 & Rod 5 \\
\hline 12 & 75 & 813.2 & 793.4 & 837.4 & 864.5 & 383.4 & 846.6 & 596.0 & 596.5 & 623.5 & 630.9 & 637.7 & 616.0 \\
\hline 1 & 76 & 2210.2 & 2753.8 & 2254.4 & 2312.9 & 2366.4 & 2285.2 & 1609.3 & 1611.2 & $: 687.7$ & 1710.7 & 1728.4 & 1666.4 \\
\hline 2 & 76 & 2746.7 & 2675.2 & 2796.2 & 2866.8 & 2934.7 & 2837.6 & 1989.4 & 1991.9 & 2088.1 & 2118.3 & 2140.0 & 2061.6 \\
\hline 6 & 76 & 3510.7 & 3418.6 & 3564.1 & 784.1 & 3733.6 & 3618.7 & 2507.3 & 2512.0 & 2632.7 & 549.6 & 2693.3 & 2595.6 \\
\hline 7 & 76 & 4978.1 & 4843.4 & 5030.9 & 2281.2 & 5261.9 & 5117.5 & 3529.8 & 3536.6 & 3696.7 & 1621.1 & 3773.8 & 3645.2 \\
\hline 8 & 76 & 5853.8 & 5694.6 & 5907.2 & $3174 . j$ & 6172.9 & 6011.0 & 4121.8 & 4131.1 & 4317.6 & 2247.7 & 4404.3 & 4254.6 \\
\hline 9 & 76 & 6627.5 & 6448.9 & 6680.8 & 3959.0 & 6970.6 & 6795.9 & 4656.9 & 4667.3 & 4871.9 & 2803.3 & 4964.4 & 4801.2 \\
\hline 10 & 76 & 7805.2 & 7597.7 & 7856.8 & 5148.8 & 8179.6 & 7987.7 & 5489.9 & 5500.4 & $5: 35.2$ & 3671.9 & 5841.6 & 5655.6 \\
\hline 12 & 76 & 8485.3 & 8241.1 & 8502.0 & 5808.9 & 8870.7 & 8682.5 & 6001.7 & 5996.1 & 6234.3 & 4176.3 & 6367.1 & 6181.9 \\
\hline 1 & 77 & 9757.8 & 9449.6 & 9716.1 & 7048.8 & i0163.7 & 9980.2 & 6958.6 & 6924.4 & 7163.9 & 5109.3 & 7337.7 & 7159.2 \\
\hline 2 & $\pi$ & 10130.6 & 9801.5 & 10068.0 & 7409.2 & 10541.8 & 10361.2 & 7231.5 & 7189.0 & 7429.0 & 5375.7 & 7614.9 & 7438.1 \\
\hline 3 & $\pi$ & 10313.2 & 9978.5 & 10249.0 & 7593.3 & 70730.0 & 10546.9 & 7376.8 & 7330.3 & 7574.2 & 5524.7 & 7769.4 & 7589.9 \\
\hline 4 & 7 & 11528.5 & 11139.4 & 11423.8 & 8795.2 & 11975.8 & 11788.6 & 8305.7 & 8229.9 & 8495.7 & 6471.3 & 8754.4 & 8560.8 \\
\hline 5 & 77 & 12334.2 & 11908.8 & 12201.5 & 9590.5 & 12800.5 & 12611.4 & 8914.5 & 8819.6 & 9100.1 & 7092.6 & 9400.4 & 9197.2 \\
\hline 6 & 77 & 12389.3 & 11961.7 & 12254.5 & 9644.0 & 12855.6 & 12666.9 & 8958.6 & 8363.4 & 9144.8 & 7137.2 & 9445.9 & 9242.2 \\
\hline 7 & 77 & 13560.6 & 13031.4 & 13273.2 & 10676.8 & 13979.1 & 13849.9 & 9760.2 & 9616.4 & 9875.1 & 7873.0 & 10236.8 & 10062.2 \\
\hline 8 & $\pi$ & 14762.8 & 14134.1 & 14325.9 & 11742.0 & 15132.5 & 15061.9 & 70578.8 & 10387.4 & 10622.0 & 8623.1 & 11041.2 & 10896.9 \\
\hline 10 & 77 & 15556.2 & 14881.8 & 15056.5 & 12476.3 & 15905.3 & 15857.7 & 11160.8 & 10945.0 & 11185.7 & 9201.7 & 11649.4 & 11503.7 \\
\hline 11 & 77 & 15677.4 & 14995.3 & 15156.0 & 12585.8 & 16021.3 & 15978.5 & 11246.5 & 11027.5 & 11268.8 & 9286.2 & 11737.7 & 11592.2 \\
\hline 12 & 7 & 16393.6 & 15693.5 & 15865.8 & 13282.4 & 16729.4 & 16690.6 & 11770.4 & 11542.2 & 11796.9 & 9822.8 & 12288.3 & 12133.7 \\
\hline 1 & 72 & 15931.6 & 16207.4 & 16373.5 & 13791.4 & 17257.5 & 17228.9 & 12150.4 & 11913.0 & 12175.6 & 10208.3 & 12686.3 & 12527.0 \\
\hline
\end{tabular}




\section{REFERENCES}

1. D. W. Brite, J. L. Daniel, N. C. Davis, M. D. Freshley, P. E. Hart, and R. K. Marsha11, EEI/EPRI Fuel Densification Project Research Project 131 Final Report prepared for Electric Power Research Instititue by Battelle, Pacific Northwest Laboratories, March 1975, Revised June 1975.

2. Regulatory Staff, U.S. Atomic Energy Commission, Technical Report on Densification of Light Water Reactor Fuels, WASH-1236, November 1972.

3. M. D. Freshley, P. E. Hart, J. L. Daniel, D. W. Brite, and T. D. Chikalla, "The Effect of Pellet Characteristics and Irradiation Conditions on $\mathrm{UO}_{2}$ Fuel Densification." Presented at the ANS/CNA Topical Meeting on "Commercial Nuclear Fuel - Current Technology," Toronto, Canada, April 1975.

4. A. Hanevik, K. D. Knudsen, and P. Arnesen, "In-Reactor Measurements of Fuel Stack Shortening." Proceedings of the British Nuclear Energy Society Conference, London, England, October 1973.

5. J. A. L. Robertson, SkDe in Fuel Irradiations. CRFD-835, Chalk River, Ontario, Canada, Apri1 1959.

6. D. D. Lanning and C. R. Hann, Verification of Fuel Centerline Thermocouple Readings Through Response to Linear Power Decreases, BNWL-2189, Battelle, Pacific Northwest Laboratories, Richland, WA 99352, April 1977.

7. I. Cohen, B. Lustman and J. D. Eichenberg, Measurement of the Therma 1 Conductivity of Metal-Clad Uranium Oxide Rods During Irradiation. WAPD-228, Westinghouse Electric Corporation, Bettis Atomic Power Laboratory, Pittsburgh, PA, August 1960.

8. G. R.Horn, "The Effects of Xenon on Fuel-to-Cladding Gap Conductivity." Trans. ANS, Vol. 12, p. 607, 1969.

9. L. A. Lawrence, G. R. Horn and J. A. Christensen, "Fuel-Clad Gap Conductions in Fast Reactor Fuels." Trans. ANS. Vol. 13, p. 572, 1970.

10. D. J. Bradley and C. R. Hann, An Evaluation of the Discrepancies Between Predicted and Experimental Effects of Xenon and Krypton in Nuclear Fuel Rods. BNWL-1955, Battelle, Pacific Northwest Laboratories, Richland, WA 99352, November 1975 .

11. R. E. Williford and C. R. Hann, Effects of Fill Gas Composition and Pellet Eccentricity, BNWL-2285, Battelle, Pacific Northwest Laboratories, Richland, WA 99352, July 1977.

12. P. E. Hart and J. L. Daniel, "The Role of Microstructure in Thermal and Irradiation-Induced Densification of $\mathrm{NO}_{2}$ Fuels." Presented at 77 th Annual Meeting of the American Ceramic Society, Washington, D.C., May 3-8, 1975. 
13. Core Performance Branch, U.S. Nuclear Regulatory Commission, The Analysis of Fuel Densification, NUREG-0085, July 1976.

14. "An Acceptable Model and Related Statistical Methods for the Analysis of Fuel Densification," Regulatory Guide 1.126, U.S. Nuclear Regulatory Commission, March 1977.

15. C. R. Hann, J. L. Bates, D. W. Brite, J. L. Daniel, N. C. Davis, P. E. Hart, R. K. Marshall, G. Mellinger, and R. E. Williford, Test Design, PreCharacterization and Fuel Assembly Fabrication for Instrumented Fuel Assemblies IFA-437 and IFA-432. BNWL-1988, Battelle, Pacific Northwest Laboratories, Richland, WA 99352 , November 1977.

16. M. F. Lyons, D. H. Coplin, T. J. Pashos, and B. Weidenbaum, U02 Pellet Thermal Conductivity from Irradiation with Central Melting, GEAP-4624, 1964.

17. C. E. Beyer, C. R. Hann, D. D. Lanning, F. E. Panisko and L. J. Parchen, GAPCON-THERMAL-2: A Computer Program for Calculating the Thermal Behavior of an Oxide Fuel Rod. BNWL-1898, Battelle, Pacific Northwest Laboratories, Richland, WA 99352, November 1975.

18. C. R. Hann and R. K. Marshall, Comparative Analys is of Pellet-Cladding Interaction from IFA-431 and IFA-432 Halden Reactor Tests, BNWL-2240, Battelle, Pacific Northwest Laboratories, Richland, WA 99352, July 1977.

19. C. R. Hann, D. D. Lanning, R. K. Marsha11, A. R. Olsen, and R. E. Williford, A Metiod for Determining the Uncertainty of Gap Conductance Deduced from Measured Fuel Centerline Temperatures, BNWL-2091, Battelle, Pacific Northwest Laboratories, Richland, WA 99352, February 1977.

20. Magnetic Properties; Metallic Materials for Thermostats, Electrical Resistance, Heating, Contacts; Temperature Measurements; I11uminating Standards. 01-044077-40, 1977 Annual Book of ASTM Standards (part 44), Proposed Standard, pg. 867-873.

21. L. C. Lynnworth, et. a1., "U1trasonic Thermometry for Nuclear Reactors", IEEE Trans. Am. Nuc. Soc., NS-16, February 1969, pp. 184-187.

22. C. R. Hann, et. a1., Data Report for the NRC/PNL Halden Assemb1y IFA-431, PNL-2494, Battelle, Pacific Northwest Laboratories, Richland, WA 99352 , June 1978. 
APPENDIX A

FUEL ROD AND FUEL COLUMN SCHEMATICS FOR IFA-432 
. 


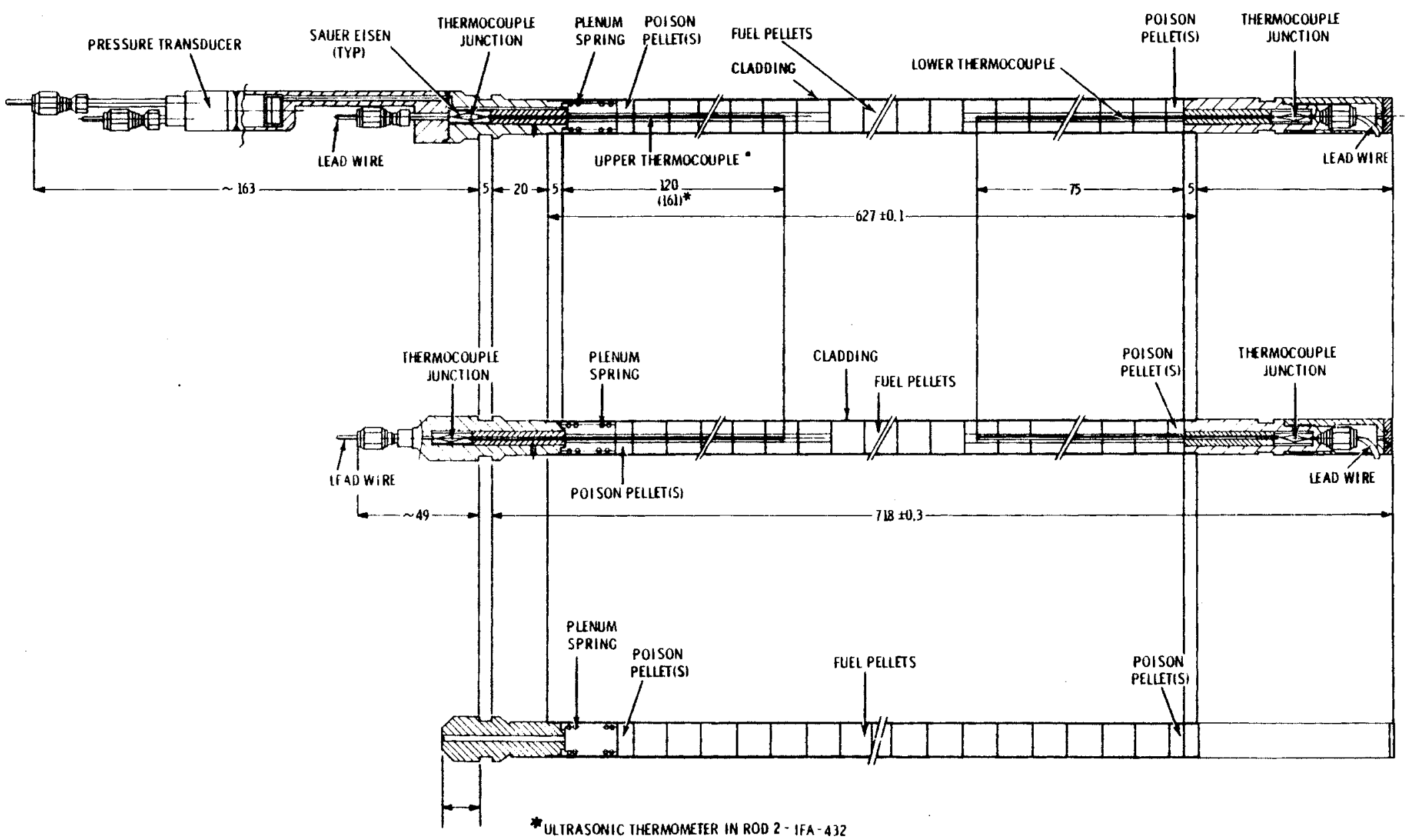

FIGURE A-1. Schematic Arrangement of Fuel Rods for IFA-432 


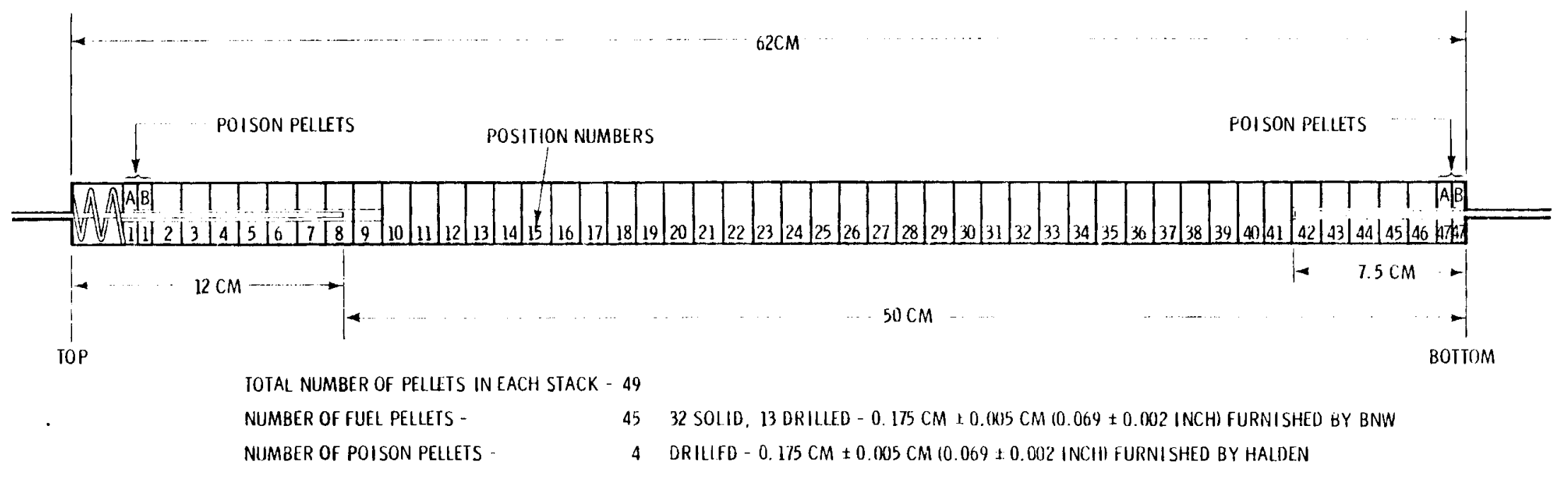

\begin{tabular}{|c|c|c|c|c|c|}
\hline ROD NO. & \multicolumn{2}{|c|}{ PELIET DIAMEIER } & $\begin{array}{l}\text { FUEL DENSITY } \\
\% \text { ID }\end{array}$ & FUEL TYPE & $\begin{array}{l}\text { FILLGAS } \\
1 \text { AIM }\end{array}$ \\
\hline 1 & & & 95 & & \\
\hline 6 & $1.0681(0.4205)$ & $1.0681(0.4205)$ & 92 & UNSTABLE & HELIUM \\
\hline
\end{tabular}

FIGURE A-2. Stack Arrangement for Rods 1 and 6 


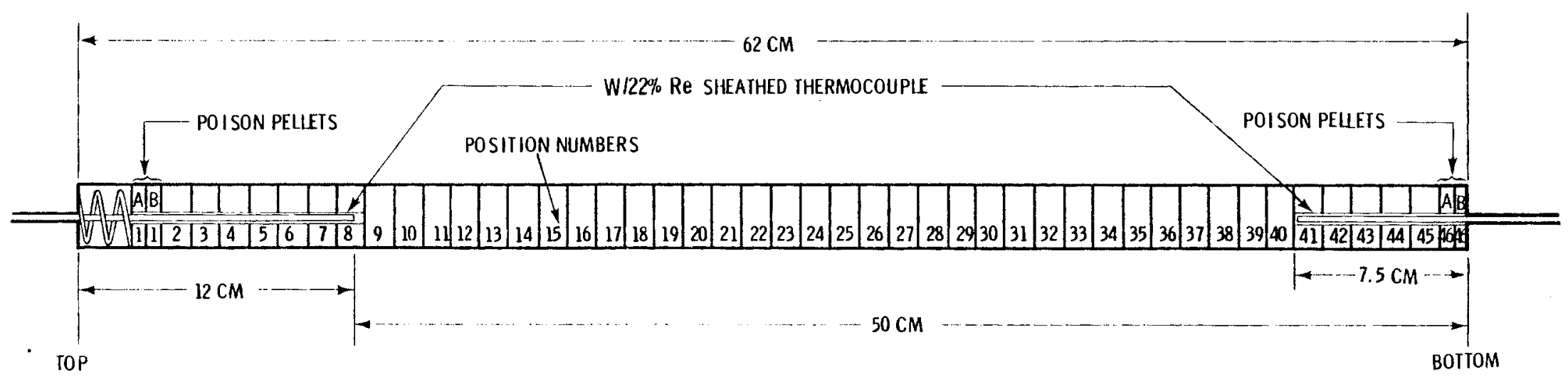

$\stackrel{\$}{1}$

TOTAL NUMBER OF PELLETS IN EACH STACK - 48

NUMBER OF FUEL PELLETS -

4432 SOLID, 12 DRILLED $-0.175 \pm 0.005 \mathrm{CM}(0.069 \pm 0.002$ INCH) FURNISHED BY BNW

NUMBER OF POISON PELLETS -

4 DRILLED $-0.175 \pm 0.005 \mathrm{CM}(0.069 \pm 0.002$ INCH) FURNISHED BY HALDEN

\begin{tabular}{|c|c|c|c|c|c|}
\hline \multirow[b]{2}{*}{ ROD NO. } & \multicolumn{2}{|c|}{ PELLET DIAMETER } & \multirow[b]{2}{*}{$\begin{array}{l}\text { FUEL DENSITY } \\
\% \text { ID }\end{array}$} & \multirow[b]{2}{*}{ FUEL TYPE } & \multirow[b]{2}{*}{$\begin{array}{l}\text { FILL GAS } \\
1 \text { ATM }\end{array}$} \\
\hline & $\begin{array}{l}\text { IFA-431 } \\
\text { CM (inch) }\end{array}$ & $\begin{array}{l}\text { IFA-432 } \\
\text { CM (inch) }\end{array}$ & & & \\
\hline 2 & $1.0528(0.4145)$ & $\cdots$ & 95 & LE. & HELIUM \\
\hline 3 & $1.0858(0.4275)$ & $1.0833(0.4265)$ & 95 & SIABLE & HELIUM \\
\hline 5 & $1.0681(0.4205)$ & $1.0681(0.4205)$ & 92 & STABIF & HELIUM \\
\hline
\end{tabular}

FIGURE A-3. Stack Arrangement for Rods 2, 3, and 5 


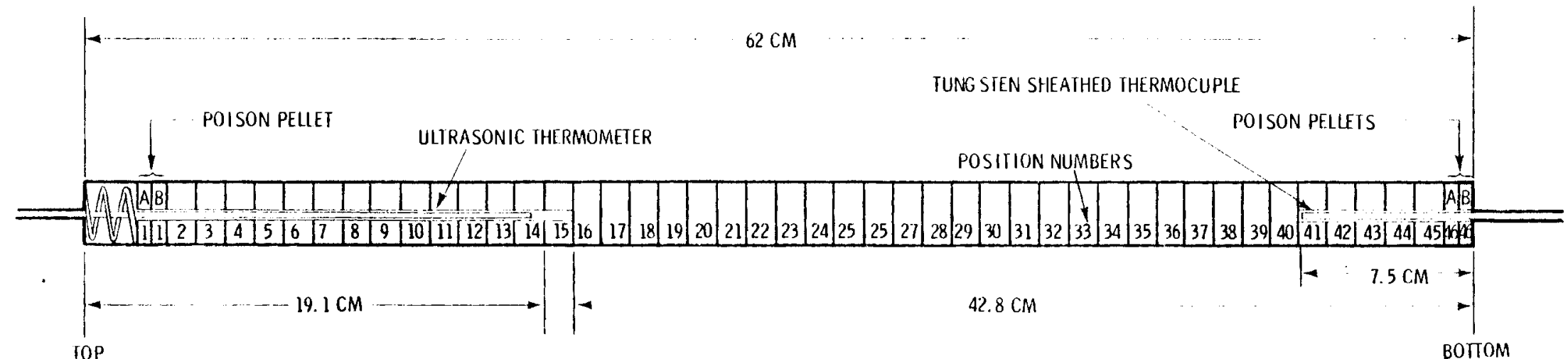

TOTAL NUMBER OF PELLETS IN EACH SIACK - 48 NUMBER OF FUEL PELLETS -

4432 SOLIO, 12 DRILLED $-0.175 \pm 0.005 \mathrm{CM}(0.069 \pm 0.002$ INCH) FURNISHED BY BNW NUMBER OF POISON PELLEIS 4 DRILLEO $-0.175 \pm 0.005 \mathrm{CM}(0.069 \pm 0.002$ (NCH) FURNISHED BY HALDEN

$$
\frac{\text { ROD NO. }}{2} \frac{\begin{array}{c}
\text { PELIET DIAMETER } \\
\text { IFA-432 } \\
\text { CM(INCH) }
\end{array}}{1.0528(0.4145)} \frac{\begin{array}{c}
\text { FUEL DENSITY } \\
\% \text { ID }
\end{array}}{95}-\frac{\text { FUEL TYPE }}{\text { STABLE }} \frac{\begin{array}{c}
\text { FILL GAS } \\
1 \text { ATM }
\end{array}}{\text { HELIUM }}
$$

FIGURE A-4. Stack Arrangement for Rod 2, IFA-432 


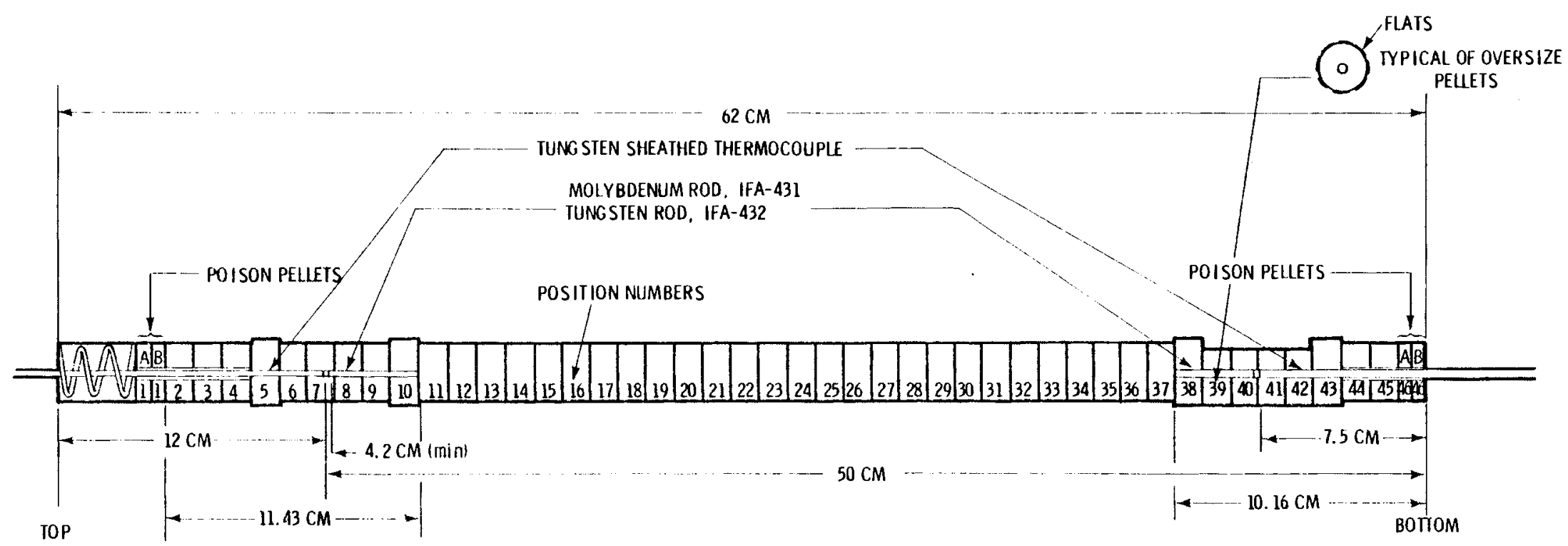

TOTAL NUMBER OF PELLETS IN STACK - 48 NUMBER OF FUEL PELLETS -

4427 SOLID, 17 DRILLED (FURNISHED BY BNW), 95\% TD, STABLE

NUMBER OF POISON PELLEIS -

4 DRILLED $-0.175 \pm 0.005 \mathrm{CM}(0.069 \pm 0.002$ INCH) (FURNI SHED BY HALDEN)

FILL GAS -

1 ATM XENON

\begin{tabular}{|c|c|c|c|c|c|}
\hline $\begin{array}{l}\text { PELLET } \\
\text { POSIIION } \\
\end{array}$ & $\begin{array}{l}\text { PELLET D } \\
\text { IFA-431 } \\
\text { CM (inch) } \\
\end{array}$ & $\begin{array}{l}\text { IAMETER } \\
\text { IFA-432 } \\
\text { CM (inch) } \\
\end{array}$ & $\begin{array}{l}\text { HOLE DIAMETER } \\
\text { CM (inch) }\end{array}$ & ON CENTER & $\begin{array}{c}0.0127 \mathrm{CM}(0.005 \text { (NCH) } \\
\text { OFF CENIER }\end{array}$ \\
\hline $12-38$ & $1.0681(0.4205)$ & $1.0681(0.4205)$ & -- & -- & -- \\
\hline $\begin{array}{r}3-5 \\
45-46\end{array}$ & $1.0681(0.4205)$ & $1.0681(0.4205\}$ & $\begin{array}{r}0.175 \pm 0.005 \\
(0.069 \pm 0.002)\end{array}$ & YES & - \\
\hline $7-10$ & $1.0681(0.4205)$ & $1.0687(0.4205)$ & $\begin{array}{c}0.160 \pm 0.005 \\
(0.063 \pm 0.002)\end{array}$ & YES & - \\
\hline $40-43$ & $1.0681(0.4205)$ & $1.0681(0.4205)$ & $\begin{array}{c}0.160 \pm 0.005 \\
(0.063 \pm 0.002)\end{array}$ & -- & YES \\
\hline $\begin{array}{l}6,11, \\
39,44\end{array}$ & $1.0858(0.4275)$ & $1.0833(0.4265)$ & $\begin{array}{c}0.160 \pm 0.005 \\
10.063 \pm 0.002\end{array}$ & YES & - \\
\hline
\end{tabular}

FIGURE A-5. Stack Arrangement for Rod 4 - Xenon Fill Gas 


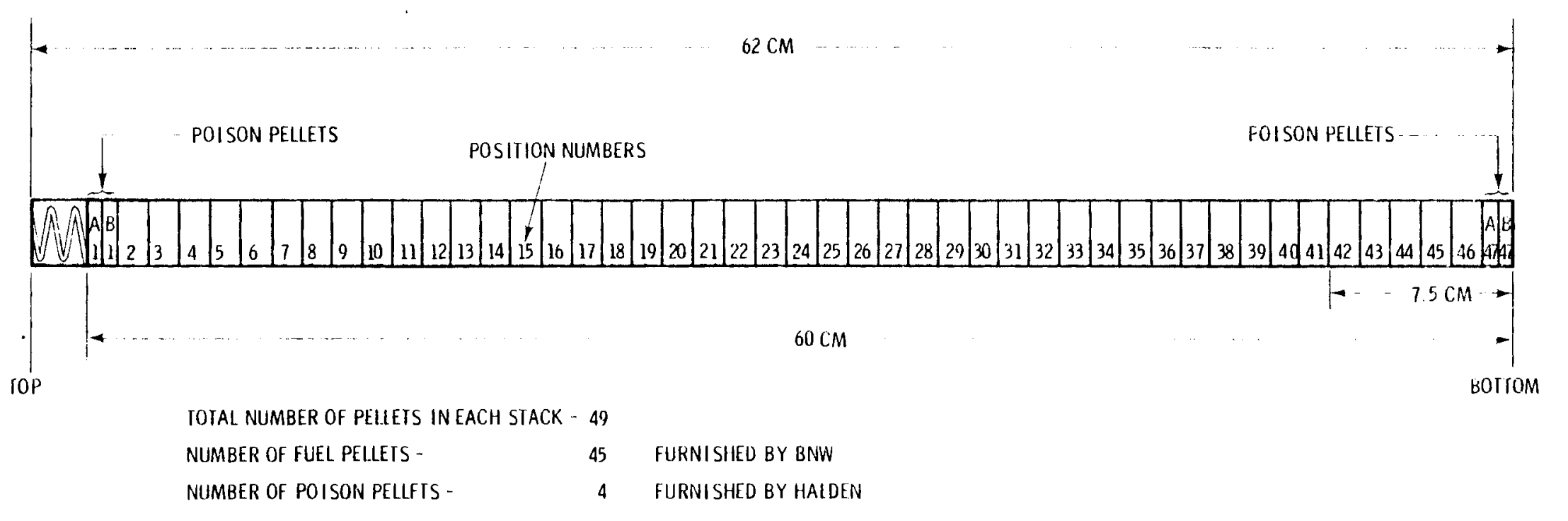

\begin{tabular}{|c|c|c|c|c|}
\hline ROD NOJ. & $\begin{array}{l}\text { PELLET DIAMETER } \\
\text { IFA-432 } \\
\text { CM (INCH) }\end{array}$ & $\begin{array}{l}\text { FUEL DENSITY } \\
\% \mathrm{TD}\end{array}$ & FUEL IYPE & $\begin{array}{l}\text { FIILGAS } \\
\text { IAIM }\end{array}$ \\
\hline & $1.0528(0.4145)$ & 95 & SIABLE & HEIIUUM \\
\hline 8 & $1.0681(0.4205)$ & 95 & STABIE & HELIUM \\
\hline 9 & $1.0732(0.4225)$ & 95 & SIABIE & HELIUM \\
\hline
\end{tabular}

FIGURE A-6. Stack Arrangement for Non-Instrumental Replacement Rods 7, 8, and 9 IFA-432 
APPENDIX B

INSTRUMENT DESCRIPTIONS AND CALIBRATION 
APPENDIX B

INSTRUMENT DESCRIPTIONS AND

CALIBRATION

IFA-432 was equipped with a comprehensive array of in-pile instrumentation to collect the data. The placement of these instruments was shown in Fig-. ures 2.1 and 2.2. The most important of these instruments were:

- Six vanadium beta emitter self-powered neutron detectors

- One cobalt fast response self-powered neutron detector

- Eleven W5\%Re/W26\%Re sheathed fuel centerline thermocouples

- One U1trasonic thermometer

- Six LVDT cladding elongation monitors

- Three diaphragm-type rod internal pressure transducers.

Each of these is briefly discussed below. The accuracy and uncertainty of their respective outputs is discussed more completely in Reference 19.

\section{NEUTRON DETECTORS}

IFA-432 was equipped with six vanadium, self-powered, beta current neutron detectors (Figure $B-1$ ) to monitor the power in the fuel assembly after the initial thermal-hydraulic calibration.

Each vanadium neutron detector was $3.93 \mathrm{in}$. $(100 \mathrm{~mm})$ long and was positioned so that the center of the detector and the thermocouple junction were located on essentially the same plane.

The neutron detectors used in IFA-432 were not calibrated. Their precisions were based on the results of the irradiation of 30 similar type vanadium neutron detectors in the Studsvik R2-0 Reactor in Sweden. The 30 detectors were irradiated in a thermal neutron flux of $1.1 \times 10^{10} \mathrm{n} / \mathrm{cm}^{2}-\mathrm{sec}$. The error 1 imits for the outputs of the detectors were estimated to be $\pm 2.5 \%$ at a neutron flux of $1.1 \times 10^{10} \mathrm{n} / \mathrm{cm}^{2}-\mathrm{sec}$. 


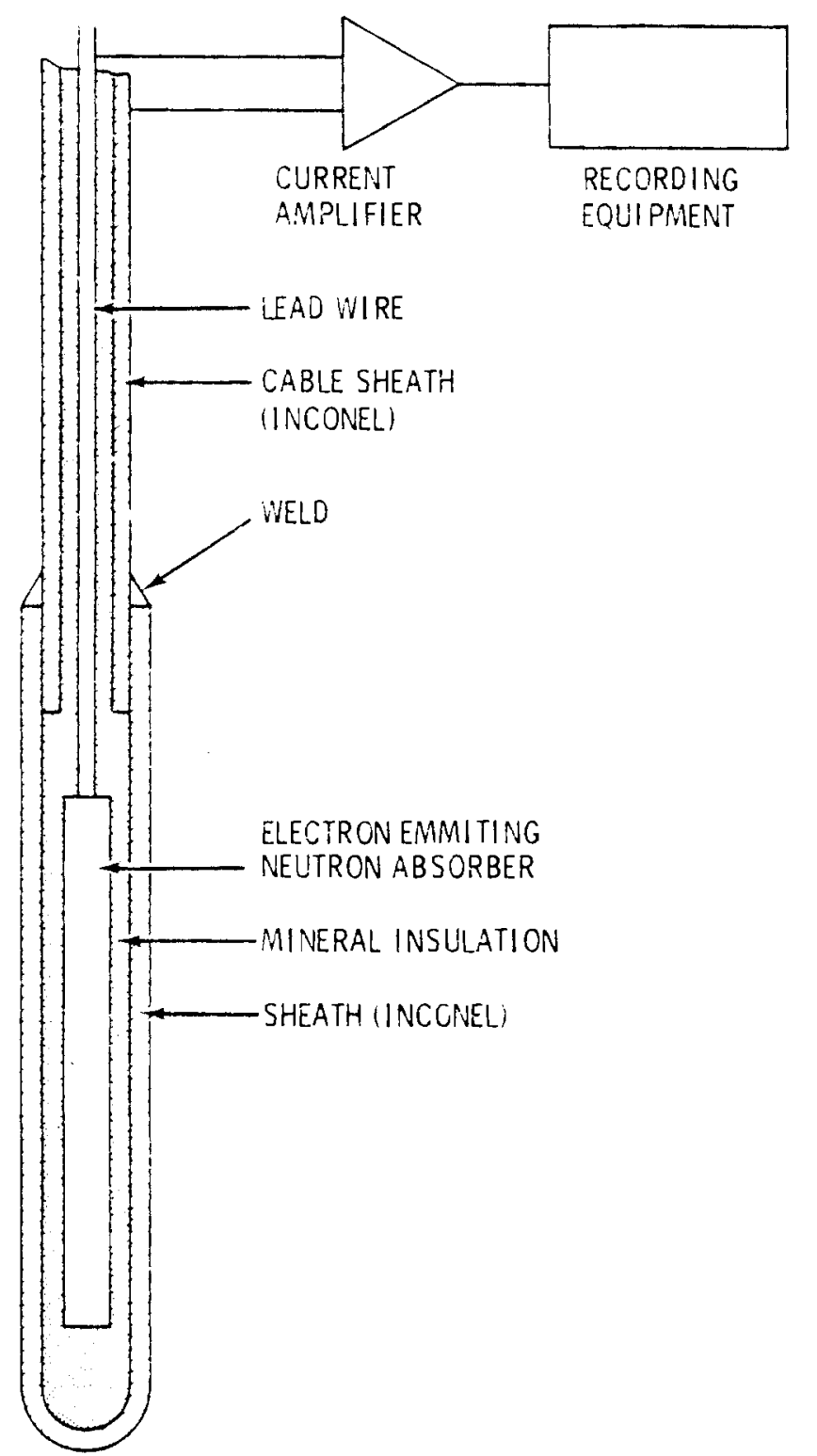

FIGURE B-1. Schematic of Self-Powered, Beta Current Neutron Detector 
In addition to correlating the detector outputs to the neutron flux in the Studsvik reactor, Halden has conducted long-term tests of similar neutron detectors in the HBWR. These long-term tests have established the detectors as reliable and accurate instruments without a measurable change in sensitivity at the higher flux levels in the HBWR.

The sensitivities of the test assembiy neutron detectors were calculated from the sensitivities of the calibrated detectors and the physical characteristics of the test assembly detectors supplied by the manufacturer. The gamma sensitivity is not measured and is considered by Halden to be negligable.

The vanadium detectors have a calculated burnup rate of $0.013 \%$ month at a neutron flux of $1 \times 10^{13} \mathrm{n} / \mathrm{cm}^{2}-\mathrm{sec}$. Based on this rate, the neutron detector end-of-life (EOL) burnup for IFA-432 is $0.3 \%$. Because of this low value, the neutron detector outputs were not corrected for burnup.

A disadvantage of the vanadium detectors is their slow response time $(5.5 \mathrm{~min}, 0$ to $63 \%)$ to power changes. Consequentiy, during up and down power ramps, a correction factor should be considered for the output values.

The cobalt detector, similar in appearance but $20 \mathrm{~cm}$ long, was placed in the center of the assembly to monitor average assembly power during transient tests. (6)

\section{FUEL THERMOCOUPLES}

Eleven thermocouples were used in IFA-432 for measuring the central fuel temperatures. The thermocouples had grounded junctions with 0.062 in. $(1.575 \mathrm{~mm})$ OD tungsten $/ 22 \%$ rhenium sheaths and W $5 \%$ Re/W $26 \%$ Re seven-stranded thermocouple wires with thorium oxide insulators (Figure B-2). The sensor in the top of Rod 2 was an ultrasonic thermometer (Reference 21). It failed early in life.

The thermocouples were fabricated and calibrated by the Idaho National Engineering Laboratory (INEL). The calibration curve for the tungstenrhenium thermocouples is shown in Figure B-3. Calibration of the thermocouples over the range of use produces a brittle assembly which is fragile and subject 


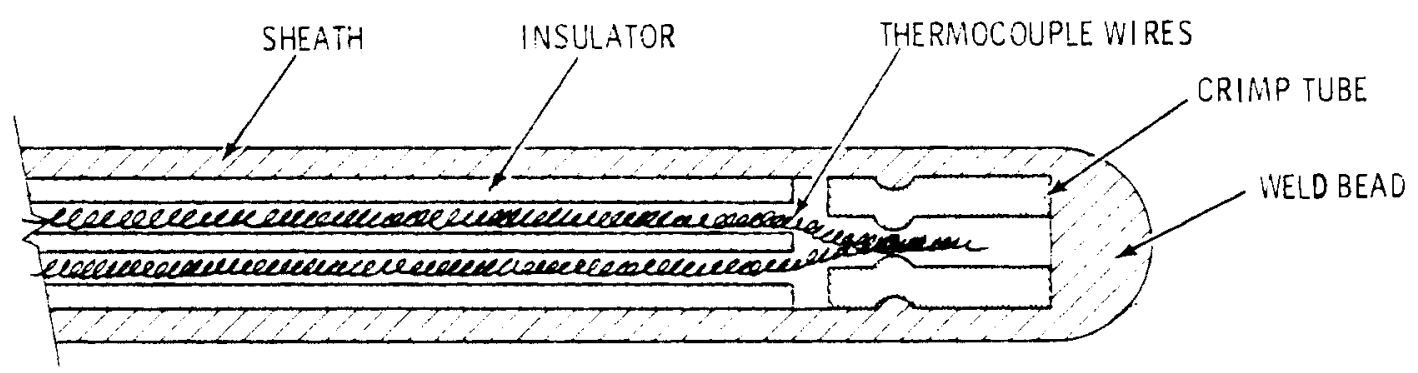

FIGURE B-2. Schematic of W $5 \%$ Re/W $26 \%$ Re Thermocuples with Grounded Junction

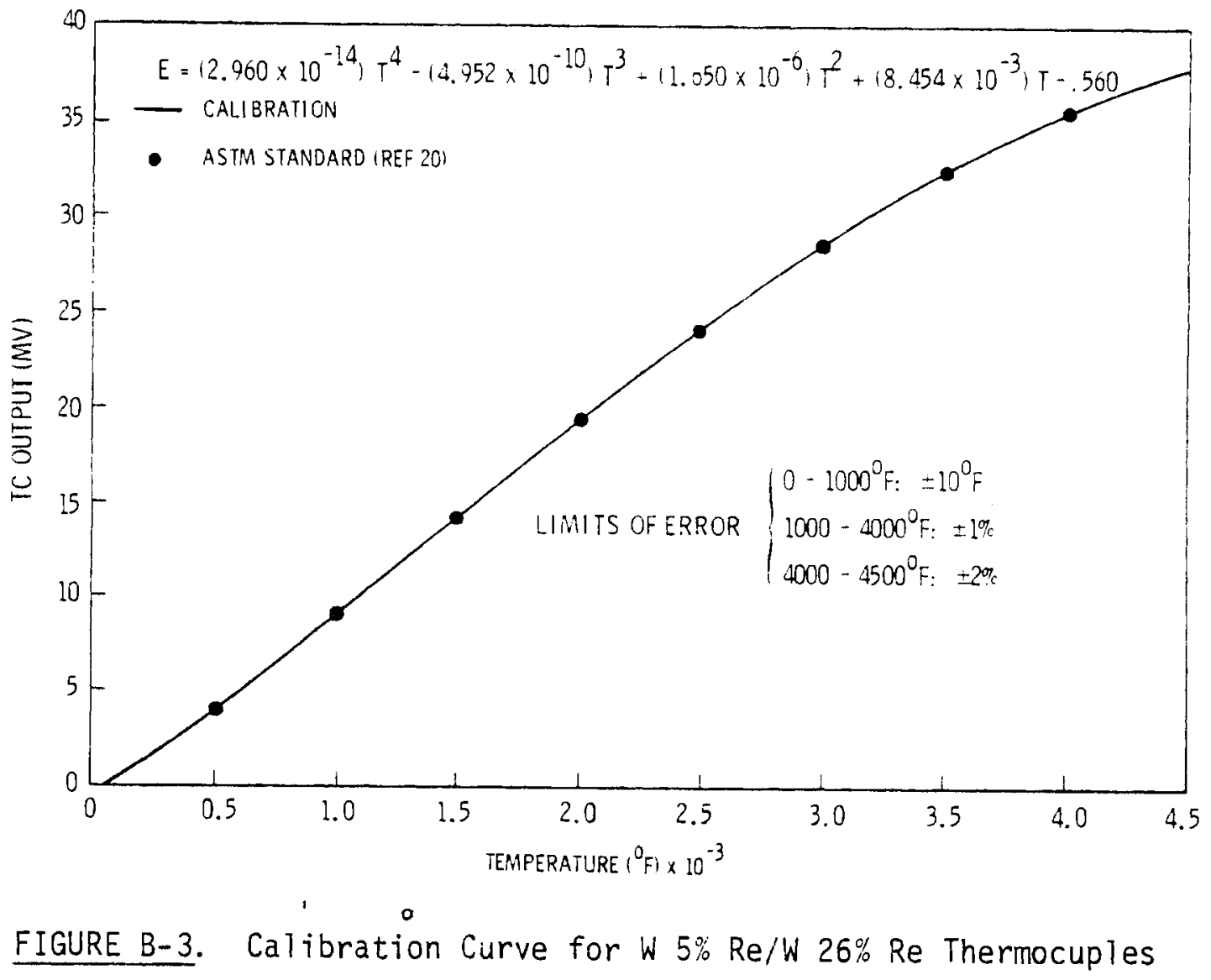


to breakage. Consequently, only one thermocouple, which was not used in the in-reactor test, was calibrated.

The tungsten rhenium thermocouple was calibrated against a reference thermocouple of bare $\mathrm{W} 5 \% \mathrm{Re} / \mathrm{W} 26 \%$ Re and an optical pyrometer as a second reference. The reference thermocouple and the optical pyrometer agreed with in $40^{\circ} \mathrm{F}\left(22^{\circ} \mathrm{C}\right)$ up to $4000^{\circ} \mathrm{F}\left(2204^{\circ} \mathrm{C}\right)$, but as the temperature approached $4500^{\circ} \mathrm{F}$ $\left(2482^{\circ} \mathrm{C}\right)$, the two differed more widely. The optical pyrometer was thought to be closer since the $4500^{\circ} \mathrm{F}$ temperature is above that given in most calibration tables for $W / R e$ thermocouples. The calibrated thermocouple had the following limits of error:

$$
\begin{array}{ll}
\text { Ambient to } 1000^{\circ} \mathrm{F}\left(538^{\circ} \mathrm{C}\right) & = \pm 10^{\circ} \mathrm{F}\left(5.5^{\circ} \mathrm{C}\right) \\
1000^{\circ} \mathrm{F}\left(538^{\circ} \mathrm{C}\right) \text { to } 4000^{\circ} \mathrm{F}\left(2204^{\circ} \mathrm{C}\right) & = \pm 1 \% \text { of reading } \\
4000^{\circ} \mathrm{F}\left(2204^{\circ} \mathrm{C}\right) \text { to } 4500^{\circ} \mathrm{F}\left(2482^{\circ} \mathrm{C}\right) & = \pm 2 \% \text { of reading. }
\end{array}
$$

Irradiation of the thermocouples will have long term effects caused by the shunting of the EMF's by conduction across the insulators, by transmutations in the thermocouple materials, and by temperature gradients along the thermocouple wires. The insulator shunting effect was reduced to a negligible level by using thorium oxide insulators.

The expected fluence for IFA-432 is about $5 \times 10^{20}$ nvt. This fluence would indicate a downward EMF drift of 5\% for IFA-432 at EOL. No correction factor is applied to the IFA-432 data. However, an error value of $\pm 5 \%$ is assumed for the irradiation effects.

\section{CLADDING ELONGATION MONITORS}

Figure B-4 is a schematic of the LVDT cladding elongation sensors used in IFA-432. These instruments are mounted upside down at the bottom of the assembly, the core extension contacting the lower end plug of the rod. The ferromagnetic core is attached to the extension and moves inside a coil system with the central primary coil carrying $50 \mathrm{~mA} 400 \mathrm{~Hz}$ excitation. A secondary coil consisting of two balanced halves flanks the primary coil. The output voltage is zero when the core is in its central position, and increases linearly when the cladding elongation moves the core. Sample calibration curves for these instruments may be found in Reference 22 . 


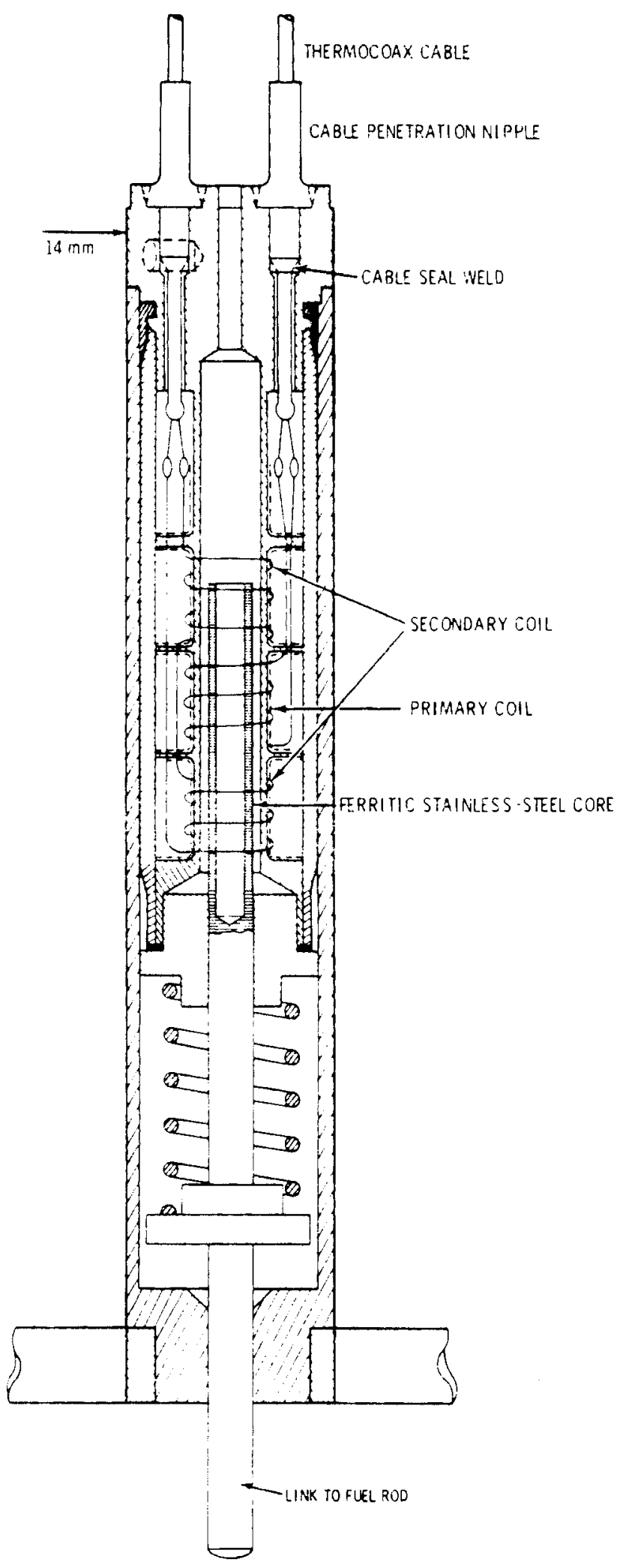

FIGURE 3-4. Cladding Elongation Monitor (HaTcien Project Design) 


\section{FISSION GAS PRESSURE TRANSDUCERS}

Figure B-5 shows a schematic of the diaphragm-type pressure transducer used to measure the internal rod pressures due to fission gas release during irradiation. This is essentially an on-off type measurement. The thin platinum alloy membrane is exposed to the rod internal gases on one side, while the other side is connected to an external pressure manifold. When the external pressure equals the internal pressure, the deflection of the membrane causes it to make an electrical contact. The step increase in voltage signals a null pressure balance: Over a range of $100 \mathrm{~kg} / \mathrm{cm}^{2}$, the instrument's sensitivity is $0.1 \mathrm{~kg} / \mathrm{cm}^{2}$. The accuracy and repeatability are $\pm 1 \mathrm{~kg} / \mathrm{cm}^{2}$ and $\pm 0.4 \mathrm{~kg} / \mathrm{cm}^{2}$, respectively.

Calibration is out-of-reactor and consists of checking the deflection sensitivity of the membrane, which does not change appreciabiy with pressure level. The effects of irradiation on the membrane are not known, but are assumed by Halden to be minimal. Halden has made no recommendation for temperature compensation for this instrument. Any temperature compensation deemed necessary would be due to differential thermal expansion between the membrane and gage body, and to the temperature level of the membrane itself. Table B-i is provided for this purpose. It lists coolant temperatures corresponding to the pressure measurements when the coolant temperature was not $235 \pm 10^{\circ} \mathrm{C}$. Details of the pressure transducer must be obtained from the Halden Project. 


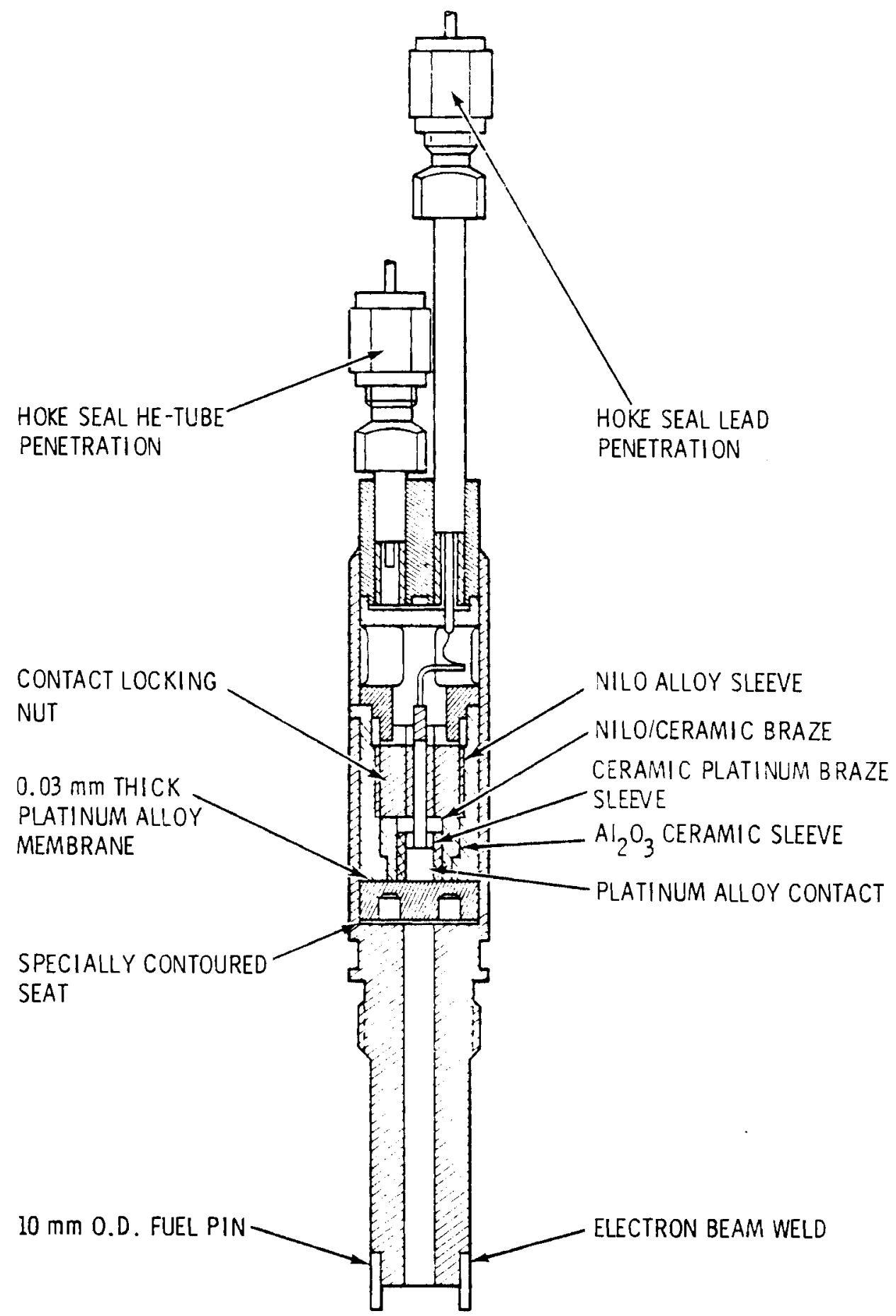

FIGURE B-5. Fission Gas Pressure Transducer (Halden Project Design) 
TABLE B-1. IFA-432 Coolant Temperatures other Than $235 \pm 10^{\circ} \mathrm{C}$

\begin{tabular}{|c|c|c|}
\hline Date & Time & Moderator Temperature $\left({ }^{\circ} \mathrm{C}\right)$ \\
\hline $11-30-75$ & 1235 & 76.0 \\
\hline $12-1-75$ & 745 & 150.0 \\
\hline $12-1-75$ & 1930 & 153.6 \\
\hline $12-2-75$ & 845 & 208.0 \\
\hline $12-9-75$ & 215 & 221.0 \\
\hline $12-30-75$ & 1230 & 222.0 \\
\hline $1-7-76$ & 1015 & 220.0 \\
\hline $1-4-77$ & 626 & 222.7 \\
\hline $3-24-77$ & 1247 & 174.5 \\
\hline $3-25-77$ & 1628 & 203.1 \\
\hline $5-21-77$ & 28 & 199.6 \\
\hline $5-21-77$ & 218 & 198.9 \\
\hline $5-21-77$ & 1840 & 68.8 \\
\hline $5-21-77$ & 2056 & 70.4 \\
\hline $5-22-77$ & 1950 & 70.6 \\
\hline $6-16-77$ & 1931 & 79.0 \\
\hline $6-19-77$ & 2250 & 183.3 \\
\hline $6-22-77$ & 1556 & 73.0 \\
\hline $6-23-77$ & 555 & 153.2 \\
\hline $6-24-77$ & 921 & 215.7 \\
\hline $6-24-77$ & 1543 & 213.5 \\
\hline $6-28-77$ & 2234 & 200.2 \\
\hline $7-7-77$ & 1209 & 145.2 \\
\hline $7-15-77$ & 1309 & 206.5 \\
\hline $8-3-77$ & 1420 & 216.1 \\
\hline $8-3-77$ & 1816 & 211.6 \\
\hline $8-5-77$ & 1428 & 215.1 \\
\hline $8-26-77$ & 1607 & 220.6 \\
\hline $8-26-77$ & 1659 & 220.1 \\
\hline $10-1-77$ & 1941 & 73.0 \\
\hline $10-2-77$ & 2307 & 167.8 \\
\hline $10-3-77$ & 1806 & 197.1 \\
\hline $10-7-77$ & 845 & 186.3 \\
\hline $10-7-77$ & 906 & 189.5 \\
\hline
\end{tabular}


TABLE B.2. Instrument Failure Dates for IFA - 432

$\begin{array}{lllcc}\frac{\text { Rod }}{1} & \frac{\text { UTC }}{\text { January 31, 1977 }} & & \text { LTC } & \text { PT } \\ 2 & \text { STARTUP } & & \text { NA } \\ 3 & & & \text { NA } & \text { (a) } \\ 4 & \text { December 31, 1975 } & \text { December 31, 1975, 1976 } & \text { NA } \\ 5 & \text { June 30, 1976 } & & \text { February 14, 1976 } \\ 6 & \text { November 31, 1976 } & & & \end{array}$

(a) Note that Rod 4 was withdrawn on February 20, 1976 and replaced with the non-instrumented Rod 8 (see Table 2.1). Cladding extensometer readings are for Rod 8 after this date. 


\section{APPENDIX C}

ASSEMBLY POWER CALIBRATION 
. 


\section{ASSEMBLY POWER CALIBRATION}

The data report for the IFA-431 assembly (PNL-2494) explained briefly the usual method for calibrating assemblies in the Halden reactor. This procedure was not used in the case of IFA-432 since the calibration flow valve (Figure 2.2) failed in the normal operating position, allowing only natural circulation. However, both assemblies were in core simultaneously at the time of IFA-432 startup. The second assembly was calibrated by comparisons of total assembly power and Rod 3 (smal1 gap) power. The uncertainty in assembly power for IFA-432 is estimated to be $\pm 6 \%$. 


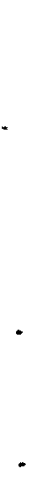


APPENDIX D

DATA PROCESSING 
APPENDIX D

DATA PROCESSING

Processing of the data received from Halden on magnetic tape proceeds as shown in Figure D-1. After reception of the data tape, it must be translated from the Halden IBM/1800 language (EBCDIC) to the PDP11/45 language (ANSI), and the translated version stored on tape. Then this tape is reformatted so that all the data from a particular time on a particular date is in one block. This is simultaneously stored on a disc file.

Once the raw data is stored on disc, another program (Figure D-2) is applied to each block of data to correct the rod local heat ratings at the thermocouple locations for radial flux tilt across the assembly. This was necessary for IFA-432 since the powers on the original data tape actually represented the powers at the neutron detector locations and not at the thermocouple locations (Figure 2.2). Rod local and assembly powers are also corrected for axial flux shape and heat losses to the moderator. Corrections for local mass distributions of fissile material for each rod are also made. Table D-1 indicates typical values for these correction factors.

While this is being done, other checks are being made on the data. For the assembly and rod average powers, a total heat balance check is made. This is done during application of the axial correction factor to account for the difference between the average and true mean of the axial flux distribution. The first attempt at this uses an axial profile that represents normal operating conditions. If the heat balance for this profile does not check, an error message is printed and a second attempt is made with an axial flux shape that represents a disturbed flux profile. This occurs when a nearby control rod is partially inserted. In nearly all cases, the normal profile produces a heat balance that checks to within less than $1 / 2 \%$.

When this step is complete, another program corrects the burnups and heat ratings for depletion of ${ }^{235} \mathrm{U}$. Plutonium buildup is not taken into account. 


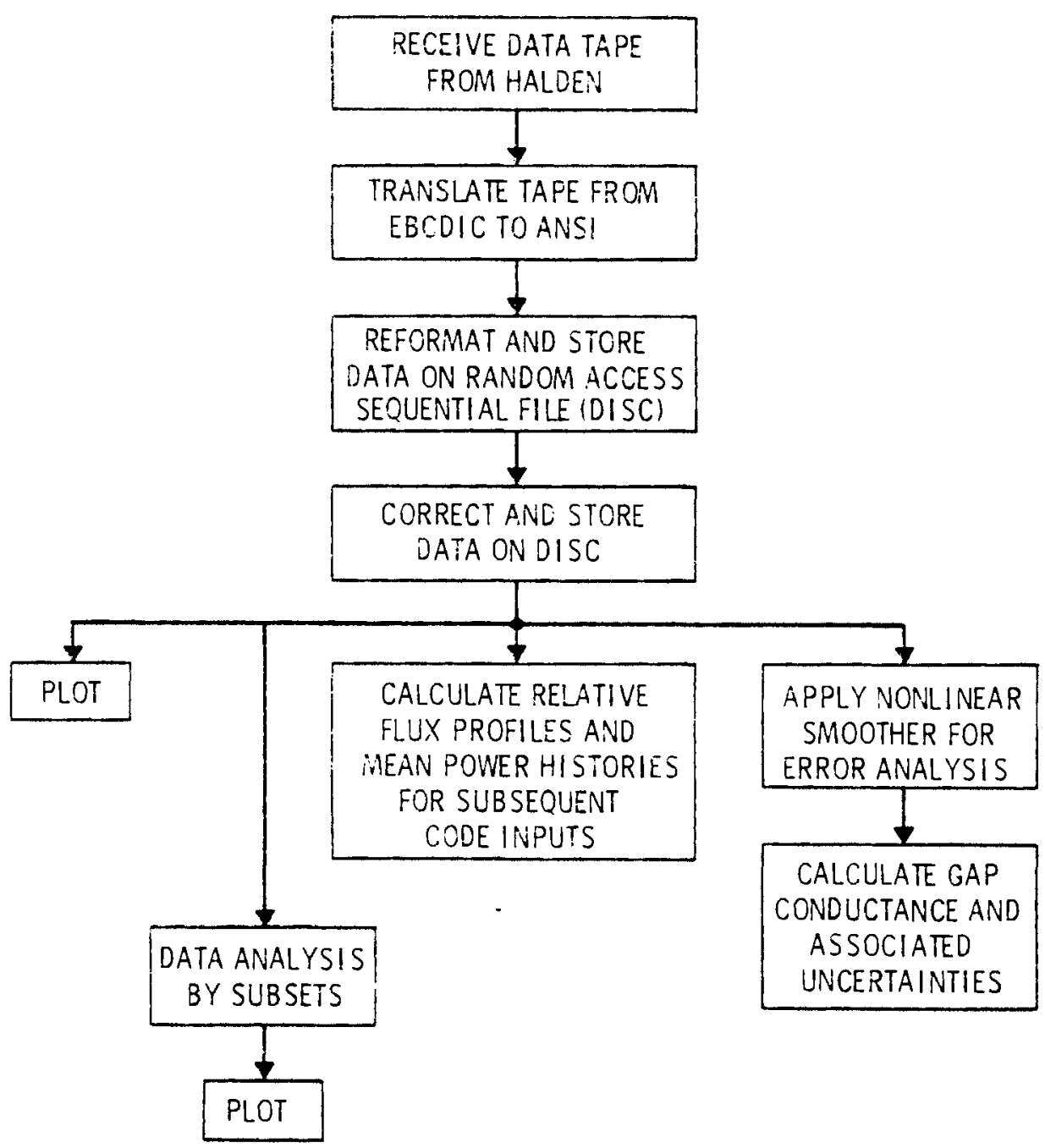

FIGURE D-1. Flow Diagram for Processing Halden Data

TABLE D-1. Typical Power Correction Factors for IFA-432

\begin{tabular}{|c|c|c|c|c|c|c|}
\hline Rod & Fuel & $\begin{array}{c}\text { Radial } \\
\text { Tilt } \\
\end{array}$ & $\begin{array}{l}\text { Axial } \\
\text { Shape }\end{array}$ & $\begin{array}{l}\text { Neutron } \\
\text { Detector } \\
\text { Average } \\
\end{array}$ & $\begin{array}{c}\text { Gamma } \\
\text { Heating }\end{array}$ & Tota 1 \\
\hline 1 & 1.0190 & 1.106 & 0.952 & 1.038 & 0.988 & 1.1003 \\
\hline 2 & 0.9628 & 0.945 & 0.952 & 1.038 & 0.988 & 0.8883 \\
\hline 3 & 1.0614 & 0.926 & 0.052 & 1.038 & 0.988 & 0.9596 \\
\hline 4 & 1.0044 & 1.125 & 0.952 & 1.038 & 0.988 & 1.1032 \\
\hline 5 & 0.9797 & 0.987 & 0.952 & 1.038 & 0.988 & 0.9435 \\
\hline 6 & 0.9878 & 0.957 & 0.952 & 1.038 & 0.988 & 0.9229 \\
\hline
\end{tabular}

Burnup Correction $=-0.56 \%$ per $1000 \mathrm{MWD} / \mathrm{MTM}$ 


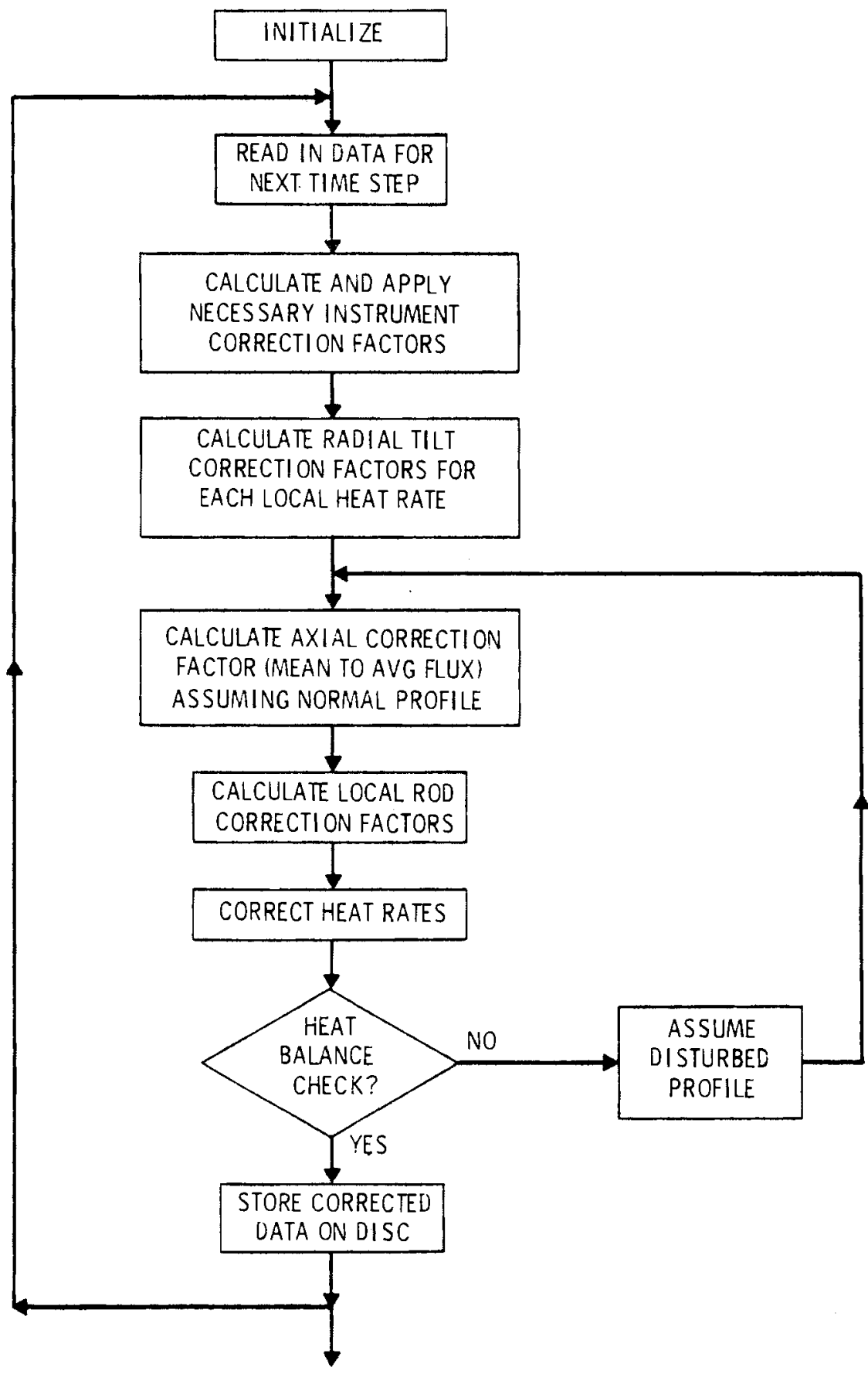

FIGURE D-2. Flow Diagram for Correction of Halden Rod Local Powers 
After the data are corrected, four paths are possible and usually are executed simultaneously. The data are plotied, reiative flux profiles and mean power histories are calculated for input to safety related codes, an error analysis is performed on the corrected data, and the data is analyzed by subsets (i.e., transient, steady state) to separate out the effects of the major variables. A sample of the relative axial power profile seen by the assembly is shown in Figure D-3. Figure D-4 is a flow diagram of the gap conductance and uncertainty calculations performed. (19) 


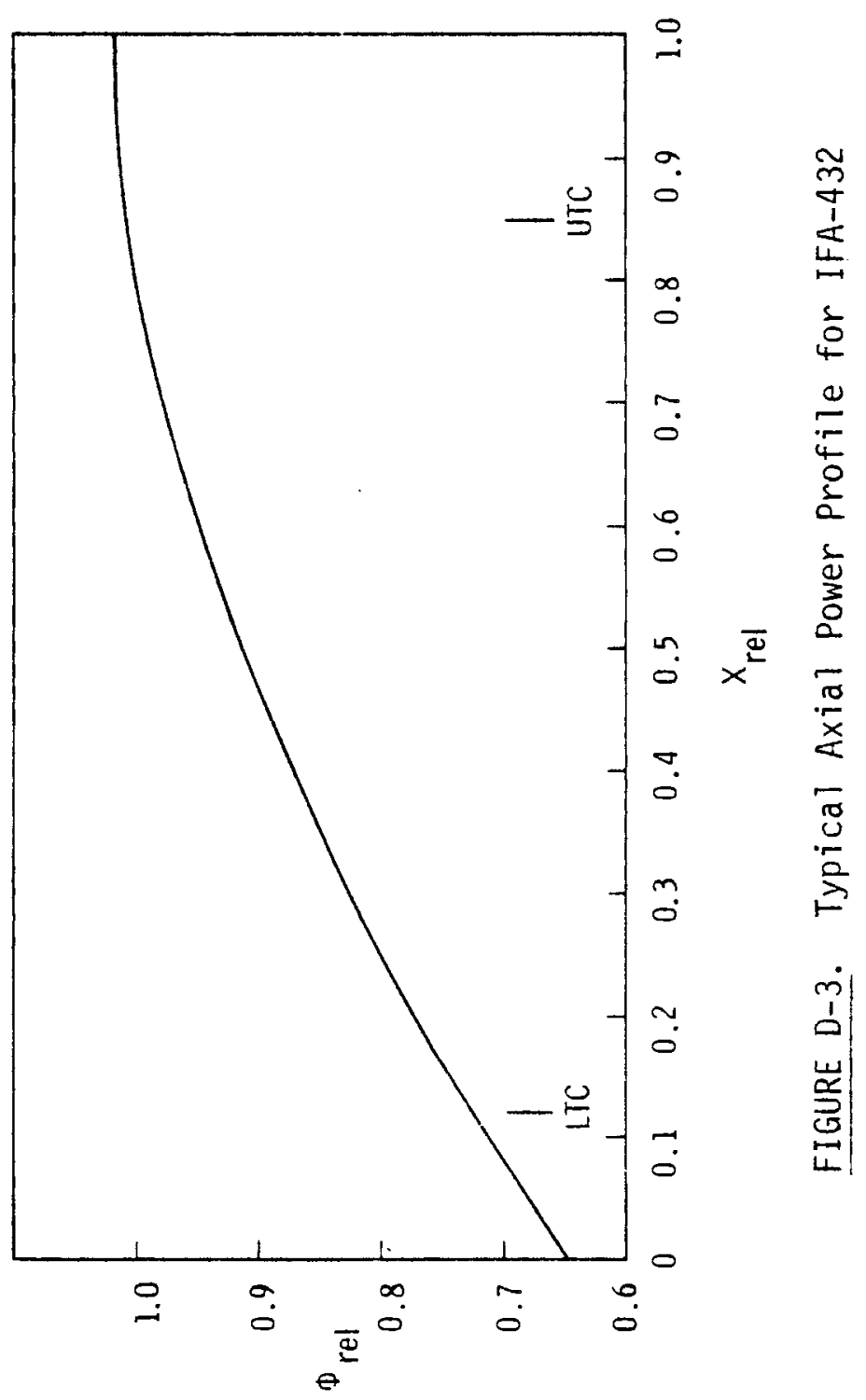




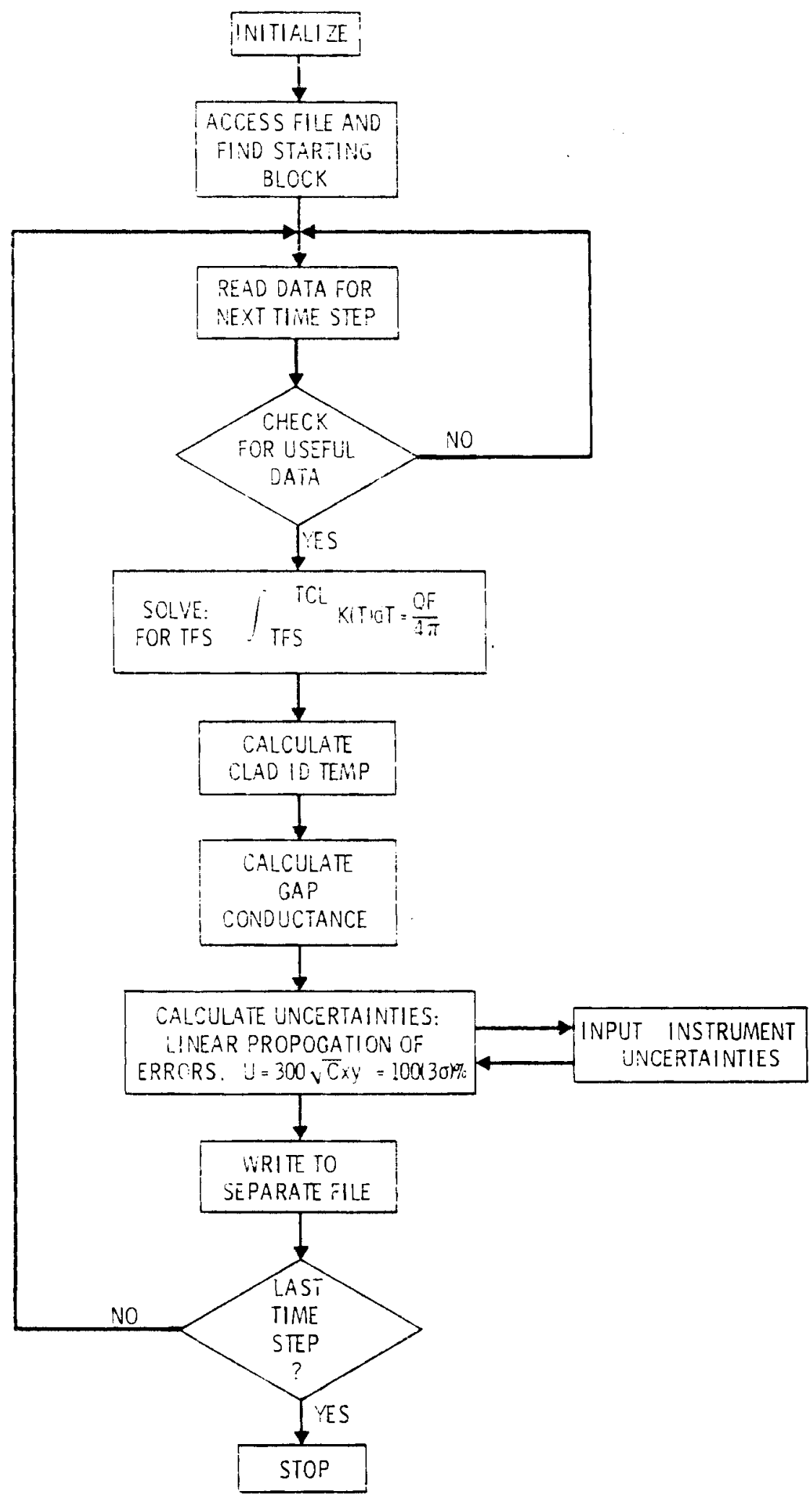

FIGURE D-4. Flow Diagram for Gap Conductance and Uncertainty Calculations 


\section{DISTRIBUTION}

No. of

Copies

OFFSITE

10 Chief, Fuel Behavior Research Branch

Division of Reactor Safety Research

U.S. Nuclear Regulatory

Commission

Washington, D.C 20555

Librarian, Technical Lib.

Argonne National Laboratory

9700 South Cass Avenue

Argonne, IL 60439

Manager, Product Developmt. Division

Babcock \& Wilcox Company

P.O. Box 1260

Lynchburg, VA 24505

R. Duncan

Vice-President - Devlopmt.

Nuclear Power Systems

Combustion Engineering, Inc.

P.0. Box 500

Windsor, CT 06095

Manager

Code Verification \& App1. Br.

EG\&G Idaho, Inc.

P.0. Box 1625

Idaho Falls, ID 83401

2 DOE Technical Information Center
No. of

Copies
J. T. A. Roberts

Electric Power Research

Institute

3412 Hillview Avenue

Palo Alto, CA 94304

Manager

Fuel Technology

Exxon Nuclear Company, Inc. 2955 George Washington Way

Richland, WA 99352

Manager

Program Development \&

Evaluation

Thermal Fuel Behavior Div.

EG\&G Idaho, Inc.

P.0. Box 1625

Idaho, Falls ID 83401

Director

Nuclear Safety \& Ana1. Development

Electric Power Research Institute

$3412 \mathrm{Hill}$ view Avenue

Palo Alto, CA 94304

Manager

Reactor Systems Safety

Development

General ETectric Company

175 Curtner Avenue

San Jose, CA 95125 
No. of

Copies

OFFSITE

Joel R. Buchanan

Assistant Director

Nuclear Safety Information Center

P. 0. BoX Y

Oad Ridge, TN 37830

$40 \quad$ NRC Safety Research Exchange

ORNL Laboratory Records

Nuclear Safety Information Ctr.

P.0. Box X, Bldg. 4500

Oak Ridge, TN 37830

Executive Director

ACRS

US NRC

HT016

Washington, D.C. 20555

Robert Ritzmann

Science Applications, Inc.

2680 Hanover Street

Palo Alto, CA 94303

40 Adm. Dist. Serv. Branch - Shelf

US NRC

Washington, D.C. 20555

Engineering Manager

Nuclear Fuel Division

Westinghouse Electric Corp.

P.0. Box 355

Pittsburgh, PA 15230

Branch Chief

NRC Core Performance Branch

US NRC

P-1114

Washington, D.C. 20555
No. of

Copies

ONSITE

48

Pacific Northwest Laboratory

W. 3. Bailey

J. L. Bates

J. 0. Barner

E. R. Bradley

D. W. Brite

E. L. Courtright

M. E. Cunningham

J. L. Daniel

N. C. Davis

M. D. Freshley

J. F. Garnier

R. L. Goodman

R. J. Guenther

C. R. Hann (10)

P. E. Hart

P. L. Hendrick

K. A. Hsieh

F. M. Kustas

D. D. Lanning

R. K. Marsha 11

R. P. Marshali

G. Mellinger

C. L. Mohr

C. Neally

T. G. Odekird

F. E. Panisko

P. J. Pankaskie

R. E. Schreiber

S. R. Wagoner

G. D. White

R. E. Williford

C. L. Wilson

Tech. Info. Files (5)

Publishing Coordination (2) 